University of Louisville

ThinkIR: The University of Louisville's Institutional Repository

Electronic Theses and Dissertations

$12-2014$

\title{
Evaluating the effects of aroclor 1260 in non-alcoholic fatty liver disease : the role of xenobiotic receptors.
}

\author{
Banrida Wahlang \\ University of Louisville
}

Follow this and additional works at: https://ir.library.louisville.edu/etd

Part of the Cell and Developmental Biology Commons, and the Pharmacology, Toxicology and Environmental Health Commons

\section{Recommended Citation}

Wahlang, Banrida, "Evaluating the effects of aroclor 1260 in non-alcoholic fatty liver disease : the role of xenobiotic receptors." (2014). Electronic Theses and Dissertations. Paper 1733.

https://doi.org/10.18297/etd/1733

This Doctoral Dissertation is brought to you for free and open access by ThinkIR: The University of Louisville's Institutional Repository. It has been accepted for inclusion in Electronic Theses and Dissertations by an authorized administrator of ThinkIR: The University of Louisville's Institutional Repository. This title appears here courtesy of the author, who has retained all other copyrights. For more information, please contact thinkir@louisville.edu. 
EVALUATING THE EFFECTS OF AROCLOR 1260 IN NON-ALCOHOLIC FATTY LIVER DISEASE: THE ROLE OF XENOBIOTIC RECEPTORS

\author{
By
}

\title{
Banrida Wahlang
}

\author{
A Dissertation \\ Submitted to the Faculty of the \\ School of Medicine of the University of Louisville \\ In Partial Fulfillment of the Requirements \\ For the Degree of
}

Doctor of Philosophy

Department of Pharmacology and Toxicology

University of Louisville

Louisville, KY

December 2014 

EVALUATING THE EFFECTS OF AROCLOR 1260 IN NON-ALCOHOLIC FATTY LIVER DISEASE: THE ROLE OF XENOBIOTIC RECEPTORS

\author{
By \\ Banrida Wahlang \\ Dissertation Approved on \\ November 17, 2014 \\ By the following Dissertation Committee
}

\begin{tabular}{c}
\hline Matthew C. Cave, M.D. \\
\hline Barbara J. Clark, Ph.D. \\
\hline J. Christopher States, Ph.D. \\
\hline Gavin E. Arteel, Ph.D. \\
\hline
\end{tabular}

Russell A. Prough, Ph.D. 


\section{ACKNOWLEDGEMENTS}

I am deeply grateful to my mentor, Dr. Matthew Cave, for his insight, patience and encouragement and Dr. Cameron Falkner, for his continuous support and guidance. They made this dissertation possible. My gratitude also goes to my committee members, Dr. Prough, Dr. Clark, Dr. States and Dr. Arteel for offering their expertise and advice into my project over the years. I am also very grateful to my family and friends for their love and support, always. 


\begin{abstract}
EVALUATING THE EFFECTS OF AROCLOR 1260 IN NON-ALCOHOLIC FATTY LIVER DISEASE: THE ROLE OF XENOBIOTIC RECEPTORS
\end{abstract}

\author{
Banrida Wahlang
}

November 17, 2014

Polychlorinated biphenyls (PCBs) are persistent environmental toxicants, present in $100 \%$ of US adults and dose-dependently associated with nonalcoholic fatty liver disease (NAFLD) in epidemiologic studies. PCBs are predicted to interact with receptors previously implicated in xenobiotic/energy metabolism and NAFLD. These receptors include the aryl hydrocarbon receptor $(\mathrm{AhR})$, pregnane xenobiotic receptor (PXR), constitutive androstane receptor (CAR), peroxisome proliferator-activated receptors (PPARs), liver-X-receptor and farnesoid-X-receptor.

This study evaluated the hepatic effects of the PCB mixture, Aroclor 1260, whose composition mimics human bioaccumulation patterns, in a mouse model of diet-induced obesity. Male C57BI/6J mice were fed a control or $42 \%$ high fat diet (HFD) and exposed to Aroclor 1260 (20 or $200 \mathrm{mg} / \mathrm{kg}$ in corn oil) for 12 weeks. Aroclor 1260 exposure was associated with decreased body fat in HFD- 
fed mice. Aroclor 1260+HFD co-exposed mice demonstrated increased inflammatory foci at both doses while serum cytokines and hepatic expression of IL-6 and TNFa were increased only at $20 \mathrm{mg} / \mathrm{kg}$. Aroclor 1260 induced hepatic Cyp3a11 (PXR target) and Cyp2b10 (CAR target) expression but Cyp2b10 inducibility was diminished with HFD-feeding. Cyp1a2 (AhR target) was induced only at $200 \mathrm{mg} / \mathrm{kg}$.

In $\mathrm{PXR}^{-/-}$and $\mathrm{CAR}^{-/-}$mice, Aroclor 1260 exposure resulted in steatohepatitis with increased basal hepatic TNFa and IL-6 expression. PXR ${ }^{-/-}$ mice had increased \% body fat and liver to body weight ratio regardless of exposure. HOMA-IR decreased in all groups following Aroclor 1260 exposure. $\mathrm{PXR}^{-/-}$mice exposed to Aroclor 1260 showed impaired glucose uptake, increased hepatic gluconeogenic and lipogenic gene expression. The knockout groups demonstrated increased basal mTOR1 activity while Aroclor 1260 exposure increased AMPKa activity. Thus, PXR and CAR participate in hepatic energy metabolism and are protective in Aroclor 1260-induced liver injury.

The study further evaluated Aroclor 1260 and selected congeners as potential ligands for human receptors utilizing HepG2 and COS-1 cell lines; and primary human hepatocytes. The results suggested that Aroclor 1260 is a human AhR, PXR and CAR3 agonist, a mixed agonist/antagonist for CAR2 and an antagonist for human PPARa.

In summary, Aroclor 1260 worsened hepatic inflammation in diet-induced obesity. HFD decreased the protective CAR/PXR activation illustrating the importance of dietary co-exposures in PCB-mediated steatohepatitis. 


\section{TABLE OF CONTENTS}

Page

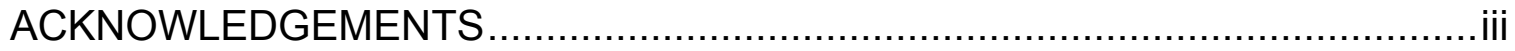

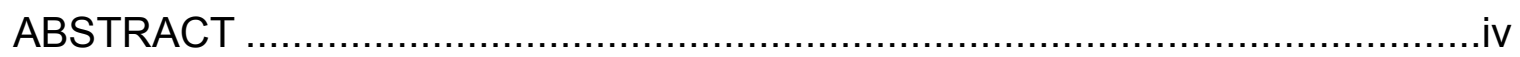

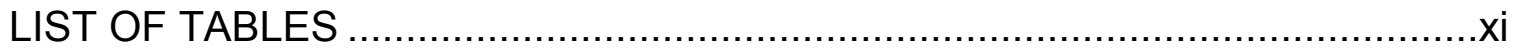

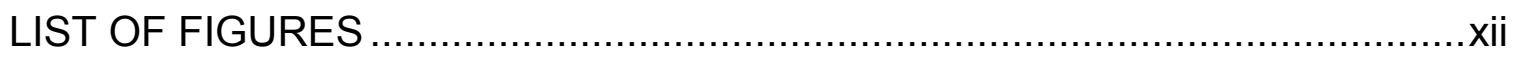

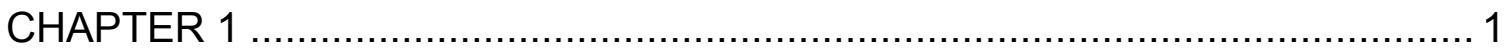

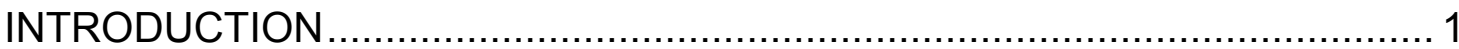

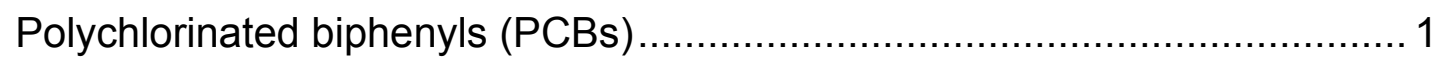

PCBs'structure-activity and toxicity relationship .................................... 2

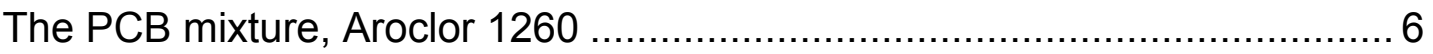

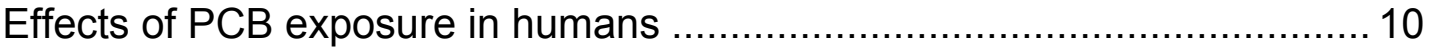

Non-alcoholic fatty liver disease and xenobiotic receptors ........................ 11

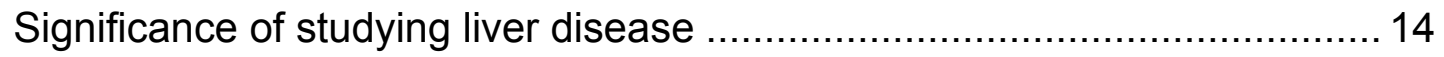

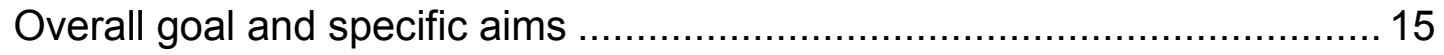

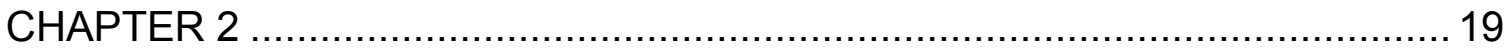

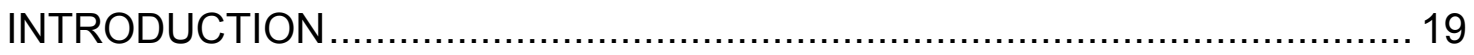

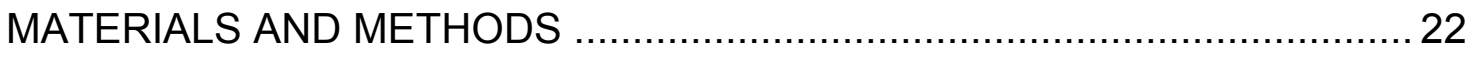


Animals and diets

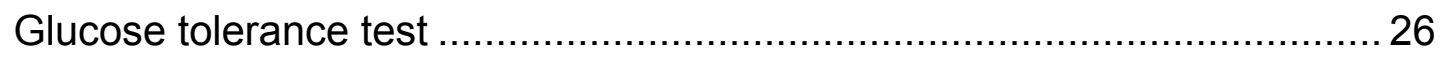

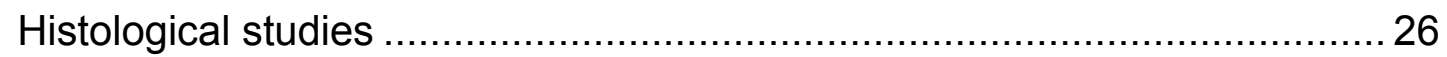

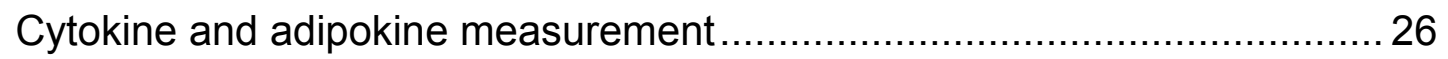

Measurement of hepatic triglyceride and cholesterol content .................... 27

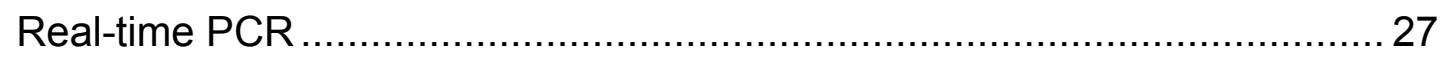

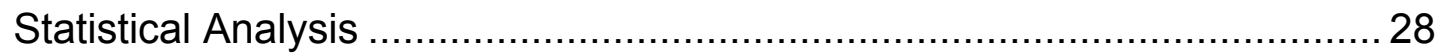

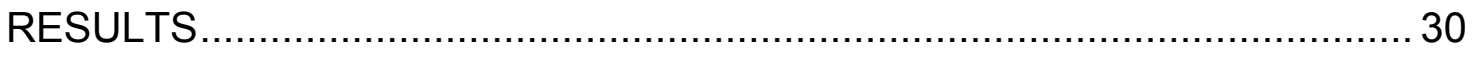

Aroclor 1260 decreased body weight and visceral adiposity in mice fed high

fat diet, but had no effect in mice fed control diet...................................... 30

Aroclor 1260 exposure caused increased liver injury in HFD-fed mice .........34

Effects of Aroclor 1260 exposure on serum adipo-cytokines ..................... 40

Effects of Aroclor 1260 on insulin resistance, glucose regulation, and serum

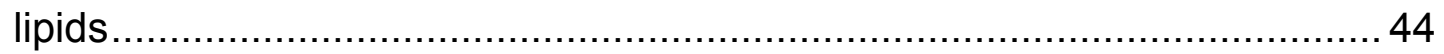

Aroclor 1260 exposure modulated hepatic fat metabolism ........................ 49

Hepatic expression of TLR-4 target genes .............................................. 55

Aroclor 1260 induced hepatic CAR, PXR and AhR target genes ................58

DISCUSSION

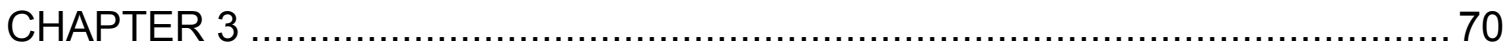

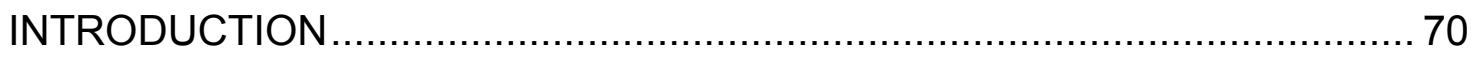




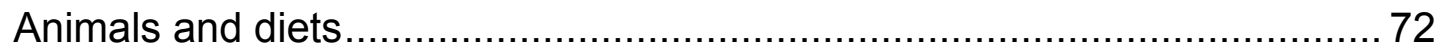

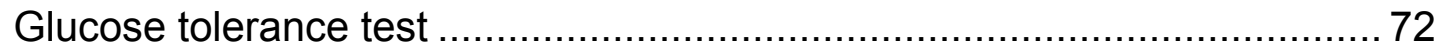

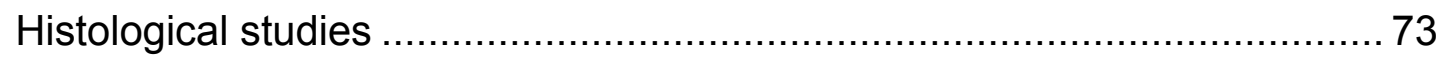

Cytokine and adipokine measurement ................................................ 73

Measurement of hepatic triglyceride and cholesterol content ..................... 73

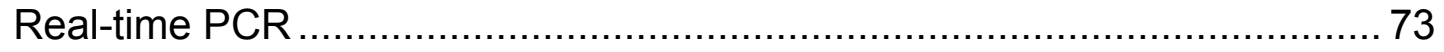

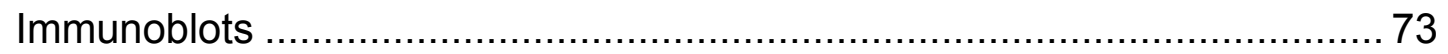

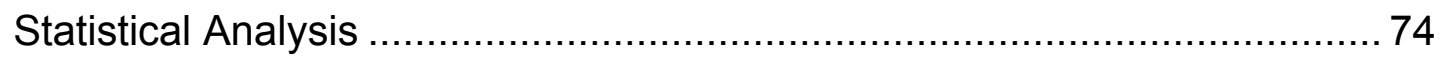

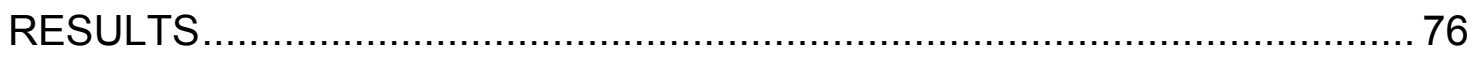

Effects of CAR and PXR on bodyweight and adiposity ............................ 76

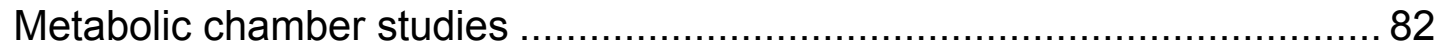

The role of CAR and PXR in Aroclor 1260-induced liver injury and steatosis

CAR and PXR are protective against Aroclor 1260-induced hepatic and

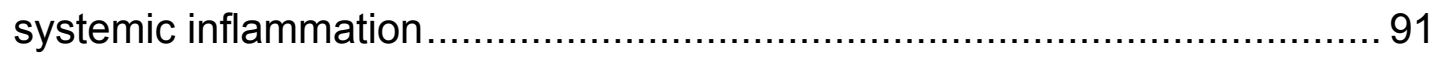

Aroclor 1260, CAR and PXR regulation in glucose metabolism and insulin

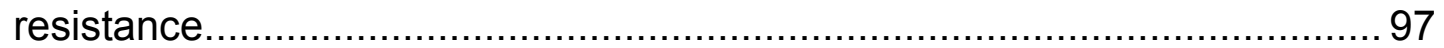

CAR and PXR ablation altered serum adipokine levels .......................... 103 Aroclor 1260, CAR and PXR modulated hepatic lipid metabolism and energy

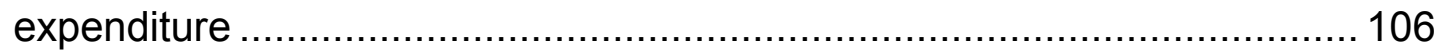


Effects of CAR/PXR on peroxisome proliferator-activated receptor alpha target genes

CAR and PXR target gene induction................................................. 119

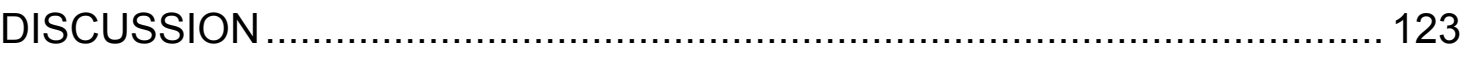

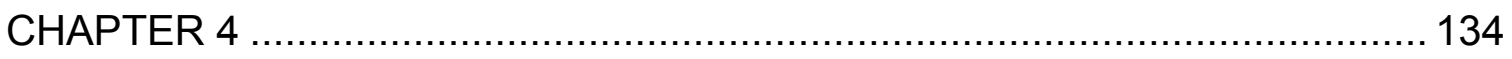

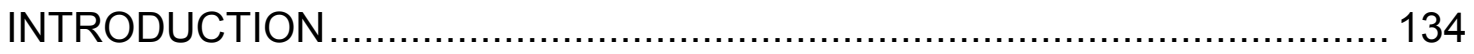

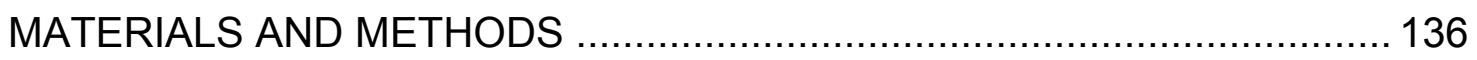

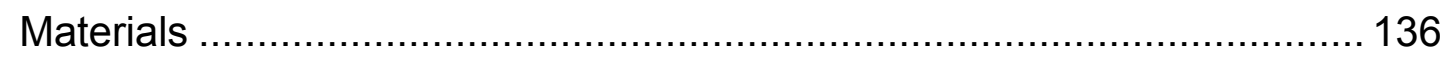

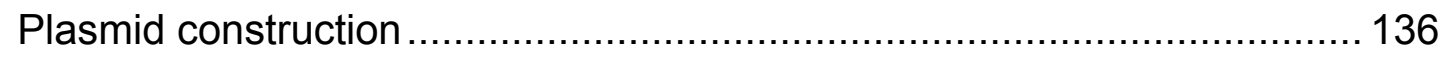

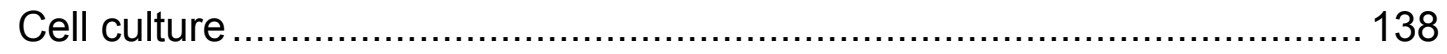

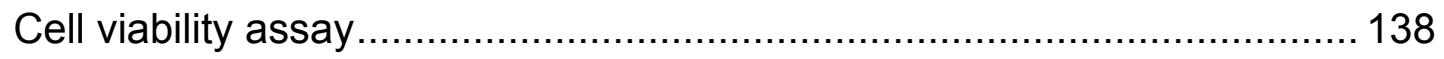

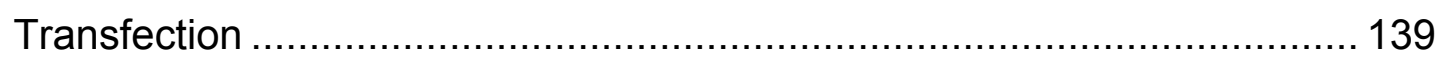

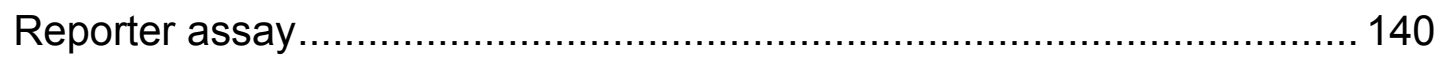

Validation of receptor activation in primary human hepatocytes ............... 141

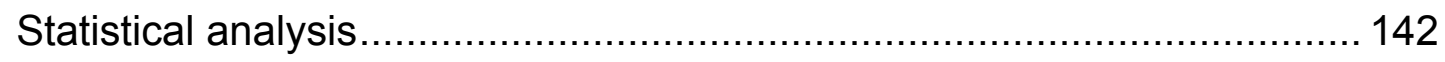

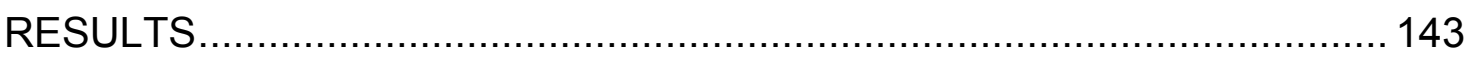

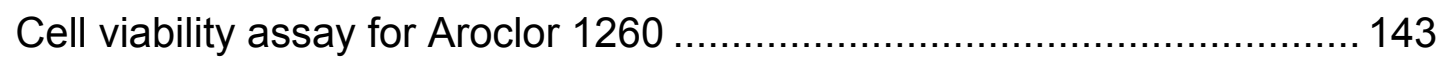

Aroclor 1260 activation of the human aryl hydrocarbon receptor .............. 145

Aroclor 1260 activation of the human pregnane xenobiotic receptor ......... 149

Aroclor 1260 activation of the human constitutive androstane receptor..... 153 
Effect of Aroclor 1260 on the human liver-X-receptor alpha

Effect of Aroclor 1260 on the human farnesoid-X-receptor

Effect of Aroclor 1260 on the human peroxisome-proliferator activated

receptors 165

Aroclor 1260 exposure and gene expression in human hepatocytes 170

DISCUSSION 173

CHAPTER 5 180

OVERALL SUMMARY 180

Overall goal and specific aims 180

Major findings of this dissertation 181

Strengths of this dissertation 186

Limitations of this dissertation 187

Future directions 188

Conclusion 191

REFERENCES 194

APPENDIX 219 CURRICULUM VITAE 228 


\section{LIST OF TABLES}

Page

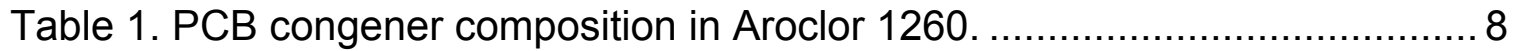

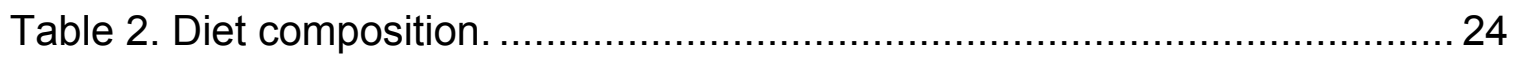

Table 3. Serum levels of cholesterol, high density lipoproteins, low density

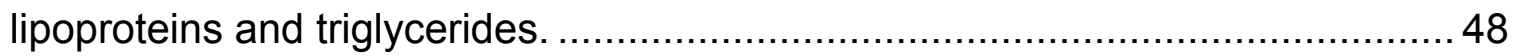




\section{LIST OF FIGURES}

Figure 1.1. Chemical structure of PCBs.

Figure 1.2. Congener composition in human fat, Aroclor 1260 and manufactured

PCBs.

Figure 2.1. Experimental design and timeline.

Figure 2.2. Effects of Aroclor 1260 exposure on body weight and visceral

adiposity.

Figure 2.3. Aroclor 1260 exposure caused steatohepatitis in HFD-fed mice but

had no effect on fibrotic markers.

Figure 2.4. Effects of Aroclor 1260 exposure on hepatic cholesterol and

triglycerides.

Figure 2. 5. Effects of Aroclor 1260 on serum adipo-cytokines.

Figure 2.6.HFD increased insulin resistance, and this was unaffected by Aroclor 1260 co-exposure. 47

Figure 2.7. Effects of Aroclor 1260 exposure on genes involved in lipid

metabolism.

Figure 2.8. Effects of Aroclor 1260 exposure on genes involved in lipid oxidation.

Figure 2.9. Effects of Aroclor 1260 exposure on TLR-4 target genes 57 
Figure 2.10. Aroclor 1260 exposure altered hepatic expression of CAR, PXR and AhR target genes. 61

Figure 3.1. Effects of Aroclor 1260 exposure on body weight and visceral adiposity in $\mathrm{CAR}^{-/-}$and $\mathrm{PXR}^{-/-}$mice. 79

Figure 3.2. Effects of Aroclor 1260 exposure on the adipocyte size and food consumption in $\mathrm{CAR}^{-/-}$and $\mathrm{PXR}^{-l_{-}}$mice.

Figure 3.3. Assessment of respiration exchange rate and energy expenditure utilizing metabolic cages

Figure 3.4. Assessment of movement, food and drink consumption utilizing metabolic cages

Figure 3. 5. Aroclor 1260 exposure caused steatohepatitis in WT, $\mathrm{CAR}^{-{ }^{--}}$and $\mathrm{PXR}^{-/-}$mice

Figure 3.6. Effects of Aroclor 1260 exposure on the TLR-4 target genes in CAR ${ }^{-1-}$ and $\mathrm{PXR}^{-/-}$mice.

Figure 3.7. Effects of Aroclor 1260 exposure on serum cytokines in $\mathrm{CAR}^{-/-}$and $\mathrm{PXR}^{-/-}$mice.

Figure 3.8. Effects of Aroclor 1260, CAR and PXR in glucose metabolism and insulin resistance. 100 Figure 3.9. Effects of Aroclor 1260, CAR and PXR on genes involved in glucose metabolism 102

Figure 3.10. CAR/PXR knockout mice demonstrated altered serum adipokine levels. 
Figure 3.11. Effects of Aroclor 1260, CAR and PXR on hepatic expression of lipogenic and lipolytic genes.

Figure 3.12. Effects of Aroclor 1260, CAR and PXR on hepatic SREBP-1C expression and protein levels.

Figure 3.13. Effects of Aroclor 1260, CAR and PXR on mTOR1 and AMPKa

protein levels. 115

Figure 3.14. Effects of Aroclor 1260 exposure and CAR/PXR ablation on hepatic PPARa expression and its target genes. 118 Figure 3.15. Aroclor 1260 exposure altered hepatic expression of CAR and PXR target genes 122

Figure 3. 16. Effects of CAR and PXR on hepatic energy metabolism. 133

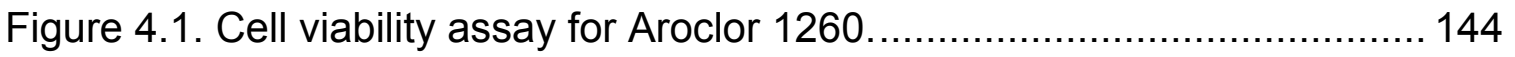

Figure 4.2. Aroclor 1260 activation of the human AhR. .............................. 148

Figure 4.3. Aroclor 1260 activation of the human PXR ................................ 152

Figure 4.4. Aroclor 1260 activation of the human CAR2 transcript. ................. 156

Figure 4.5. Aroclor 1260 activation of the human CAR variant transcripts in COS1 cells 158

Figure 4.6. Aroclor 1260 activation of the human LXRa. 161

Figure 4.7. Aroclor 1260 activation of the human FXR............................... 164

Figure 4.8. Aroclor 1260 activation of the human PPARa. ............................ 167

Figure 4.9. Aroclor 1260 activation of the human PPARy............................. 169

Figure 4.10. Effects of Aroclor 1260 on target genes in primary hepatocytes. . 172 
Figure 5.1. Environmentally-relevant PCB-diet interactions mediate the transition

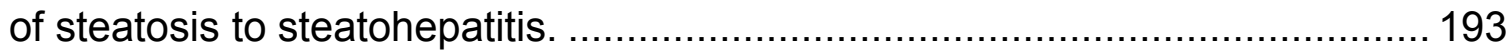




\section{CHAPTER 1}

\section{INTRODUCTION}

\section{Polychlorinated biphenyls (PCBs)}

Polychlorinated biphenyls (PCBs) are polyhalogenated aromatic hydrocarbons consisting of up to 10 chlorine atoms attached to a biphenyl group. Depending on the number of chlorine atom substituents, there are 209 individual PCB congeners that are theoretically possible. PCBs were commercially produced during the 1930s-1970s and were used as dielectric and heat transfer fluids in electric transformers and capacitors, wax extenders, organic diluents, plasticizers, adhesives, flame retardants and as a source of chlorine content (1, 2). At least 1.3 million tons of PCBs, comprising about 130 identified individual congeners, were manufactured worldwide prior to their banning (3). PCB production was prohibited by the United States Congress in 1979 under the Toxic Substances Control Act and PCB production was banned internationally at the Stockholm Convention on Persistent Organic Pollutants in 2001 (4).

Although PCB production has been banned for over 30 years, their high thermodynamic stability make them resistant to chemical and enzymatic degradation in the environment (5) and hence, PCBs, belong to the category of "persistent organic pollutants" (POPs). Moreover, PCBs persist in our ecosystem

globally due to PCB seepage into the environment, accidental spills and improper 
disposal and are found in soil, atmospheric air, lakes, rivers, aquatic wildlife and mammals (6-10). In humans, PCBs, primarily the highly chlorinated congeners that are resistant to metabolism, bio-accumulate and are detected in the adipose tissue, liver, serum and breast milk $(11,12)$.

Currently, PCB exposure in humans is thought to occur primarily through ingestion of PCB-contaminated food $(13,14)$ and to a lesser extent, through inhalation of PCB-contaminated air $(15,16)$. In fact, PCBs continue to be present in the food supply and the intake in the American diet is estimated to be approximately $30 \mathrm{ng} /$ day based on a study from a Dallas supermarket (17). Furthermore, PCBs are the $5^{\text {th }}$ most hazardous substances on the Agency for Toxic Substances and Disease Registry (ATSDR) 2013 substance priority list. The National Health and Nutrition Examination Study (NHANES) indicated that $100 \%$ of adult NHANES participants had detectable circulating PCB levels and PCB $153\left(2,2^{\prime}, 4,4^{\prime}, 5,5^{\prime}\right.$-hexachlorobiphenyl) had the highest median serum concentration in humans amongst all PCB congeners (18). Therefore, although PCB production has stopped, these compounds are ubiquitous in the environment and their resistance to degradation increases the risk of exposure in humans.

\section{PCBs'structure-activity and toxicity relationship}

The PCB's structure more or less determines the compound's activity and toxicity. PCBs can be classified as either "planar" or "non-coplanar" depending on the number and nature of the chlorine substituents in the two phenyl rings, (Fig. 1.1). Planar PCBs have chlorine substitutions in either the meta- or para- 
positions but zero or one substituent in the ortho- positions (19). Non-coplanar PCBs have ortho- substituted chlorine atoms, apart from meta- and/or parasubstitutions (19).

From a mechanistic standpoint, a PCB's structure determines its ability to interact with various receptors (20). PCB studies have demonstrated that planar PCBs such as PCB 77 and PCB 126 interact with the aryl hydrocarbon receptor (AhR) similar to the classic AhR ligand, 2, 3, 7, 8-tetrachlorodibenzo-p-dioxin (TCDD) and hence are also known as "dioxin-like" PCBs $(21,22)$. On the other hand, some non-coplanar PCBs such as PCB 153 and PCB 196 are referred to as "phenobarbital-like" PCBs suggesting their activation of the constitutive androstane receptor (CAR) (22-24). Moreover, a theoretical structure-activity relationship study predicted that non-coplanar PCBs can interact with the pregnane xenobiotic receptor (PXR), estrogen receptor, androgen receptor, and thyroid receptor as well (25).

Historically, PCB studies have focused on hepatocarcinogenesis and other cancer-related endpoints and attributed the mechanism of toxicity to AhR activation $(26,27)$. However, by mass, dioxin-like PCBs are a relatively minor component of the total PCB burden in human serum (18). Moreover, PCBs do not appear to be as carcinogenic in man as they are in rodents (eg: PCB 126), and this may be due to differences in the AhR structure and in the battery of target genes between species (28). In contrast to PCB 126, PCB 153 is an orthosubstituted, non-coplanar PCB. PCB 153 has been studied extensively by the National Toxicology Program (NTP) in female Harlan Sprague-Dawley (SD) rats, 
and the mode of action has been attributed to the compound's interaction with CAR (23). The studies also demonstrated PCB-induced hepatotoxicity.

Apart from the structure, a PCB congener's molecular weight which corresponds to the degree of chlorination also dictates the type of toxicity. PCB's metabolism is defined by the number of chlorine atoms present. Low molecular weight PCBs (mono-, di-, tri- or tetra-chlorinated) are hydroxylated by cytochrome P450 enzymes (29). The metabolized PCBs, also known as biotransformed congeners, can form DNA adducts or bind to proteins to exert their toxic effects. High molecular weight PCBs, on the other hand, are resistant to metabolism, hence they are known as persistent congeners. Some of these PCBs' half-life is $>15$ years (30). These lipid-soluble PCBs, including the pentachlorinated PCB 126 and hexa-chlorinated PCB 153, bio-accumulate primarily in the adipose tissue and have the ability to interact with receptors (1).

Therefore, depending on the molecular weight of the congener and the molecular structure, PCBs have a tendency to induce carcinogenesis by forming adducts and through AhR activation. PCB exposure can also cause other complications, such as endocrine disruption through other PCB-receptor interactions. Additionally, apart from PCB-receptor interactions, nutrient-toxicant interactions have also been reported to play a role in health disorders such as diet-induced obesity/metabolic syndrome (31) and non-alcoholic fatty liver disease (NAFLD) (32). 


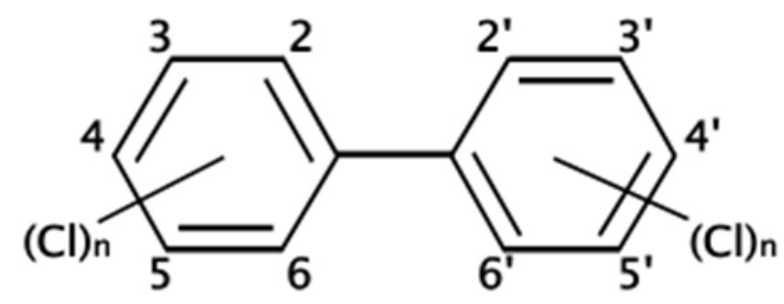

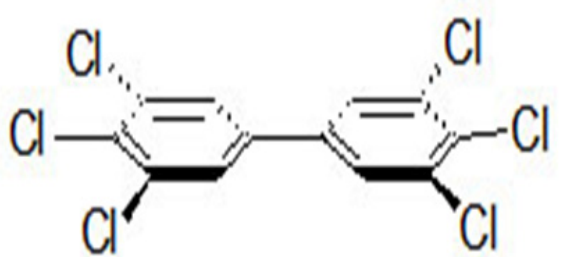

Coplanar<smiles>Clc1ccc(-c2cc(Cl)c(Cl)c(Cl)c2)cc1Cl</smiles>

PCB 126

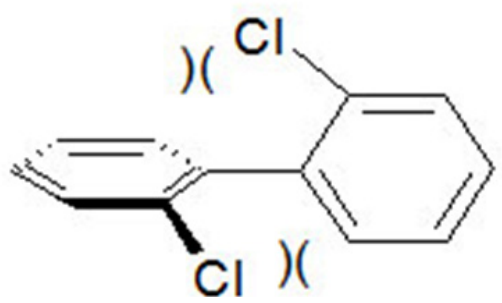

Non-coplanar<smiles>Clc1cc(Cl)c(-c2cc(Cl)c(Cl)cc2Cl)cc1Cl</smiles>

PCB 153

Figure 1.1. Chemical structure of PCBs. 


\section{The PCB mixture, Aroclor 1260}

PCBs were commercially marketed and used as mixtures rather than individual congeners. Monsanto, a well-known PCB manufacturer in North America, produced PCB mixtures under the brand name "Aroclor" at its manufacturing plant located in Anniston, Alabama. Incidences of high-level environmental contamination during PCB production resulted in increased PCB body burden in the Anniston residents $(33,34)$. PCB production was global with

PCB mixtures being manufactured worldwide under different brand names including Clophens (Germany), Phenoclors and Pyralenes (France), Fenclors (Italy), Fenochlors (Spain), Kanechlors (Japan) and Sovol (former USSR) (2). Aroclor 1260 , a commercial PCB mixture, contained $60 \%$ chlorine by weight. It was one of the first generation PCB mixtures produced by Monsanto during the early stages of PCB production. Aroclor 1260 was later replaced by other Aroclors such as Aroclor 1254, Aroclor 1248 and Aroclor 1242 that had lower chlorine content by weight (2). Aroclor 1260 is heavily chlorinated and contains a limited amount of coplanar congeners ( 1\%) (19). It has predominantly non-coplanar and di-ortho substituted PCBs that have either 5, 6, 7, or 8 chlorines (Table 1). As PCBs with low molecular weight are often metabolized and eliminated, PCBs that bio-accumulate in humans are typically the highly chlorinated congeners with penta-, hexa-, hepta-, and octa substituents $(35,36)$. Thus, for our PCB studies, we selected the PCB mixture Aroclor 1260 because its composition best mimics the PCB bioaccumulation 
profile found in human adipose tissue, rather than reflecting the actual production volume of PCBs (Fig. 1.2). 


\begin{tabular}{|c|c|c|c|}
\hline $\begin{array}{c}\text { PCB } \\
\text { congener }\end{array}$ & No. of chlorine & Ortho substitution & $\%$ \\
\hline 180 & 7 & (Non-coplanar, di-ortho) & 11.38 \\
\hline 153 & 6 & (Non-coplanar, di-ortho) & 9.39 \\
\hline 149 & 6 & (Non-coplanar, tri-ortho) & 8.75 \\
\hline 138 & 6 & (Non-coplanar, di-ortho) & 6.54 \\
\hline 187 & 7 & (Non-coplanar, tri-ortho) & 5.40 \\
\hline 174 & 7 & (Non-coplanar, tri-ortho) & 4.96 \\
\hline 170 & 7 & (Non-coplanar, di-ortho) & 4.11 \\
\hline 101 & 5 & (Non-coplanar, di-ortho) & 3.13 \\
\hline 151 & 6 & (Non-coplanar, tri-ortho) & 3.04 \\
\hline 132 & 6 & (Non-coplanar, tri-ortho) & 2.90 \\
\hline 141 & 6 & (Non-coplanar, di-ortho) & 2.62 \\
\hline 177 & 7 & (Non-coplanar, tri-ortho) & 2.57 \\
\hline 95 & 5 & (Non-coplanar, tri-ortho) & 2.45 \\
\hline 163 & 6 & (Non-coplanar, di-ortho) & 2.42 \\
\hline 183 & 7 & (Non-coplanar, tri-ortho) & 2.41 \\
\hline 194 & 8 & (Non-coplanar, di-ortho) & 2.07 \\
\hline 179 & 7 & (Non-coplanar, tetra-ortho) & 2.03 \\
\hline 136 & 6 & (Non-coplanar, tetra-ortho) & 1.46 \\
\hline 203 & 8 & (Non-coplanar, tri-ortho) & 1.40 \\
\hline 110 & 5 & (Non-coplanar, di-ortho) & 1.33 \\
\hline 146 & 6 & (Non-coplanar, di-ortho) & 1.15 \\
\hline 171 & 7 & (Non-coplanar, tri-ortho) & 1.11 \\
\hline 196 & 8 & (Non-coplanar, tri-ortho) & 1.09 \\
\hline 135 & 6 & (Non-coplanar, tri-ortho) & 1.08 \\
\hline
\end{tabular}

\section{Table 1. PCB congener composition in Aroclor 1260.}

Selected PCB congeners present in Aroclor 1260 ( $\geq 1 \%$ of total composition). Table adapted from www.atsdr.cdc.gov/toxprofiles/tp17-c4.pdf. 


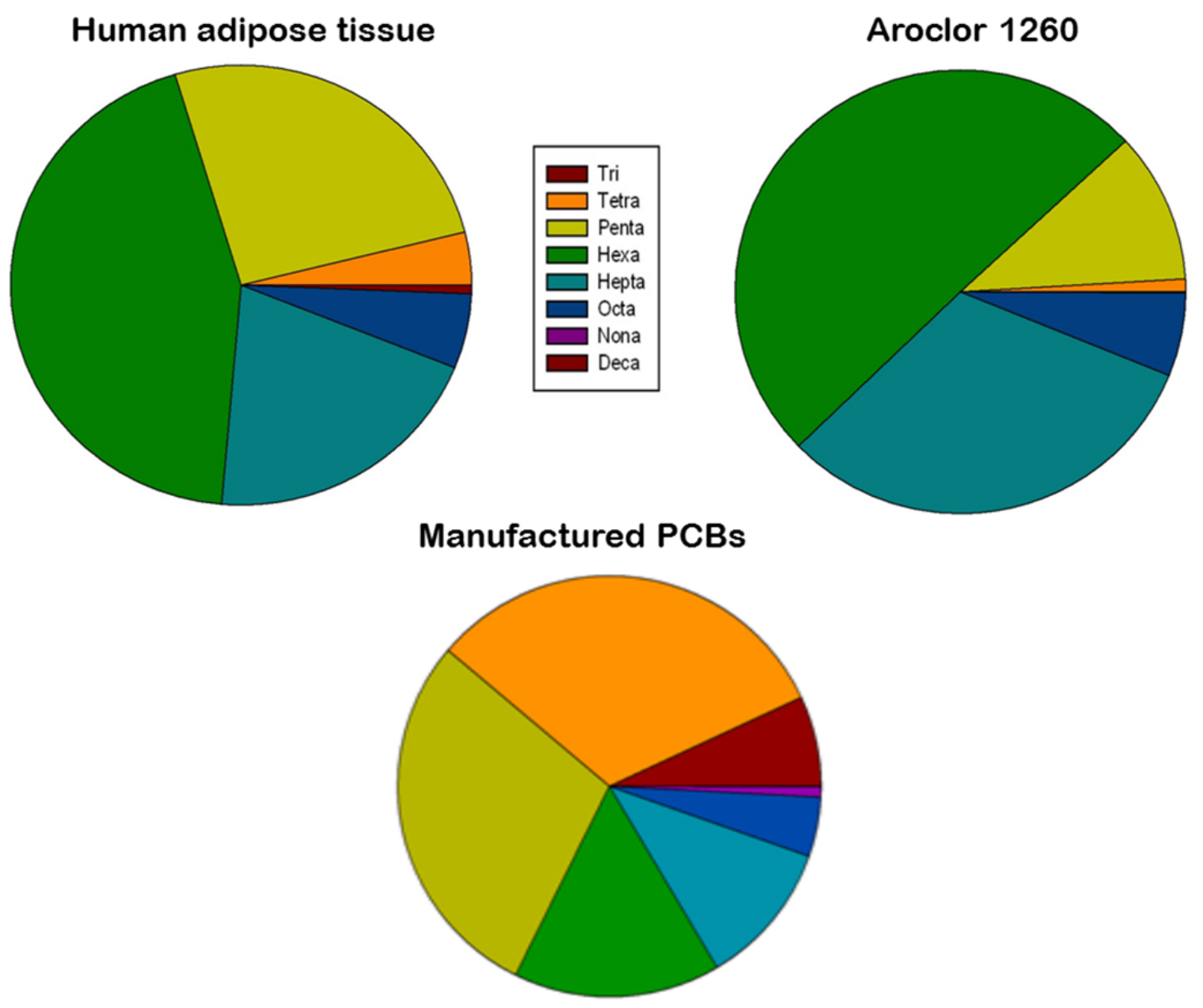

Figure 1.2. Congener composition in human fat, Aroclor 1260 and manufactured PCBs.

Pie charts depicting the relative abundance of PCB congeners in human adipose tissue, Aroclor 1260 and manufactured PCBs. 


\section{Effects of PCB exposure in humans}

Historically, PCB toxicity has been linked to cancer, endocrine disruption and impaired cognitive development (37-40), but recent epidemiologic studies have shown that chronic exposure to these environmental pollutants can result in metabolic disorders associated with fatty liver disease, including obesity, insulin resistance/diabetes and the metabolic syndrome $(18,34,41-44)$. Moreover, other complications associated with metabolic disorders such as hypertension and cardiovascular disorders have also been associated with PCB exposure $(45,46)$. Furthermore, a follow-up of subjects in the "Yu-cheng" incident in Taiwan demonstrated that the mortality rate due to cirrhosis was 2.7 -fold higher than expected, although pathologic confirmation was not provided (47). The "Yucheng" incident was one of the two known major human PCB intoxication episodes, where the victims ingested cooking oil that was highly contaminated with PCBs. Additionally; rodent studies have correlated PCB exposures with NAFLD, obesity and the metabolic syndrome $(48,49)$.

Our laboratory group recently identified advanced steatohepatitis associated with insulin resistance and increased pro-inflammatory cytokines in non-obese chemical workers who were subjected to high-level industrial chemical exposures (50). The term "toxicant-associated steatohepatitis" (TASH) was then coined to describe this condition (51). Our initial work focused on TASH in non-obese vinyl chloride workers; in our recent work however, our laboratory group identified suspected NAFLD and TASH in the NHANES participants with low-level environmental exposures to POPs, including 20 PCBs (18). 
Epidemiologic studies have reported a positive association between adipose tissue concentrations of PCBs and type 2 diabetes (52). High serum PCB levels were also associated with elevated serum triglycerides and cholesterol which are major risk factors for cardiovascular diseases. Exposure to PCB mixtures has also been associated with elevated liver enzymes in plasma and hepatomegaly $(18,53)$. Clearly, environmental exposure to POPs such as PCBs is a major health concern, with emerging studies reporting positive associations between PCB body burden and suspected liver disease. However, studies involving the non-carcinogenic effects of PCB exposure on the liver are scarce and therefore, more research is required to evaluate the role of PCB exposure in liver disease, particularly NAFLD.

\section{Non-alcoholic fatty liver disease and xenobiotic receptors}

NAFLD represents a pathological spectrum of diseases ranging from lipid accumulation in the hepatocytes (steatosis) to the development of superimposed inflammation, leading to non-alcoholic steatohepatitis (NASH) and ultimately fibrosis and cirrhosis. NAFLD resembles alcoholic fatty liver disease, although it occurs in subjects who do not consume excessive amounts of alcohol. The pathogenesis of NAFLD has been reviewed, and key mechanisms included altered adipo-cytokines with low adiponectin and high leptin levels $(32,54)$ and elevation of pro-inflammatory cytokines such as TNFa and IL-6 (55). NAFLD gives rise to hepatic insulin resistance and systemic inflammation, which in turn, exacerbates obesity, diabetes and the metabolic syndrome (56). Because it is often seen with insulin resistance and dyslipidemia, NAFLD is generally regarded 
to be the hepatic manifestation of obesity and the metabolic syndrome. However, NAFLD is also exacerbated by diabetes and is associated with obesity (57), indicating a vicious cycle between NAFLD, diabetes/insulin resistance and obesity.

NAFLD and NASH were traditionally associated with the inappropriate over- or under-activation of nuclear receptors involved in endobiotic metabolism. These receptors include the liver-X-receptor ( $L X R)$, farnesoid-X-receptor (FXR) and peroxisome proliferator-activated receptors (PPARs) which regulate cholesterol, bile acid and lipid metabolism respectively $(58,59)$. Recent studies have implicated the role of hepatic receptors involved in xenobiotic detoxification, including PXR, CAR and AhR in NAFLD/NASH. Although these receptors were initially thought to be involved only with detoxification and xenobiotic metabolism, over-activation or antagonism of these receptors may lead to metabolic diseased states such as steatosis and obesity (60-62). Notably, PCBs have been demonstrated to interact with AhR, CAR and PXR $(22-24,26,27)$. Therefore, it is pertinent to study the activation of these xenobiotic receptors by PCBs and their contribution to NAFLD with PCB exposure.

The AhR is a member of the Per Arnt Sim (PAS) domain protein family of transcription factors and regulates a battery of genes involved in xenobiotic detoxification including CYP1A and CYP1B which can, in turn, bio-transform or activate pro-carcinogens to their carcinogenic forms $(63,64)$. Apart from xenobiotic detoxification, the AhR also plays a role in the differentiation of developmental pathways such as hematopoiesis (65) and in regulating 
immunological responses (66). Furthermore, previous work also implicated dioxins and dioxin-like PCBs in animal models of steatohepatitis $(67,68)$.

On the other hand, PXR, also known as the steroid and xenobiotic sensing nuclear receptor (nuclear receptor subfamily 1, group I, member 2, NR1I2) and CAR (nuclear receptor subfamily 1 , group I, member 3 , NR1I2NR1I3) belong to the class of nuclear receptors; upon activation by ligands, PXR and CAR transcriptionally activate numerous target genes that encode enzymes and transporters involved in drug and xenobiotic metabolism (69). However, recent studies have demonstrated these receptors' role in energy homeostasis and these include regulation of lipid and carbohydrate metabolism $(60,70)$. CAR was recently named as an anti-obesity nuclear receptor, since its activation improves insulin sensitivity in a diet-induced obesity mouse model and ameliorates diabetes and fatty liver disease in leptin-deficient mice $(71,72)$. Activating CAR and PXR also induced the anti-lipogenic protein, Insig (73). Furthermore, PXR and CAR activation also suppressed gluconeogenesis by decreasing gluconeogenic gene expression including phosphoenol-pyruvate carboxykinase and glucose-6-phosphatase (74). The reported role of PXR in obesity is controversial with some studies demonstrating obesity-protecting effects of PXR activation (75) and others illustrating that ablating PXR alleviated diet-induced obesity $(76,77)$. CAR and PXR exert these effects through interactions with other transcription factors and receptors including direct protein sequestration. Activated nuclear receptors bind to the respective DNA-response elements in the promoter regions of the target genes using their conserved DNA-binding domains 
and promote gene expression (78). These response elements are made up of hexameric sequences that can be arranged in different configurations to give direct repeats (DR) or inverted repeats. CAR and PXR can bind to similar DNAresponse elements in the promoter region, increasing the probability of crosstalk. Therefore, CAR and PXR activation can result in crosstalk with other receptors and possibly activation of genes that are not necessarily CAR or PXR targets.

\section{Significance of studying liver disease}

NAFLD affects up to $46 \%$ of the US population and approximately $20 \%$ of the population worldwide and it is the most prevalent liver disease in North America (79-81). It is more common in men than women until the age of 60 when the prevalence matches and the lower rate of NAFLD in women is attributed to the protective nature of estrogen in fatty liver disease. The prevalence of NAFLD is still growing and it is higher in the Hispanic population, which can be attributed to high rates of obesity and type 2 diabetes in this population (82). NAFLD is not an independent entity by itself but it is closely related to obesity, insulin resistance and the metabolic syndrome. The association between NAFLD and obesity poses a great concern to human health because obesity has been identified as a leading "preventable cause of death" by the World Health Organization (WHO), and many health authorities view it as one of the most serious health problems of the $21^{\text {st }}$ century. The annual cost of obesity treatment in the US alone was recently estimated at $\$ 147$ billion (83). According to the data from NHANES $2007-2008,33.8 \%$ of US adults are obese (defined by body mass index, $\mathrm{BMI} \geq 30$ ) with another $34.2 \%$ being overweight (defined by $\mathrm{BMI} \geq$ 
25). Alarmingly, obesity is not restricted to the adult population; $15 \%$ of children and adolescents in the US are obese (84). Overweight/obesity is one of the defining features of the metabolic syndrome, a metabolic disorder which is also characterized by insulin resistance, hypertension, and dyslipidemia. The prevalence of metabolic syndrome in US adults was recently estimated at $34.3 \%$. The obesity epidemic is strongly associated with increased type 2 diabetes which is the late stage of insulin resistance. The National Diabetes Fact Sheet obtained from the Center for Disease Control and Prevention (CDC, 2011) reports that 25.8 million Americans have diabetes with another 79 million being insulin resistant. Obesity, insulin resistance, and metabolic syndrome can eventually lead to target organ damage contributing to cardiovascular disease, chronic kidney disease, and cancer (85-87). Therefore, taking the above mentioned statistics into consideration, studying the causal factors of NAFLD is relevant to public health.

\section{Overall goal and specific aims}

The pathogenesis of NAFLD, obesity, insulin resistance, and the metabolic syndrome is complex. NAFLD and obesity were linked to high caloric intake and over-nutrition, physical inactivity, genetic background, and certain medications' side effects. Interestingly, inflamed and fatty liver conditions are not confined to obese individuals only, but other factors can predispose a person to steatohepatitis. It is possible that primary factors such as consumption of hypercaloric foods can lead to steatosis in normal individuals, thus acting as a 'first hit'. Emerging studies have demonstrated that other factors may play a role in the 
genesis and development of NAFLD or may act as a 'second hit' in the progression of hepatic steatosis to steatohepatitis (88). These factors may include exposure to environmental contaminants including PCBs, bromodichloromethane (BDCM) and organochlorine pesticides, such as, dieldrin and dichlorodiphenyltrichloroethane (DDT) $(41,89)$. Our laboratory group recently identified industrial chemicals and environmental pollutants that are associated with toxicant-associated fatty liver disease and toxicant-associated steatohepatitis (TASH) using two federal databases (90). Such compounds have the capacity to act as key players in the development of $\mathrm{NASH}$, obesity and the metabolic syndrome but the number of studies on the effects of environmental pollutants in liver disease is still modest and needs to be investigated.

The overall goal of this dissertation is to evaluate the role of PCB exposure in fatty liver disease and elucidate the potential PCB-receptor interactions that mediate fatty liver disease. The outcomes from this study would benefit our understanding of TASH and the underlying mechanisms caused by toxicant exposure in TASH. The specific aims of the project are as follows:

1A. Develop a mouse model for PCB exposure that simulates human exposure paradigms.

1B. Evaluate the effects of the commercialized PCB mixture, Aroclor 1260 , in obesity and NAFLD.

The congener composition in the PCB mixture, Aroclor 1260, resembles bio-accumulated PCBs present in human adipose tissue (Fig. 1.2) and it was therefore chosen for this study. Prior to initiating the animal experiments 
described in this dissertation, there were no documented studies on animal models using PCB mixtures that simulate bio-accumulated PCBs and at doses relevant to human exposure. Additionally, the effects of Aroclor 1260 in dietinduced steatosis and obesity have never been assessed. The purpose of the studies in Chapter 2 was to develop a mouse model to study the effects of PCB exposure at doses relevant to human exposure. Additionally, the experiments in Chapter 2 were designed to determine if Aroclor 1260 exposure by itself could induce NAFLD or if it worsens NAFLD and obesity caused by high fat diet feeding.

\section{Determine the role of the nuclear receptors, CAR and PXR, in liver} injury caused by Aroclor 1260 and high fat diet co-exposure.

CAR and PXR are involved in drug metabolism but recent studies have demonstrated in the role of these receptors in maintaining energy homeostasis in the body (60-62). Moreover, theoretical structure activity relationship studies predicted that non-coplanar PCBs can interact with CAR and PXR (25). However activation of CAR and PXR by a PCB mixture and the subsequent contribution of these activated receptors to PCB effects and toxicity have not been thoroughly evaluated, especially in terms of obesity and NAFLD/NASH. The experiments described in Chapter 3 examined the role of CAR and PXR in the context of liver injury caused by Aroclor 1260 exposure and high fat diet feeding.

\section{Examine the interactions between Aroclor 1260/selected PCB} congeners and human receptors in the liver 
We hypothesized that PCBs may exert some of their toxic effects, such as NAFLD, by interacting directly with the endobiotic nuclear receptors (LXR, FXR, PPARs) or with xenobiotic receptors (AhR, CAR and PXR). Moreover, the interaction between Aroclor 1260 and these receptors that are implicated in NAFLD have never been tested. The purpose of the studies in Chapter 4 is to evaluate human receptor agonism/antagonism by the PCB mixture, Aroclor 1260, and selected PCB congeners that are highly represented in this mixture. We determined human receptor activation by utilizing transient transfection assays in human hepatoma-derived (HepG2) and primate-derived (COS-1) cell lines. 
CHAPTER 2

\section{EVALUATING THE EFFECTS OF AROCLOR 1260 IN A DIET-INDUCED OBESITY MOUSE MODEL}

\section{INTRODUCTION}

As aforementioned, previous studies on PCBs were focused on carcinogenicity and acute toxicity; however, the effects of chronic low-level exposures to PCBs on other pathologies such as NAFLD, diabetes and obesity are understudied, especially for PCB mixtures. Epidemiological studies have found associations between PCB exposures and metabolic disorders related to NAFLD, including obesity, diabetes/insulin resistance and the metabolic syndrome. In our recent work, we demonstrated that PCB 153 administration was a relevant 'second hit' mechanism that worsened NAFLD/obesity occurring in the context of a high fat diet (HFD) in male C57BI/6 mice (48). However, no individual is exposed to a single PCB congener alone and therefore studying a PCB mixture may better simulate human PCB exposure patterns. Moreover, the composition of PCB congeners in Aroclor 1260 mimics human bioaccumulation patterns (36).

The primary toxicity endpoint of PCB exposure has been an increase in cancer and is thought to be mediated through PCB interaction with transcription factors namely the AhR. Genotoxic carcinogenic mechanisms are activated via 
induction of the AhR and the carcinogenicity of a PCB mixture is often defined as its ability to induce the AhR-dependent gene transcription relative to the potent rodent carcinogen and prototypical AhR ligand, TCDD. In addition, "non genotoxic" carcinogenic mechanisms may also be induced including hepatomegaly by the nuclear receptors, CAR and PXR. Although these receptors are primarily involved in xenobiotic detoxification, recent studies have implicated their role in metabolic diseased states such as steatosis, obesity and insulin resistance (60-62). The role of hepatic receptor-PCB interactions in NASH and the metabolic syndrome is understudied and requires further investigation.

It is therefore pertinent to evaluate the effects of a commercially produced PCB mixture whose composition is relevant to human PCB body burden, in a normal and hyper- caloric state, and investigate nutrient-toxicant interactions that can contribute to liver disease and obesity. We hypothesize that the commercial PCB mixture, Aroclor 1260, like PCB 153, can act as an obesogen and promote/worsen steatosis and diet-induced obesity. In this study, we utilized a mouse model to determine if i) Aroclor 1260 exposures alone are sufficient to cause NAFLD and obesity; ii) Aroclor 1260 worsens diet-induced obesity and NAFLD by exacerbating insulin resistance, adipocytokine dysregulation, alterations in hepatic gene expression, AhR, PXR and CAR activation and other implicated mechanisms; and iii) effects observed with Aroclor 1260 administration are dependent on PCB dose.

The results of the current study established Aroclor 1260 as a "second hit" driving steatosis to steatohepatitis in HFD-fed mice. The results also 
demonstrated that selective hepatic receptor activation by this PCB mixture was dependent on exposure dose and diet, in part, clarifying the mechanism of action of PCBs in fatty liver disease. 


\section{MATERIALS AND METHODS}

\section{Animals and diets}

The animal protocol was approved by the University of Louisville Institutional Animal Care and Use Committee. Male C57BI/6J mice (8 weeks old; The Jackson Laboratory, Bar Harbor, ME, USA) were divided into 6 study groups $(n=10)$ based on diet and Aroclor 1260 exposure in this 12 week study utilizing a $2 \times 3$ design. Mice were fed either a control diet (CD, 10.2\% kCal from fat; TD.06416 Harlan Teklad) or a HFD (42\% kCal from fat; TD.88137 Harlan Teklad). Diet components are described in Table 2. Aroclor 1260 (AccuStandard, CT, USA) was administered in corn oil by oral gavage (vs. corn oil alone) at two doses; a low dose of $20 \mathrm{mg} / \mathrm{kg}$ which was designed to mimic the maximum human PCB exposures seen in the Anniston cohort and a high dose of 200 $\mathrm{mg} / \mathrm{kg}$ which was similar to that used in rodent carcinogenesis studies $(18,22)$. The $20 \mathrm{mg} / \mathrm{kg}$ dose was administered on Week 1 whereas the $200 \mathrm{mg} / \mathrm{kg}$ dose was administered as four individual doses of $50 \mathrm{mg} / \mathrm{kg}$ each on Weeks 1, 3, 5, and 7 to ensure that acute toxicity was minimized (Fig. 2.1). Mice were housed in a temperature- and light controlled-room (12 hour light; 12 hour dark) with food and water ad libitum. A glucose tolerance test was performed at Week 11, and the animals were euthanized (ketamine/ xylazine, $100 / 20 \mathrm{mg} / \mathrm{kg}$ body weight, i.p.) at the end of Week 12. Prior to euthanasia, the animals were analyzed for body fat composition by dual energy X-ray absorptiometry (DEXA) scanning (Lunar PIXImus densitometer, WI, USA). Thus six different treatment groups were evaluated in this fashion: CD+vehicle, CD+Aroclor $1260(20 \mathrm{mg} / \mathrm{kg})$, 
CD+Aroclor 1260 (200 mg/kg), HFD+vehicle, HFD+Aroclor 1260 (20 mg/kg), HFD+Aroclor $1260(200 \mathrm{mg} / \mathrm{kg})$. 


\begin{tabular}{lllll}
\hline & \multicolumn{2}{c}{ Control Diet } & \multicolumn{2}{c}{ High Fat Diet } \\
\cline { 2 - 5 } & \%kCal & Source & \%kCal & Source \\
\hline Protein & 20.1 & Casein & 15.2 & Casein \\
Carbohydrate & 69.8 & $\begin{array}{l}\text { Sucrose/Corn } \\
\text { starch }\end{array}$ & 42.7 & Sucrose/Corn \\
& & starch & Anhydrous \\
Fat & 10.2 & Lard & 42 & Milkfat \\
kCal/g & 3.7 & & 4.5 & \\
\hline
\end{tabular}

Table 2. Diet composition. 


\section{Male C57BL/6 mice ( 8 weeks old, $n=10$ )}

1. Control Diet (CD, $10 \%$ kCal fat)

3. $C D+A r 1260(20 \mathrm{mg} / \mathrm{kg})$

5. CD+Ar $1260(200 \mathrm{mg} / \mathrm{kg})$
2. High Fat Diet (HFD, $42 \%$ kCal fat)

4. HFD+Ar $1260(20 \mathrm{mg} / \mathrm{kg})$

6. $H F D+A r 1260(200 \mathrm{mg} / \mathrm{kg})$

Aroclor 1260 by gavage (20 $\mathrm{mg} / \mathrm{kg}$ in corn oil)

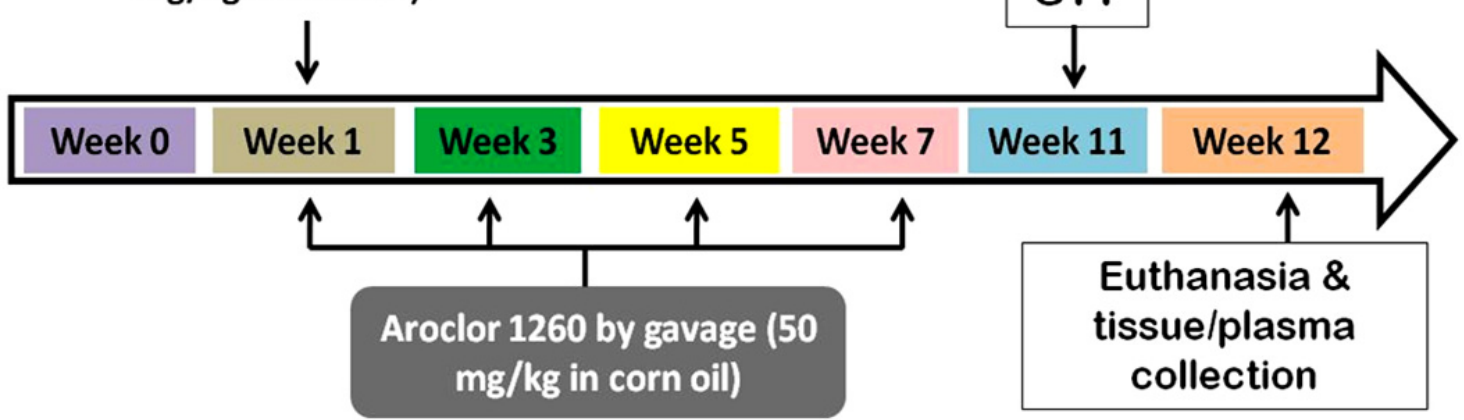

Figure 2.1. Experimental design and timeline 


\section{Glucose tolerance test}

On the day of the test, mice were fasted for $6 \mathrm{~h}$ (7a.m.-1 p.m.), and fasting blood glucose levels were measured with a hand-held glucometer (ACCUCHECK Aviva, Roche, Basel, Switzerland) using 1-2 $\mu \mathrm{L}$ blood via tail snip.

Glucose was then administered ( $1 \mathrm{mg}$ glucose/g body weight, sterile saline, i.p.), and blood glucose was measured at 5, 15, 30, 60, 90 and 120 min post injection. Insulin resistance was calculated by homeostasis model assessment using the formula: homeostasis model assessment of insulin resistance (HOMA$\mathrm{IR})=$ Fasting glucose $(\mathrm{mg} / \mathrm{dL}) \times$ Fasting insulin $(\mu \mathrm{U} / \mathrm{mL}) / 405$.

\section{Histological studies}

Liver and adipose sections were fixed in $10 \%$ neutral buffered formalin and embedded in paraffin for routine histological examination. Tissue sections were stained with hematoxylin-eosin (H\&E) or for chloroacetate esterase activity [CAE, Naphthol AS-D Chloroacetate (Specific Esterase) Kit, Sigma Aldrich, St. Louis, MO, USA] and examined by light microscopy. Photomicrographic images were captured using a high-resolution digital scanner at 10x and $40 \mathrm{x}$ magnification. After H\&E staining, adipocyte size was measured using Image $\mathrm{J}$ software version 1.47 ( $\mathrm{NIH}$, Bethesda, MD, USA).

\section{Cytokine and adipokine measurement}

Plasma cytokine and adipokine levels were measured using the Milliplex Serum Cytokine and Adipokine Kits (Millipore Corp, Billerica, MA, USA) on the Luminex IS 100 system (Luminex Corp, Austin, TX, USA), as per the manufacturer's instructions. Plasma aspartate transaminase (AST) activity, 
alanine transaminase (ALT) activity, low density lipoprotein (LDL), high density lipoprotein (HDL), triglycerides and cholesterol levels were measured with the Piccolo Xpress Chemistry Analyzer using Lipid Panel Plus reagent discs (Abaxis, Union City, CA, USA).

\section{Measurement of hepatic triglyceride and cholesterol content}

Mouse livers were washed in neutral $1 \mathrm{X}$ phosphate buffered saline and pulverized. Hepatic lipids were extracted by an aqueous solution of chloroform and methanol, according to the Bligh and Dyer method (91), dried using nitrogen, and resuspended in $5 \%$ lipid-free bovine serum albumin. Triglycerides and cholesterol were quantified using the Cobas Mira Plus automated chemical analyzer. The reagents employed for the assay were L-Type Triglyceride M (Wako Diagnostics, Richmond, VA, USA) and Infinity Cholesterol Liquid Stable Reagent (Fisher Diagnostics, Middletown, VA, USA) for triglycerides and cholesterol respectively.

\section{Real-time PCR}

Animal liver and adipose tissue samples were homogenized and total RNA was extracted using the RNA-STAT 60 protocol (Tel-Test, Austin, TX, USA). RNA purity and quantity were assessed with the Nanodrop (ND-1000, Thermo Scientific, Wilmington, DE, USA) using the ND-1000 V3.8.1 software. cDNA was synthesized from total RNA using the QuantiTect Reverse Transcription Kit (Qiagen, Valencia, CA, USA). Polymerase Chain Reaction (PCR) was performed on the Applied Biosystems StepOnePlus Real-Time PCR Systems using the Taqman Universal PCR Master Mix (Life Technologies, 
Carlsbad, CA, USA). Primer sequences for Taqman Gene Expression Assays (Applied Biosystems, Foster City, CA) were as follows: tumor necrosis factor alpha (TNFa); (Mm00443258-m1), fatty acid synthase (FAS); (Mm00662319m1), peroxisome proliferator-activated receptor alpha (PPARa); (Mm00440939m1), carnitine palmitoyl transferase 1A (CPT1A); (Mm01231183-m1), sterol regulatory element binding protein (SREBP-1C); (Mm00550338-m1), cytochrome P450s [Cyp4a10 (Mm02601690-gH), Cyp2b10 (Mm01972453-s1), Cyp3a11 (Mm007731567-m1), Cyp1a2 (Mm00487224-m1)], CD36 (Mm01135198-m1), phosphoenolpyruvate carboxy kinase (PEPCK-1); (Mm01247058-m1), stearoyl coenzyme A desaturase1 (SCD1); (Mm00772290-m1), interleukin 6 (IL-6); (Mm00446190-m1), monocyte inducible protein 1 (MIP1); (Mm00441258-m1), monocyte chemo attractant protein 2 (MCP2); (Mm01297183-m1), transforming growth factor-beta (TGF $\beta) ;\left(M m 01178820 \_m 1\right)$, collagen 1a1 (Mm00801666_g1), tissue inhibitor of metalloproteinases-1 (TIMP-1); (Mm00441818_m1) and glyceraldehyde-3-phosphate dehydrogenase (GAPDH); (4352932E). The levels of mRNA were normalized relative to the amount of GAPDH mRNA, and expression levels in mice fed control diet and administered vehicle were set at 1 . Gene expression levels were calculated according to the $2^{-\Delta \Delta C t}$ method (92).

\section{Statistical Analysis}

Statistical analyses were performed using GraphPad Prism version 5.01 for Windows (GraphPad Software Inc., La Jolla, CA, USA). Data are expressed as mean \pm SEM. Multiple group data were compared using One Way ANOVA 
followed by Bonferroni's post-hoc test (for parametric data) or Kruskal-Wallis test followed by Dunn's Multiple Comparison Test (for nonparametric data). $P<0.05$ was considered statistically significant. 


\section{RESULTS}

Aroclor 1260 decreased body weight and visceral adiposity in mice fed high fat diet, but had no effect in mice fed control diet

During the 12-week study, body weight gain was measured and percent (\%) increase in bodyweight was calculated (Fig. 2.2 A\&B). All the HFD groups experienced weight gain vs. CD groups. Aroclor 1260 administration did not further increase the body weight of mice consuming HFD. Rather, Aroclor 1260 at $200 \mathrm{mg} / \mathrm{kg}$ decreased the body weight gain in HFD-fed mice vs. HFD+vehicle group $(171.7 \pm 8.9 \%$ vs. $156.9 \pm 11.3 \%, p<0.05)$. Aroclor 1260 at $20 \mathrm{mg} / \mathrm{kg}$ had no effect on body weight in HFD or CD groups. The \% body fat composition was evaluated by DEXA scanning prior to harvesting the animals (Fig. 2.2 C). HFD consumption increased total body fat. Aroclor 1260 at both doses diminished the increase in $\%$ body fat composition in HFD-fed mice $(p<0.05)$ but not in CD-fed mice. In fact, mice co-exposed to HFD and Aroclor $1260(20 \mathrm{mg} / \mathrm{kg})$ appeared to have an increase in lean body mass as compared to HFD-fed mice only (Fig. 2.2 D). There were no differences in \% fat composition in CD-fed mice exposed to Aroclor 1260 vs. CD+vehicle group. The pattern of epididymal fat to body weight ratio among the six groups was similar to the pattern of body fat composition obtained by DEXA scanning, further supporting the observation that Aroclor 1260 exposure attenuated the increase in \% body fat caused by HFD feeding (Fig. 2.2 E).

Mean epididymal adipocyte area $\left(\mu \mathrm{m}^{2}\right)$ was larger in HFD+vehicle $v s$. CD+vehicle $(p<0.05)$. However, Aroclor 1260 had no effect at any of the doses 
administered, either in mice fed CD or HFD (Fig. 2.2 F\&G). Food consumption per mouse per day (g) was calculated over the 12-week period of study. Although all the HFD groups of animals consumed more food (approximately 1.7-fold more calories) as compared to CD groups, Aroclor 1260 did not alter the food consumption rate (Fig. 2.2 H). Thus, Aroclor 1260 exposure at both doses did not increase diet-induced adiposity or adipocyte size. 

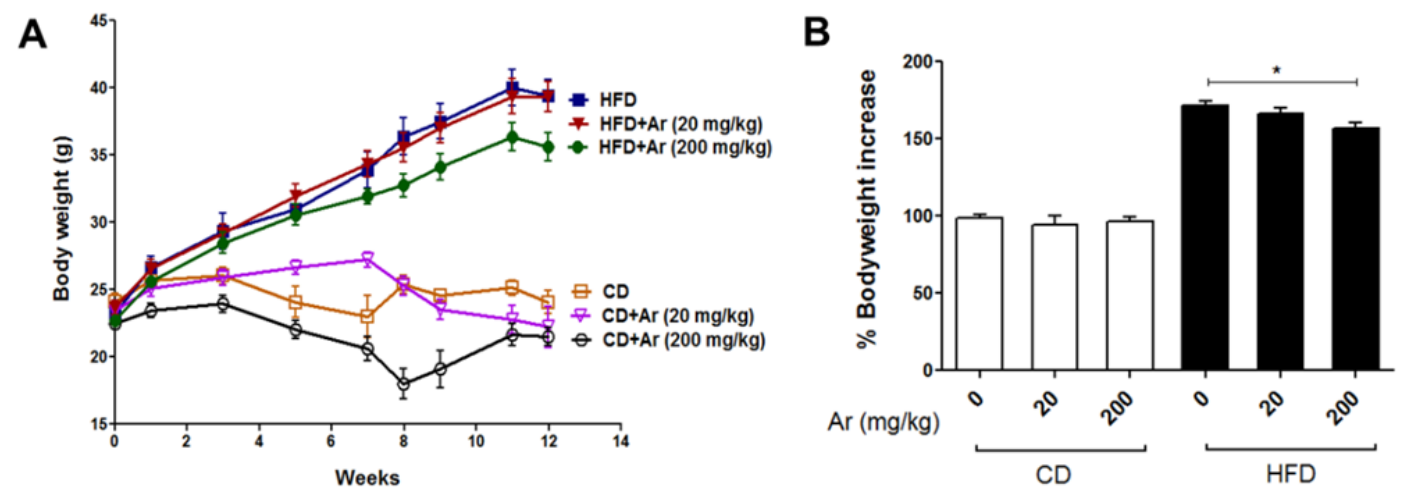

C

D

E
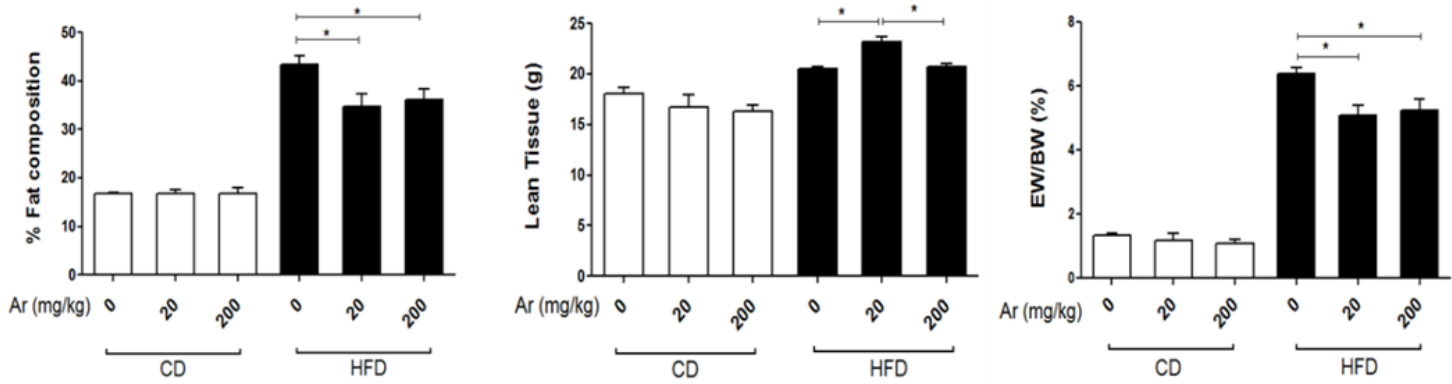

$\mathbf{F}$

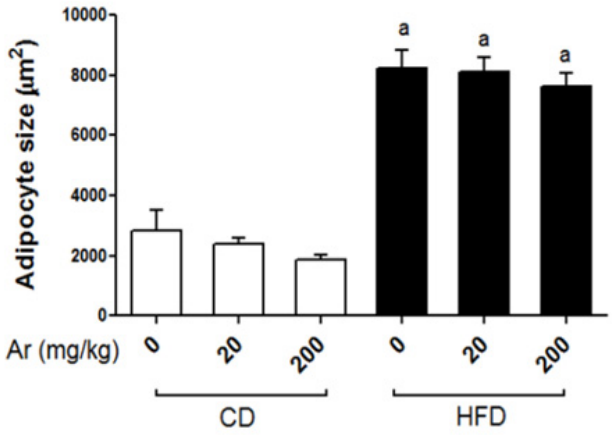

G
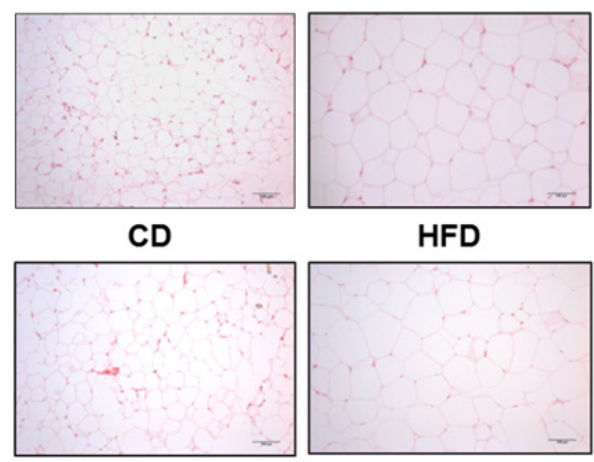

HFD

H

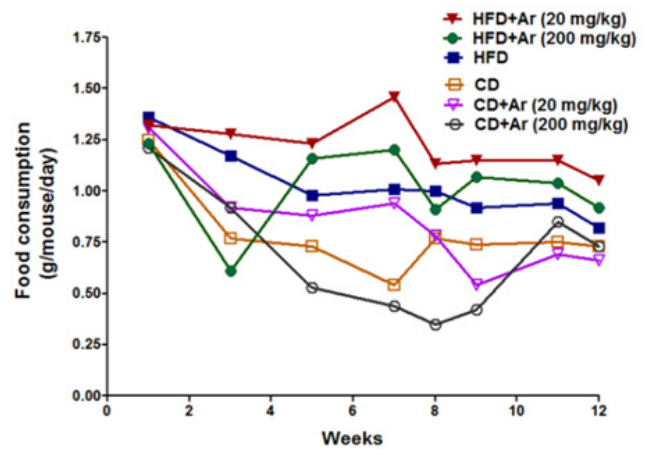

$\mathrm{CD}+\mathrm{Ar}(20 \mathrm{mg} / \mathrm{kg}) \quad \mathrm{HFD}+\operatorname{Ar}(20 \mathrm{mg} / \mathrm{kg})$
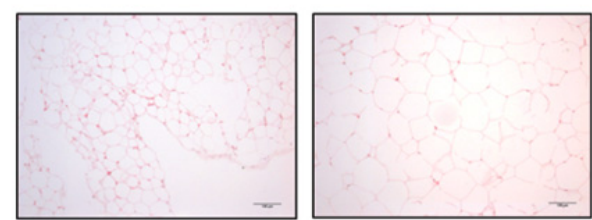

$C D+A r(200 \mathrm{mg} / \mathrm{kg}) \quad H F D+A r(200 \mathrm{mg} / \mathrm{kg})$ 
Figure 2.2. Effects of Aroclor 1260 exposure on body weight and visceral adiposity.

(A) Increase in body weight with time for C57BL/6 mice $(n=10)$ fed with a $42 \%$ milk fat diet (vs. CD). Body weight measurements were taken from Week 1 to Week 12 (12 weeks). (B) The \% increase in body weight gain with time was calculated and the body weight at Week 1 was taken as $100 \%$. (C) The $\%$ fat composition was measured using the Lunar PIXImus densitometer (DEXA scanner). Aroclor 1260 (20 and $200 \mathrm{mg} / \mathrm{kg}$ ) exposure lowered \% fat composition in HFD-fed mice vs. HFD+vehicle group. (D) The lean tissue mass (g) was also measured using the DEXA scanner. (E) Epididymal weight (EW) to body weight (BW) ratio was calculated for all groups of mice. (F \& G) Epididymal adipose tissue was stained with H\&E. and the adipocyte size $\left(\mu \mathrm{m}^{2}\right)$ was measured. The average cell size of $>100$ cells for each group was calculated. $(H)$ Food consumption throughout the 12 -week study was measured. Values are mean \pm SEM, $p<0.05$, a- $\Delta$ due to HFD. CD-control diet, HFD-high fat diet, Ar-Aroclor 1260. Figure adapted from Wahlang et al., Toxicol Appl Pharmacol, 279 (3), 2014. 


\section{Aroclor 1260 exposure caused increased liver injury in HFD-fed mice}

CD-fed mice with or without Aroclor 1260 exposure did not develop steatosis or steatohepatitis as seen with H\&E staining of the liver sections (Fig. 2.3 A). Mice fed with HFD developed steatosis in both the unexposed and Aroclor 1260-exposed groups. The most striking difference between the HFD and HFD+Aroclor 1260 groups was transformation from steatosis to steatohepatitis. HFD+Aroclor 1260 co-exposures resulted in scattered foci of mononuclear cells and neutrophils (confirmed with CAE stained slides) and hepatocyte necrosis which appeared greater in the high dose group (Fig. 2.3 A\&B). Aroclor 1260 exposure was associated with centrilobular enlargement of hepatocytes and karyomegaly irrespective of diet type, possibly reflecting hepatic enzyme induction in these mice. Liver to body weight ratio was calculated and there were no differences among the groups (Fig. 2.3 C). No inflammation was observed in Aroclor 1260-exposed mice fed CD.

Liver injury, as determined by elevated serum ALT levels, was most noticeable in the HFD+Aroclor $1260(20 \mathrm{mg} / \mathrm{kg})$ group $(p<0.05)$ while serum AST was unaffected (Fig. 2.3 D\&E). Although the higher exposure group $(200 \mathrm{mg} / \mathrm{kg})$ fed HFD tended to have more inflammatory foci based on histological assessment, serum ALT levels were not significantly elevated in this group. In the CD-fed mice, Aroclor 1260 exposure did not alter the serum ALT and AST levels at either dose. Hepatic expression of fibrotic markers including transforming growth factor-beta (TGF $\beta$ ), collagen $1 \alpha 1$ and tissue inhibitor of 
metalloproteinases-1 (TIMP-1) were measured (Fig. 2.3 F,G\&H) and there was no increase in mRNA levels among the HFD groups.

The HFD+vehicle group showed a significant increase in hepatic levels of cholesterol and triglycerides vs. any of the CD groups (Fig. 2.4 A\&B). However, hepatic cholesterol and triglycerides levels were not significantly increased in the HFD+Aroclor 1260 at 20 or $200 \mathrm{mg} / \mathrm{kg}$ vs. any of the CD groups. Likewise, hepatic cholesterol and triglycerides levels were not significantly increased in the HFD+Aroclor 1260 at 20 or $200 \mathrm{mg} / \mathrm{kg}$ vs. the HFD group alone. Rather, there was a trend towards decreased hepatic cholesterol and triglycerides with Aroclor 1260 exposure in HFD-fed mice but this was not significant. Taken together, these results suggest that Aroclor 1260 exposure did not worsen steatosis caused by HFD but augmented inflammation and induced steatohepatitis instead. 


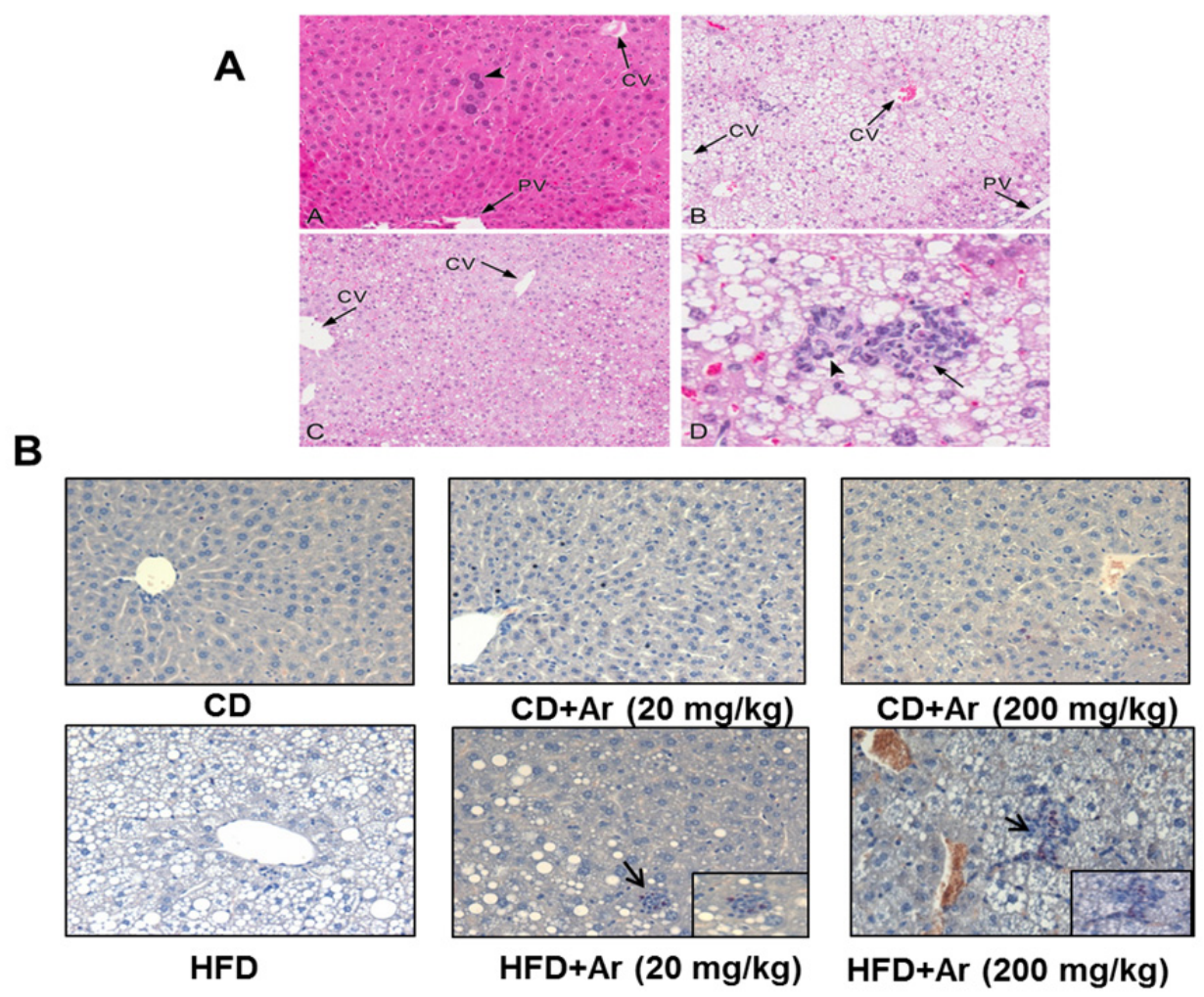

C

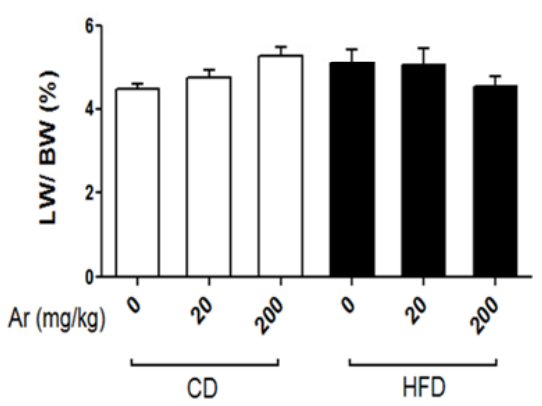

E

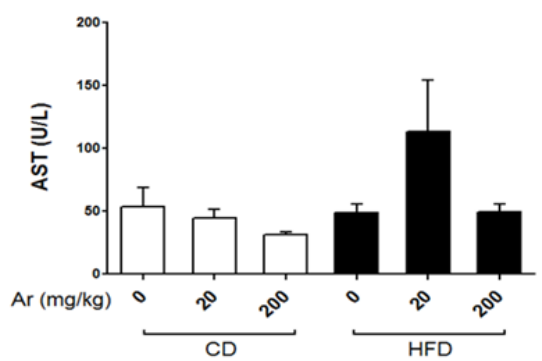

G

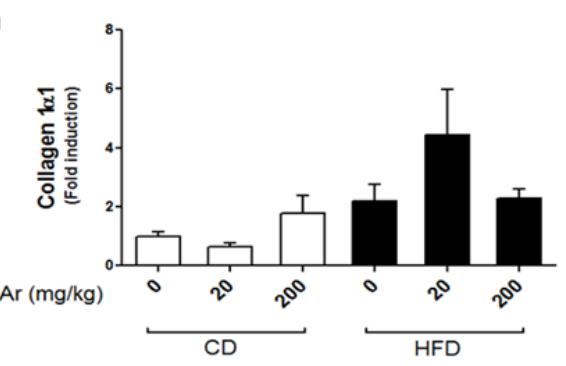

D

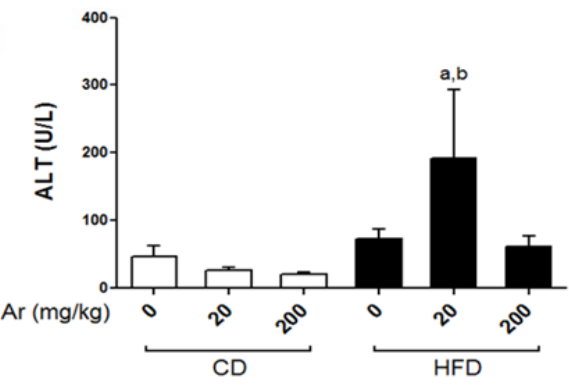

F

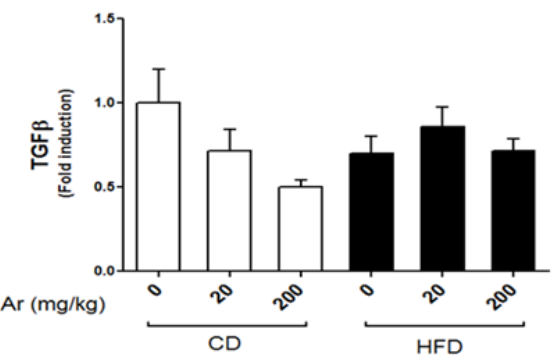

H

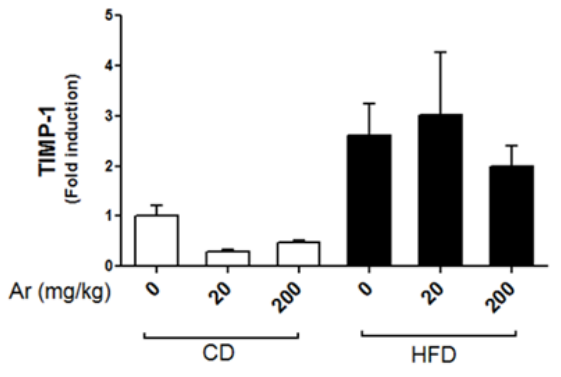


Figure 2.3. Aroclor 1260 exposure caused steatohepatitis in HFD-fed mice but had no effect on fibrotic markers.

(A) H\&E staining of hepatic sections established the occurrence of centrilobular hepatocellular hypertrophy, karyomegaly, and multinucleate (arrow head) hepatocytes in the CD+Aroclor $1260(200 \mathrm{mg} / \mathrm{kg})$ group [A]. HFD consumption resulted in variable, centrilobular, microvesicular lipidosis [B-D], while centrilobular hepatocellular hypertrophy was observed in HFD+Aroclor $1260(20 \mathrm{mg} / \mathrm{kg})$ mice [C]. HFD-fed mice exposed to Aroclor $1260(200 \mathrm{mg} / \mathrm{kg})$ exhibited occasional, small areas of necrosis and inflammation (steatohepatitis) [D], characterized by neutrophils (arrow head) and pyknotic debris (arrow). (B) CAE staining demonstrated neutrophil infiltration in the HFD+Aroclor 1260 (20 and $200 \mathrm{mg} / \mathrm{kg}$ ) groups. (C) The liver weight (LW) to body weight (BW) ratio was calculated. (D) Serum ALT and (E) AST levels (U/L) were measured $(n=10)$ using the Piccolo Xpress chemical analyzer. Real-time PCR experiments were performed to measure the hepatic expression of $(F) T G F \beta,(G)$ Collagen1a1 and (H) TIMP-1, $n=10$. Values are mean \pm SEM, $p<0.05$, a- $\Delta$ due to HFD, b- $\Delta$ due to Aroclor 1260 exposure at $20 \mathrm{mg} / \mathrm{kg}$. CD-control diet, HFD-high fat diet, ArAroclor 1260, PV-portal vein, CV-central vein. Figure adapted from Wahlang et al., Toxicol Appl Pharmacol, 279 (3), 2014. 
A

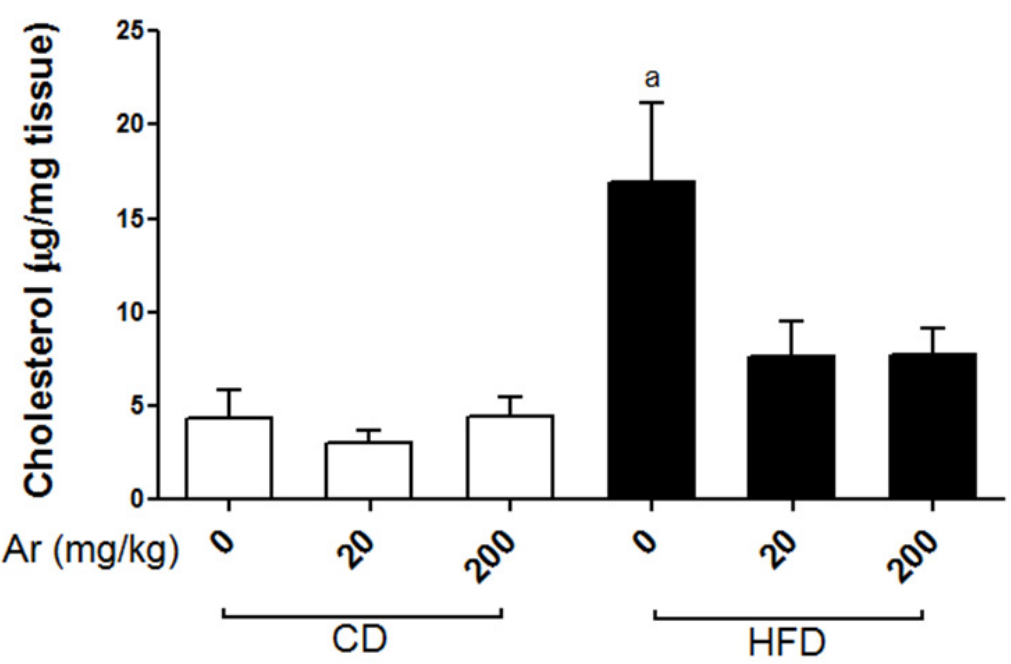

B

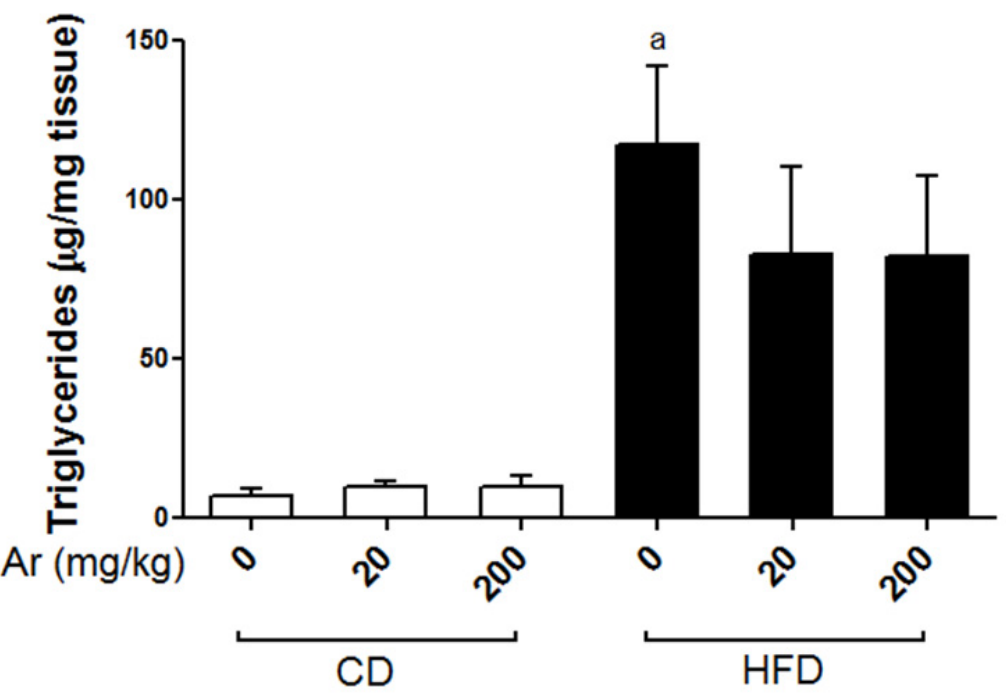


Figure 2.4. Effects of Aroclor 1260 exposure on hepatic cholesterol and triglycerides.

Hepatic levels of $(A)$ cholesterol and $(B)$ triglycerides were quantified ( $\mu \mathrm{g} / \mathrm{mg}$ tissue) in mice $(\mathrm{n}=5)$ fed with CD or HFD with or without Aroclor 1260 coexposure. Values are mean \pm SEM, $p<0.05$, a- $\Delta$ due to HFD. CD-control diet, HFD-high fat diet, Ar-Aroclor 1260. Figure adapted from Wahlang et al., Toxicol Appl Pharmacol, 279 (3), 2014. 


\section{Effects of Aroclor 1260 exposure on serum adipo-cytokines}

HFD consumption alone did not increase serum interleukin 6 (IL-6) or tissue plasminogen activator inhibitor (tPAl-1) levels vs. CD (Fig. 2.5 A\&B). However, HFD-fed mice exposed to Aroclor 1260 at $20 \mathrm{mg} / \mathrm{kg}$ displayed significantly elevated serum IL-6 and tPAI-1 levels $(p<0.05)$. This exposurerelated elevation in pro-inflammatory cytokines was absent in CD-fed mice. In contrast to the lower exposure, Aroclor 1260 at $200 \mathrm{mg} / \mathrm{kg}$ did not increase serum IL-6 or tPAI-1 levels in either the HFD or CD groups even though histological examinations indicated higher levels of hepatic macrophage infiltration in HFD fed animals. Serum tumor necrosis factor alpha (TNFa) and monocyte chemo attractant protein-1 (MCP1) levels were not affected by either diet or Aroclor 1260 exposure (Fig. 2.5 C\&D).

All the HFD groups displayed elevated serum leptin levels as compared to CD groups (Fig. $2.5 \mathrm{E}, p<0.05$ ) consistent with the increased adiposity observed in these groups. Exposure to Aroclor 1260 did not affect leptin levels. Interestingly, serum adiponectin levels did not differ between any of the groups, irrespective of the diet type consumed (Fig. $2.5 \mathrm{~F}$ ), leading to an increased leptin/adiponectin ratio in HFD-fed animals. Similar to leptin, serum resistin levels were increased in mice fed HFD (Fig. $2.5 \mathrm{G}, p$ <0.05). Furthermore, Aroclor 1260 at $200 \mathrm{mg} / \mathrm{kg}$ decreased serum resistin levels in HFD-fed mice vs. HFD+vehicle and HFD+Aroclor $1260(20 \mathrm{mg} / \mathrm{kg})$ groups $(p<0.05)$, indicating a dosedependent interaction between Aroclor 1260 and HFD. In summary, Aroclor 1260 
at the lower dose increased serum pro-inflammatory cytokines in HFD-fed mice, while the higher dose caused a decrease in serum resistin levels. 
A

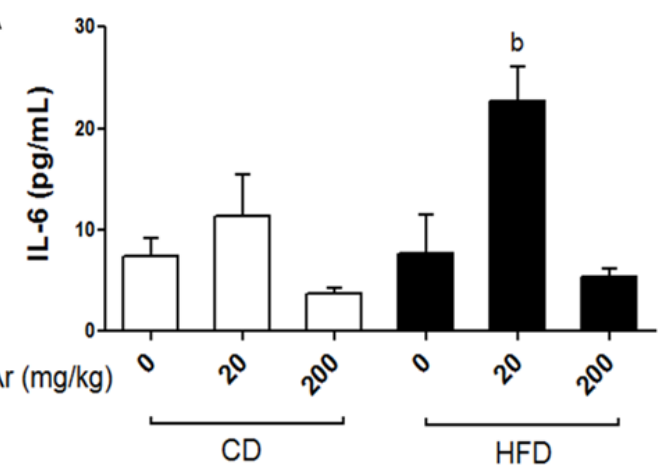

C

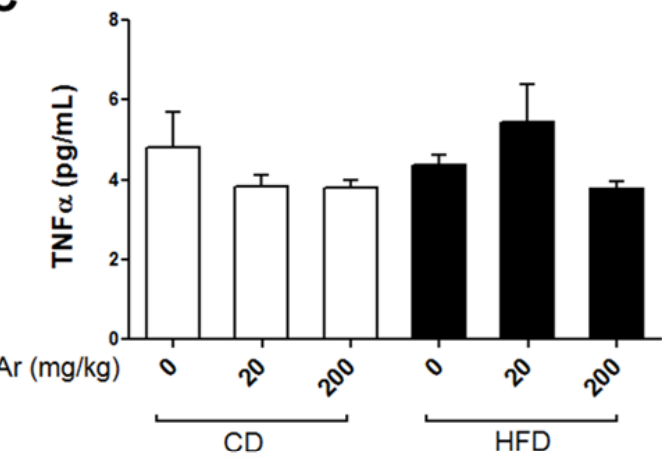

E

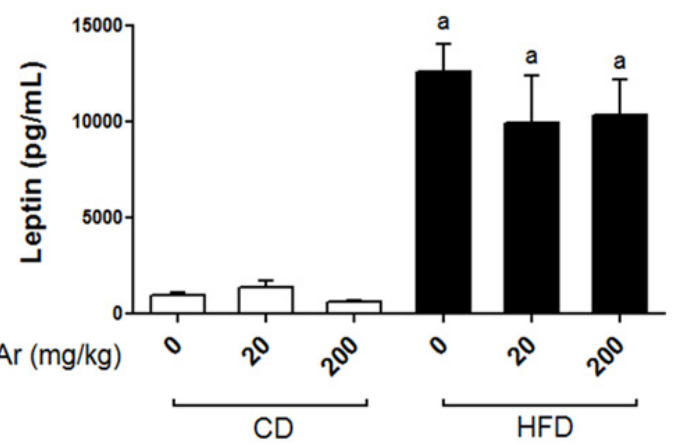

B

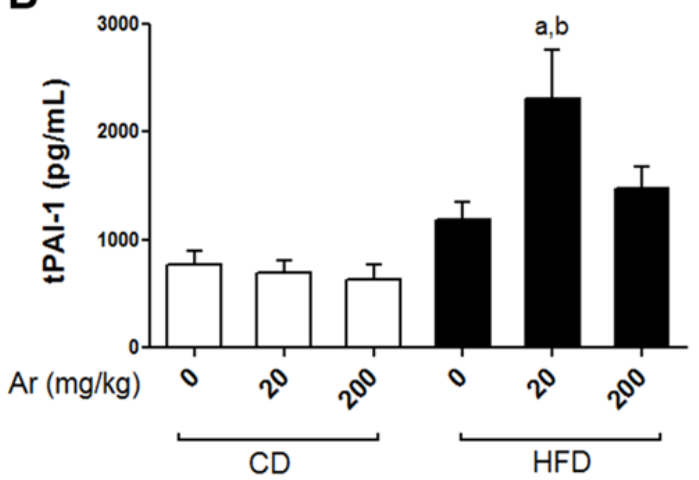

D

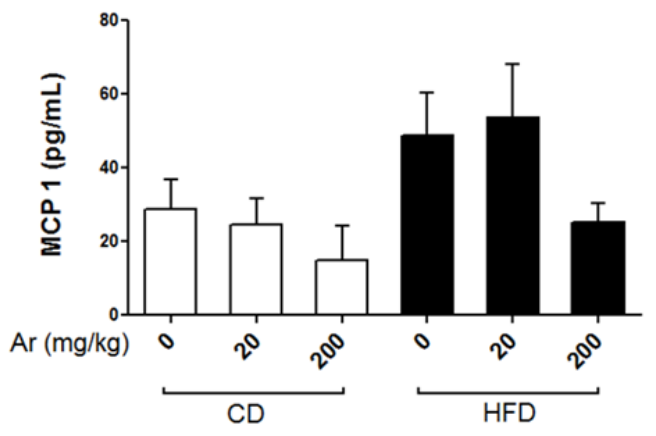

F

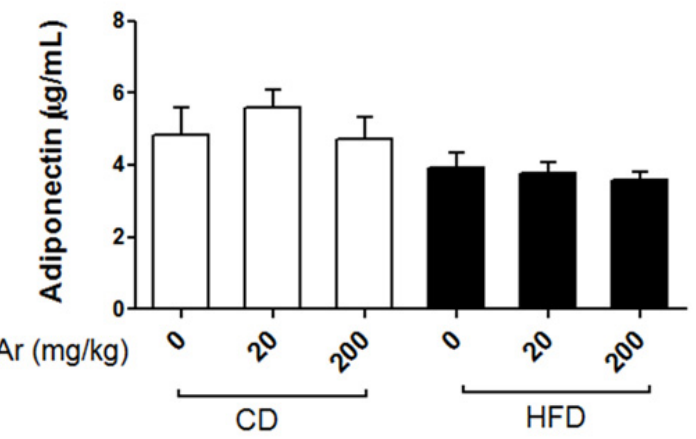

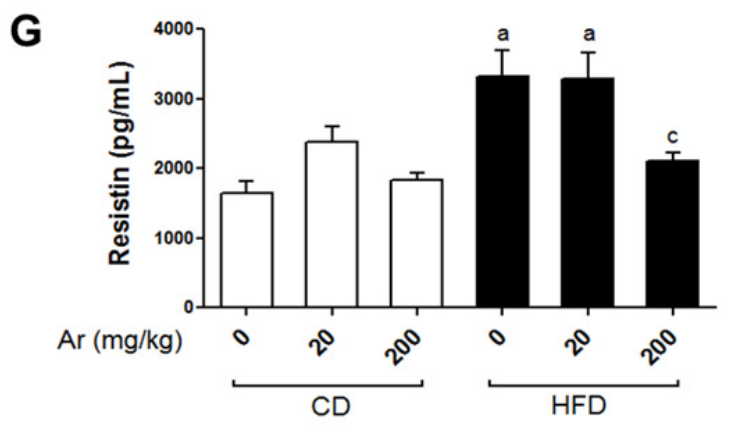


Figure 2. 5. Effects of Aroclor 1260 on serum adipo-cytokines.

Serum (A) IL-6 (pg/mL), (B) tPAl-1 (pg/mL), (C) TNFa (pg/mL), (D) MCP1 $(\mathrm{pg} / \mathrm{mL}),(E)$ leptin $(\mathrm{pg} / \mathrm{mL}),(\mathrm{F})$ adiponectin $(\mu \mathrm{g} / \mathrm{mL})$ and $(\mathrm{G})$ resistin $(\mathrm{pg} / \mathrm{mL})$ levels were measured using the Luminex IS 100 system $(n=10)$. Values are mean \pm SEM, $p<0.05$, a- $\Delta$ due to HFD, b- $\Delta$ due to Aroclor 1260 exposure at 20 $\mathrm{mg} / \mathrm{kg}, \mathrm{c}-\Delta$ due to Aroclor 1260 exposure at $200 \mathrm{mg} / \mathrm{kg}$. CD-control diet, HFDhigh fat diet, Ar-Aroclor 1260. Figure adapted from Wahlang et al., Toxicol Appl Pharmacol, 279 (3), 2014. 
Effects of Aroclor 1260 on insulin resistance, glucose regulation, and serum lipids

A glucose tolerance test was performed and HOMA-IR was calculated to determine if HFD and Aroclor 1260 co-exposure exacerbate HFD-induced insulin resistance, a common hallmark of NAFLD. HFD feeding increased HOMA-IR and Aroclor 1260 exposure (200 mg/kg) decreased HOMA-IR in HFD-fed mice (Fig. 2.6 A). The HFD group showed greater area under the curve (AUC) in the glucose tolerance test than any of the CD groups (Fig. 2.6 B\&C). Aroclor 1260 though had no additive effect to dietary manipulation alone. Therefore, while Aroclor 1260 exposure $(200 \mathrm{mg} / \mathrm{kg})$ decreased HOMA-IR, there was no difference in the AUC. The decrease in HOMA-IR could indicate impaired glucose-stimulated insulin production with Aroclor 1260 exposure. Fasting blood glucose levels showed that Aroclor 1260 exposure lowered blood glucose levels in the CD-fed mice at both the 20 and $200 \mathrm{mg} / \mathrm{kg}$ doses but not in HFD-fed mice (Fig. 2.6 D). This observation is consistent with our earlier study where i.p. injection of PCB 153 resulted in lowered fasting blood glucose levels in mice fed $\mathrm{CD}(48)$.

HFD feeding resulted in significantly higher mean serum cholesterol and HDL levels vs. CD feeding $(p<0.05)$. Aroclor 1260 exposure at 20 or $200 \mathrm{mg} / \mathrm{kg}$ did not affect mean cholesterol and HDL levels either in the HFD or CD groups (Table 3). In contrast, HFD feeding did not increase mean serum LDL levels vs. CD feeding. However, the HFD+Aroclor $1260(200 \mathrm{mg} / \mathrm{kg})$ group exhibited significantly higher mean LDL levels vs. CD+vehicle or CD+Aroclor 1260 (200 
$\mathrm{mg} / \mathrm{kg}$ ). Mean serum triglyceride levels were unchanged irrespective of diet consumed or level of Aroclor 1260 exposure. In summary, HFD was associated with insulin resistance and serum hypercholesterolemia, but Aroclor 1260 had no observable effect on these parameters. 

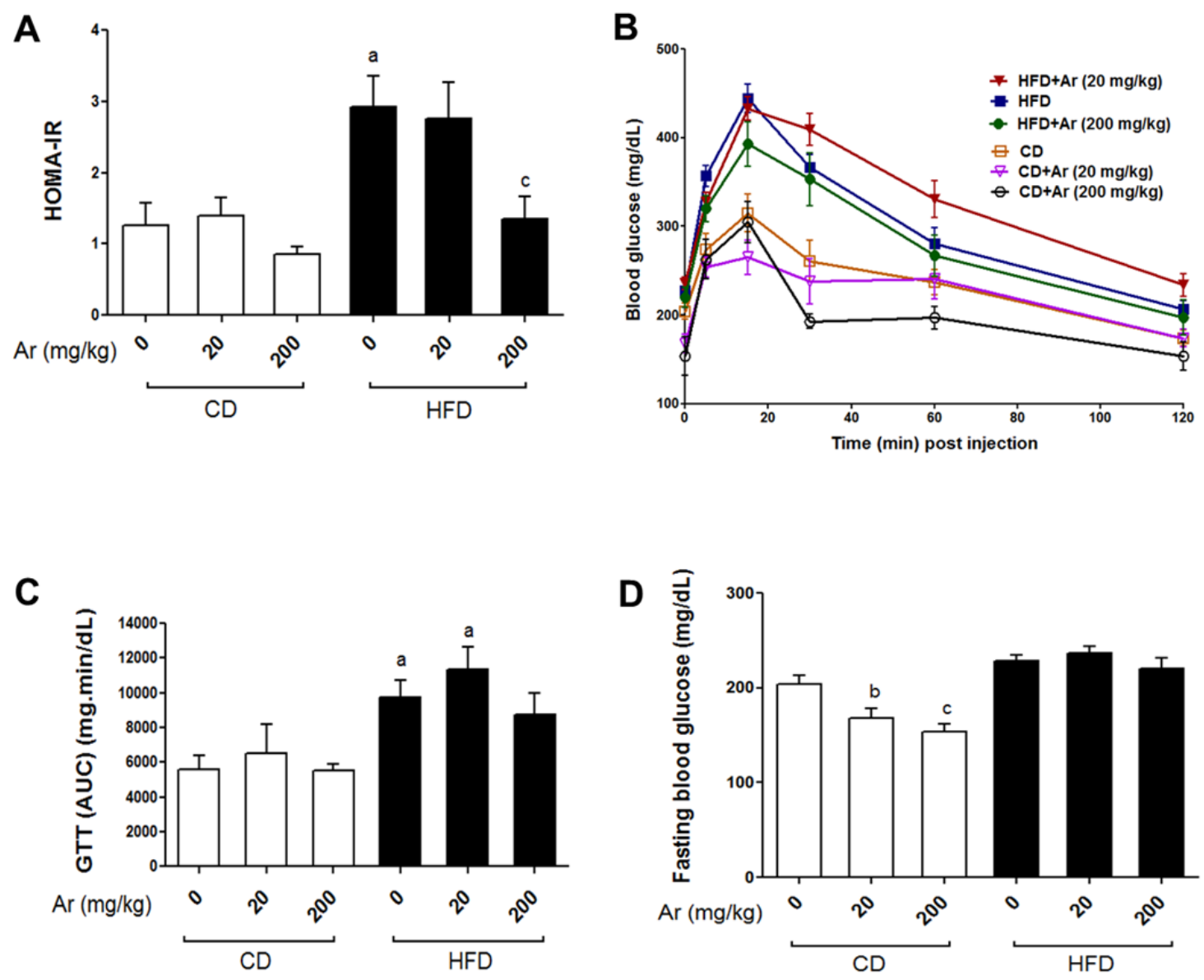
Figure 2.6.HFD increased insulin resistance, and this was unaffected by Aroclor 1260 co-exposure.

(A) HOMA-IR was calculated from fasting blood glucose and insulin levels for all six groups of animals ( $n=10)$. (B) Glucose tolerance test was performed, and blood glucose levels were measured for mice $(n=10)$ fed with CD or HFD, with or without Aroclor 1260 co-exposure. AUC was calculated (C), and the HFD groups showed higher AUC levels than the CD groups. (D) Fasting blood glucose levels (mg/dL) were measured, and the CD-fed mice co-exposed to Aroclor 1260 showed lower levels vs. CD+vehicle.Values are mean \pm SEM, $p<0.05$, a- $\Delta$ due to HFD, b- $\Delta$ due to Aroclor 1260 exposure at $20 \mathrm{mg} / \mathrm{kg}$, c- $\Delta$ due to Aroclor 1260 exposure at $200 \mathrm{mg} / \mathrm{kg}$. CD-control diet, HFD-high fat diet, Ar-Aroclor 1260.

Figure adapted from Wahlang et al., Toxicol Appl Pharmacol, 279 (3), 2014. 
CD

$\mathrm{CD}+\mathrm{Ar}$

(20 mg/kg)

$\mathrm{CD}+\mathrm{Ar}$

(200 mg/kg)

HFD

HFD+Ar

(20 $\mathrm{mg} / \mathrm{kg}$ )

HFD+Ar (200

$\mathrm{mg} / \mathrm{kg}$ )
$70.1 \pm 7.7$

$49.1 \pm 6.1$

$12.3 \pm 1.7$

$44.1 \pm 6.1$

$$
76.0 \pm 3.9
$$

$52.2 \pm 3.0$

$16.1 \pm 1.3$

$40.2 \pm 3.6$

$76.8 \pm 3.8$

$56.0 \pm 3.0$

$12.0 \pm 1.6$

$43.8 \pm 6.3$

$122.1 \pm 11.4^{a}$

$96.8 \pm 9.8^{a}$

$17.3 \pm 1.8$

$38.6 \pm 3.1$

$$
130.4 \pm 8.2^{a}
$$

$92.2 \pm 4.4$

$15.5 \pm 2.3$

$53.0 \pm 3.3$

$130.0 \pm 5.3^{a}$

$93.1 \pm 2.7^{a}$

$50.0 \pm 3.1$

Table 3. Serum levels of cholesterol, high density lipoproteins, low density lipoproteins and triglycerides.

Values are mean \pm SEM $(\mathrm{mg} / \mathrm{dL}), p<0.05$, a- $\Delta$ due to HFD, b- $\Delta$ due to Aroclor 1260 exposure at $20 \mathrm{mg} / \mathrm{kg}$, c- $\Delta$ due to Aroclor 1260 exposure at 200 mg/kg. CD-control diet, HFD-high fat diet, Ar-Aroclor 1260, HDL-high density lipoproteins, LDL-low density lipoproteins. Table adapted from Wahlang et al., Toxicol Appl Pharmacol, 279 (3), 2014. 


\section{Aroclor 1260 exposure modulated hepatic fat metabolism}

Liver-X-receptor (LXR) is the key transcription factor that drives lipid synthesis and cholesterol metabolism and its over-activation can promote or worsen steatosis (93). We were interested in evaluating if Aroclor 1260 exposure would increase LXR target gene expression, which in turn, worsens steatosis caused by HFD. There are similarities in the response elements, to which LXR and the receptors that PCBs activate (CAR and PXR), bind to. All three receptors have the capacity to bind direct repeats (DR-4) response elements suggesting that some degree of crosstalk may be possible. HFD feeding did not alter FAS hepatic expression vs. CD feeding (Fig. 2.7 A). However, Aroclor 1260 exposure at 20 and $200 \mathrm{mg} / \mathrm{kg}$ decreased FAS hepatic expression in HFD-fed mice ( $p$ <0.05). HFD feeding increased SCD1 hepatic expression (Fig. $2.7 \mathrm{~B}, p<0.05$ ) and this result was not affected by Aroclor 1260 exposure. Interestingly, HFD feeding alone decreased SREBP-1c mRNA levels but co-exposure to HFD and Aroclor 1260 at both doses resulted in increased SREBP1-c mRNA levels (Fig. 2.7 C, $p<0.05$ ), suggesting an interaction between HFD and Aroclor 1260 exposure. Aroclor 1260 exposure at both doses had no effect on SREBP-1c hepatic expression in CD-fed mice. In contrast, Aroclor 1260 exposure at 20 $\mathrm{mg} / \mathrm{kg}$ resulted in hepatic upregulation of CD36 in CD-fed mice (Fig. 2.7 D, $p$ <0.05). Notably, CD36 is an LXR target gene shared by PXR as well (94). In summary, Aroclor 1260 exposure did not increase FAS or SCD1 hepatic expression, suggesting that LXR was not activated by the exposure but that 
these genes' expression may have been modulated by CAR or PXR directly or indirectly.

PPARa is a transcription factor for lipid-catabolizing genes including CPT1A, CPT2 and Cyp4b10. PPARa activation results in hepatic lipid oxidation and may be protective against steatosis. We measured the hepatic expression of PPAR $\alpha$ as well as its target genes, Cyp4b10 and CPT1A (Fig. 2.8 A, B\&C). The mRNA levels of PPAR a and Cyp4b10 did not differ between any of the groups examined. However, CPT1A expression was significantly induced with low dose Aroclor 1260 exposure in the HFD group vs. HFD alone. Therefore, Aroclor 1260 exposure at $20 \mathrm{mg} / \mathrm{kg}$ appeared to induce CPT1A in a HFD-setting by mechanisms independent of direct PPARa interaction. 
A B
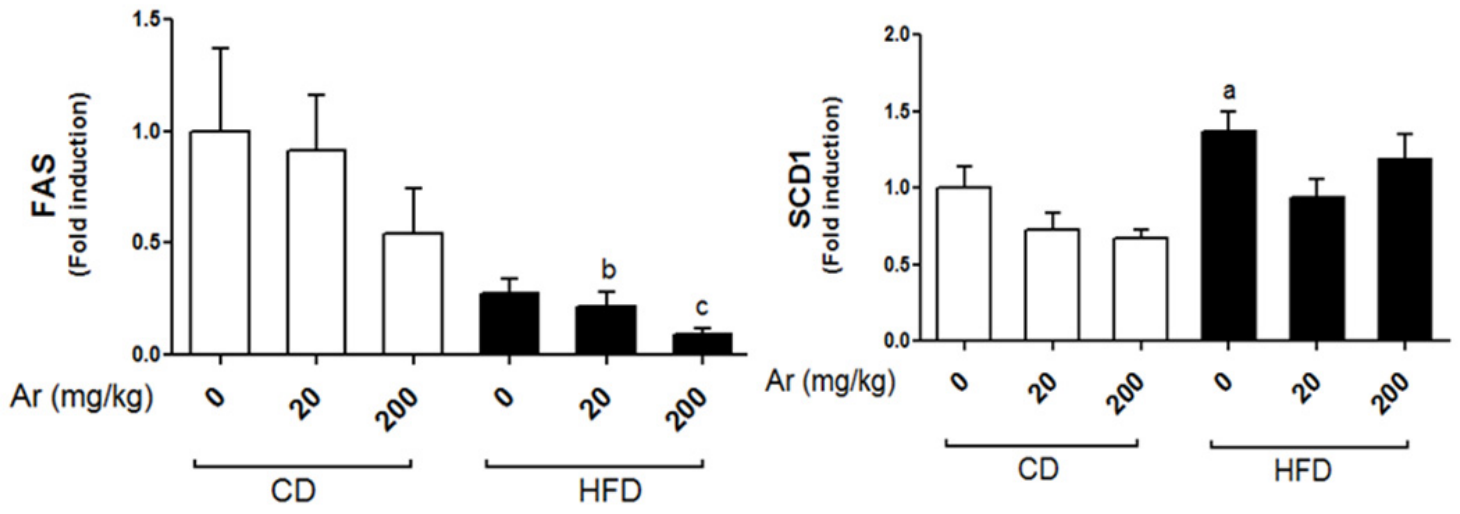

C

D
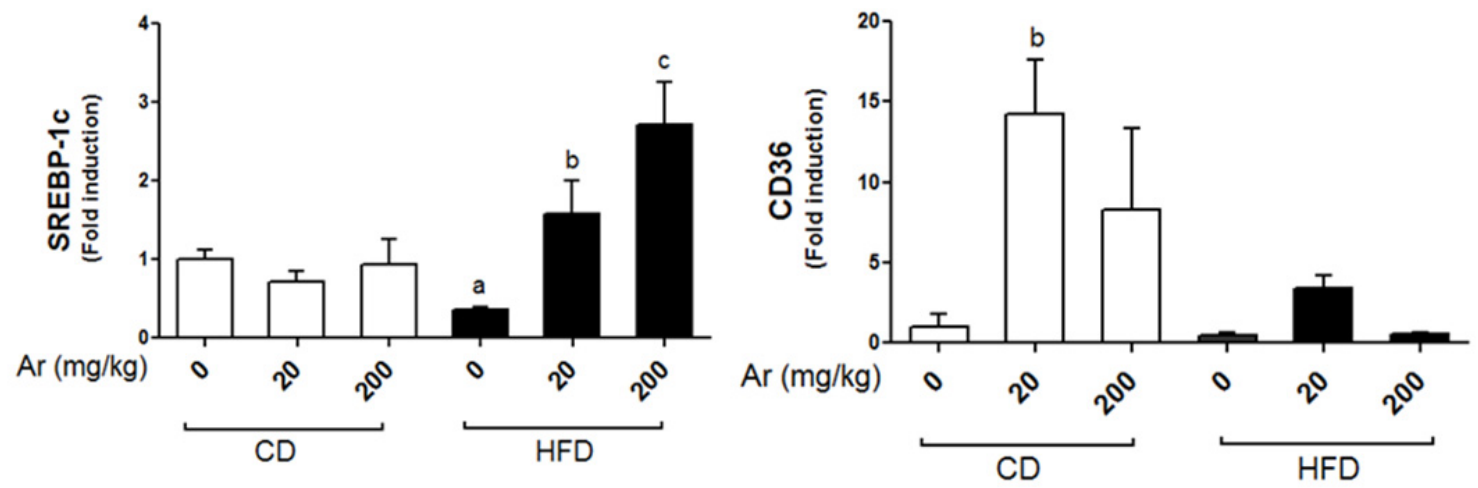
Figure 2.7. Effects of Aroclor 1260 exposure on genes involved in lipid metabolism.

Real-time PCR experiments showed the changes in hepatic mRNA expressions caused by Aroclor 1260 exposure for (A) FAS, (B) SCD1, (C) SREBP-1C and (D) CD36. Values are mean \pm SEM, $p<0.05$, a- $\Delta$ due to HFD, b$\Delta$ due to Aroclor 1260 exposure at $20 \mathrm{mg} / \mathrm{kg}, \mathrm{c}-\Delta$ due to Aroclor 1260 exposure at $200 \mathrm{mg} / \mathrm{kg}$. CD-control diet, HFD-high fat diet, Ar-Aroclor 1260. Figure adapted from Wahlang et al., Toxicol Appl Pharmacol, 279 (3), 2014. 
A

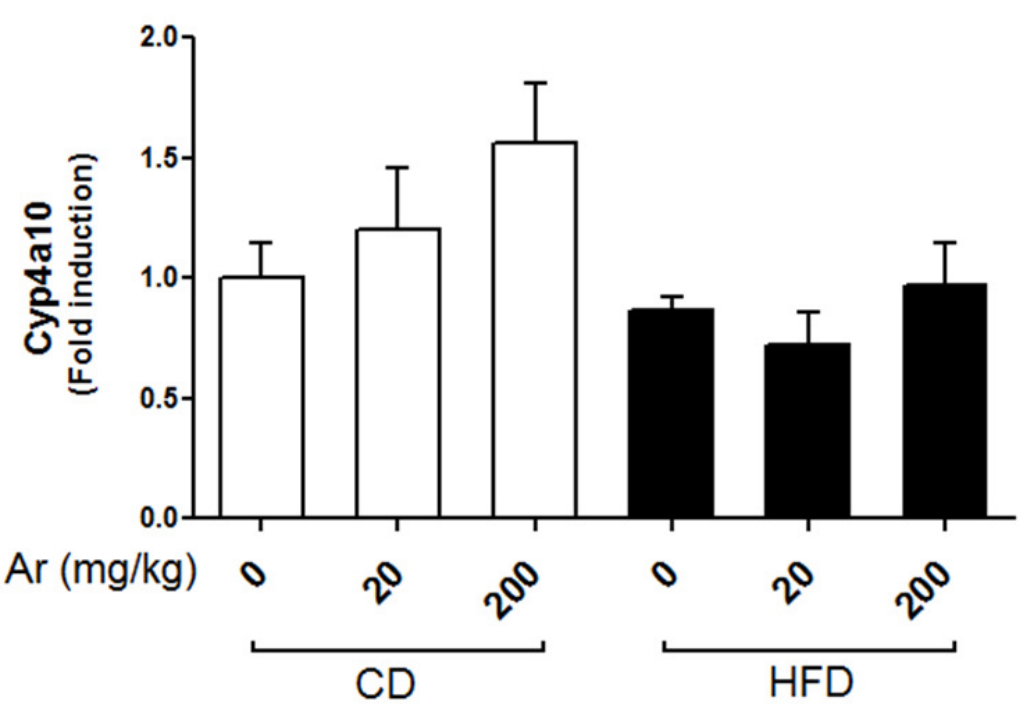

B

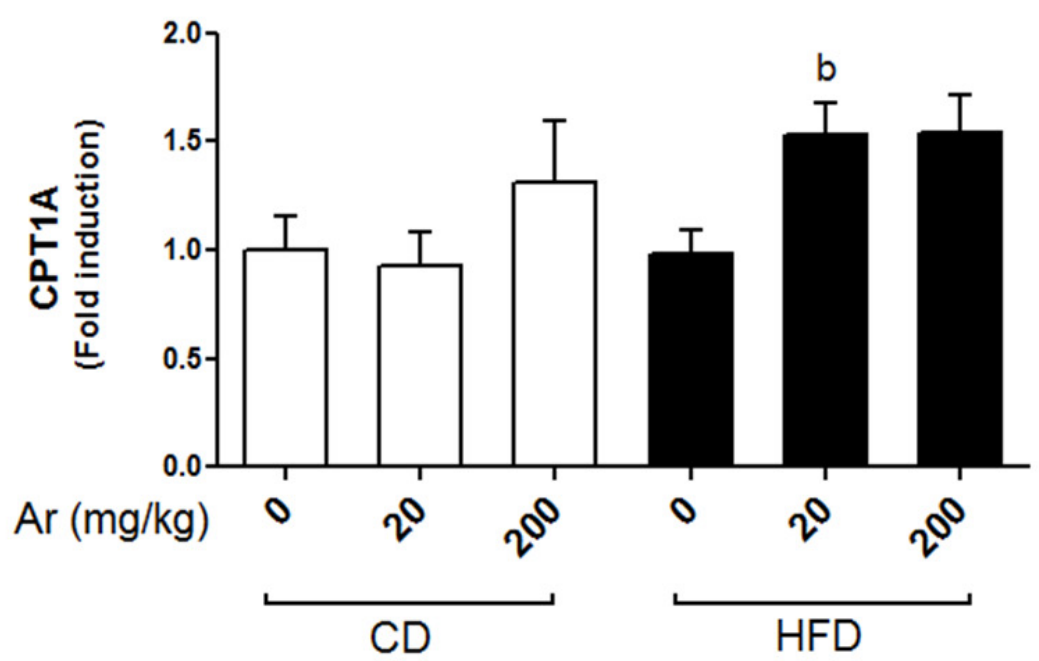

C

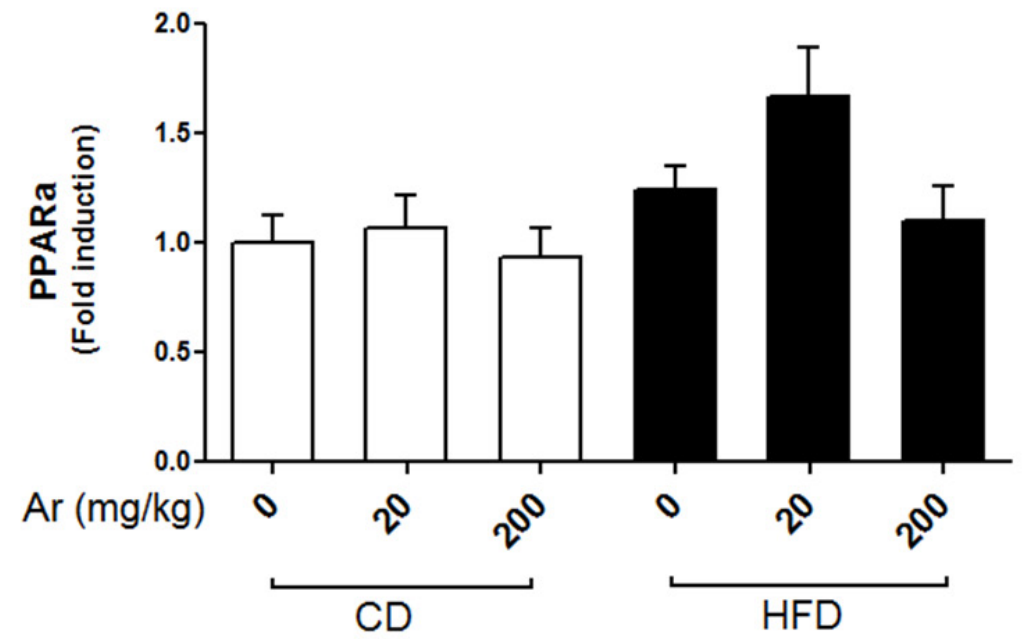


Figure 2.8. Effects of Aroclor 1260 exposure on genes involved in lipid oxidation.

Real-time PCR experiments showed the changes in hepatic mRNA expressions caused by Aroclor 1260 exposure for of (A) Cyp4a10 (B) CPT1A and (C) PPARa. Values are mean \pm SEM, $p<0.05$, b- $\Delta$ due to Aroclor 1260 exposure at $20 \mathrm{mg} / \mathrm{kg}$. CD-control diet, HFD-high fat diet, Ar-Aroclor 1260. Figure adapted from Wahlang et al., Toxicol Appl Pharmacol, 279 (3), 2014. 


\section{Hepatic expression of TLR-4 target genes}

Toll like receptor 4 (TLR-4) activation results in nuclear factor kappa-B (NF-KB) activation which in turn causes upregulation of pro-inflammatory cytokines (95). We hypothesized that Aroclor 1260 may interact directly or indirectly with TLR-4, resulting in monocytic infiltration as observed in liver histology and increased serum cytokine levels. We therefore measured the hepatic expression of TLR-4 target genes namely TNFa and IL-6. HFD alone did not increase TNFa mRNA levels (Fig. 2.9 A). However, exposure to Aroclor 1260 at $20 \mathrm{mg} / \mathrm{kg}$ increased TNFa mRNA levels in HFD-fed mice $(p<0.05)$. Aroclor 1260 exposure at $200 \mathrm{mg} / \mathrm{kg}$ did not increase TNFa hepatic expression in either the CD- or HFD-fed mice. Aroclor 1260 exposure at $20 \mathrm{mg} / \mathrm{kg}$ in CD fed mice resulted in increased IL-6 mRNA levels vs. CD alone (Fig. 2.9 B). Aroclor 1260 exposure at $200 \mathrm{mg} / \mathrm{kg}$ did not increase IL-6 mRNA levels in either the CD- or HFD-fed mice. Clearly, Aroclor 1260 exposure at $20 \mathrm{mg} / \mathrm{kg}$ led to increased TNF $\alpha$ and IL-6 hepatic expressions with either HFD or CD respectively. Neither HFD feeding nor Aroclor 1260 exposure had any effect on MCP2 mRNA levels whereas MIP1 $\alpha$ mRNA levels was increased only with HFD feeding (Fig. 2.9 C\&D). These results appear broadly consistent with the serum cytokine levels data, suggesting increased inflammation and possibly sensitization to TNFadependent cell death only at the lower dose of Aroclor $1260(20 \mathrm{mg} / \mathrm{kg})$.

Furthermore, the results also suggest that cytokine production may be inhibited at higher concentrations of Aroclor $1260(200 \mathrm{mg} / \mathrm{kg})$ by other mechanisms. 
A B
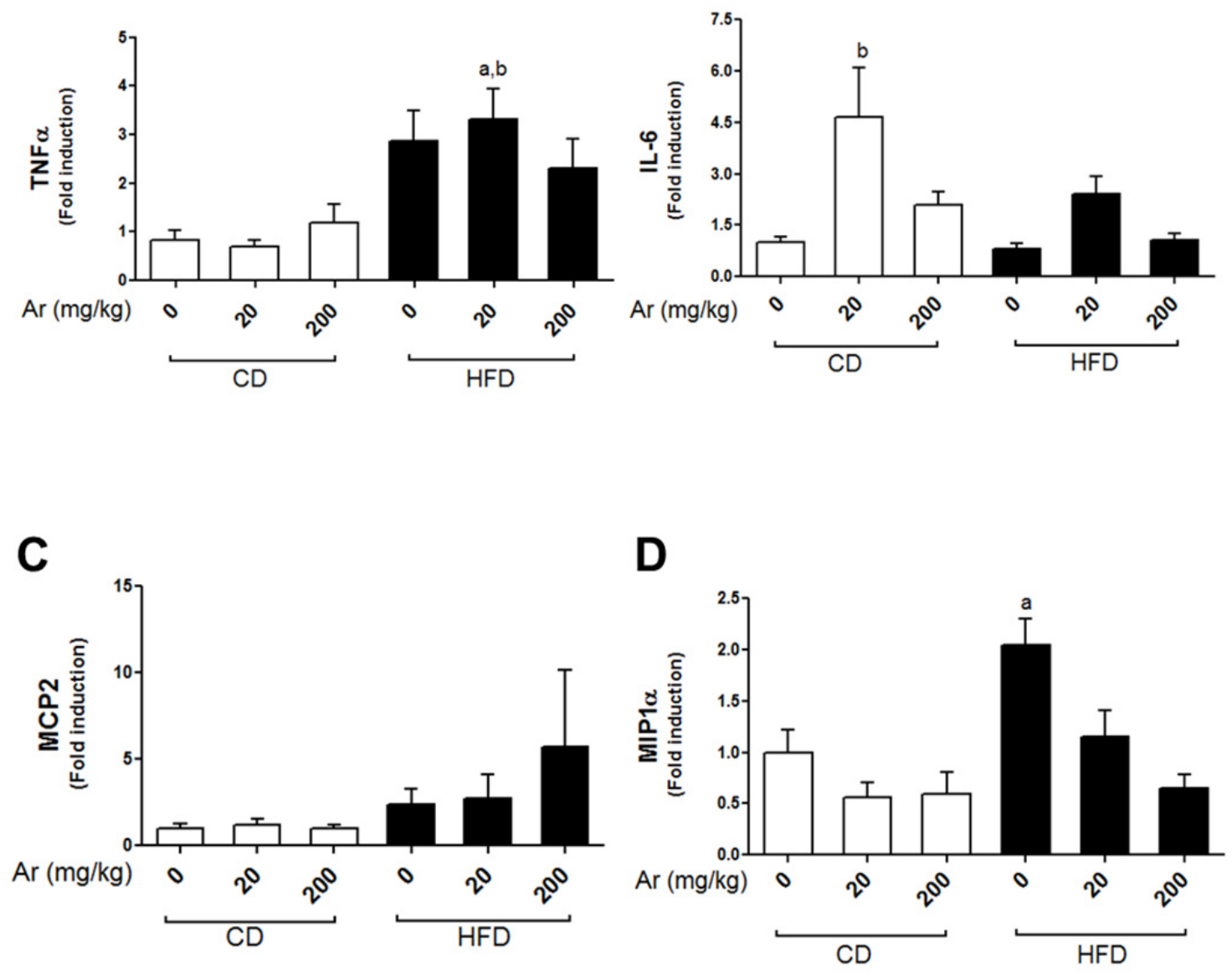
Figure 2.9. Effects of Aroclor 1260 exposure on TLR-4 target genes.

Real-time PCR experiments showed the changes in hepatic mRNA expressions caused by Aroclor 1260 exposure for (A) TNFa, (B) IL-6, (C) MCP2 and (D) MIP1 $\alpha$. Values are mean \pm SEM, $p<0.05$, a- $\Delta$ due to HFD, b- $\Delta$ due to Aroclor 1260 exposure at $20 \mathrm{mg} / \mathrm{kg}$. CD-control diet, HFD-high fat diet, Ar-Aroclor 1260. Figure adapted from Wahlang et al., Toxicol Appl Pharmacol, 279 (3), 2014. 


\section{Aroclor 1260 induced hepatic CAR, PXR and AhR target genes}

PCBs are known activators of AhR and CAR, which are involved in xenobiotic detoxification $(24,96)$. Recent studies have demonstrated PCBs' interaction with other nuclear receptors including human PXR and rodent peroxisome-proliferator activated receptor alpha (PPARa) (97-99). We hypothesized that Aroclor 1260 may interact with these receptors in our animal model. We therefore looked at the hepatic expression levels of these receptors' target genes in all animal groups.

The mRNA levels of Cyp2b10, a CAR target gene, were significantly upregulated in all Aroclor 1260-exposed groups (Fig. 2.10 A, $p<0.05$ ). In the CD groups, the fold induction of Cyp2b10 was slightly higher in the lower dose (20 $\mathrm{mg} / \mathrm{kg}$ ) as compared to the higher dose (200 mg/kg) (approximately 1000 -fold $v s$. 500 -fold respectively). Feeding a HFD markedly reduced the fold induction of Cyp2b10 with inductions of 4.3-fold and 12-fold being observed at $20 \mathrm{mg} / \mathrm{kg}$ and $200 \mathrm{mg} / \mathrm{kg}$ exposures, respectively. Thus the reduction in fold induction caused by HFD feeding vs. CD feeding at $20 \mathrm{mg} / \mathrm{kg}$ and $200 \mathrm{mg} / \mathrm{kg}$ was reduced by approximately 235 -fold and 41 -fold, respectively. These results appear consistent with previous studies $(100,101)$, and indicate that the ability of CAR to activate target genes is compromised when animals are fed with HFD.

Hepatic Cyp3a11 (PXR target gene) expression levels were also significantly induced in all Aroclor 1260-exposed groups (Fig. $2.10 \mathrm{~B}, p<0.05$ ). The Сyp3a11 fold induction caused by Aroclor 1260 exposure was significantly lower compared to Cyp2b10 (approximately 30- to 34-fold for the $20 \mathrm{mg} / \mathrm{kg}$ 
exposure and 20 - to 25 -fold for the $200 \mathrm{mg} / \mathrm{kg}$ exposure). Contrary to Cyp2b10 expression, HFD did not affect the fold inducibility of this particular gene. However, HFD feeding decreased the basal expression level of Cyp3a11 by approximately 8-fold.

The mRNA levels of Cyp1a2 (AhR target gene) were also measured in all the groups to determine Aroclor 1260 activation of the AhR. Hepatic Cyp1a2 expression was up-regulated in both dietary groups exposed to Aroclor 1260 only at $200 \mathrm{mg} / \mathrm{kg}$ (Fig. $2.10 \mathrm{C}, p<0.05$ ) but not at $20 \mathrm{mg} / \mathrm{kg}$. Feeding with a HFD had no effect on either fold inducibility or basal level expression of this gene. These results suggest that with Aroclor 1260, the levels of congeners that activate CAR/PXR are present in much higher concentrations than those that activate AhR and the receptor based-effects of Aroclor 1260 at the lower dose are likely to be mediated primarily through CAR/PXR activation.

In addition to direct targets, both CAR and PXR are capable of binding, potentially sequestering and altering the transcriptional activity of the transcription factor forkhead box O1 (FOXO1) (74). FOXO1 is an important transcription factor controlling the expression of a wide range of gluconeogenic and lipogenic genes. To examine if FOXO1 mediated gene transcription was being affected, we examined the effects of Aroclor exposure on PEPCK-1, a prototypical FOXO1 target gene and the rate limiting step in gluconeogenesis. HFD feeding did not affect PEPCK-1 mRNA levels alone but Aroclor 1260 exposure at $20 \mathrm{mg} / \mathrm{kg}$ significantly reduced it in the HFD group (Fig. 2.10 D). 
A

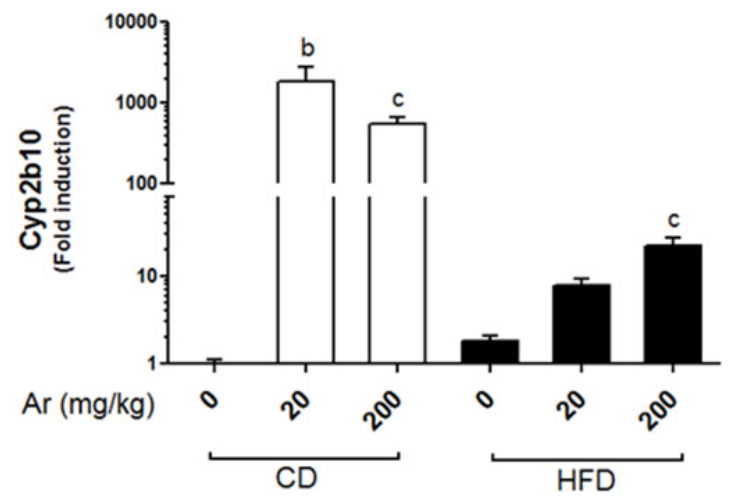

C

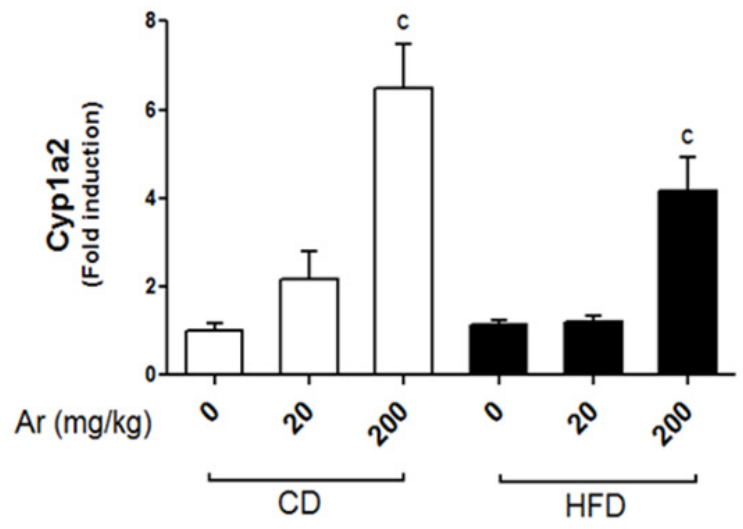

B

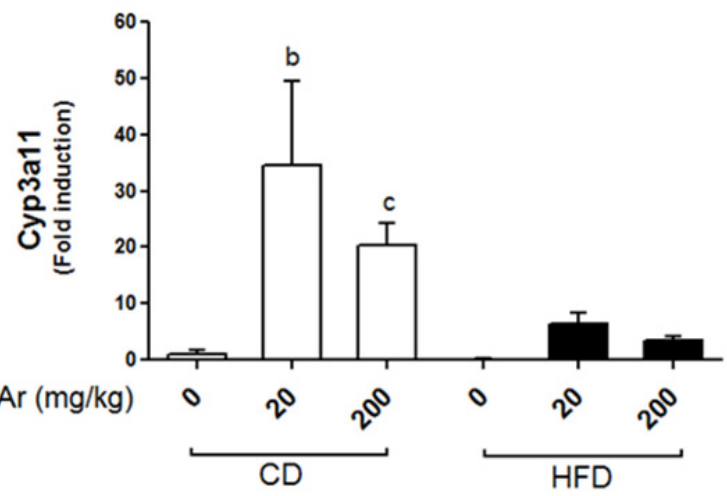

D

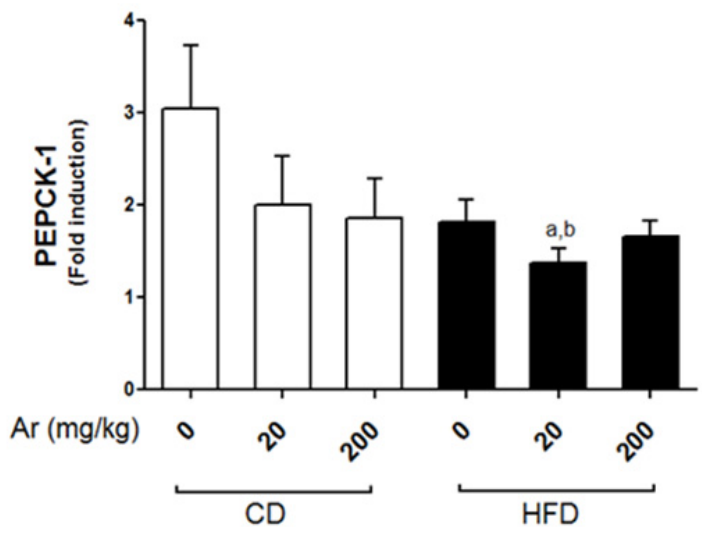


Figure 2.10. Aroclor 1260 exposure altered hepatic expression of CAR, PXR and AhR target genes.

Real-time PCR experiments showed the changes in hepatic mRNA expressions caused by Aroclor 1260 exposure for (A) Cyp2b10 (CAR target gene), (B) Cyp3a11 (PXR target gene), (C) Cyp1a2 (AhR target gene) and (D) PEPCK-1 (an indirect target of CAR and PXR). Values are mean \pm SEM, $p$ $<0.05$, a- $\Delta$ due to HFD, b- $\Delta$ due to Aroclor 1260 exposure at $20 \mathrm{mg} / \mathrm{kg}, \mathrm{c}-\Delta$ due to Aroclor 1260 exposure at $200 \mathrm{mg} / \mathrm{kg}$. CD-control diet, HFD-high fat diet, ArAroclor 1260. Figure adapted from Wahlang et al., Toxicol Appl Pharmacol, 279 (3), 2014. 


\section{DISCUSSION}

Although several PCB mixtures were commercially produced and used widely (e.g. Aroclor(s) 1260, 1254, 1248, 1242 and 1016), Aroclor 1260 was selected for this study because of the similarity in its congener composition pattern to that in human fat (36). The major congeners in Aroclor 1260 are the high molecular weight PCBs that have either 5-, 6-, 7- or 8- chlorine substituents, which in turn results in di-ortho substitution, and hence are non-coplanar in structure. These high molecular weight PCBs are not well metabolized and therefore bio-accumulate in humans (35). PCB toxicity has been associated with cancer, endocrine disruption, and impaired cognitive development, but recent epidemiologic studies have shown that PCB exposures can also result in metabolic disorders associated with NAFLD, including obesity, insulin resistance/diabetes, and the metabolic syndrome $(41,42,102)$. Occupational exposure to PCB mixtures has also been associated with elevated plasma levels of liver enzymes (18). Chronic exposures to these chlorinated compounds appear to disrupt both lipid and glucose homeostasis and consequently lead to diabetes and associated metabolic disorders. The current study investigated the effects of environmental pollutant-nutrient interactions, which is clinically relevant because all humans are exposed to PCBs and over $75 \%$ of the US adult population is considered to be either overweight or obese. Exposure to toxicants such as PCBs may act as a 'second hit' that eventually drives this population to steatohepatitis and the metabolic syndrome. 
PCBs bio-accumulate in the liver and adipose tissue due to their hydrophobicity, thus, making these sites principal targets for PCB toxicity. Lipidadjusted serum PCB levels were measured in NHANES participants and in the PCB exposed Anniston cohort with the highest reported levels ranged from 75$170 \mathrm{ng} / \mathrm{g}(18,46)$. Additionally, the National Toxicology Program (NTP) studies measured PCB levels in a 2-year gavage study in rats. Interestingly, PCB liver levels were at least 10 -fold higher and adipose levels were at least 200 -fold higher than lipid-adjusted serum levels irrespective of the dose administered or treatment time (22). Although Aroclor 1260 levels were not measured in our study, we speculate that the distribution will be similar to other PCBs used in NTP studies. In those studies, a $20 \mathrm{mg} / \mathrm{kg}$ cumulative dose yielded the following levels: serum-176 ng/g, liver-3,663 ng/g and adipose- $92,840 \mathrm{ng} / \mathrm{g}$ while a 200 $\mathrm{mg} / \mathrm{kg}$ cumulative dose yielded levels: serum-1,788 ng/g, liver-34,010 ng/g and adipose-1,118,300 $\mathrm{ng} / \mathrm{g}$. Thus the $20 \mathrm{mg} / \mathrm{kg}$ dose employed is expected to produce serum levels similar to the maximum levels reported for the Anniston cohort (170.4 ng/g). The $200 \mathrm{mg} / \mathrm{kg}$ dose is similar to that used in the NTP TR 530 for cancer studies. In the present study, we exposed mice to PCBs using gavage which was designed to mimic human exposure routes. A potential caveat is that these mice received either a single exposure or four separate exposures rather than the intermittent exposures that humans encounter from eating PCBcontaminated food. We also attempted to have several weeks between exposure to PCBs and the measurement of study endpoints to maximize the effects from bio-accumulated PCBs rather than any metabolized congeners. 
The main finding from this study was the Aroclor 1260-mediated transition of steatosis to steatohepatitis in the diet-induced obesity model. Paradoxically, $\mathrm{NASH}$ was associated with decreased \% fat composition and increased lean body mass at the low dose exposure (Fig. 2.2). Additionally, CD groups showed a decrease in body weight gain which may be attributed to stress experienced due to oral gavage. None of the CD groups exposed to Aroclor 1260 manifested hepatitis (H\&E staining). Contrarily, Aroclor 1260 exposure in HFD groups worsened liver necro-inflammation (Fig. 2.3). Co-exposure to HFD and Aroclor $1260(20 \mathrm{mg} / \mathrm{kg})$ resulted in elevated serum ALT, IL-6 and tPAI-1 and upregulated hepatic TNFa expression (Fig. 2.5). However, Aroclor 1260 at 200 $\mathrm{mg} / \mathrm{kg}$ did not induce systemic inflammation, despite histologic signs of liver injury.

The results from this study differed markedly from our earlier work on a single congener, PCB 153, where HFD+PCB 153 co-exposure worsened steatosis and obesity in male C57BI/6J mice without causing inflammation (48). We documented PCB 153-mediated adipokine dysregulation; a phenomenon which was absent in the current study. Furthermore, HFD+PCB 153 co-exposure altered hepatic expression of genes involved in fatty acid metabolism including increased FAS and decreased CPT1A mRNA expression. Although PCB 153 is present in Aroclor 1260, other congeners are present in this PCB mixture that may contribute to steatohepatitis and yet have no effect on obesity. Thus, exposing animals to a mixture can yield outcomes that are entirely different from that of a single congener. 
Hepatic P450s, including Cyp3a11 (PXR target) and Cyp2b10 (CAR target), were induced by Aroclor 1260 exposure in both CD and HFD-fed mice (Fig. 2.10). PXR and CAR are critically involved in xenobiotic metabolism and drug disposition, but recent studies demonstrated the importance of CAR and PXR regulation on physiological processes such as glucose and lipid metabolism, and this could impact NASH $(60,103,104)$. Gao et al. demonstrated CAR as an anti-obesity receptor whose activation was protective against dietinduced obesity and insulin resistance (72). On the contrary, the role of PXR in obesity remains controversial with studies reporting either anti-obesity or obesity promoting effects $(75,77,105)$. Our RT-PCR data strongly suggest CAR activation by Aroclor 1260 as indicated by the $\sim 1000$ - to 500 -fold induction of Cyp2b10 at both the low and high exposure levels. Cyp3a11 induction is a hallmark of PXR activation, but activated CAR can also bind to the Cyp3a11 response element and drive its expression, albeit, at lower levels. Although Cyp3a11 was induced by $\sim 30$-fold, this induction may be mediated by CAR rather than PXR. These results indicate that Aroclor 1260 exposure activated CAR/PXR, hence the possible obesity-protective effects seen in the HFD-fed mice exposed to Aroclor 1260.

Furthermore, CAR/PXR activation suppresses FOXO1-insulin response sequence (IRS) binding activity (Kodama et al., 2004) resulting in decreased gluconeogenesis and hence the lowered fasting blood glucose levels seen in $\mathrm{CD}+$ Aroclor 1260 groups (Fig. 2.6). Additionally, FOXO1, is a negative regulator of SREBP1 transcription and its sequestration by activated CAR/PXR could 
result in increased SREBP1 gene expression $(74,106)$. Notably in our study, hepatic SREBP-1c expression was induced in HFD-fed mice exposed to Aroclor 1260. However, activated CAR and PXR can transcriptionally activate the antilipogenic gene Insig, consequently leading to reduced SREBP1 activity and decreased SREBP1 target gene expression such as FAS (73). Hence, the decrease in FAS expression observed in this study could possibly be due to loss of SREBP1 activity even though the gene was induced. Additionally, CD36, a lipid scavenger receptor and target gene shared by AhR, PXR and LXR was also induced by Aroclor 1260 (Fig. 2.7). Other novel findings in this study included induction of CPT1A and decreased hepatic FAS expression with Aroclor 1260 exposure in HFD-fed mice. In concert, the effects of Aroclor 1260 on lipogenesis appeared complex, and interactions with CAR/PXR could contribute to the observed decrease in \% fat composition in HFD+Aroclor 1260 groups. Moreover, glucose metabolism was abnormal in HFD-fed mice exposed to Aroclor 1260 (200 mg/kg), because, while HOMA-IR was lowered, glucose tolerance failed to improve. It appears that HOMA-IR and glucose tolerance test may be insufficient to evaluate glucose metabolism in PCB studies, given the partially divergent effects of PCB exposure in the fed and fasted state. However, these observations need to be pursued further to elucidate the mechanisms involved.

Regardless of Aroclor 1260 exposure, it appeared that HFD consumption reduced the induction of CAR/PXR target genes as compared to CD consumption and it was only HFD-fed mice exposed to Aroclor 1260 that exhibited liver injury. Therefore, it is plausible to say that activation of xenobiotic 
receptors such as CAR/PXR protect against PCB toxicity in a low fat diet setting. Therefore HFD consumption interferes with CAR/PXR activation by PCBs, and therefore attenuates the protective effects of these receptors against PCB toxicity with the net result being increased liver injury only in Aroclor 1260+HFD coexposed animals. The progression of NAFLD from simple steatosis to steatohepatitis requires both hepatic fat accumulation and inflammation. In this study, it was observed that in steatotic mice exposed to low dose Aroclor 1260, inflammation and liver injury was aggravated, while at the high dose, inflammation was suppressed and liver injury was attenuated. Our results suggest that it is the inflammatory dysfunction that PCBs induce rather than the degree of steatosis observed that may dictate appearance of steatohepatitis.

Hepatic expression of Cyp1a2 (AhR target gene) was induced only in groups receiving Aroclor 1260 at the highest dose tested (Fig. 2.10), suggesting dose-dependent activation of this receptor. AhR activation by PCBs is well documented with coplanar ('dioxin-like') PCBs including PCB 126 being good rodent AhR activators. A wasting syndrome and chloracne are characteristic features of AhR activation by its classic ligand TCDD (107). AhR activation is also associated with immune suppression via AhR interference with NF-KB signaling (108). Consistent with these results, animals exposed to Aroclor 1260 (200 mg/kg) displayed lower body weight and a suppression of serum proinflammatory cytokines and resistin levels (Fig. 2.5). Resistin, also known as the adipocyte secretory factor, is secreted by the adipose tissue and appears to participate in inflammatory processes as well (109). While reduced body weight 
has frequently been reported with animals exposed to AhR ligands, in murine and human models, steatosis increases, presumably due to a redistribution of dietary fat (110). Thus activation of the AhR may lead to increased steatosis but decreased steatohepatitis, as a function of its immunosuppressive effects. The activation AhR by Aroclor 1260 is likely due to the presence of coplanar congeners such as PCB 126 but these compounds exist in relatively low percentages in this mixture $(<1 \%)$, and hence a higher Aroclor 1260 exposure level is required to observe the 'dioxin-like' effects. Therefore, absence of inflammation at the higher dose may be due to the immune-suppressive properties of activated AhR.

Thus, we identified both CAR/PXR and AhR activation as potential mode(s) of action of this PCB mixture in NASH. Nonetheless, rodent and human receptors may have differences pertaining to ligand binding activity and target gene battery. Off target effects are also possible mechanisms in PCB-driven $\mathrm{NASH}$, but these were not evaluated in this study. Moreover, the current study failed to distinguish between CAR and PXR activation which is a potential drawback since the observed Aroclor 1260 effects may be based solely on CAR activation. Thus further investigation to distinguish between the CAR an $d$ PXRmediated effects using PXR/CAR knockout models is required. The study also failed to assess overall metabolism and employing metabolic chambers would have been useful in this regard. Furthermore, another drawback in this study was using serum ALT/AST as a NASH biomarker based on low sensitivity, and evaluating other biomarkers is a possibility in future studies (50). In addition, our 
studies were performed using male mice; hence it is pertinent to note that the observed effects may vary with gender and species.

In conclusion, Aroclor 1260 exposure caused toxicant-associated steatohepatitis in animals fed with HFD. In contrast to our previous study wherein a single congener (PCB 153) was used, this PCB mixture neither increased the body weight/visceral adiposity nor worsened insulin resistance/diet-induced obesity. There was a significant difference between the low and high exposure doses in terms of hepatic/systemic inflammation, which could potentially be due to AhR activation. Our additional findings demonstrate that Aroclor 1260 activated CAR and PXR and to a lesser extent AhR, suggesting congener composition and exposure levels to be critical in determining a mixture's mode(s) of actions. Lastly, CAR and PXR activation could be protective against PCBmediated toxicity but HFD consumption may blunt this protection. More studies are needed on the role of PCB-nuclear receptor interactions in steatohepatitis. 


\section{CHAPTER 3}

\section{EVALUATING THE ROLE OF CAR AND PXR IN AROCLOR 1260-INDUCED LIVER INJURY \\ INTRODUCTION}

Our initial studies in a diet-induced obesity mouse model demonstrated that Aroclor 1260 exposure had modest effects on a control diet but induced steatohepatitis when animals were fed with a high fat diet. In animals fed a high fat diet, Aroclor 1260 exposure did not exacerbate obesity. However, in the liver, Aroclor 1260 exposure $(20 \mathrm{mg} / \mathrm{kg})$ activated nuclear receptors, including CAR and PXR as the cytochrome P450s, namely, Cyp2b10 and Cyp3a11 were robustly induced. At higher Aroclor 1260 exposures (200 mg/kg), the AhR was also activated. Furthermore, PCB activation of the AhR has been well studied and its activation has been linked to hepatic cancer. However PCB activation of CAR and PXR and the subsequent contribution of these activated receptors to PCB effects and toxicity have not been thoroughly evaluated, especially in terms of obesity and NAFLD/NASH.

In this study, the role of CAR and PXR in Aroclor 1260-induced liver injury was further characterized. We hypothesized that CAR and PXR play a role in the transition of steatosis to steatohepatitis caused by Aroclor 1260 exposure. The CAR and PXR knockout mouse models were utilized to demonstrate that both 
receptors were required for normal physiology and that they both played a protective role in PCB+HFD-induced liver disease. Additionally, in the previous study, mice receiving Aroclor 1260 at $20 \mathrm{mg} / \mathrm{kg}$ elicited CAR and PXR activation as well as $\mathrm{NASH}$; therefore this dose was employed again in the present study. 


\section{MATERIALS AND METHODS}

\section{Animals and diets}

The animal protocol was approved by the University of Louisville Institutional Animal Care and Use Committee. Wild type male C57BI/6J mice (WT, 8 weeks old; The Jackson Laboratory, Bar Harbor, ME, USA) and CAR ${ }^{-1-}$ and $P X R^{-/-}$mice (Taconic, Hudson, NY) were divided into 6 study groups $(n=10)$ based on Aroclor 1260 exposure utilizing a $2 \times 3$ design. All mice were fed a high fat diet (HFD, 42\% kCal from fat; TD.88137 Harlan Teklad) in this 12 week study. On Week 1, Aroclor 1260 (AccuStandard, CT, USA) was administered in corn oil by oral gavage (vs. corn oil alone) at $20 \mathrm{mg} / \mathrm{kg}$. This dose was designed to mimic the maximum human PCB exposures seen in the PCB-exposed Anniston cohort. Mice were housed in a temperature- and light controlled-room (12 hour light; 12 hour dark) with food and water ad libitum. During Week 8-9, mice were placed in metabolic chambers (PhenoMaster, TSE systems, Chesterfield, MO) overnight to assess food/drink consumption and physical activity. A glucose tolerance test was performed at Week 11, and the animals were euthanized (ketamine/ xylazine, $100 / 20 \mathrm{mg} / \mathrm{kg}$ body weight, i.p.) at the end of Week 12. Prior to euthanasia, the animals were analyzed for body fat composition by dual energy X-ray absorptiometry (DEXA) scanning (Lunar PIXImus densitometer, WI, USA).

Thus six different groups were evaluated; WT, WT+Aroclor $1260, \mathrm{CAR}^{-1}, \mathrm{CAR}^{-/-}$ +Aroclor 1260, $\mathrm{PXR}^{-/-}, \mathrm{PXR}^{-1-}+$ Aroclor 1260.

\section{Glucose tolerance test}

See detailed method in Chapter 2. 


\section{Histological studies}

See detailed method in Chapter 2.

\section{Cytokine and adipokine measurement}

See detailed method in Chapter 2.

\section{Measurement of hepatic triglyceride and cholesterol content}

See detailed method in Chapter 2.

\section{Real-time PCR}

See detailed method in Chapter 2. Additional primer sequences from Taqman Gene Expression Assays (Applied Biosystems, Foster City, CA) that were utilized in the current study included UDP glucuronosyltransferase 1 family, polypeptide A1 (Ugt1a1); (Mm02603337_m1), patatin-like phospholipase domain containing 2 (PNPLA2); (Mm00503040_m1), PXR (Mm01344139_m1), CAR (Mm01283978_m1), fatty acid binding protein-1 (FABP1); (Mm00444340_m1), glucokinase (Gck); (Mm00439129_m1), GLUT-2, (Mm00446229_m1), GLUT-4 (Mm01245502_m1), glucose-6-phosphatase (G6Pase); (Mm00839363_m1), Insig-1 (Mm00463389_m1) and Insig-2 (Mm01308255_m1).

\section{Immunoblots}

Frozen liver samples $(0.1 \mathrm{~g})$ were homogenized in $0.5 \mathrm{~mL}$ radioimmunoprecipitation assay (RIPA) buffer (20 mM Tris, pH 7.4, $150 \mathrm{mM} \mathrm{NaCl}, 1$ mM EDTA, 1 mM EGTA, $1 \mathrm{mM} \beta$-glycerophosphate, $1 \mathrm{mM}$ sodium vanadate, and $1 \% \mathrm{w} / \mathrm{w}$ Triton $\mathrm{X}-100 \mathrm{w} / \mathrm{v}$ ) containing $1 \mathrm{mM}$ phenylmethylsulphonyl fluoride, protease and phosphatase (tyrosine and serine/threonine) inhibitor cocktails (Sigma Aldrich, St. Louis, MO, USA). Lysates were sonicated at $4{ }^{\circ} \mathrm{C}$ for $4 \mathrm{~h}$ and 
subsequently centrifuged for 5 minutes at $16,000 \mathrm{~g}$. The protein concentration of the supernatants was determined using the Bicinchoninic Acid Protein Assay Kit (Sigma Aldrich). Total protein was diluted in RIPA buffer and mixed with sample loading buffer [250 mM Tris, pH 7.4, 10\% sodium dodecyl sulfate (SDS), $20 \% \beta$ mercaptoethanol $\mathrm{w} / \mathrm{v}, 40 \%$ glycerol, and $0.05 \%$ bromophenol blue] and incubated at $95^{\circ} \mathrm{C}$ for 5 minutes. The samples were loaded onto SDS-polyacrylamide gels (Bio-Rad Laboratories, Hercules, CA, USA), followed by electrophoresis and Western blotting onto polyvinylidene difluoridemembranes (Immobilon-P; Millipore Corp, Billerica, MA, USA). Antibodies against the sterol regulatory element binding protein 1 (SREBP1, Abcam, Cambridge, MA, USA), AMPactivated protein kinase a (AMPKa, Cell Signaling Technology, Danvers, MA, USA), phospho-AMPKa (p-AMPKa, Cell Signaling Technology), mammalian target of rapamycin (mTOR, Cell Signaling Technology), phospho-mTOR ( $p$ mTOR, Cell Signaling Technology) and $\beta$-actin (Santa Cruz Biotechnology, Dallas, TX, USA) were used at dilutions recommended by the suppliers. Horseradish peroxidase-coupled secondary antibodies were obtained from Abcam and Cell Signaling Technology. Chemiluminescence detection was performed using the Pierce ECL2 western blotting substrate reagents (Thermo Scientific, Wilmington, DE, USA). Densitometric quantitation was performed with the Image Lab software (Bio-Rad Laboratories).

\section{Statistical Analysis}

Statistical analyses were performed using SigmaPlot version 11.0 (Systat Software, Inc., San Hose, CA, USA). Data are expressed as mean \pm SEM. 
Multiple group data were compared using Two Way ANOVA followed by Tukey Test for post-hoc all pairwise comparisons. $P<0.05$ was considered statistically significant. 


\section{RESULTS}

\section{Effects of CAR and PXR on bodyweight and adiposity}

Bodyweight was measured weekly throughout the 12-week study (Fig. 3.1 A). All groups experienced bodyweight gain until Week 11. The bodyweight drop was possibly due to stress caused by the glucose tolerance test that was performed on Week 11. Aroclor 1260 exposure had no significant effect on the percent (\%) increase in bodyweight gain calculated in the WT or $\mathrm{PXR}^{-/-}$groups (Fig. 3.1 B). However, the $\mathrm{CAR}^{-/-}$mice exposed to Aroclor 1260 showed a lower $\%$ increase in the bodyweight gain than any other group. The \% body fat composition was evaluated by DEXA scanning prior to harvesting the tissues (Fig. 3.1 C). Because all groups were on HFD feeding, the average \% body fat composition among the animals was $\sim 40 \%$. However, the $\mathrm{PXR}^{-/}$groups displayed significantly higher \% body fat composition with or without Aroclor 1260 exposure. The PXR ${ }^{-/}$groups also showed significantly lower lean body mass (g), irrespective of Aroclor 1260 exposure (Fig. 3.1 D). The liver to bodyweight ratio (LW/BW) was calculated and the $\mathrm{PXR}^{-/-}$groups showed significantly higher liver mass and LW/BW when compared to any other group (Fig. 3.1 E).

The epididymal fat sections were stained with H\&E (Fig. 3.2 A) and the adipocyte size was measured using Image J software (Fig. 3.2 B). The mean adipocyte size $\left(\mu \mathrm{m}^{2}\right)$ was lower in the $\mathrm{CAR}^{-/-}$groups irrespective of Aroclor 1260 exposure. There was no difference in the adipocyte size in the WT or $\mathrm{PXR}^{-/-}$mice irrespective of Aroclor 1260 exposure. Ad libitum food consumption per mouse per day (g) was calculated over the 12-week period of the study (Fig. 3.2 C). 
There was a drop in the food consumption rate on Week 2 for all groups possibly due to the corn oil gavage. During the study period, the $\mathrm{CAR}^{-{ }_{-}}$and $\mathrm{PXR}^{-/-}$mice exposed to Aroclor 1260 showed relatively lower food consumption rate while the WT groups showed higher food consumption rate with or without Aroclor 1260 exposure. 
A

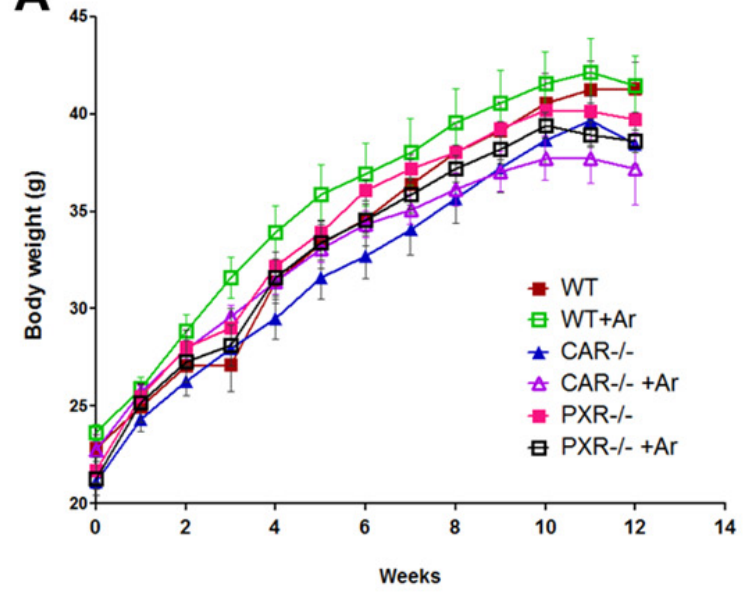

C

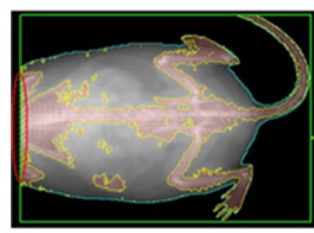

$\underline{W T}+\operatorname{Ar} 1260$

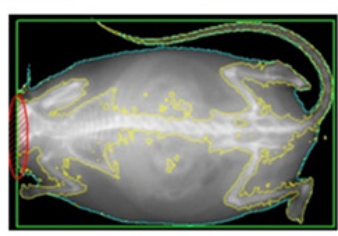

$\underline{\text { PXR }}^{-}+\operatorname{Ar} 1260$

D

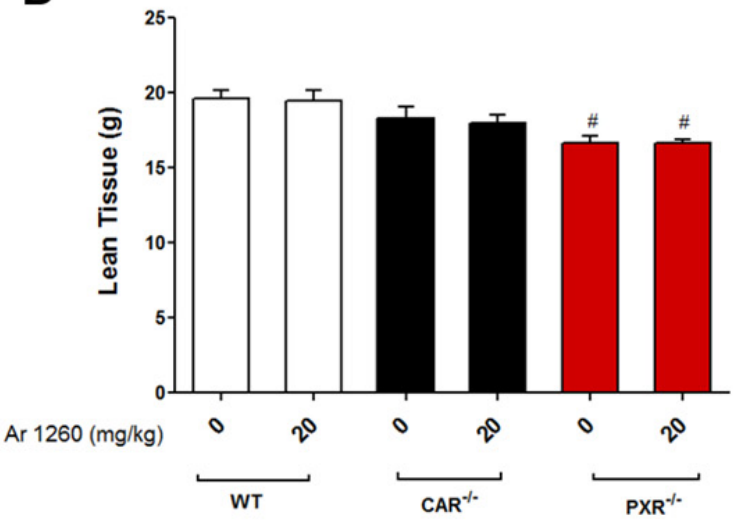

B
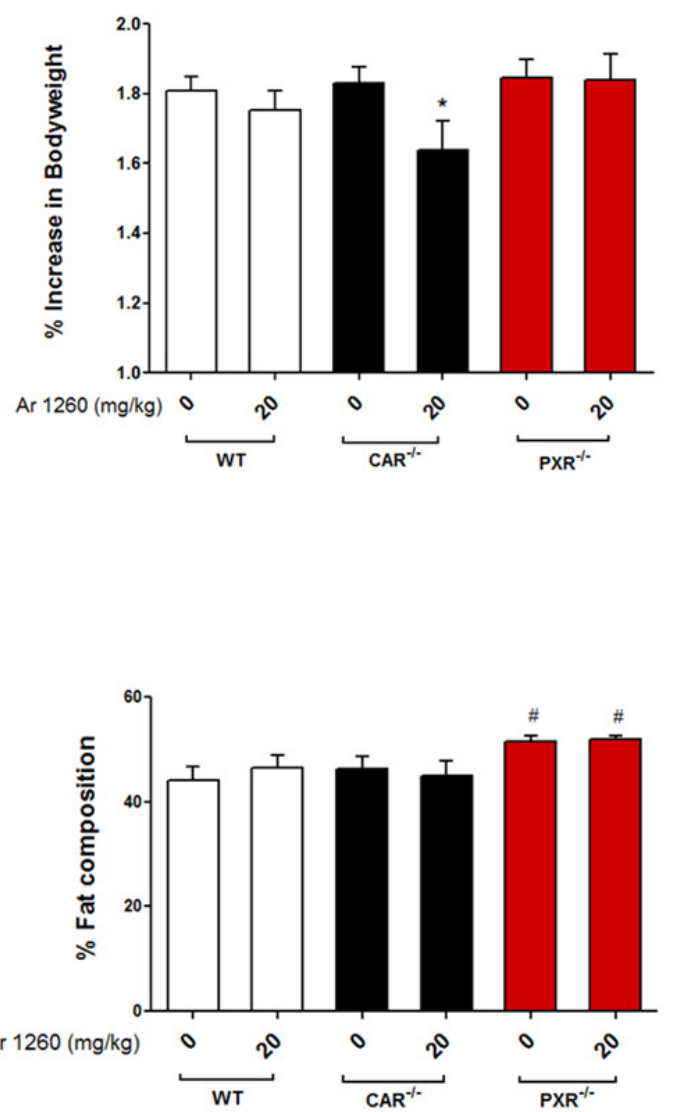

E

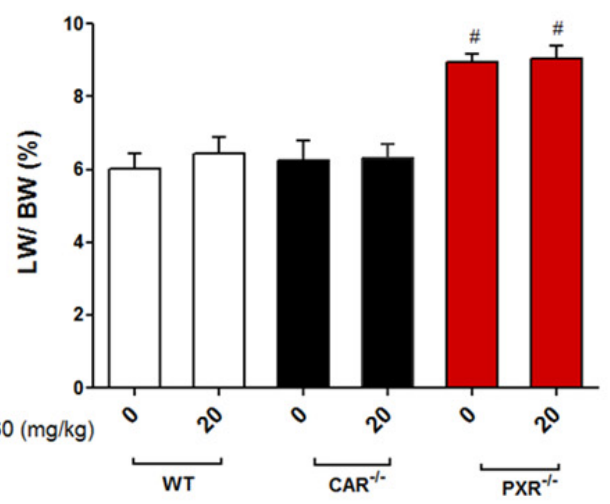


Figure 3.1. Effects of Aroclor 1260 exposure on body weight and visceral adiposity in $\mathrm{CAR}^{-/-}$and $\mathrm{PXR}^{-/-}$mice.

(A) Increase in body weight with time for C57BL/6 (WT), CAR ${ }^{-/-}$and $\mathrm{PXR}^{-/-}$ mice $(n=10)$ fed with a $42 \%$ milk fat diet. Body weight measurements were taken weekly from Week 1 to Week 12. (B) The \% increase in body weight gain with time was calculated and the body weight at Week 1 was taken as $100 \%$. (C) \% fat composition and (D) lean tissue mass (g) were measured using the DEXA scanner. PXR knockout mice demonstrated a higher \% fat composition and lowered lean tissue mass vs. WT and $\mathrm{CAR}^{-/}$groups. (E) Livers were removed and weighed at euthanasia and the liver to bodyweight ratio was calculated. Values are mean \pm SEM, $p<0.05,{ }^{*}-\Delta$ due to Aroclor 1260 exposure, \# - $\Delta$ due to knocking out CAR or PXR. 
A

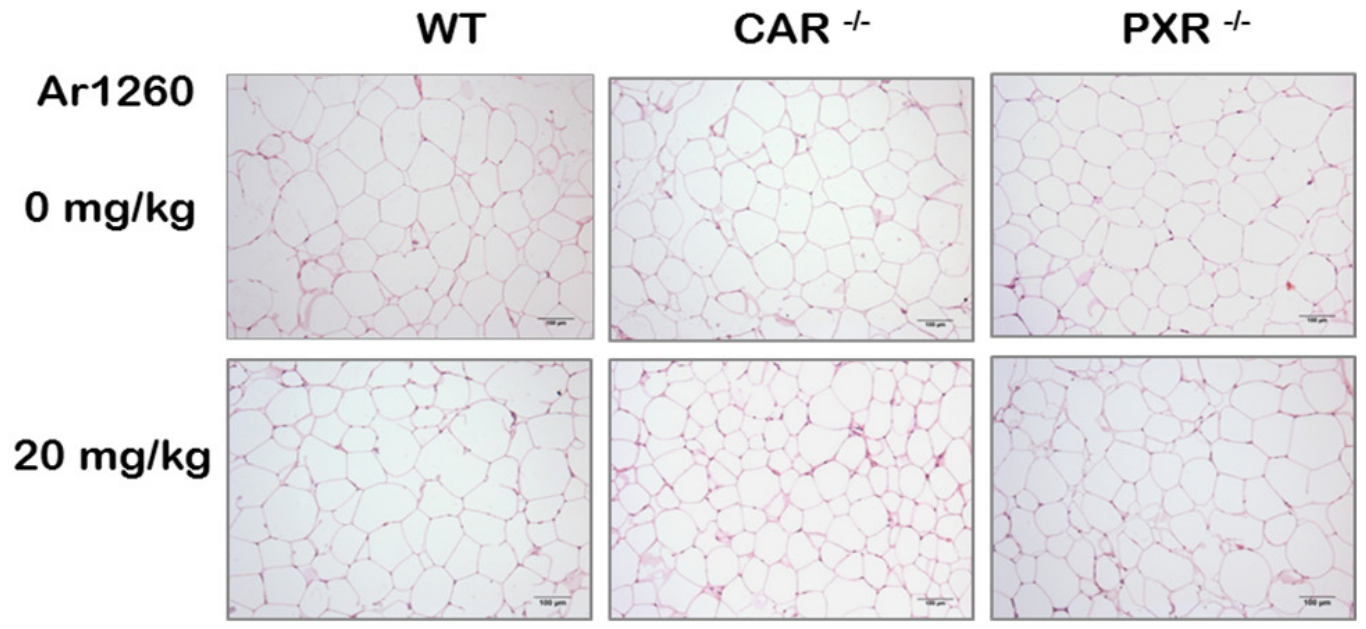

B

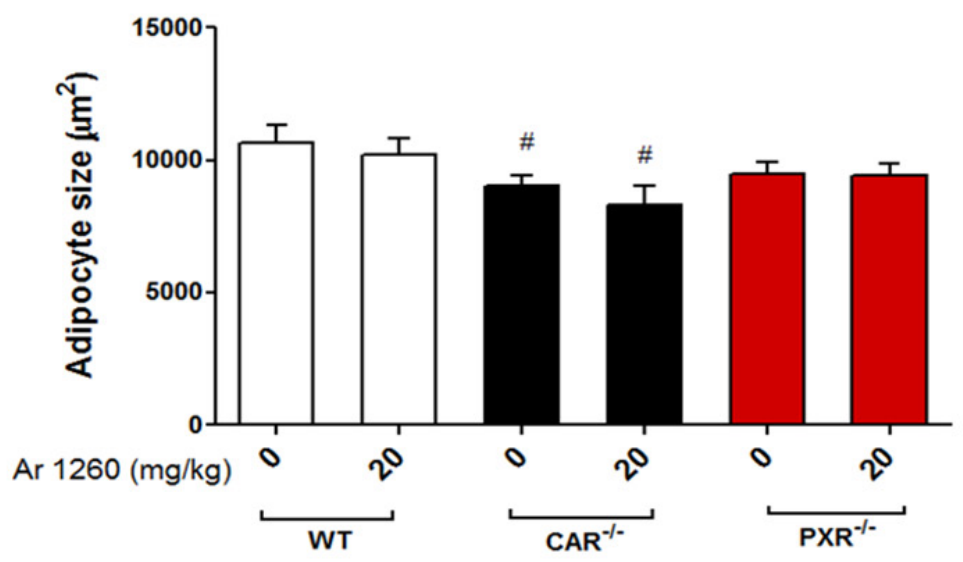

C

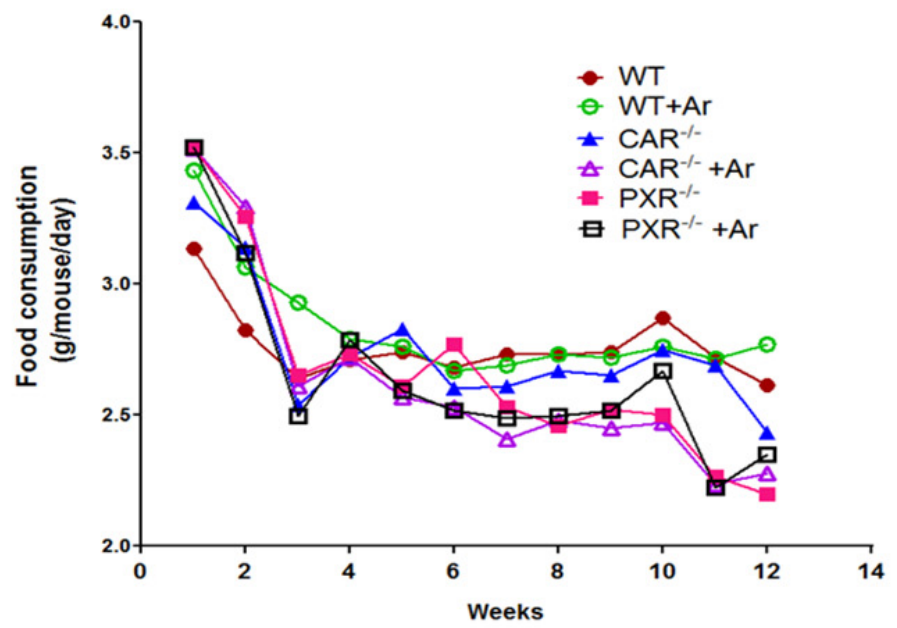


Figure 3.2. Effects of Aroclor 1260 exposure on the adipocyte size and food consumption in $\mathrm{CAR}^{-/-}$and $\mathrm{PXR}^{-/-}$mice.

(A) The epididymal adipose tissue was stained with H\&E. (B) Adipocyte size $\left(\mu \mathrm{m}^{2}\right)$ was measured and average cell size of $>100$ cells for each group was calculated. (C) Food consumption per mouse per day (g) was measured throughout the 12 week period. Values are mean \pm SEM, $p<0.05,{ }^{*}-\Delta$ due to Aroclor 1260 exposure, \# - $\Delta$ due to knocking out CAR or PXR. 


\section{Metabolic chamber studies}

Animals were placed in metabolic cages at the beginning of Week 8 for metabolic assessment. Oxygen consumption $\left(\mathrm{vO}_{2}\right)$ and carbon dioxide production $\left(\mathrm{vCO}_{2}\right)$ were monitored and the respiration exchange rate (RER, $\mathrm{vCO}_{2} / \mathrm{vO}_{2}$ ) was calculated. The measured ratio (RER) was used to estimate the respiratory quotient which indicates whether the fuel source/energy expenditure is from carbohydrate or lipid metabolism. An RER of 0.70 suggests that fat is the predominant fuel source (oxidation of a molecule of fatty acid: $23 \mathrm{O}_{2}+\mathrm{C}_{16} \mathrm{H}_{32} \mathrm{O}_{2}$

$\rightarrow 16 \mathrm{CO}_{2}+16 \mathrm{H}_{2} \mathrm{O}+129$ ATP, $\left.\mathrm{RER}=\mathrm{vCO}_{2} / \mathrm{vO}_{2}=16 \mathrm{CO}_{2} / 23 \mathrm{O}_{2}=0.7\right)$, whereas an RER of 0.85 indicates a mix of fat and carbohydrates. An RER of 1.00 or above is indicative of carbohydrate being the predominant fuel source (oxidation of a molecule of carbohydrate: $6 \mathrm{O}_{2}+\mathrm{C}_{6} \mathrm{H}_{12} \mathrm{O}_{6} \rightarrow 6 \mathrm{CO}_{2}+6 \mathrm{H}_{2} \mathrm{O}+38$ ATP, $\left.\mathrm{RER}=\mathrm{vCO}_{2} / \mathrm{vO}_{2}=6 \mathrm{CO}_{2} / 6 \mathrm{O}_{2}=1.0\right)$ and this usually occurs during intense physical activity such as exercise.

The knockout groups that were unexposed to Aroclor 1260 showed a lower RER ( 0.70), indicating a lipid-driven energy breakdown (Fig. 3.3 A). The RER was increased with Aroclor 1260 exposure in the knockout groups in both the light and dark cycle, indicating a mix of fat and carbohydrate fuel source. There was no difference in the RER in WT mice with or without Aroclor 1260 exposure. The total energy expenditure (EE) was computed using the following modified Weir equation: $E E=(3.815+1.232 \times$ RER $) \times$ VO2 (111). There were no differences in the EE between the groups (Fig. 3.3 B). 
Movement/physical activity was also assessed using the metabolic chambers. The CAR and PXR knockout mice exposed to Aroclor 1260 showed increased movement/physical activity during the light cycle relative to the unexposed knockout mice (Fig. 3.4 A). Furthermore, the $\mathrm{CAR}^{-/-}$group exposed to Aroclor 1260 demonstrated significantly lower food and drink consumption in the dark cycle vs. any other group (Fig. 3.4 B\&C). 
A
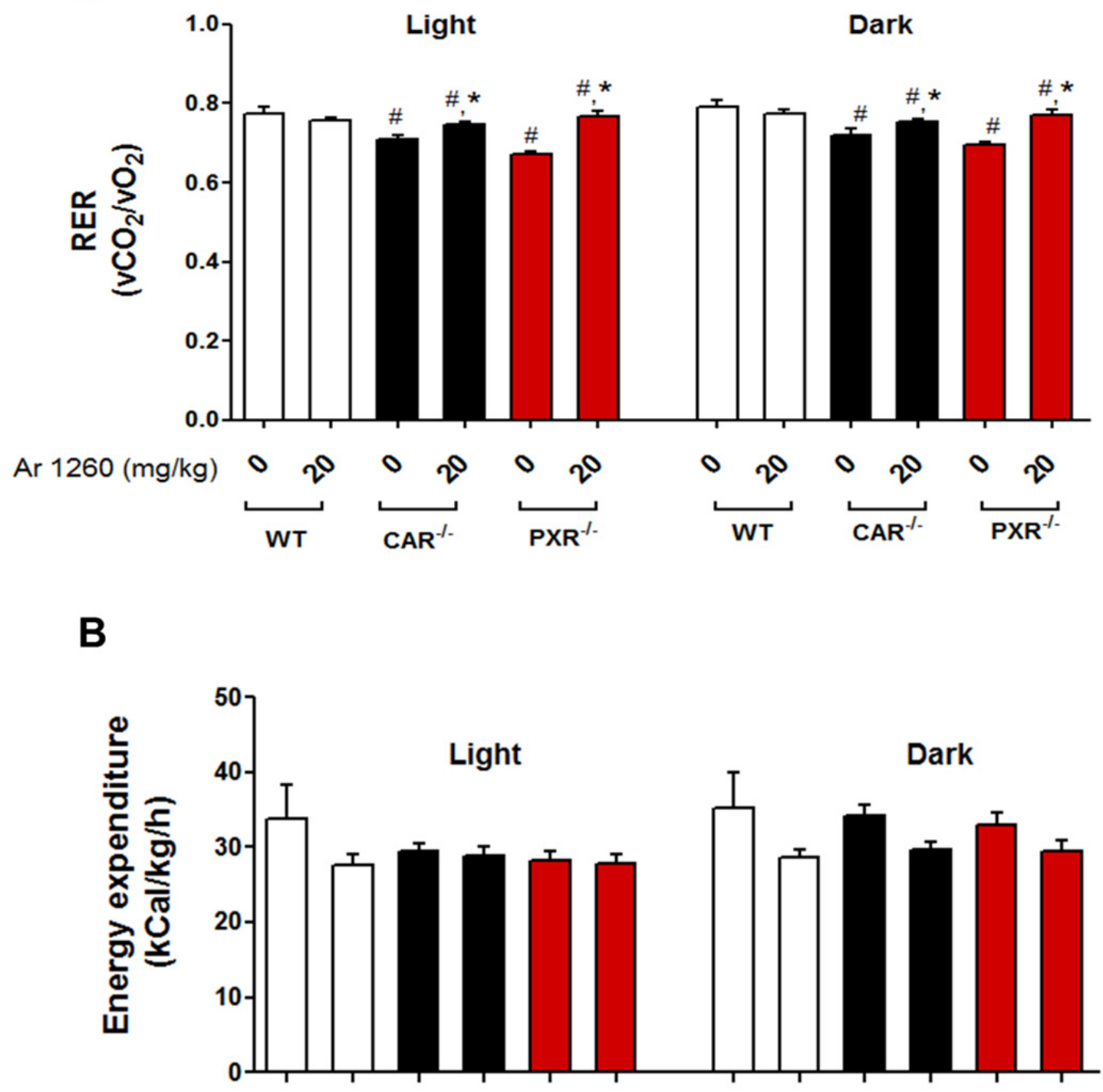

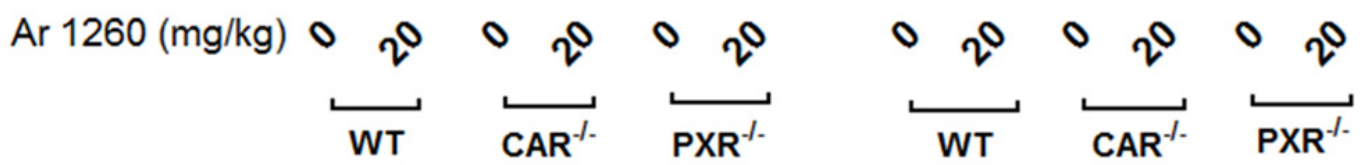


Figure 3.3. Assessment of respiration exchange rate and energy expenditure utilizing metabolic cages.

Four mice from each group were taken randomly and placed in metabolic cages for $24 \mathrm{~h}$ (12 h light and $12 \mathrm{~h}$ dark cycle). (A) The respiration exchange rate (RER) which is the ratio of $\mathrm{CO}_{2}$ exhaled to $\mathrm{O}_{2}$ consumed was calculated as an indicator of energy expenditure. (B) The total energy expenditure (EE, $\mathrm{kCal} / \mathrm{kg} / \mathrm{h})$ was calculated using the values obtained from RER and $\mathrm{VO}_{2}$. Values are mean \pm SEM, $p<0.05,{ }^{*}-\Delta$ due to Aroclor 1260 exposure, $\#-\Delta$ due to knocking out CAR or PXR. 

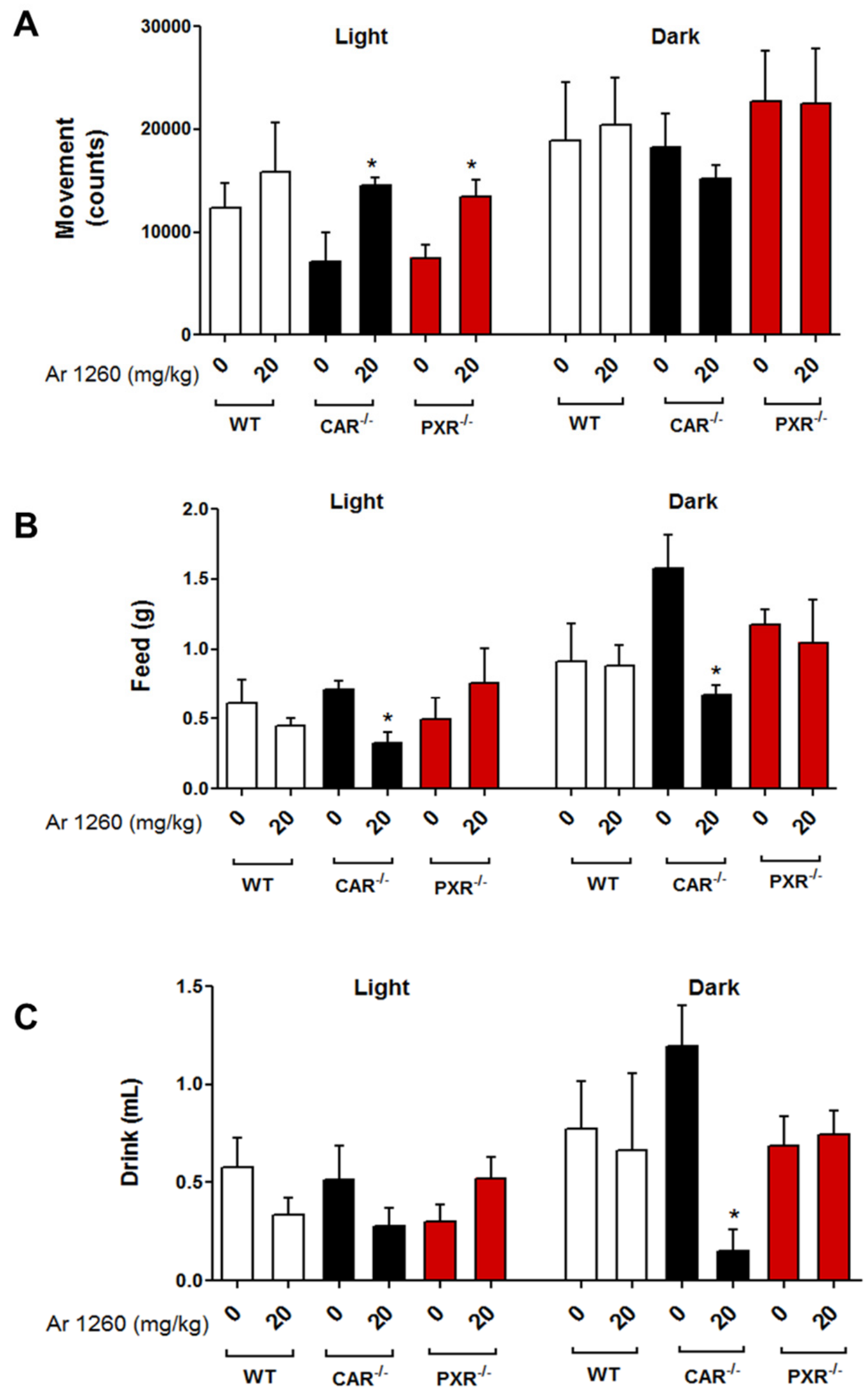
Figure 3.4. Assessment of movement, food and drink consumption utilizing metabolic cages.

Four mice from each group were taken randomly and placed in metabolic cages for $24 \mathrm{~h}$ (12 $\mathrm{h}$ light and $12 \mathrm{~h}$ dark cycle). (A) Physical activity was measured using infrared beams and sensors. The total movement (counts) which is the sum of ambulatory movement (mouse crosses two adjacent beams) and fine movement (mouse crosses the same beam twice) was calculated. The average amount of $(B)$ food $(\mathrm{g}) /$ day and $(\mathrm{C})$ water $(\mathrm{mL}) /$ day consumed per group was measured. Values are mean $\pm \mathrm{SEM}, p<0.05,{ }^{*}-\Delta$ due to Aroclor 1260 exposure, \# - $\Delta$ due to knocking out CAR or PXR, Ar-Aroclor 1260. 
The role of CAR and PXR in Aroclor 1260-induced liver injury and steatosis

All animal groups developed steatosis by the end of the study due to high fat feeding. Histological examination of liver sections demonstrated that Aroclor 1260 exposure induced liver injury in all groups of mice and this injury was independent of the presence of CAR or PXR (Fig. 3.5 A). Scattered inflammatory foci and neutrophil infiltration were also observed with CAE staining and these foci were more pronounced in the $\mathrm{PXR}^{-/-}$mice exposed to Aroclor 1260 (Fig. 3.5 B). Although exposure to Aroclor 1260 caused liver injury (H\&E and CAE staining), serum ALT was not significantly elevated (Fig. 3.5 C). Furthermore, serum AST was unaffected in all groups (Fig. 3.5 D).

Hepatic levels of triglycerides and cholesterol were quantified to measure the degree of steatosis and there were no significant differences in hepatic lipids among the groups (Fig. 3.5 E\&F). This was somewhat surprising as the $\mathrm{PXR}^{-1-}$ mice had a higher body fat composition and higher liver to body weight ratios. 

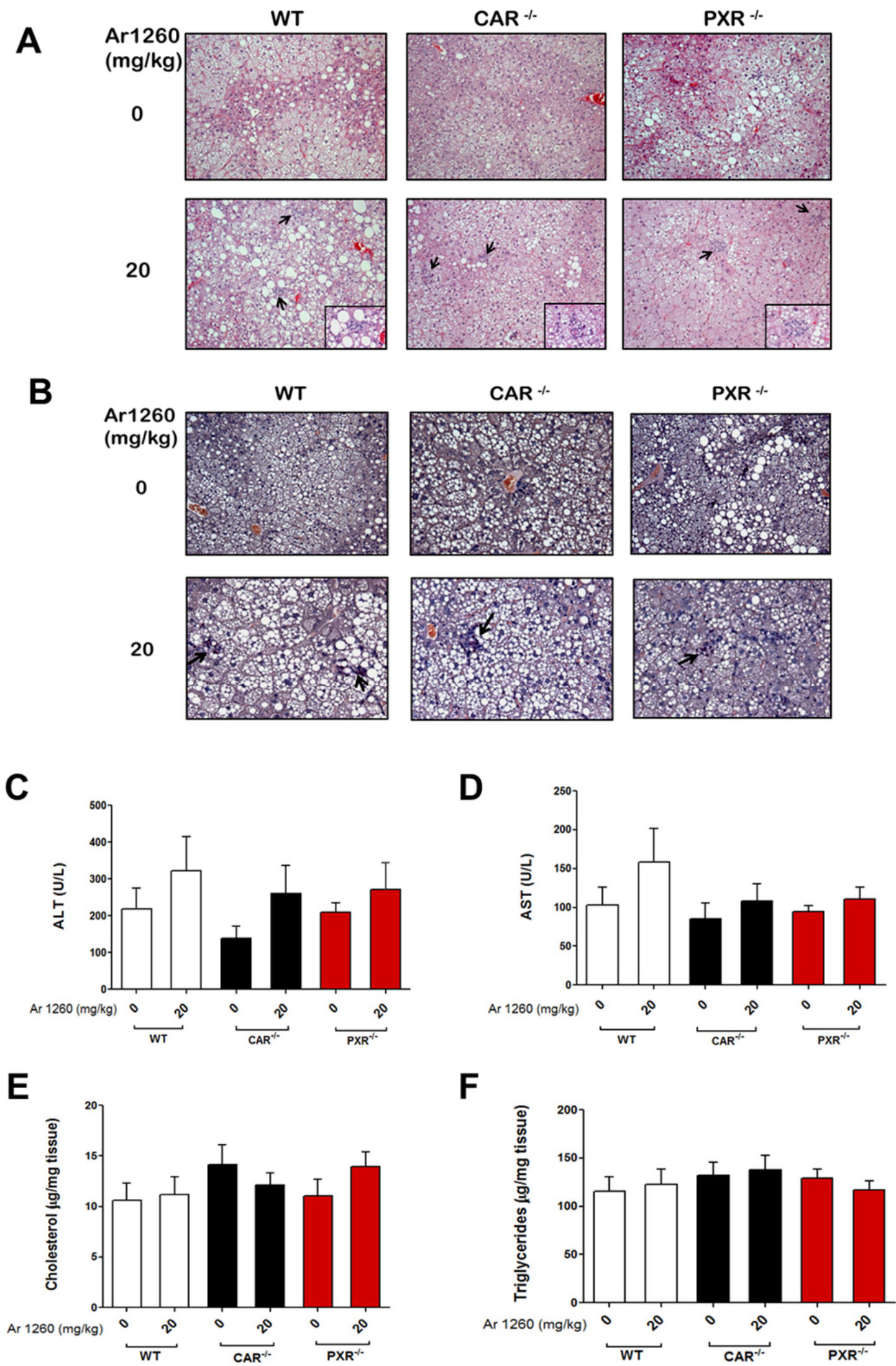

F

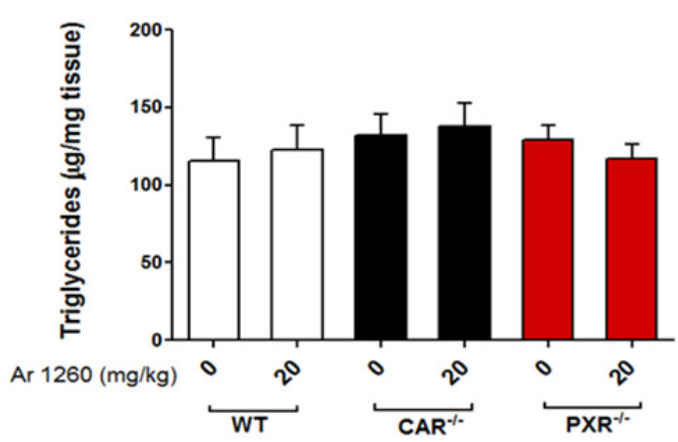


Figure 3. 5. Aroclor 1260 exposure caused steatohepatitis in WT, $\mathrm{CAR}^{-/-}$and $\mathrm{PXR}^{-/-}$mice.

(A) H\&E staining of hepatic sections established the occurrence of centrilobular hepatocellular hypertrophy, karyomegaly, and multinucleate hepatocytes in the Aroclor 1260-exposed groups. High fat diet consumption resulted in variable, centrilobular, microvesicular lipidosis while mice exposed to Aroclor 1260 exhibited occasional, small areas of necrosis and inflammation (steatohepatitis). (B) CAE staining demonstrated neutrophil infiltration in the Aroclor 1260-exposed groups. (C). Serum ALT and (D) AST levels (U/L) were measured $(n=10)$ using the Piccolo Xpress chemical analyzer. Hepatic levels of $(E)$ cholesterol and $(F)$ triglycerides were quantified $(\mu \mathrm{g} / \mathrm{mg}$ tissue $)$ in mice $(n=10)$ fed with HFD with or without Aroclor 1260 co-exposure. Values are mean \pm SEM, $p<0.05,{ }^{*}-\Delta$ due to Aroclor 1260 exposure, $\#-\Delta$ due to knocking out CAR or PXR, Ar-Aroclor 1260. 


\section{CAR and PXR are protective against Aroclor 1260-induced hepatic and systemic inflammation}

To further assess the role of nuclear receptors in Aroclor 1260-induced steatohepatitis, hepatic expression of toll like receptor 4 (TLR-4) target genes including tumor necrosis factor alpha (TNFa), interleukin 6 (IL-6), monocyte chemo-attractant protein 2 (MCP2) and monocyte inducible factor 1 alpha (MIP1a) were measured using RT-PCR. Aroclor 1260 exposure resulted in increased TNFa expression in the liver of WT mice (Fig. 3.6 A). Interestingly, the basal expression of TNFa in the knockout mice was higher than in the WT mice, regardless of Aroclor 1260 exposure. The $\mathrm{CAR}^{-/-}$mice with or without Aroclor 1260 exposure showed significantly higher TNFa mRNA levels when compared to any other group. Likewise, the basal expression of hepatic IL-6 was also higher in the knockout groups than the WT group (Fig. 3.6 B). PXR ${ }^{-1-}$ mice exposed to Aroclor 1260 showed the highest hepatic IL-6 mRNA levels among all groups. Hepatic expression of MCP2 and MIP1 1 were not significantly different between the groups (Fig. 3.6 C\&D). Overall, it appeared that knocking out CAR and PXR increased hepatic TNF $\alpha$ and IL-6 expression, irrespective of Aroclor 1260 exposure.

Serum cytokines, namely, TNFa, IL-2, interferon gamma (IFNY), IL-17, MCP1 and MIP-1a were measured using the Luminex IS system. Similar to hepatic TNFa expression, serum TNFa levels were higher in Aroclor 1260exposed WT-mice compared to unexposed mice in the WT group (Fig. 3.7). However, there was no significant difference in serum TNFa levels in the Aroclor 
1260-exposed compared to unexposed mice in the knockout groups. Likewise, serum IL-2 and IFNy were also increased in the Aroclor 1260-exposed when compared to unexposed mice in the WT group but there was no difference observed in the knockout groups. Notably, the basal levels of serum TNF $\alpha$ and IL-2 were higher in the $\mathrm{PXR}^{-/}$group, irrespective of Aroclor 1260 exposure. There was no significant increase in serum IL-17, MIP1 $\alpha$ and MCP1 among the groups. 

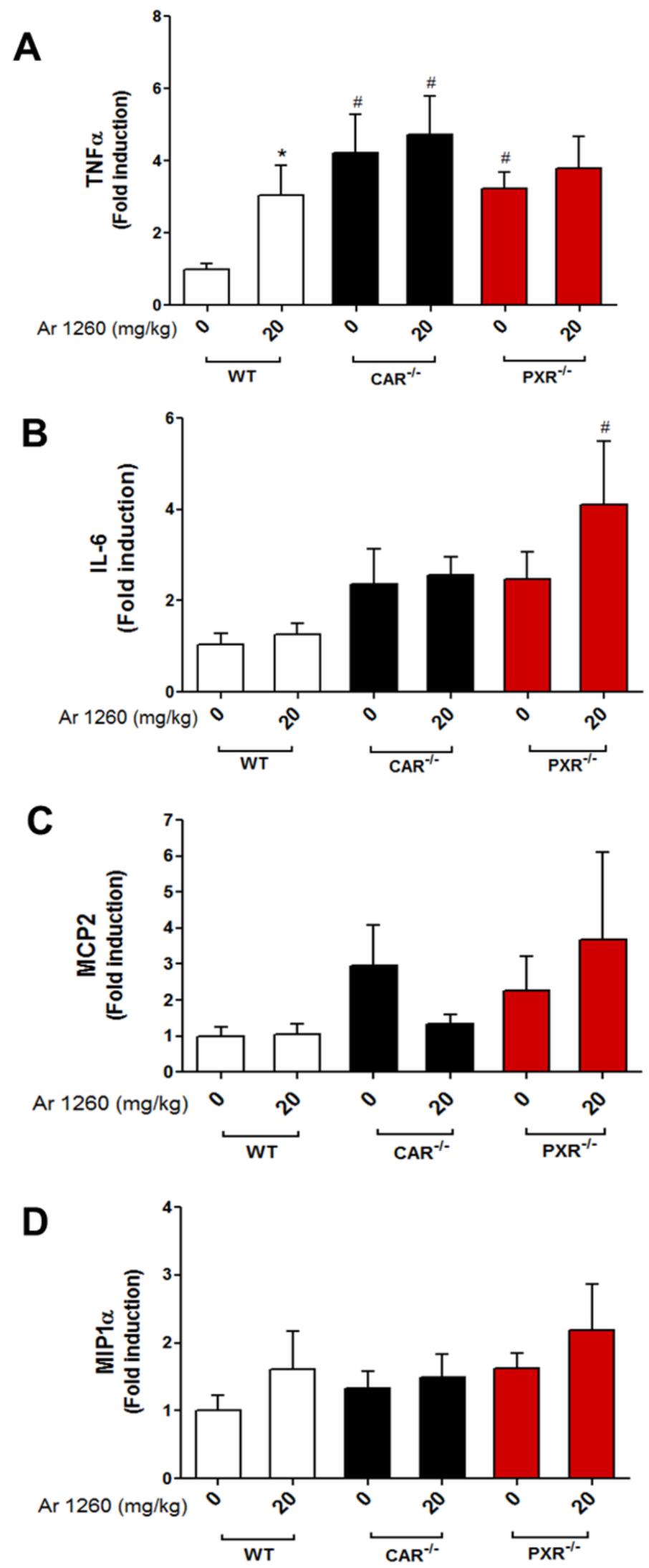
Figure 3.6. Effects of Aroclor 1260 exposure on the TLR-4 target genes in $\mathrm{CAR}^{-/-}$and $\mathrm{PXR}^{-/-}$mice.

Real-time PCR experiments showed the changes in hepatic mRNA expression caused by Aroclor 1260 exposure and knocking out CAR or PXR for (A) TNFa, (B) IL-6, (C) MCP2 and (D) MIP1 $\alpha$. Values are mean \pm SEM, $p<0.05$, * - $\Delta$ due to Aroclor 1260 exposure, \# - $\Delta$ due to knocking out CAR or PXR. 

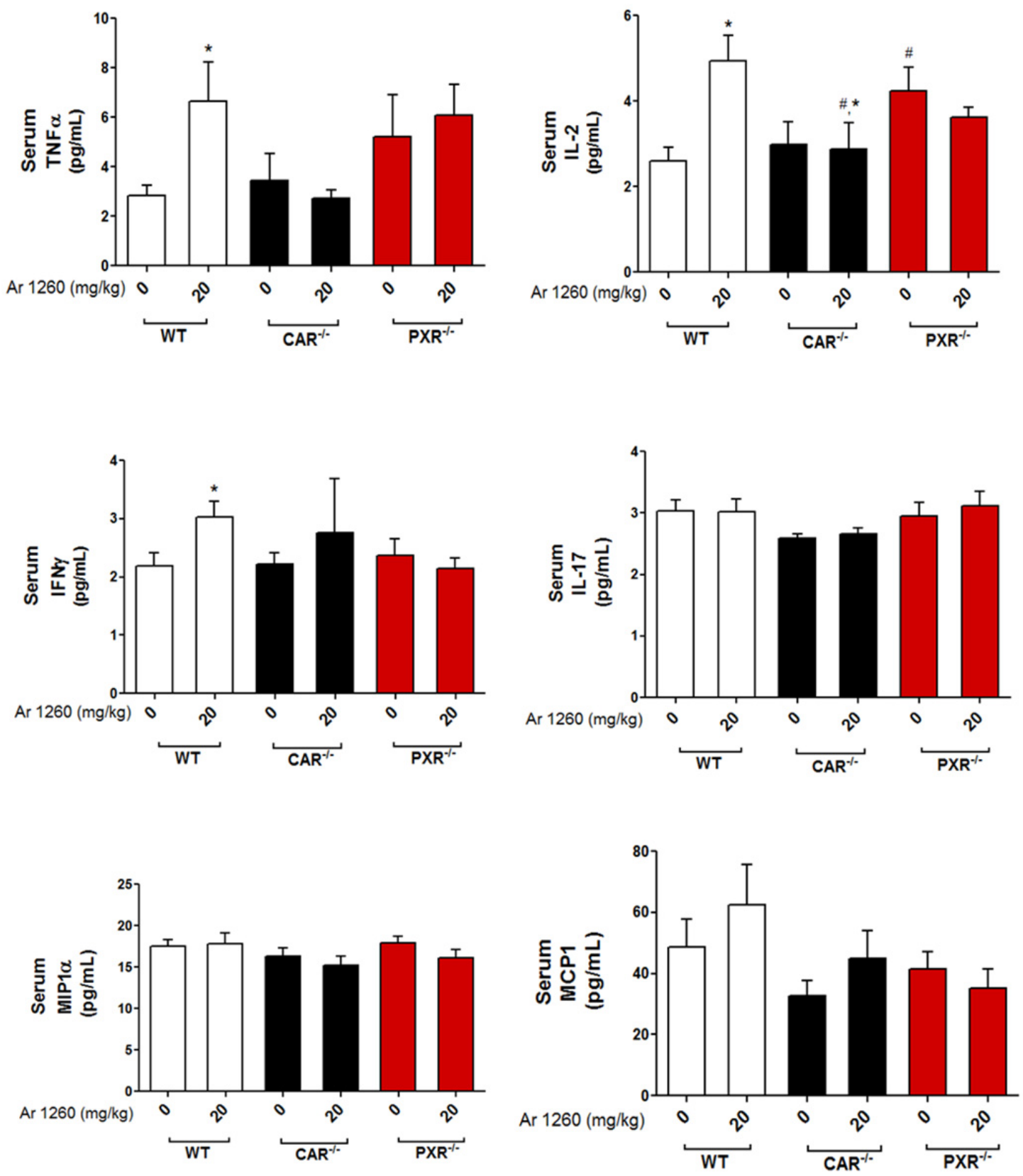
Figure 3.7. Effects of Aroclor 1260 exposure on serum cytokines in $\mathrm{CAR}^{-/-}$ and $P X R^{-/-}$mice.

Serum TNFa (pg/mL), IL-2 (pg/mL), IFNY (pg/mL), IL-17 (pg/mL), MIP1 $\alpha$ $(\mathrm{pg} / \mathrm{mL})$ and MCP1 $(\mathrm{pg} / \mathrm{mL})$ levels were measured using the Luminex IS 100 system $(n=10)$. Values are mean \pm SEM, $p<0.05,{ }^{*}-\Delta$ due to Aroclor 1260 exposure, \# - $\Delta$ due to knocking out CAR or PXR. 


\section{Aroclor 1260, CAR and PXR regulation in glucose metabolism and insulin resistance}

Fasting blood glucose levels were measured prior to performing the glucose tolerance test. There were no differences in fasting blood glucose levels between the six groups (Fig. 3.8 A). Glucose tolerance test was then performed and the area under the curve (AUC) was calculated to measure the degree of glucose uptake and clearance in the fed state (Fig. 3.8 B\&C). Aroclor 1260 exposure had no effect on GTT (AUC) in WT and $\mathrm{CAR}^{-/-}$groups. In contrast, Aroclor 1260 exposure caused an increase in the GTT (AUC) in $\mathrm{PXR}^{--}$mice. Insulin resistance was calculated by homeostasis model assessment using the formula: homeostasis model assessment of insulin resistance (HOMA$I R)=$ Fasting glucose $(\mathrm{mg} / \mathrm{dL}) \times$ Fasting insulin $(\mu \mathrm{U} / \mathrm{mL}) / 405$. HOMA-IR was calculated to determine if CAR and PXR played a role in high fat diet-induced insulin resistance, a common hallmark of non-alcoholic fatty liver disease (Fig. 3.8 D). Aroclor 1260 exposure significantly decreased HOMA-IR in WT, CAR ${ }^{-1-}$ and PXR ${ }^{-/-}$groups, although fasting blood glucose levels were not decreased. The reduction in HOMA-IR was due to lower serum insulin levels in all groups exposed to Aroclor 1260 (Fig. 3.8 E). These results indicated that Aroclor 1260 exposure resulted in impaired insulin production and thus, although GTT (AUC) was unchanged, HOMA-IR was decreased. Insulin sensitivity was assessed using the quantitative insulin sensitivity check index (QUICKI) as follows:

QUICKI=1 / (log (fasting insulin $\mu \mathrm{U} / \mathrm{mL})+\log$ (fasting glucose $\mathrm{mg} / \mathrm{dL})$ ). Interestingly, the Aroclor 1260-exposed, PXR knockout group showed a 
significantly higher index for insulin sensitivity, indicating a significant interaction between Aroclor 1260 exposure and PXR ablation (Fig. 3.8 F).

Furthermore, to assess gluconeogenesis, the hepatic expression of the CAR/PXR indirect targets, phosphoenol-pyruvate carboxykinase 1 (PEPCK-1) and glucose-6-phosphatase (G6Pase) were measured (Fig.3.9 A\&B). Hepatic PEPCK-1 m RNA levels were not affected with Aroclor 1260 exposure in the WT group. However, Aroclor 1260 exposure in the knockout groups induced PEPCK1 and this induction was highest in the $\mathrm{PXR}^{-/}$group, which may explain the increase in fed-state glucose levels as indicated by the GTT (AUC). Additionally, the PXR knockout mice exposed to Aroclor 1260 also showed significantly higher G6Pase expression in the liver. These data indicated that the gluconeogenic pathway was switched on in this group of mice, thus causing an increase in GTT (AUC).

Hepatic expression of the glycolytic enzyme glucokinase (Gck) was induced only in the CAR knockout mice exposed to Aroclor 1260 but not in the WT mice (Fig.3.9 C). This could potentially explain the increase in RER with Aroclor 1260 exposure in the CAR knockout groups. Hepatic expression of the glucose transporter GLUT-2 was increased with Aroclor 1260 exposure in the WT and CAR knockout groups but the induction by Aroclor 1260 was lost in the PXR knockout group (Fig. 3.9 D). On the other hand, the basal expression of the insulin-dependent glucose transporter, GLUT4 was higher in the CAR knockout groups but the mRNA levels were decreased with Aroclor 1260 exposure (Fig. $3.9 \mathrm{E})$. 

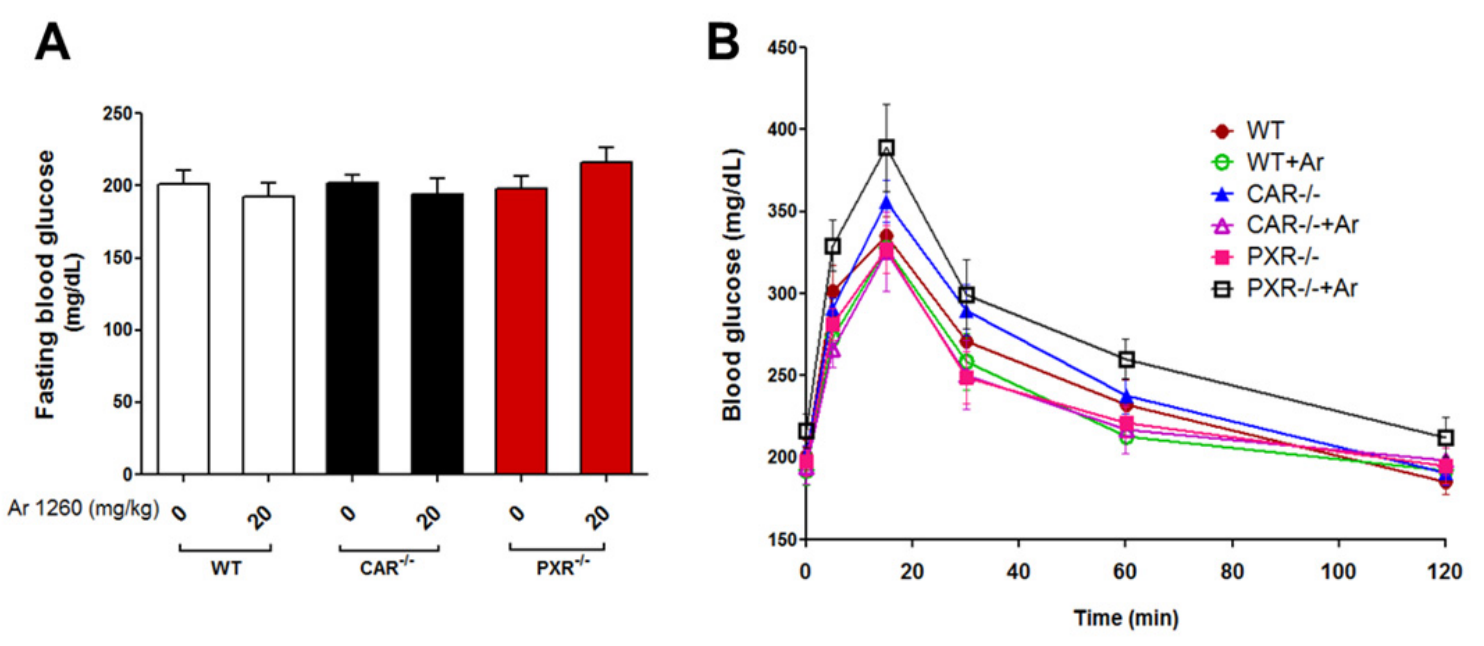

\section{C}
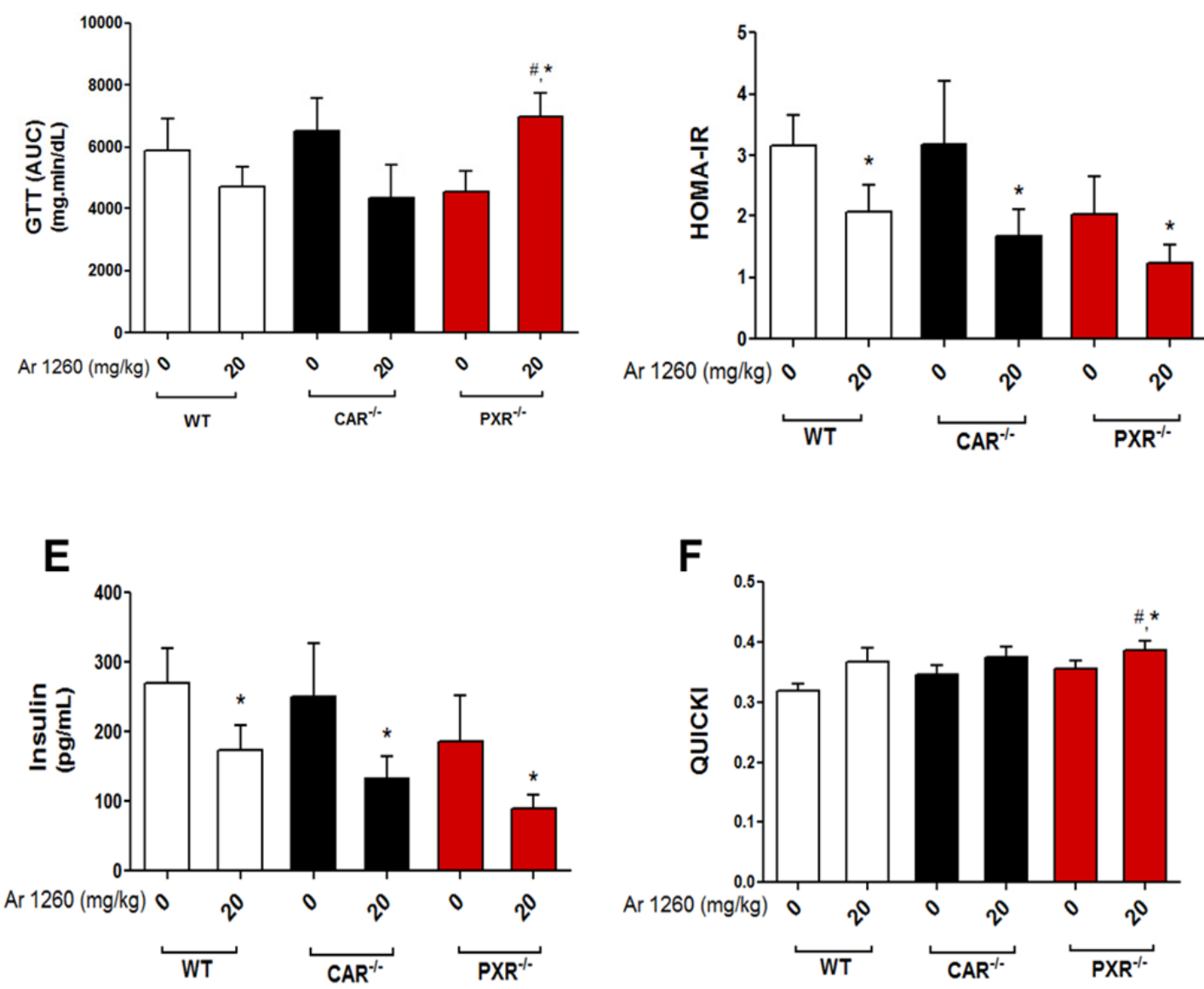
Figure 3.8. Effects of Aroclor 1260, CAR and PXR in glucose metabolism and insulin resistance.

(A) Fasting blood glucose levels ( $\mathrm{mg} / \mathrm{dL}$ ) were measured and (B) glucose tolerance test was performed. Blood glucose levels were measured for mice $(n=10)$ fed with high fat diet with or without Aroclor 1260 co-exposure. (C) AUC was calculated, and the PXR knockout mice exposed to Aroclor 1260 showed higher AUC levels vs. unexposed group and WT exposed mice. (D) Homeostasis model assessment of insulin resistance (HOMA-IR) was calculated from fasting blood glucose and insulin levels for all six groups of animals $(n=10)$. (E) Serum insulin levels were measured using the Luminex IS 100 system. (F) Quantitative insulin sensitivity check index (QUICKI) which is an index for insulin sensitivity was calculated. Values are mean \pm SEM, $p<0.05,{ }^{*}-\Delta$ due to Aroclor 1260 exposure, \# - $\Delta$ due to knocking out CAR or PXR. 
A

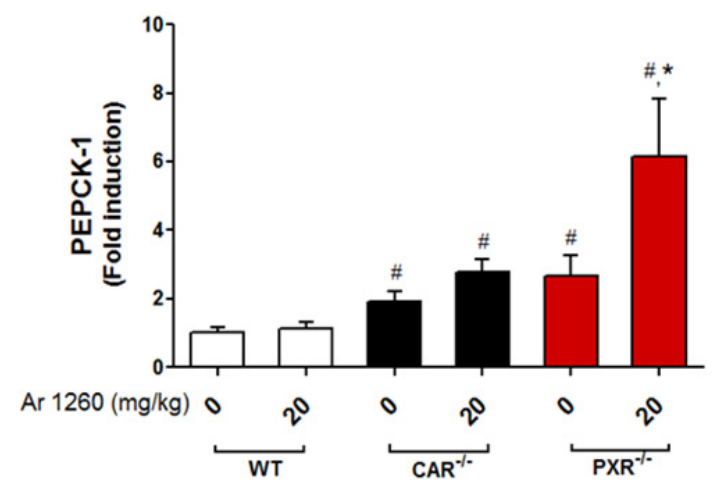

C

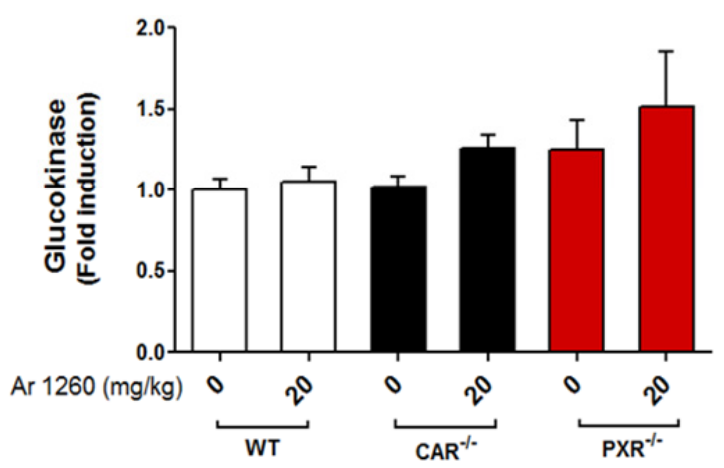

B

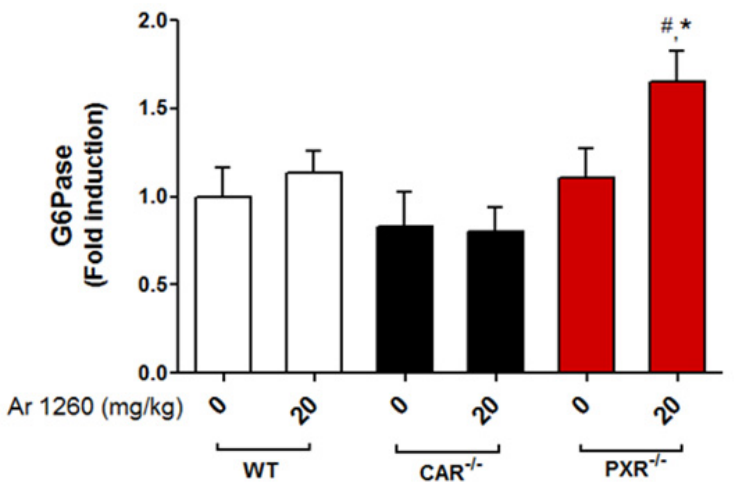

D

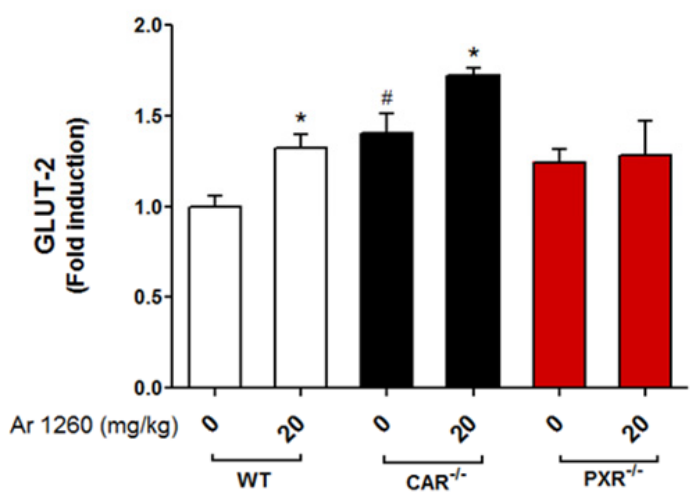

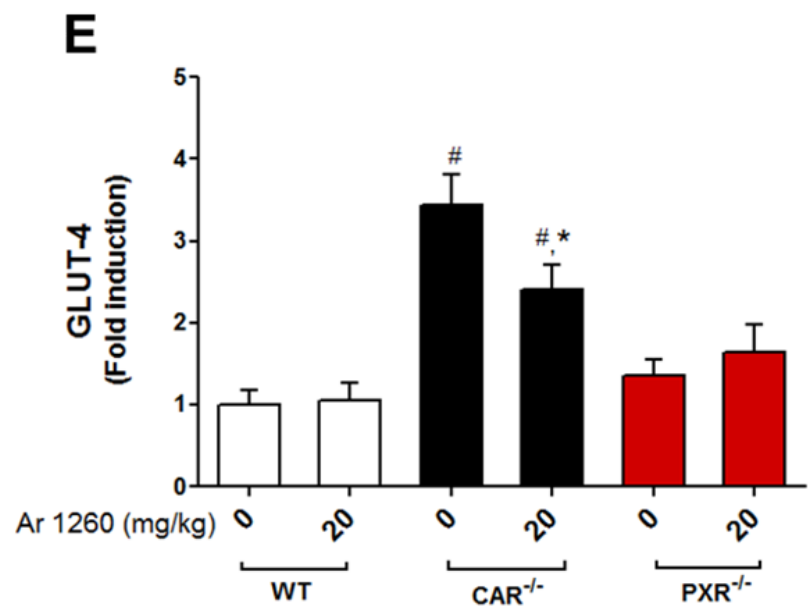


Figure 3.9. Effects of Aroclor 1260, CAR and PXR on genes involved in glucose metabolism.

Hepatic (A) PEPCK-1, (B) G6Pase, (C) glucokinase, (D) GLUT-2 and (E) GLUT-4 mRNA levels were quantified by RT-PCR. Values are mean \pm SEM, $p$ $<0.05,{ }^{*}-\Delta$ due to Aroclor 1260 exposure, $\#-\Delta$ due to knocking out CAR or PXR. 


\section{CAR and PXR ablation altered serum adipokine levels}

Serum adipokines were measured to evaluate the effects of knocking out CAR/PXR on the adipose tissue. Serum leptin levels were not changed with either Aroclor 1260 exposure or by knocking out CAR/PXR. In contrast, serum adiponectin levels were increased in the knockout mice, regardless of Aroclor 1260 exposure, leading to a decreased leptin/adiponectin ratio in the knockout mice (Fig.3. 10 A). This finding was consistent with the decreased adiposity observed in the CAR knockout group. However, the decreased leptin/adiponectin ratio was a paradoxical finding in the PXR knockout groups because the mice in these groups did not show a decrease in adiposity. Serum tissue plasminogen activator inhibitor-1 (tPAI-1) levels were not changed between the groups (Fig. 3.10 B). Serum lipids were also measured, including cholesterol and triglycerides (Fig. 3.10 C\&D). Serum cholesterol levels were higher in the unexposed, PXR knockout group whereas serum triglycerides levels were not altered between the groups. 

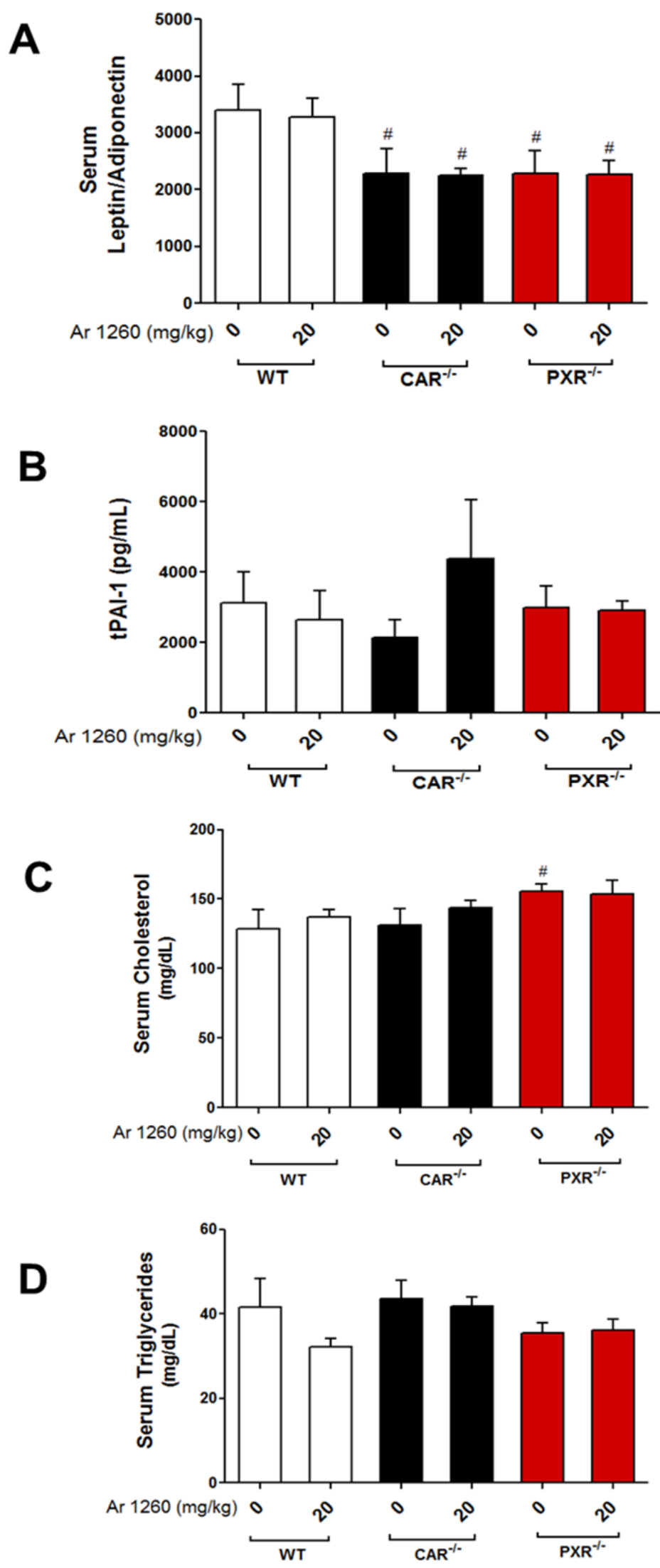
Figure 3.10. CAR/PXR knockout mice demonstrated altered serum adipokine levels.

(A) Serum leptin $(\mathrm{pg} / \mathrm{mL})$ and adiponectin $(\mathrm{pg} / \mathrm{mL})$ were measured using the Luminex IS 100 system and the leptin to adiponectin ratio was calculated. (B) Serum tPAI-1 levels were measured using the Luminex IS 100 system. Serum (C) cholesterol and (D) triglycerides were quantified with the Piccolo Xpress chemical analyzer. Values are mean \pm SEM, $p<0.05,{ }^{*}-\Delta$ due to Aroclor 1260 exposure, \# - $\Delta$ due to knocking out CAR or PXR. 


\section{Aroclor 1260, CAR and PXR modulated hepatic lipid metabolism and energy expenditure}

Liver-X-receptor alpha $(L X R \alpha)$ is a key transcription factor in lipid synthesis and cholesterol metabolism and its over-activation can promote or worsen steatosis. In the previous study, we demonstrated that Aroclor 1260 exposure modulated hepatic fat metabolism by either upregulation or downregulation of genes involved in fatty acid synthesis and breakdown. Moreover, CAR and PXR can bind to the direct repeat 4 (DR-4) elements which are similar to the response elements that LXRa binds to in the promoter regions of target genes. Therefore, looking at LXRa target genes in this study may determine if crosstalk between these three receptors is a significant interaction altering steatosis.

The hepatic expression of fatty acid synthase (FAS), a classic LXRa target gene was decreased with Aroclor 1260 exposure in WT mice (Fig. $3.11 \mathrm{~A}$ ). In contrast, FAS mRNA levels were up-regulated in the PXR knockout groups with or without Aroclor 1260 exposure. Similar to FAS, the expression levels of stearoyl coenzyme A desaturase1 (SCD1), another LXRa target gene, was decreased with Aroclor 1260 exposure in the WT group (Fig. 3.11 B). However, SCD1 expression was upregulated in the CAR knockout groups with or without Aroclor 1260 exposure.

The hepatic expression of CD36, a fatty acid binding protein and a common target gene of LXRa, PXR, AhR and PPARy, was also assessed (Fig. 3.11 C). Interestingly, Aroclor 1260 exposure resulted in an increase in CD36 
expression in the WT group compared to the unexposed mice. The $\mathrm{PXR}^{-/}$groups showed relatively higher basal CD36 mRNA levels than any other group and Aroclor 1260 exposure significantly induce CD36 in $\mathrm{PXR}^{-/-}$mice. The hepatic expression of the fatty acid binding protein 1 (FABP1), another protein required for fatty acid uptake and transport across the cell membrane was increased in the CAR knockout groups and the exposed, PXR knockout group (Fig. 3.11 D). Additionally, apart from the LXRa target genes that are involved in fat synthesis, hepatic expression of the lipolytic gene, papatin like phospholipase domain containing 2 (PNPLA2) was measured. Aroclor 1260 had no effect on PNPLA2 expression in WT mice (Fig. 3.11 E). However, Aroclor 1260 exposure induced PNPLA2 in CAR ${ }^{-/-}$mice. In contrast, Aroclor 1260 exposure did not induce PNPLA2 in $\mathrm{PXR}^{-/-}$mice but the basal levels of PNPLA2 expression were elevated.

The sterol regulatory element binding protein (SREBP-1c) is a transcription factor required for fatty acid biosynthesis and another transcriptional regulator for lipogenic genes including FAS and SCD1. SREBP-1c cleavage to its active form and subsequent translocation to the nucleus is controlled by many factors. One of these factors is CAR and PXR inhibition of SREBP-1c cleavage to its active form in the endoplasmic reticulum through the anti-lipogenic gene, Insig (73). Insig binds to the SREBP cleavage activating protein (SCAB) and prevents the cleavage of the SREBP precursor protein to its active form (112). We hypothesized that CAR and PXR would induce Insig and subsequently inhibit SREBP-1c cleavage to its active form (Fig. 3.12 A). Hepatic expression of 
SREBP-1c was analyzed and the PXR ${ }^{-/}$mice exposed to Aroclor 1260 exhibited higher SREBP-1c mRNA levels (Fig. 3.12 B). Hepatic expression of Insig-1 and 2 isoforms were therefore measured. Insig-1 mRNA levels were lower in the Aroclor 1260 exposed mice in the WT and PXR knockout groups (Fig. 3.12 C). Interestingly, CAR and PXR ablation also lowered Insig-1 hepatic expression. In contrast, Insig-2 expression levels did not differ among the groups (Fig. 3.12 D). To further investigate the underlying mechanisms that resulted in decreased lipogenic gene expression (FAS, SCD1) in the exposed WT group but not in the knockout groups, we measured the protein levels of SREBP1 in the mouse liver (Fig. 3.12 E\&F). Contrary to our hypothesis, CAR and PXR activation by Aroclor 1260 in the WT group did not result in lowered active SREBP1 isoform. Rather, Aroclor 1260 exposure caused an increase in SREPB1 cleavage from the inactive, precursor form that binds to the SREBP cleavage activating protein (SCAB)-Insig complex.

However, as mentioned before, SREBP1 transcriptional activity is also regulated by other factors such as the mammalian target of rapamycin complex 1 (mTORC1) (113). The total and phosphorylated mTOR1 protein levels in the liver were quantified and the phosphorylated to total mTOR1 ratio which was indicative of mTOR1 activation was calculated (Fig. 3.13 A). The knockout groups demonstrated an increase in mTOR1 activation irrespective of Aroclor 1260 exposure. The phosphorylated to total AMP-activated protein kinase $\alpha$ (AMPKa) protein levels were also quantified to assess AMPKa activation (Fig. 
3.13 B). Aroclor 1260 exposure activated AMPKa in the WT and PXR knockout mice. The CAR knockout mice showed an increase in basal AMPKa active form. 
A

B

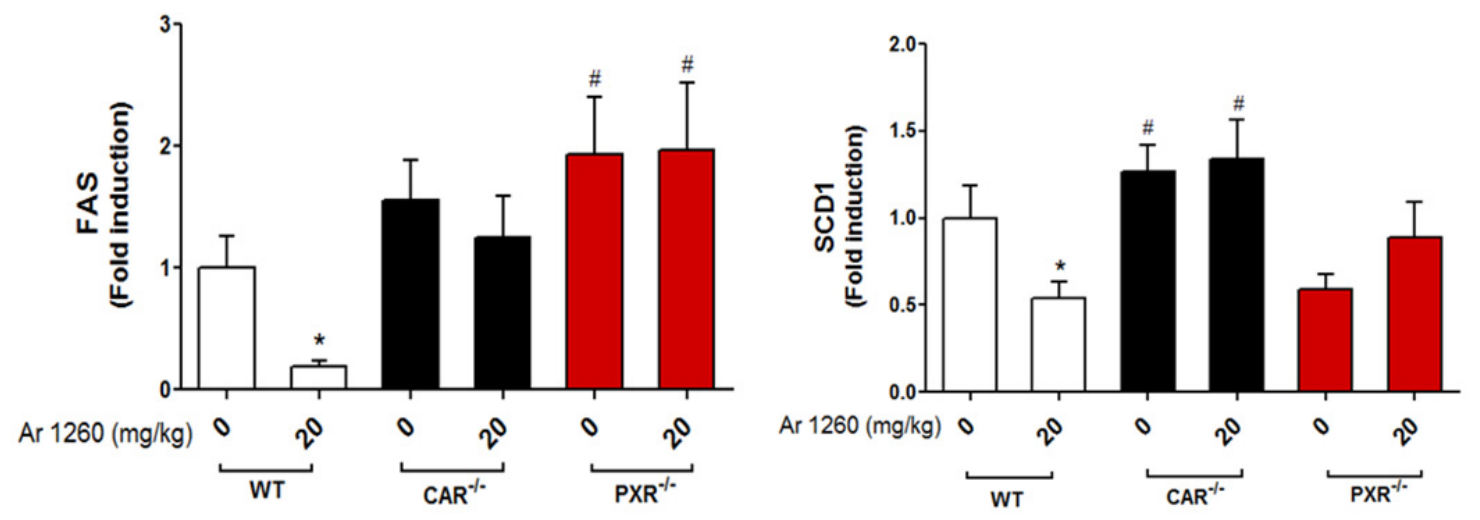

C

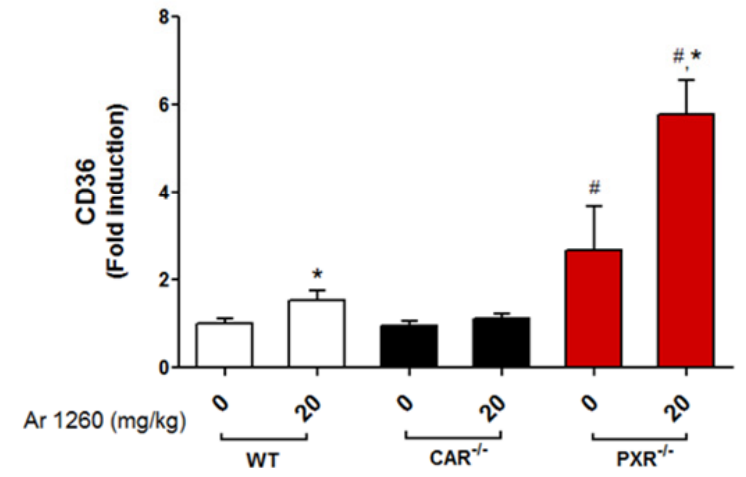

D

E

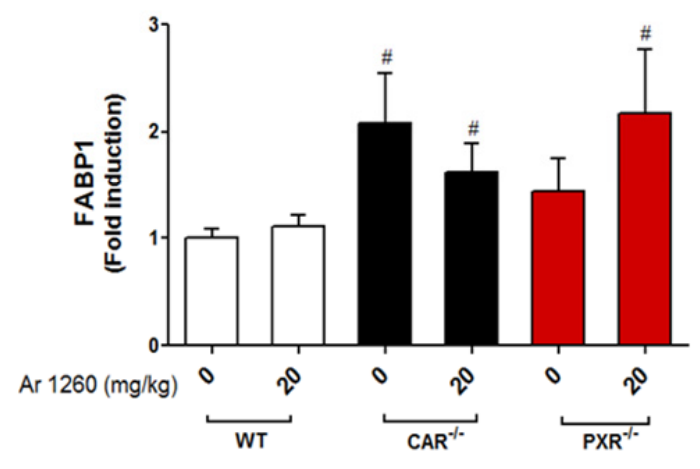

E

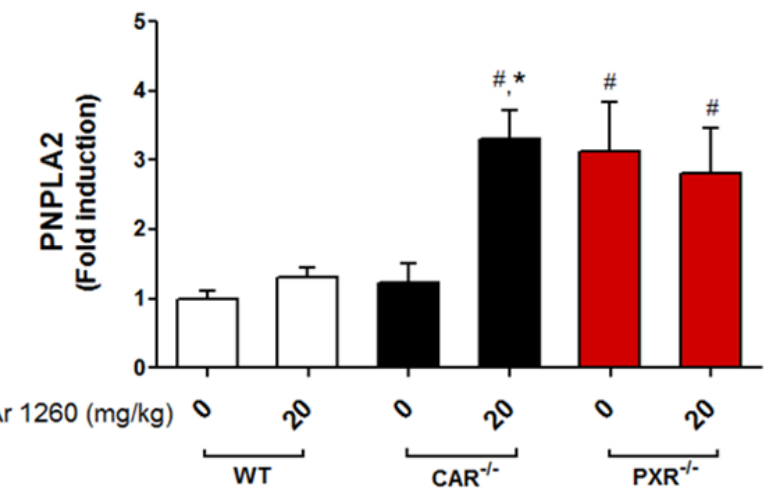


Figure 3.11. Effects of Aroclor 1260, CAR and PXR on hepatic expression of lipogenic and lipolytic genes.

Real-time PCR experiments showed the changes in hepatic mRNA expressions caused by Aroclor 1260 exposure alone and/ or by ablating CAR or PXR for (A) FAS, (B) SCD1, (C) CD36, (D) FABP1 and (E) PNPLA2. Values are mean \pm SEM, $p<0.05,{ }^{*}-\Delta$ due to Aroclor 1260 exposure, \# - $\Delta$ due to knocking out CAR or PXR. 
A

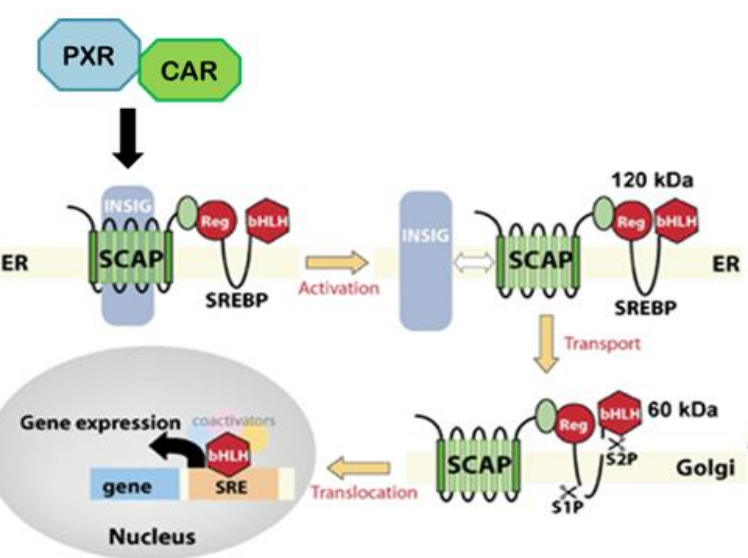

C

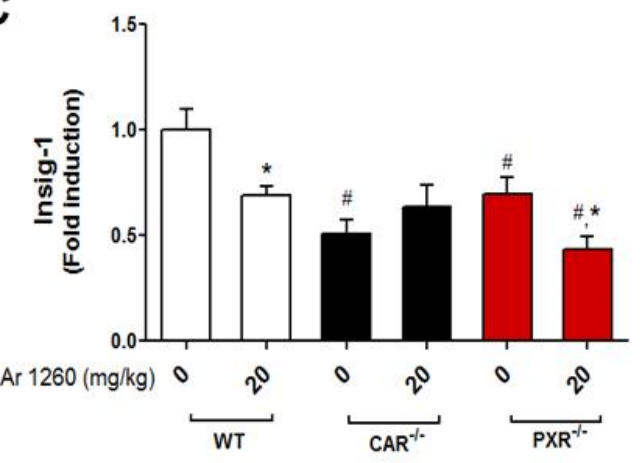

B

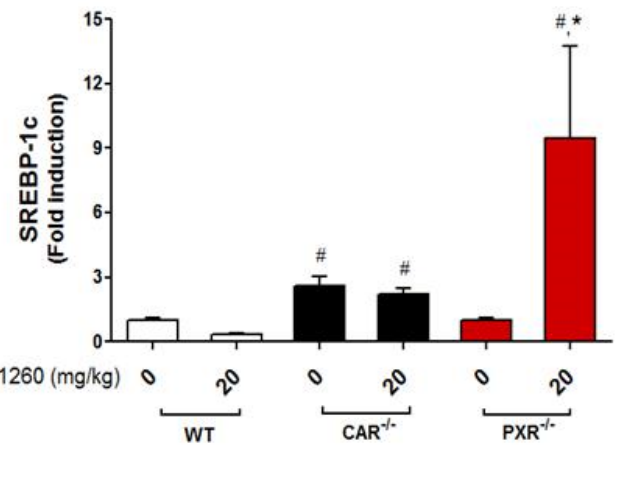

E
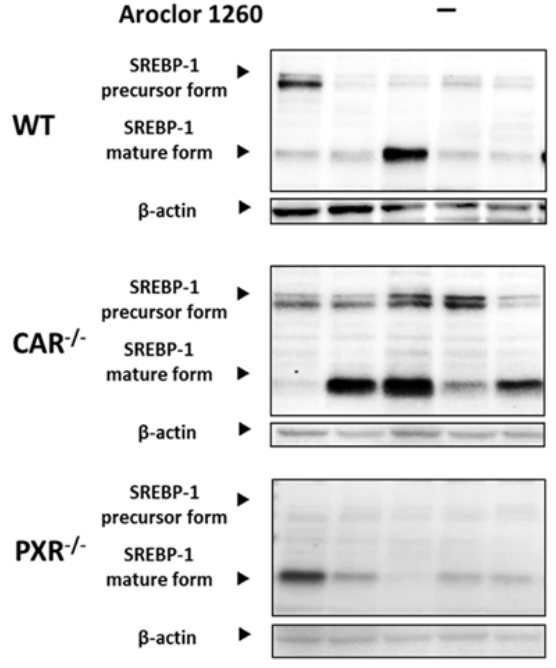

$+$
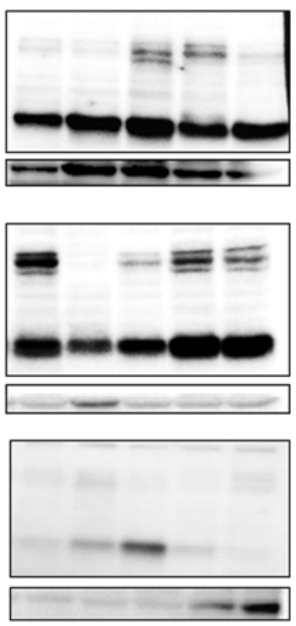

F

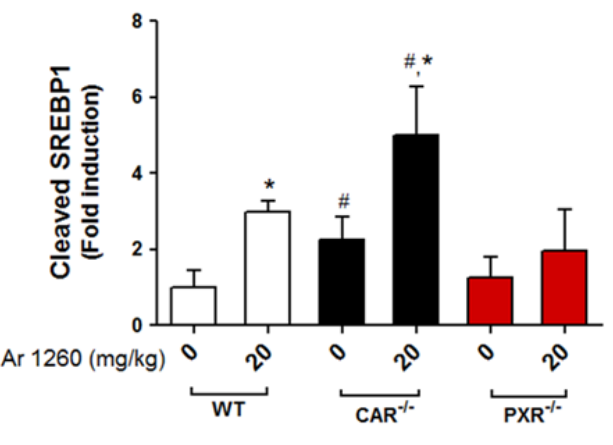


Figure 3.12. Effects of Aroclor 1260, CAR and PXR on hepatic SREBP-1c expression and protein levels.

(A) Schematic diagram depicting CAR and PXR regulation on SREBP through Insig. The diagram was adapted from Hellard et al., Molecular Psychiatry, 14, 2009. Real-time PCR experiments showed the changes in hepatic mRNA expressions caused by Aroclor 1260 exposure alone and/or by ablating CAR or PXR for (B) SREBP-1C, (C) Insig-1 and (D) Insig-2. (E \& F) Immunoblots for precursor and cleaved SREBP1 were performed, $n=5$. Active SREBP1 protein levels were quantified. Values are mean \pm SEM, $p<0.05,{ }^{*}-\Delta$ due to Aroclor 1260 exposure, \# - $\Delta$ due to knocking out CAR or PXR. 

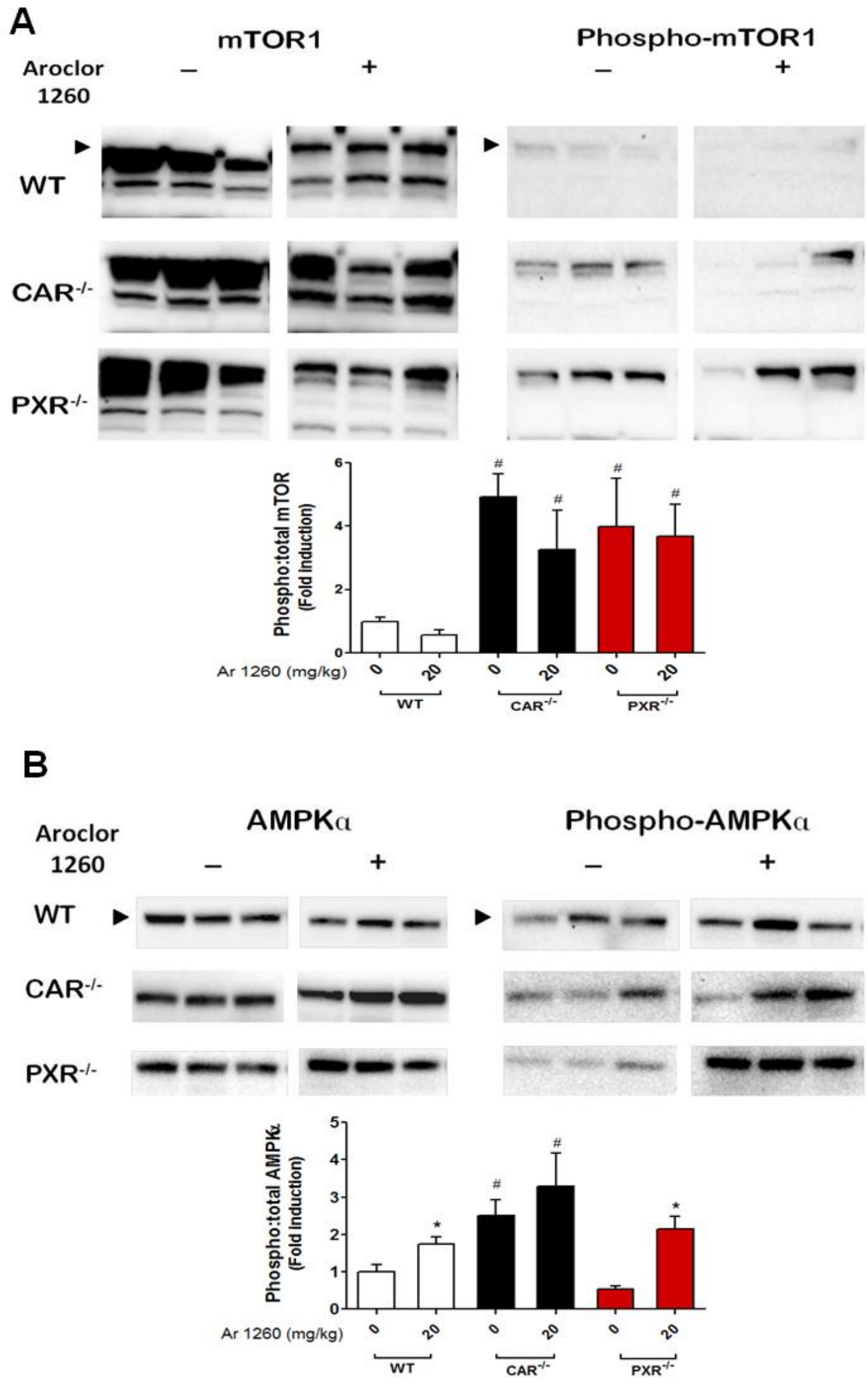
Figure 3.13. Effects of Aroclor 1260, CAR and PXR on mTOR1 and AMPKa protein levels.

(A) Immunoblots for mTOR1 and phosphorylated mTOR1 were performed. The phosphorylated to total mTOR1 ratio was quantified. (B) Immunoblots for AMPKa and phosphorylated AMPKa were performed. The phosphorylated to total AMPKa ratio was quantified. Values are mean $\pm \mathrm{SEM}, p<0.05,{ }^{*}-\Delta$ due to Aroclor 1260 exposure, \# - $\Delta$ due to knocking out CAR or PXR. 


\section{Effects of CAR/PXR on peroxisome proliferator-activated receptor alpha target genes}

Peroxisome proliferator-activated receptor alpha (PPAR $\alpha$ ) drives the transcription of genes involved in breaking down fatty acids such as carnitine palmitoyl transferase (CPTs) and Cyp4a10. Hepatic expression of PPARa as well as its target genes, CPT1A and Cyp4a10 was measured. The PPAR $a$ mNA levels were higher in the Aroclor 1260-exposed mice compared to the unexposed mice in the WT and $\mathrm{PXR}^{-/}$groups (Fig. 3.14 A). Although unexposed $\mathrm{CAR}^{-/-}$mice had increased PPARa mRNA levels vs. unexposed WT mice, Aroclor 1260 had no effect on PPARa expression in $\mathrm{CAR}^{-/-}$mice. In contrast, CPT1A was induced with Aroclor 1260 in all three groups irrespective of CAR/PXR ablation (Fig. 3.14 B). Additionally, the Aroclor 1260-exposed $\mathrm{PXR}^{-/-}$mice showed the highest induction, and there was a significant interaction between PXR ablation and Aroclor 1260 exposure which was consistent with PPARa induction. Furthermore, Aroclor 1260 induced Cyp4a10 only in $\mathrm{CAR}^{-/-}$mice (Fig. 3.14 C). Also, PXR ablation induced Cyp4a10 in the exposed mice. 

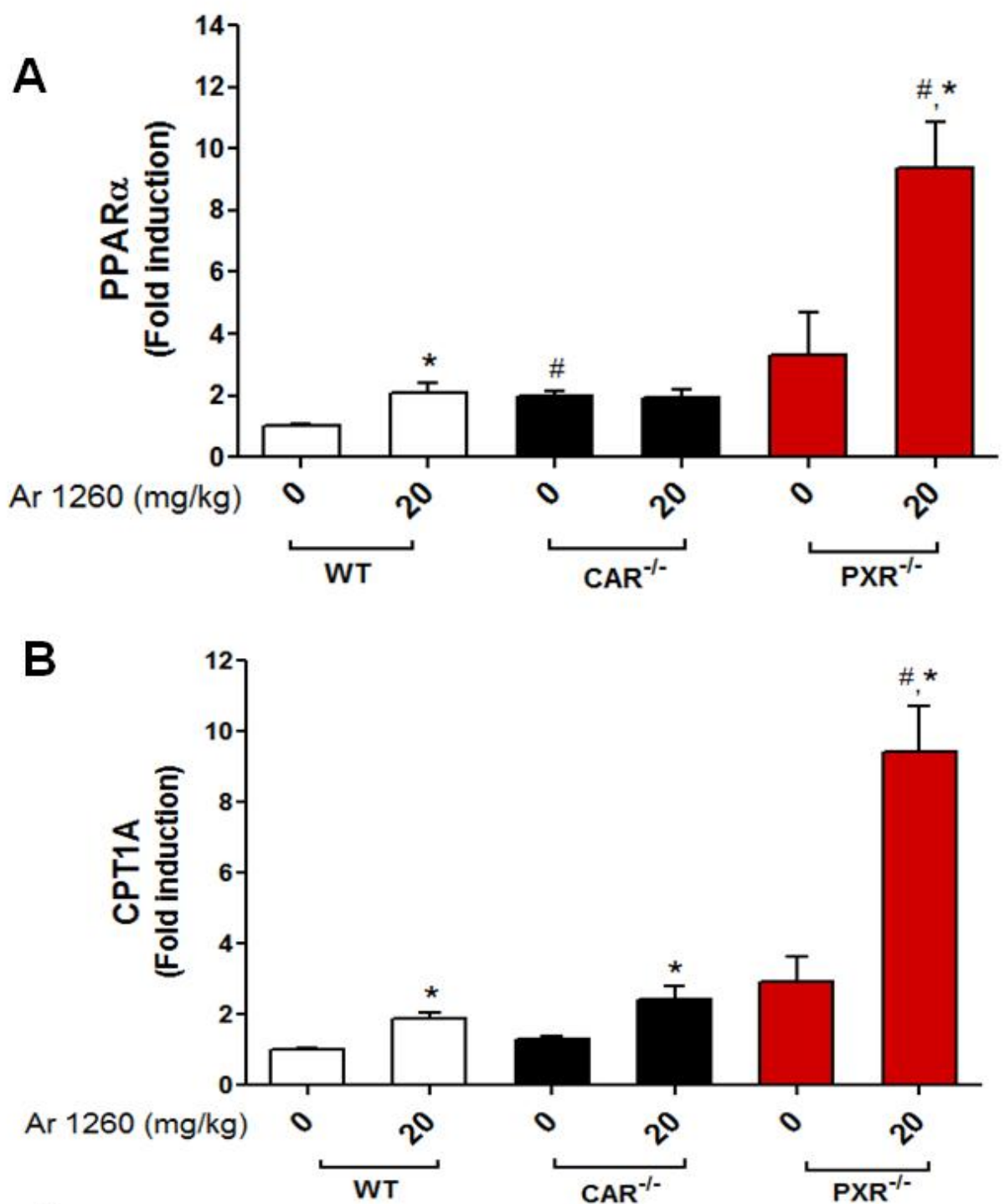

C

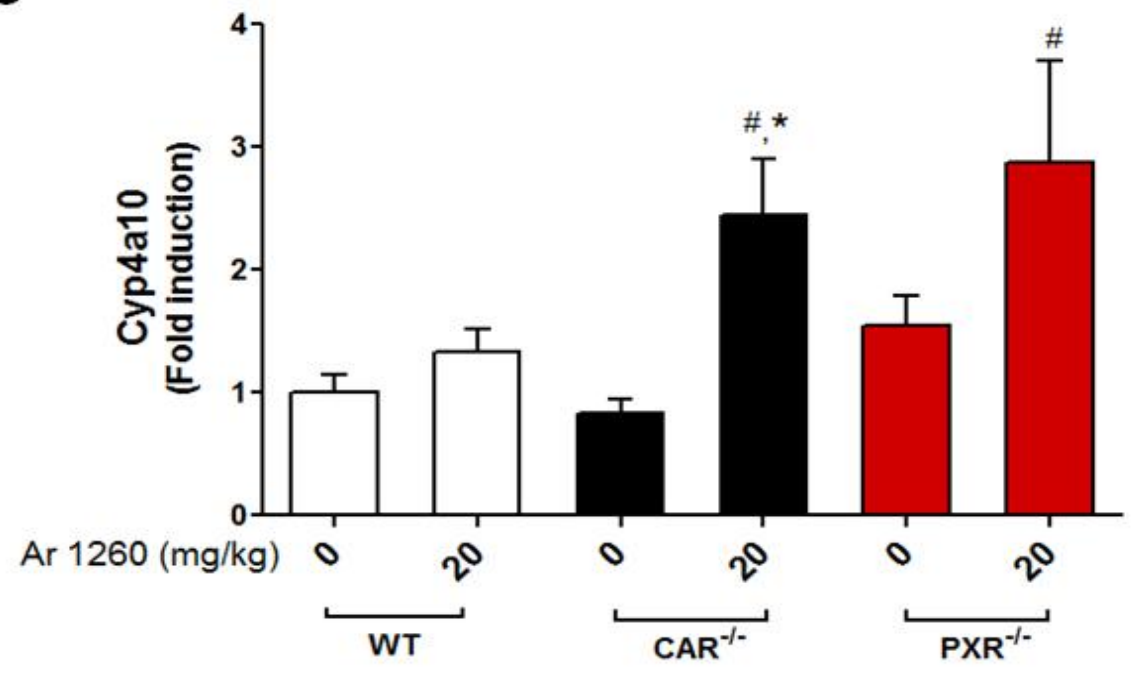


Figure 3.14. Effects of Aroclor 1260 exposure and CAR/PXR ablation on hepatic PPARa expression and its target genes.

Real-time PCR experiments showed the changes in hepatic mRNA expressions caused by Aroclor 1260 exposure alone or by ablating CAR or PXR as well for (A) PPARa, (B) CPT1A and (C) Cyp4a10. Values are mean \pm SEM, $p$ $<0.05,{ }^{*}-\Delta$ due to Aroclor 1260 exposure, $\#-\Delta$ due to knocking out CAR or PXR. 


\section{CAR and PXR target gene induction}

CAR hepatic mRNA levels were measured and as anticipated, there was no CAR mRNA in the $\mathrm{CAR}^{-/}$mice. Aroclor 1260 exposure increased CAR mRNA expression ( 2-fold) in WT mice (Fig. 3.15 A). PXR ablation also increased CAR mRNA expression by $\sim 10$-fold as compared to WT groups. There was also a significant interaction between PXR ablation and Aroclor 1260 exposure in CAR induction. The mRNA levels of Cyp2b10, a CAR target gene, were significantly up-regulated in Aroclor 1260-exposed mice in the WT and PXR ${ }^{-/}$groups (Fig. 3.15 B). Moreover, the basal mRNA levels of Cyp2b10 were higher in the knockout groups, indicating a compensatory mechanism was driving CAR target gene expression in the absence of CAR. Notably, Aroclor 1260 did not induce Cyp2b10 in the $\mathrm{CAR}^{-/-}$group.

Hepatic expression of PXR was also evaluated, and as expected, there was no PXR mRNA in the PXR ${ }^{-/}$mice (Fig. 3.15 C). Aroclor 1260 did not induce PXR mRNA expression in the WT mice. In contrast Aroclor 1260 exposure did increase PXR expression ( 17-fold) in the $\mathrm{CAR}^{-/-}$mice. Hepatic Cyp3a11 (PXR target gene) mRNA levels were up-regulated with Aroclor 1260 exposure in the $\mathrm{CAR}^{-/-}$group but not in the WT group (Fig. 3.15 D). Akin to Cyp2b10 expression, the Сyp3a11 basal expression levels were higher in the knockout groups. However, Aroclor 1260 did not induce Cyp3a1 in the $\mathrm{PXR}^{-/-}$group. Besides Cyp3a11, the hepatic expression of Ugt1a1, a predominant PXR target gene which also happens to be a CAR target gene, was measured (Fig. 3.15 E). Aroclor 1260 induced Ugt1a1 in the WT and $\mathrm{CAR}^{-/}$groups. Interestingly, Ugt1a1 
basal expression levels were higher only in the in the $\mathrm{PXR}^{-/-}$group but not in the $\mathrm{CAR}^{-/}$group.

Apart from CAR and PXR targets, the hepatic expression of Cyp1a2, an aryl hydrocarbon receptor (AhR) target gene was also measured (Fig. 3.15 F). There were no significant differences in the Cyp1a2 mRNA levels between the groups indicating that the AhR was not activated by Aroclor 1260 at the dose used and there was no effect of CAR or PXR ablation on AhR target gene expression. 

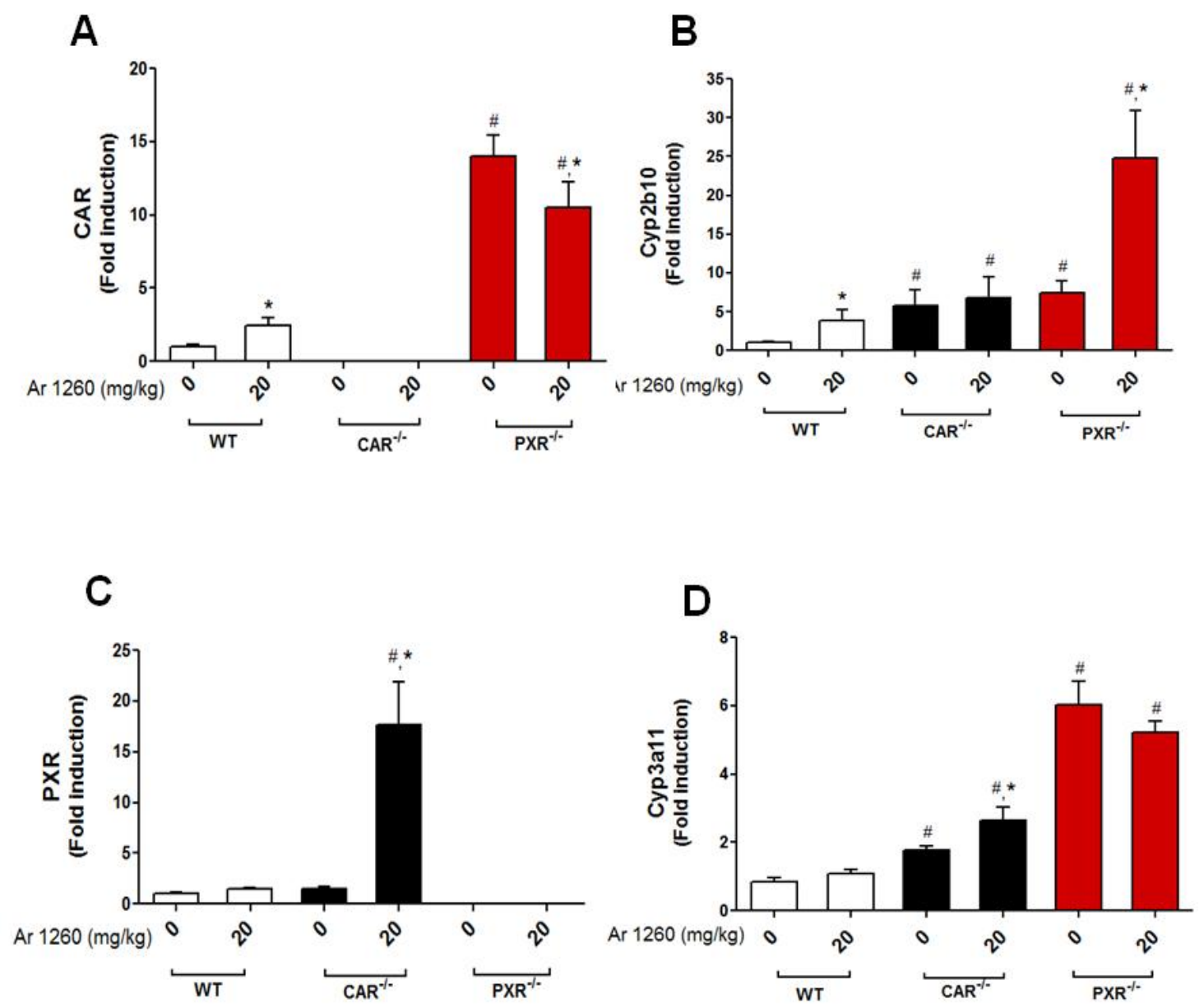

E

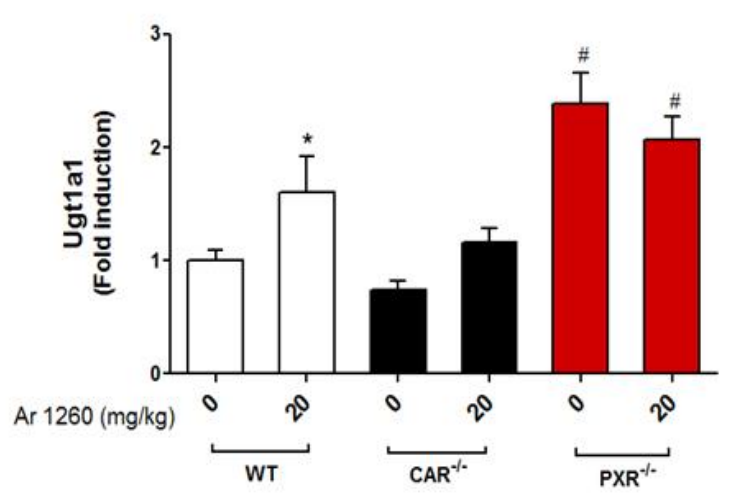

F

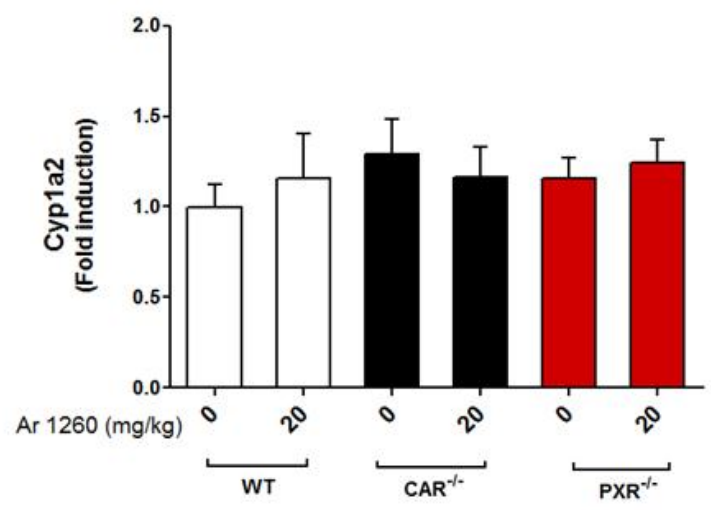


Figure 3.15. Aroclor 1260 exposure altered hepatic expression of CAR and PXR target genes.

Real-time PCR experiments showed the changes in hepatic mRNA expressions for (A), CAR, (B) Cyp2b10 (CAR target gene), (C) PXR, (D) Cyp3a11 (PXR target gene), (E) Ugt1a1 (PXR/CAR target gene)and (F) Cyp1a2 (AhR target gene). Values are mean \pm SEM, $p<0.05,{ }^{*}-\Delta$ due to Aroclor 1260 exposure, \# - $\Delta$ due to knocking out CAR or PXR. 


\section{DISCUSSION}

Aroclor 1260 was one of the first PCB mixtures manufactured and was later replaced by PCB mixtures with lower chlorine content. Although constituting only $11 \%$ of PCB mixtures manufactured, Aroclor 1260 was selected for this study based on its similarity to human PCB bioaccumulation patterns. The PCB congener composition in Aroclor 1260 consists of high molecular weight PCBs such as 5-, 6-, and 7-chlorinated congeners that are either not or very poorly metabolized so these congeners bio-accumulate in human and other biota. Most PCB studies in the past literature focused on occupational acute exposures that were associated with cancer-related endpoints and AhR activation. More recently, epidemiologic studies have revealed that PCB-exposed human cohorts showed signs of cardiovascular disorders, obesity, diabetes/insulin resistance and elevated liver enzymes $(46,52,114)$.

Clearly, chronic exposures to PCBs appear to disrupt lipid and glucose homeostasis and consequently lead to increased symptoms of diabetes and the metabolic syndrome in the exposed subjects $(34,52)$. Mechanistically, PCB action is thought to be mediated by the AhR and CAR. However, PCBs have also been shown to interact with endocrine and thyroid receptors as well as additional hepatic nuclear receptors including the PXR (97-99). Albeit being xenobiotic receptors, CAR and PXR are also involved in energy metabolism by acting directly or indirectly on enzymes involved in lipid and glucose metabolism (6062). We previously demonstrated that Aroclor 1260 induced liver injury in conjunction with HFD feeding. We also demonstrated that Aroclor 1260 activated 
CAR and PXR in C57BI/6 mice at $20 \mathrm{mg} / \mathrm{kg}$ exposure. The current study aimed at examining the role of CAR and PXR in Aroclor 1260-induced liver injury by using diet-induced obese CAR and PXR knockout mice.

The findings from the study demonstrated that PXR was important in maintaining lower fat mass and liver weight because ablating this receptor resulted in increased adiposity/liver weight (Fig. 3.1). CAR ablation did not appear to interfere with adiposity. In fact, Aroclor 1260 exposure decreased bodyweight gain in CAR knockout mice, which may be due to increased physical activity and less food consumption displayed by this group. Interestingly, PXR was also over-expressed in this group. Presence of CAR and PXR also appeared crucial in maintaining energy homeostasis because ablating these receptors decreased the RER relative to WT mice, indicating only a lipid-metabolism state (Fig. 3.3). However, activation of either receptor by Aroclor 1260 restored the RER to a level similar to WT groups.

When inflammation was examined, CAR and PXR ablation did not improve steatohepatitis induced by Aroclor 1260. Both CAR and PXR knockout mice showed signs of hepatic inflammation on high fat diet feeding alone, as evident by the TNFa and IL-6 basal expression levels (Fig. 3.6). These results suggested that both CAR and PXR play an inhibitory role in inflammation and activating these receptors by Aroclor 1260 is a protective mechanism in attenuating Aroclor 1260 -induced toxicity. The role of PXR in inflammation is well documented relative to CAR (115). It is well known that exposure to xenobiotic chemicals such as rifampicin compromises the immune function and recent 
studies have investigated the molecular mechanisms involved between PXR activation and inflammation. Zhou et al demonstrated that the interaction between PXR and nuclear factor kappa B (NF-KB) appeared to be reciprocal with both transcription factors mutually inhibiting each other (116). Enhanced expression of NF-KB target genes including TNFa, IL-2 and IL-6 was observed in the small bowel and liver of PXR knockout mice which was consistent with observations in the current study. Hu et al also showed increased hepatic expression of inflammatory cytokines in PXR knockout mice and demonstrated that liganded PXR was SUMOylated in hepatocytes in response to TNFa (117). Intriguingly; the SUMOylated PXR protein suppressed NF-KB target gene expression but had no effect on PXR target gene expression such as CYP3A4. The relationship between CAR and the inflammatory process is poorly understood, although some observations have implied its basal repression activity on NF-kB signaling (115). In the present study, it should be noted that even though Aroclor 1260 activated CAR/PXR in WT mice, their activation was not sufficient to protect the mice from hepatic and systemic inflammation, implying that Aroclor 1260 may affect liver injury by mechanisms other than the classically-defined receptor mechanisms.

Cell culture studies indicated that activators of CAR and PXR repress hepatic gluconeogenic enzymes and therefore, CAR/PXR modulation of glucose metabolism possibly resulted in lowered blood glucose levels $(71,75)$. CAR and PXR inhibit gluconeogenic gene transcription, including PEPCK-1 and G6Pase, by directly binding to the transcription factor, forkhead boxO1 (FOXO1) and 
preventing its transcriptional activity (74). CAR can also interfere with gluconeogenic gene expression by competing with the hepatocyte nuclear factor 4 alpha (HNF4 $\alpha$ ) for binding at the PEPCK-1 promoter region. Also, CAR and PXR target the common co-activator utilized by numerous transcription factors, peroxisome proliferator-activated receptor gamma coactivator 1 alpha (PGC1 1 ), resulting in the 'squelching' of PGC1a $(118,119)$.

In the current study, hepatic expression of PEPCK-1 was enhanced in both CAR and PXR knockout mice (Fig. 3.9), implicating the loss of CAR- and PXR-inhibitory effect on PEPCK-1 transcription. PEPCK-1 was also induced with Aroclor 1260 exposure in the knockout mice, noticeably in $\mathrm{PXR}^{-/-}$mice, suggesting that PCB exposure increased gluconeogenic gene expression in absence of either CAR or PXR. Although fasting blood glucose levels were unchanged, all Aroclor 1260-exposed groups showed decreased serum insulin and HOMA-IR levels (Fig. 3.8). This observation is consistent with findings from our previous studies, associating compromised insulin secretion with PCB exposure, independent of CAR and/or PXR presence and activation. Thus the PXR knockout mice exposed to Aroclor 1260 showed increased AUC in a glucose tolerance test, potentially due to increased gluconeogenesis and decreased insulin synthesis required for glucose uptake.

Another compelling observation in this study was the decreased leptin:adiponectin ratio in CAR and PXR knockout mice (Fig. 3.10). Circulating leptin:adiponectin ratio is considered a potential surrogate biomarker for obesityrelated conditions with higher leptin levels being observed consistently with 
metabolic syndrome and diabetes/insulin resistance (120). In this study, serum adiponectin levels were higher in the knockout mice whereas leptin levels were not altered between the groups. Nonetheless, PXR knockout mice did not show any improvement in diet-induced obesity, despite the favorable leptin:adiponectin ratio while the CAR knockout mice displayed reduced adipocyte size.

Aroclor 1260 exposure resulted in decreased FAS expression in WT mice (Fig. 3.11). However, the PXR knockout mice showed increased FAS basal levels indicating that activated PXR repress FAS transcription. Likewise, SCD1 expression was decreased with Aroclor 1260 exposure; however its basal levels were increased only in CAR knockout mice suggesting that CAR could be a transcriptional repressor of SCD1. This supports other studies documenting decreased hepatic expression of lipogenic genes such as FAS and SCD1 during CAR activation $(71,72)$. Moreover, CAR suppression of LXRa transcriptional activity has also been reported, which could then cause downregulation of lipogenic gene expression (121). Additionally, Aroclor 1260 did not induce FAS or SCD1 in CAR knockout mice, therefore, this PCB mixture may not be a direct LXR $\alpha$ activator. In contrast, SREBP-1c was significantly induced only in PXR knockout mice exposed to Aroclor 1260, suggesting an interaction between PCB exposure and PXR ablation. However, simply measuring SREBP-1c expression alone may not be sufficient to determine the CAR/PXR effects on lipogenesis since both CAR and PXR also affect SREBP-1c activity through the anti-lipogenic gene, Insig (73). Therefore, it was more appropriate to measure active SREBP$1 \mathrm{c}$ levels in the liver. 
Contrary to our hypothesis, there was no reduction in active SREBP1 protein levels with Aroclor 1260 exposure (Fig. 3.12). Rather, the Aroclor 1260 exposed groups in the WT and CAR knockout mice had higher cleaved SREBP1 protein levels than the unexposed group. Additionally, since the exposed groups did not show an increase in hepatic Insig-2 expression either, it appeared that CAR/PXR activation and ablation did not modulate hepatic gene expression through the Insig2-SCAB-SREBP1 pathway. Notably, hepatic Insig-1 expression was decreased in the Aroclor 1260 exposed groups. Insig-1 regulates the SCABSREBP2 complex and its expression is regulated in part by nuclear SREBP levels and sterol deprivation (122). Furthermore, although it has been reported that CAR and PXR regulate lipogenesis by inducing Insig-1, the SREBP1 retention in the endoplasmic reticulum has also been reported to be via Insig-2 binding (123). However, Insig-2 expression did not differ among the groups.

CAR and PXR ablation appeared to activate the mTOR1 complex pathway and Aroclor 1260 exposure appeared to activate AMPKa (Fig. 3.13). These are intriguing observations because mTOR and AMPK are considered as 'energy sensors' that regulate carbohydrate and lipid metabolism, thereby maintaining energy homeostasis at the cell and whole body level $(124,125)$. mTOR activation occurs during high nutrient availability or 'fed' state and usually favors anabolic, ATP-consuming processes that facilitate storage of nutrients such as lipogenesis and protein synthesis. Regulation of lipogenic SREBP1 by mTOR1 occurs at multiple levels (126). It has been demonstrated that high insulin levels can activate the mTOR1 complex through the Akt phosphorylation pathway (127). 
Phosphorylated mTOR can phosphorylate the phosphatidic acid phosphatase Lipin-1, a transcriptional coactivator (113). Nuclear localized, dephosphorylated Lipin-1 inhibits SREBP1 nuclear localization and subsequently SREBP1 transcriptional activity. Hence mTOR1 activation eventually leads to increased SREBP1 nuclear localization and activity. In this study, mTOR1 activation in the knockout groups possibly resulted in observable inductions of lipogenic gene expression. Interestingly, the WT mice exposed to Aroclor 1260 showed lower FAS and SCD1 expression which corroborated with lower phosphorylated mTOR1 levels. Moreover, the Aroclor 1260-exposed mice had lower insulin levels and this may have led to decreased phosphorylated mTOR1 levels compared to the unexposed mice in the WT group. However, this effect was lost with CAR and PXR ablation and the mechanisms related to this observation need to be investigated further.

In contrast to mTOR1, AMPKa activation occurs during low nutrient availability or 'fasted' state and favors catabolic processes such as glycolysis (128). Besides, downstream AMPK signaling also inhibits anabolic processes that consume ATP. The mechanism involving AMPKa activation by Aroclor 1260 exposure in the current study is unclear. However, AMPKa activation could be the reason for the increase in RER in the Aroclor 1260 exposed, PXR knockout mice. The same explanation could not be applied to the CAR knockout group because CAR ablation appeared to activate AMPKa irrespective of Aroclor 1260 exposure. Also, it has been reported that metformin, an AMPK activator, inhibits CAR nuclear translocation and activation, indicating a complex relationship 
between CAR and AMPK (129). Therefore more studies are necessitated with regard to PCB exposure and the mTOR and AMPK activation pathway.

Aroclor 1260 induced CD36, a shared target gene of PXR, AhR and LXRa in WT mice (Fig. 3.11). It is known that PXR activators such as rifampicin can promote steatosis independent of the SREBP1 pathway by up-regulating hepatic CD36 (130). Interestingly, ablating PXR increased CD36 basal expression, implying that there may be some compensatory mechanisms affecting CD36 expression in the absence of PXR. Furthermore, CD36 was also induced in the absence of PXR, indicating that Aroclor 1260 had other targets related to CD36 induction and not restricted to PXR activation. Apart from lipogenic genes, the lipolytic gene PNPLA2 (adipose triglyceride lipase) was also evaluated. PNPLA2 is expressed primarily in the adipose tissue as well as in the liver (131); it is involved in breaking down triglycerides to free fatty acids and one of its transcriptional mediators is FOXO1 which is in turn controlled by many factors including sirtuins (SIRT1) and insulin $(132,133)$. Being a FOX01 target gene, we assumed that hepatic PNPLA2 expression pattern in our study would be similar to that of PEPCK-1. Aroclor 1260 induced PNPLA2 in CAR knockout mice, similar to PEPCK-1. However in PXR knockout mice, the basal levels of hepatic PNPLA2 were increased and Aroclor 1260 induction was not observed, suggesting that PXR rather than CAR may be dominant in the sequestration of FOXO1 under basal conditions. Moreover, PEPCK-1 basal levels were also higher in PXR knockout mice when compared to CAR knockout mice. 
Another distinct finding in this study was the regulation of PPARa target genes by CAR/PXR and Aroclor 1260 exposure (Fig. 3.14). Aroclor 1260 by itself increased PPARa expression in WT and PXR knockout mice but not in CAR knockout mice, suggesting that Aroclor 1260 exposure induced PPAR $\alpha$ but activated PXR negatively regulates this induction. Interestingly, Aroclor 1260 induced CPT1A, a PPARa target gene and rate limiting enzyme of mitochondrial fatty acid $\beta$-oxidation, in all three groups, and this was highest in the PXR knockout mice. Studies have shown the repression of CPT1A and other $\beta$ oxidation related genes with PXR and CAR activation and their enhanced expression in knockout models $(72,77,134)$. Apart from PPARa, CPT1A transcription is also mediated by the forkhead box protein A2 (FOXA2 ) and HNF4 $\alpha(135,136) . P X R$ is a known inhibitor of FOXA2, and hence its ablation increased FOXA2 basal expression and inducibility by Aroclor 1260 (105). FOXA2 activity is also positively regulated by low levels of insulin which was displayed by all the Aroclor 1260-exposed groups (137). Another plausible mechanism for increased CPT1A expression in the knockout mice could be the increased availability of the peroxisome proliferator-activated receptor gamma coactivator 1-alpha (PCG1 $\alpha$ ) for HNF4a transcriptional activity. Cyp4a10, another PPARa target and enzyme involved in peroxisomal fatty acid oxidation, was induced with Aroclor 1260 exposure in CAR knockout mice. This finding is consistent with studies by Ueda et al, demonstrating that CAR is a negative regulator of Cyp4a10 expression and hence knocking out CAR enhanced Cyp4a10 induction by Aroclor 1260 (134). 
Overall, our studies strongly indicated that CAR and PXR played a role in energy metabolism and validated previous findings that demonstrated xenobiotic receptor activation acts as an important factor in fatty liver disease and the metabolic syndrome (Fig. 3.16). In the current model, CAR and PXR activation appear to protect rather than augment Aroclor 1260-induced liver injury evident with worsened inflammation in CAR and PXR knockout mice. Moreover, the concept that CAR is an anti-obesity receptor and PXR is an obesity-promoting receptor may not reflect the complexity of Aroclor 1260 or environmental contaminant interaction with these transcription factors. It appears that both receptors are required for normal physiology and function and that they both portray similar if not identical outcomes on activation. Furthermore, our studies are consistent with previous observations on the effects of CAR and PXR in glucose and lipid metabolism. In contrast to the previous studies that employed model CAR and PXR ligands, this study used an environmental pollutant with multiple potential targets, which may also influence the end results of the study. These studies used knock out models to evaluate the distinct effects of these nuclear receptors in energy metabolism but it appears that Aroclor 1260 exposure also causes other effects such as compromised insulin levels. In conclusion, nuclear receptors CAR and PXR are not merely detoxification receptors but they also have an important role in inflammation and endobiotic metabolism as well; both CAR and PXR play an important protective role in liver injury caused by environmental pollutant, Aroclor 1260 . 


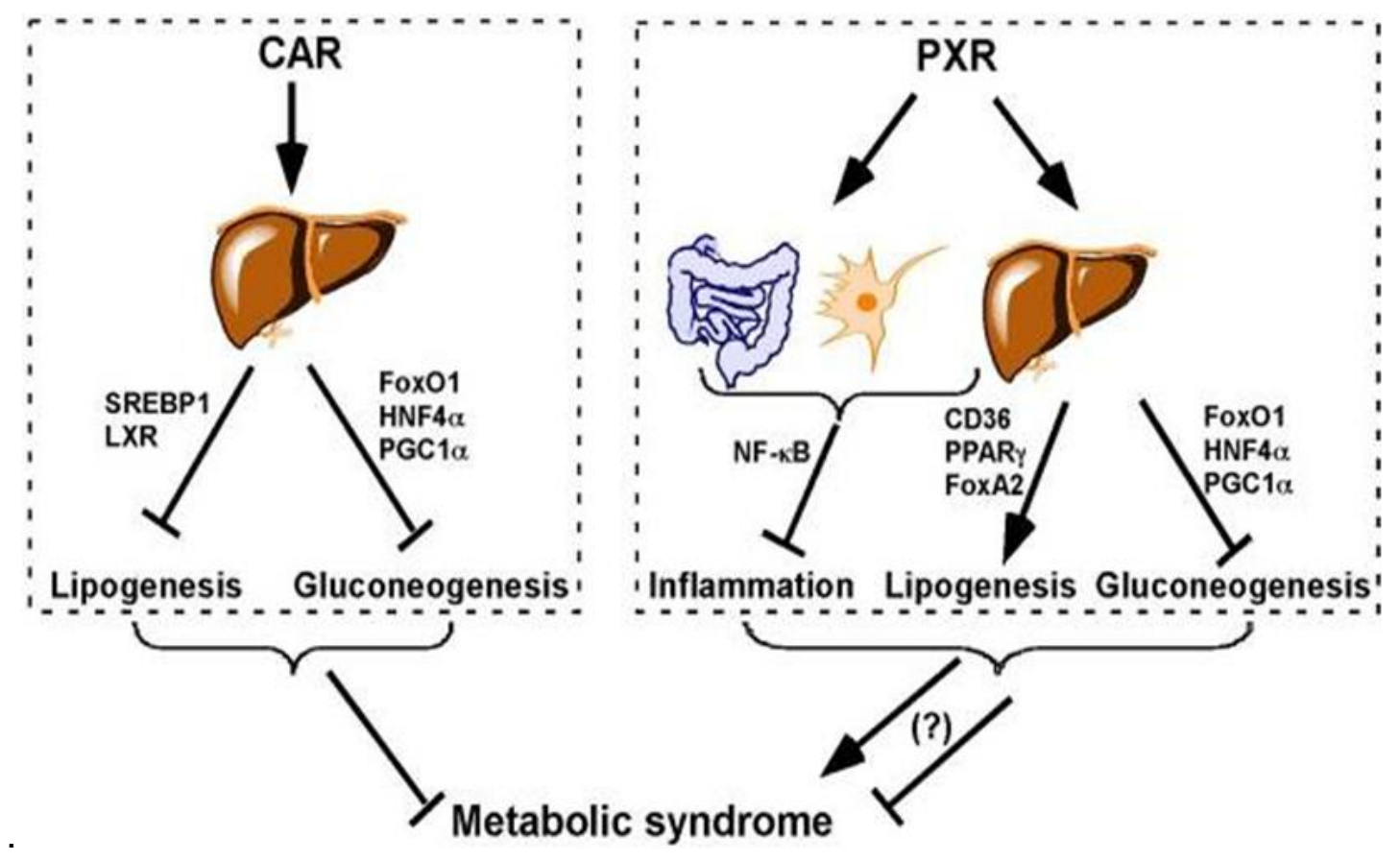

Figure 3. 16. Effects of CAR and PXR on hepatic energy metabolism.

A schematic diagram depicting the potential therapeutic role of CAR and PXR activation in the metabolic syndrome. Figure adapted from Gao, et al., Trends Pharmacol Sci, 33 (10), 2012. 


\section{CHAPTER 4}

\section{HUMAN RECEPTOR ACTIVATION BY AROCLOR 1260 AND INDIVIDUAL PCB CONGENERS \\ INTRODUCTION}

NAFLD and NASH were traditionally associated with the inappropriate over- or under-activation of nuclear receptors involved in endobiotic metabolism. These receptors include the LXR, farnesoid-X-receptor (FXR) and PPARs which regulate cholesterol, bile acid and lipid metabolism respectively $(58,59)$. Recent studies have implicated the role of hepatic receptors involved in xenobiotic detoxification, including PXR, CAR and the AhR in NAFLD/NASH. Although these receptors were initially thought to be involved only with detoxification and xenobiotic metabolism, over-activation or antagonism of these receptors may lead to metabolic disease states such as steatosis and obesity (60-62).

Historically, PCB toxicity has been linked to cancer, endocrine disruption and impaired cognitive development, but recent studies have shown that chronic exposure to these environmental pollutants can result in metabolic disorders associated with NAFLD, including obesity, insulin resistance/diabetes and the metabolic syndrome $(41,102)$. Additionally, rodent studies have correlated PCB exposures with NAFLD, obesity and the metabolic syndrome suggesting the involvement of distinct nuclear receptors in PCB-mediated toxicity (48). It 
remains unclear if the liver disease caused by PCB exposure is due to the direct involvement of receptors that regulate endobiotic metabolism such as $L X R$ and FXR or if the disease process is linked to the activation/inhibition of xenobiotic receptors such as PXR and CAR.

We hypothesized that PCBs may exert some of their toxic effects, such as NAFLD, by either interacting directly with the endobiotic nuclear receptors (LXR, FXR, PPARs) or through interaction with xenobiotic receptors that cross-talk with endobiotic receptors to otherwise modify their respective interactions with DNA response elements. Moreover, the interaction between Aroclor 1260 and these receptors that are implicated in NAFLD have never been tested. The purpose of this study is to evaluate the receptor agonism/antagonism by the PCB mixture, Aroclor 1260, and selected PCB congeners that are highly represented in this mixture. For the purpose of the studies, we collaborated with Dr. CJ Omiecinski from Penn State University to study PCB activation of the human CAR variants using COS-1 cells.

The study demonstrated selective activation by Aroclor 1260 and selected PCB congeners on human AhR, PXR and CAR, and inhibition of PPARa. We postulate that hepatic transcription factor activation is part of the mode of action of these organo-chlorine pollutants. 


\section{MATERIALS AND METHODS}

\section{Materials}

Aroclor 1260 was purchased from AccuStandard (New Haven, CN, USA) and PCB congeners were obtained from Ultra Scientific (North Kingstown, RI, USA). T0901317 (N-(2,2,2-trifluoroethyl)-N-[4-[2,2,2-trifluoro-1-hydroxy-1(trifluoromethyl)ethyl]phenyl]-benzenesulfonamide), GW3965 (2-(3-(3-((2-chloro3-(trifluoromethyl)benzyl)(2,2-diphenylethyl)amino)propoxy)phenyl) acetic acid hydrochloride), GW4064 (3-[2-[2-chloro-4-[[3-(2,6-dichlorophenyl)-5-(1methylethyl)-4-isoxazolyl]methoxy]phenyl]ethenyl] benzoic acid) and pioglitazone were obtained from Tocris Bioscience (Bristol, UK). Dimethyl sulfoxide (DMSO) was acquired from Fisher BioReagents (Thermo Fisher Scientific, Pittsburg, PA, USA) while 6-(4-chlorophenyl)imidazo[2,1-b][1,3]thiazole-5-carbaldehyde-O-(3,4dichlorobenzyl)oxime (CITCO), rifampicin (RIF), benz[a]anthracene (BA) and 3(4, 5-Dimethylthiazol-2-yl)-2, 5-diphenyltetrazolium bromide (MTT) were from Sigma Aldrich (St. Louis, MO, USA). Restriction endonucleases and T4 DNA ligase were purchased from New England BioLabs (Ipswich, MA). Lipofectamine and Opti-MEM were obtained from Life Technologies Inc (Carlsbad, CA, USA). Oligonucleotides were purchased from Integrated DNA Technologies (Coralville, IA, USA).

\section{Plasmid construction}

The reporter plasmids for human (h) PXR (pGL3-DR4-Luc), hFXR (pGL3IR1-Luc) and hPPARa (pGL3-DR1-Luc) were constructed by using two copies of a direct repeat 4 (DR4), an inverted repeat 1 (IR1) and a direct repeat 1(DR1) 
response element (RE) respectively. The top strand oligonucleotide was 5' AGAGTTCATGAGAGTTCATGAGAGTTCATGAGAGTTCATG 3' for pGL3-DR4Luc, 5' AGAGGTCATTGACCTTTAGAGGTCATTGACCTTT 3' for pGL3-IR1-Luc and 5' AACTAGGTCAAAGGTCAAACTAGGTCAAAGGTCAAA 3' for pGL3-DR1Luc. Both the bottom complementary strands had Kpn1 and Xho1 overhangs at the 5' and 3' positions respectively. The oligonucleotides were annealed and inserted into Xho1 and Kpn1 restriction sites in the polycloning region of a modified version of pGL3 promoter vector (Promega, Madison, WI, USA). Reporter plasmid for AhR (pXRE-SV40-Luc) was synthesized using the oligonucleotide 5' TCAGGCATGTTGCGTGCATCCCTGAGGCCAGCC 3' inserted into the EcoR1 site of a modified version of pGL3 promoter vector. Expression vectors pSG5-hLXRa, pSG5-hPXR and pSG5-hFXR and reporter plasmid pTK-LXRE-Luc were a generous gift from John Y. Chiang (Department of Integrative Medical Sciences, Northeast Ohio Medical University). Expression vectors pCMV6-hPPAR $\alpha$, pCMV6-hPPARy and pCMV6-hCAR (CAR2) were purchased from Origene (Rockville, MD, USA). The vectors, pTracer CMV2hCAR1, pTracer CMV2-hCAR2, pcDNA 3.1-RXRa, and pGL3- basic/TK CYP2B6-dervied XREM/PBREM were described previously (138). pTracer CMV2-CAR3 was also reported previously (139). pRL-CMV, the expression plasmid encoding Renilla luciferase, was purchased from Promega (Madison, WI, USA) and used in the Dual-Glo (Promega) assay system according to the manufacturer's protocol to normalize for transfection efficiencies in cultured cells. 
Prior to transfection, plasmids were prepared using the Qiagen Plasmid Plus Midi Kit (Qiagen, Valencia, CA, USA).

\section{Cell culture}

HepG2 cells: The human hepatoma-derived cell line (HepG2) was obtained from the American Type Culture Collection (ATCC, Manassas, MD, USA). Cells were grown in Dulbecco's modified Eagle's medium (DMEM, HyClone Laboratories Inc, Thermofisher, Waltham, MA, USA) supplemented with $10 \%$ fetal bovine serum (FBS) and 1\% antimycotic/antibiotic solution (Mediatech, Manassas, VA, USA). The cells were incubated in a $5 \%$ carbon dioxide atmosphere and $95 \%$ humidity at $37^{\circ} \mathrm{C}$ and subcultured every 2 days.

COS-1 cells: COS-1 cells (Simian virus-40-transformed African green monkey kidney cells) were obtained from the ATCC and maintained in DMEM plus GlutaMAX-I with 10\% FBS,10mM 4-(2-hydroxyethyl)-1-

piperazineethanesulfonic acid (HEPES), 1mM sodium pyruvate, 13 non-essential amino acids, and $1 \%$ penicillin/streptomycin. COS-1 cells were cultured at $37^{\circ} \mathrm{C}$ in a humidified atmosphere containing $5 \%$ carbon dioxide. All cell culture reagents were purchased from Life Technologies (Grand Island, NY, USA).

\section{Cell viability assay}

HepG2 cells were seeded in 96-well tissue culture plates at a seeding density of 10,000 cells per well. Cells were treated with graded concentrations of Aroclor 1260 made up as 500X stocks in DMSO. Controls received DMSO only. After $24 \mathrm{~h}$ incubation, MTT $(0.2 \mathrm{mg} / \mathrm{ml})$ was added to the cells and incubated for 3-4 $\mathrm{h}$. The media were removed from the plates by aspiration and formazan dye 
was liberated by adding $50 \mu \mathrm{L}$ DMSO. MTT, a yellow tetrazole, was reduced to an insoluble product formazan by mitochondrial reductases, indicative of cell viability. Presence of formazan, a purple precipitate, was determined spectrophotometrically at $540 \mathrm{~nm}$ using a Bio-Tek Synergy HT multi-mode micro plate reader (Winooski, VT, USA).

\section{Transfection}

HepG2 cells were plated in Thermo Scientific Nunc 24-well plates and transfected at $40-60 \%$ confluence. Unless otherwise specified, the transfection mix per well contained $150 \mathrm{ng} \beta$-galactosidase expression plasmid (pCMV- $\beta$, Stratagene, CA) as a transfection control, $50 \mathrm{ng}$ receptor expression plasmid and $150 \mathrm{ng}$ reporter plasmid. All cells were co-transfected by lipofection using Lipofectamine reagent according to the manufacturer's instructions and OptiMEM (reduced serum medium) as the transfecting medium. After 4 hour incubation, the medium was changed to DMEM supplemented with $10 \%$ FBS and $1 \%$ antimycotic/antibiotic solution and cells were allowed to recover overnight. DMEM supplemented with charcoal/dextran treated FBS (HyClone Laboratories Inc, Thermofisher, Waltham, MA, USA) was used for PPAR activation assays. Compounds of interest were then added to the cells $(n=4)$ and cells were incubated for $24 \mathrm{~h}$. DMSO was used as a carrier for all compounds (final concentration $<0.5 \%$ ). For COS-1 cells, all media components remained the same except that FBS was replaced with 10\% dextran/charcoal-treated FBS (HyClone, Logan, UT, USA). The details of the luciferase reporter assays were described previously (138-140). Briefly, approximately $1 \mathrm{~h}$ prior to transfection, 
cells were trypsinized and plated onto 48 -well plates $(\sim 50,000$ cells per well). For determination of transcriptional activity of the hCAR constructs, cells were transfected using Fugene 6 (Promega) according to the manufacturer's recommendations with a co-transfection plasmid mix consisting of $10 \mathrm{ng} \mathrm{pRL}-$ CMV (Renilla luciferase) for normalization, $25 \mathrm{ng}$ pcDNA 3.1-RXRa, $100 \mathrm{ng}$ of pGL3-basic/TK XREM/PBREM luciferase reporter plasmid, and $25 \mathrm{ng}$ of $\mathrm{pTracer}$ vectors containing the various CMV2-CAR expression constructs. Each condition was performed in quadruplicate. Aroclor 1260 and each of the PCBs were evaluated at $10 \mu \mathrm{M}$. DMSO was used as a solvent control. CITCO, $5 \mu \mathrm{M}$, was used as a positive control. Androstanol (ANDR), a known inverse agonist of human CAR, was used as a control for CAR1.

\section{Reporter assay}

HepG2 cells: Cells were washed twice with Phosphate Buffered Saline (1X), harvested using $50 \mu \mathrm{L}$ cell lysis buffer (Promega, Madison, WI, USA) and subjected to a single freeze-thaw event. For $\beta$-galactosidase assays, cell extracts $(5 \mu \mathrm{L})$, were incubated with chlorophenol red $\beta$-galactopyranoside (CPRG, Roche Diagnostics, Indianapolis, IN) at $37^{\circ} \mathrm{C}$ for $30-60$ minutes. The enzyme activity was determined spectrophotometrically at $595 \mathrm{~nm}$ using the BioTek Synergy HT multi-mode micro plate reader. Luciferase activity assays were performed on cell extracts $(5 \mu \mathrm{L})$ using the Luciferase Assay System (Promega). Luminescence was measured using the Orion $L$ micro plate luminometer (Berthold Detection Systems, Pforzheim, Germany) over a 10 second period. Receptor activation was measured by luciferase activity and results were 
normalized to the amount of $\beta$-galactosidase expressed. COS-1 cells: Luciferase assays were performed using the Dual-Glo Reporter Assay System (Promega) and a Veritas Microplate Luminometer (Turner Biosystems, Sunnyvale, CA, USA). Firefly (Photinus pyralis) luminescence data values were recorded for each replicate and normalized luciferase activities were then calculated by dividing the raw luciferase values by the Renilla luciferase signals to correct for any differences in transfection efficiency among the assay wells.

\section{Validation of receptor activation in primary human hepatocytes}

Human hepatocytes were obtained from BioreclamationIVT (Baltimore, MD, USA). Hepatocytes were thawed and plated in 12-well plates according to the supplier's protocols and the compounds of interest were added. The cells were incubated for $24 \mathrm{~h}$ and RNA was extracted using RNA STAT-60 protocol (Tel-test, Austin, TX, USA). RNA purity and quantity were assessed with the Nanodrop (ND-1000, Thermo Scientific, Wilmington, DE, USA) using the ND1000 V3.8.1 software. cDNA was synthesized from total RNA using the QuantiTect Reverse Transcription Kit (Qiagen, Valencia, CA, USA). Polymerase chain reaction (PCR) was performed on the Applied Biosystems StepOne Plus Real-Time PCR Systems using the Taqman Universal PCR Master Mix (Life Technologies, Carlsbad, CA, USA). Each PCR mix (20 $\mu \mathrm{L})$ contained: Taqman Universal PCR Master Mix (10 $\mu \mathrm{L}), 20 X$ Gene Expression Assay Mix (1 $\mu \mathrm{L})$, cDNA sample $(2 \mu \mathrm{L})$ and nuclease-free water $(7 \mu \mathrm{L})$. Primer sequences from Taqman Gene Expression Assays (Applied Biosystems, Foster City, CA, USA) were as follows: cytochrome P450s [CYP3A4 (Hs00604506_m1), CYP2B6 
(Hs04183483_g1), CYP1A1 (Hs01054797_g1)], CD36 (Hs01567185_m1), fatty acid synthase (FAS) (Hs01005622_m1), carnitine palmitoyl transferase 1A (CPT1A), (Hs00912671_m1), small heterodimeric partner (SHP) (Hs00222677_m1) and glyceraldehyde-3-phosphate dehydrogenase (GAPDH). Cycle conditions were maintained according to the Applied Biosystems guide. The levels of mRNA were normalized relative to the amount of GAPDH mRNA, and expression levels in DMSO-exposed cells were set at 1\%. Gene expression levels were calculated according to the $2^{-\Delta \Delta C t}$ method (92).

\section{Statistical analysis}

Statistical analyses were performed using GraphPad Prism version 5.01 (San Diego, CA, USA). In general for all assays, data are expressed as means \pm SEM. Quantitative data for two group comparisons were assessed using an unpaired t-test. Multiple group data were examined by one way analysis of variance followed by the Dunnett's post hoc test to compare all groups to the control sample. Multiplicity adjusted $p$ values are reported in the Results section. $P<0.05$ was considered statistically significant. 


\section{RESULTS}

\section{Cell viability assay for Aroclor 1260}

MTT assays were performed to determine the optimal concentration for Aroclor 1260 that does not cause toxicity in HepG2 cell culture experiments. Cells were exposed to this PCB mixture at concentrations ranging from 1.25 $250 \mu \mathrm{g} / \mathrm{mL}$. The optical density for formazan, representative of cell viability, was plotted against Aroclor 1260 concentrations. The toxicity threshold (concentration that caused $50 \%$ cell death) was determined to be $26.0 \pm 3.7 \mu \mathrm{g} / \mathrm{mL}$ (Fig. 4.1). Concentrations of Aroclor 1260 at 5, 10, 15 and $20 \mu \mathrm{g} / \mathrm{mL}$ were selected for subsequent experiments. 


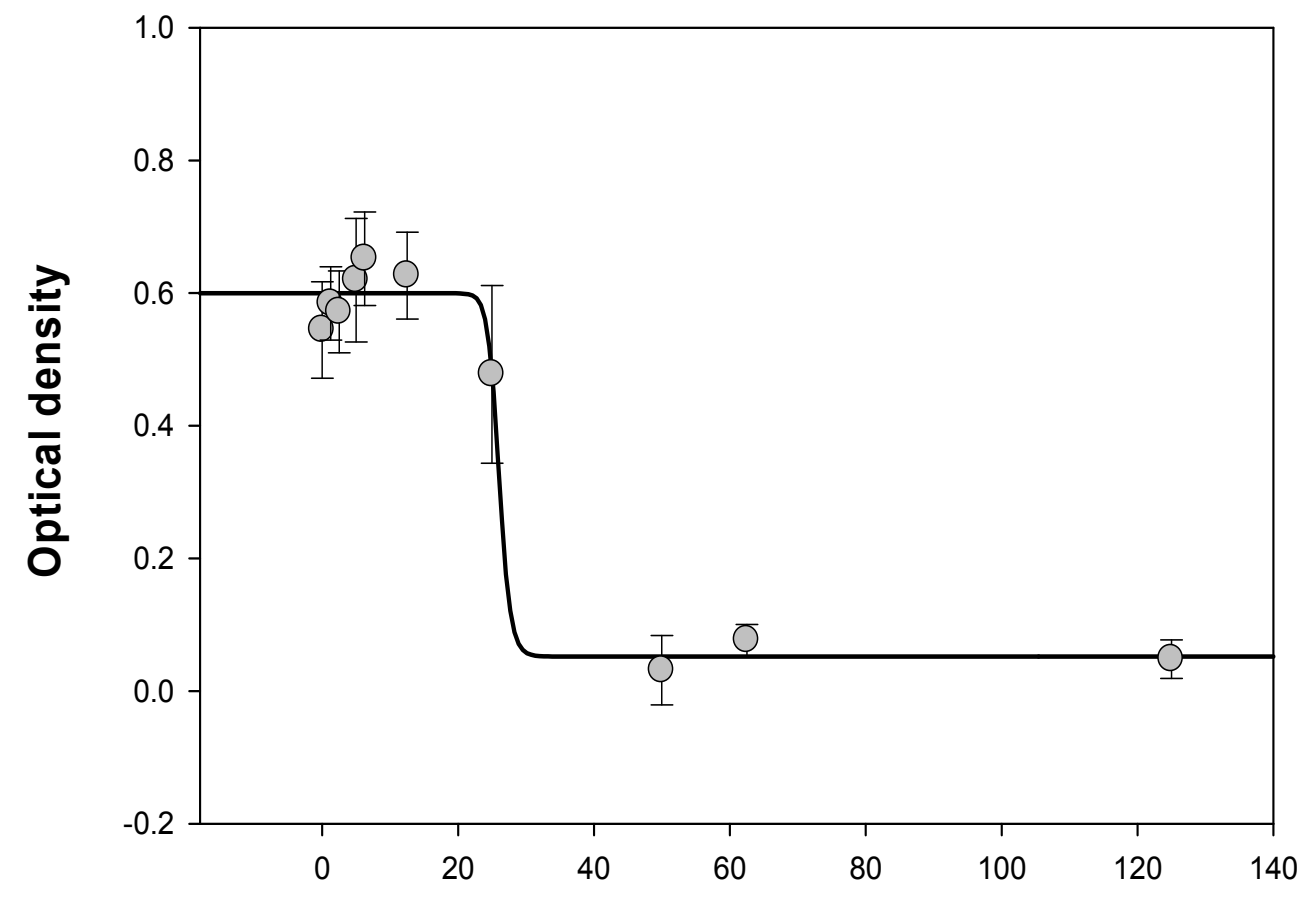

Aroclor $1260(\mu \mathrm{g} / \mathrm{mL})$

Figure 4.1. Cell viability assay for Aroclor 1260.

HepG2 cells were exposed to Aroclor 1260 at concentrations ranging from

$1.25-250 \mu \mathrm{g} / \mathrm{mL}$ and the optical density for formazan was measured. 


\section{Aroclor 1260 activation of the human aryl hydrocarbon receptor}

Coplanar PCBs (PCBs 126 and 77) activate AhR in rodents and hence are classified as "dioxin-like" PCBs. Coplanar congeners comprise only $\sim 1 \%$ of the total composition in Aroclor 1260. HepG2 cells, co-transfected with pXRESV40-Luc, were exposed to various concentrations of Aroclor 1260 for $24 \mathrm{~h}$ and the normalized luciferase activity was measured. As anticipated, AhR was activated by its polycyclic aromatic hydrocarbon ligand BA $(10 \mu \mathrm{M})$, and Aroclor 1260 exposure resulted in a significant increase in luciferase activity (3.5-fold at $20 \mu \mathrm{g} / \mathrm{mL}, p<0.0001$ ) compared to DMSO-exposed cells (Fig. 4.2 A). However, this fold induction was relatively low when compared to that of BA. These results indicate that Aroclor 1260 has a weak AhR agonistic activity that is likely due to the presence of coplanar congeners at lower concentrations in the mixture. AhR activation by BA was assessed in the presence of increasing concentrations of Aroclor 1260 to evaluate for any potentiation or antagonism effects. Cells coexposed to BA and Aroclor 1260 showed no difference vs. BA-exposed cells only (Fig. 4.2 B).

Next, we selected ten non-coplanar congeners that are more highly represented in Aroclor 1260 mixture ( $\geq 1 \%)$ as well as two coplanar congeners (PCBs 126 and 118) and tested the ability of these compounds to individually activate AhR at a concentration of $10 \mu \mathrm{M}$. Although PCB 126 (no ortho) constitutes $\sim 0.002 \%$ of the total PCB composition in Aroclor 1260 , it was selected for this study because it is a good AhR activator (141). PCB 118 (monoortho) was chosen because it was one of the coplanar congeners with the 
highest composition in Aroclor 1260 (0.48\%). Among the selected congeners, coplanar PCB 126 and non-coplanar PCB 138 significantly induced luciferase activity (17-fold, $p<0.0001$ and 4-fold, $p=0.0006$ respectively), while coplanar PCB 118 did not induce luciferase activity (1.2-fold) compared to DMSO-exposed cells (Fig. 4.2 C). Thus it appeared that PCB 118 did not activate AhR at the concentration used, unlike PCB 126. Besides, AhR activation was not restricted to only coplanar congeners because PCB 138 modestly activated AhR. 

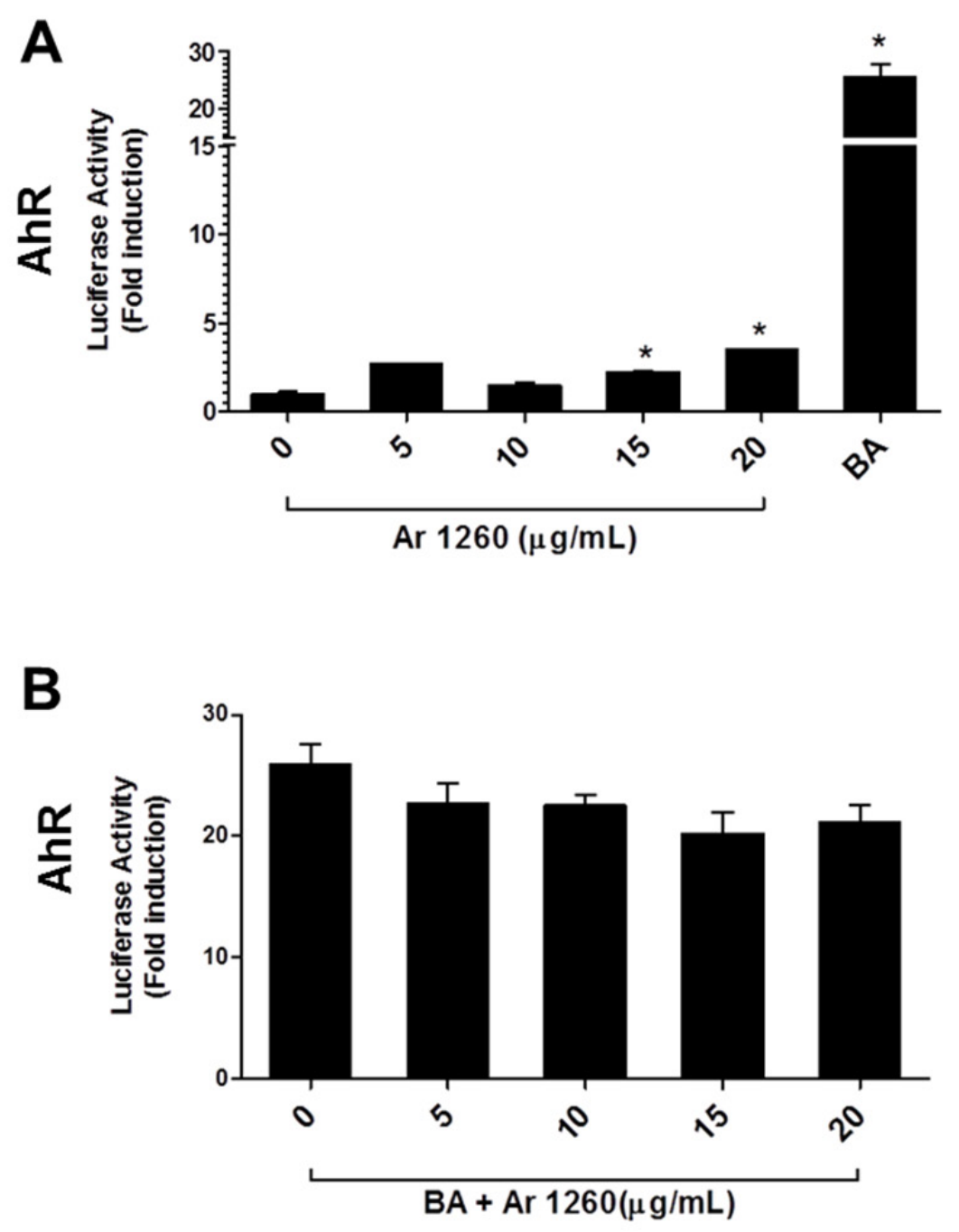

C

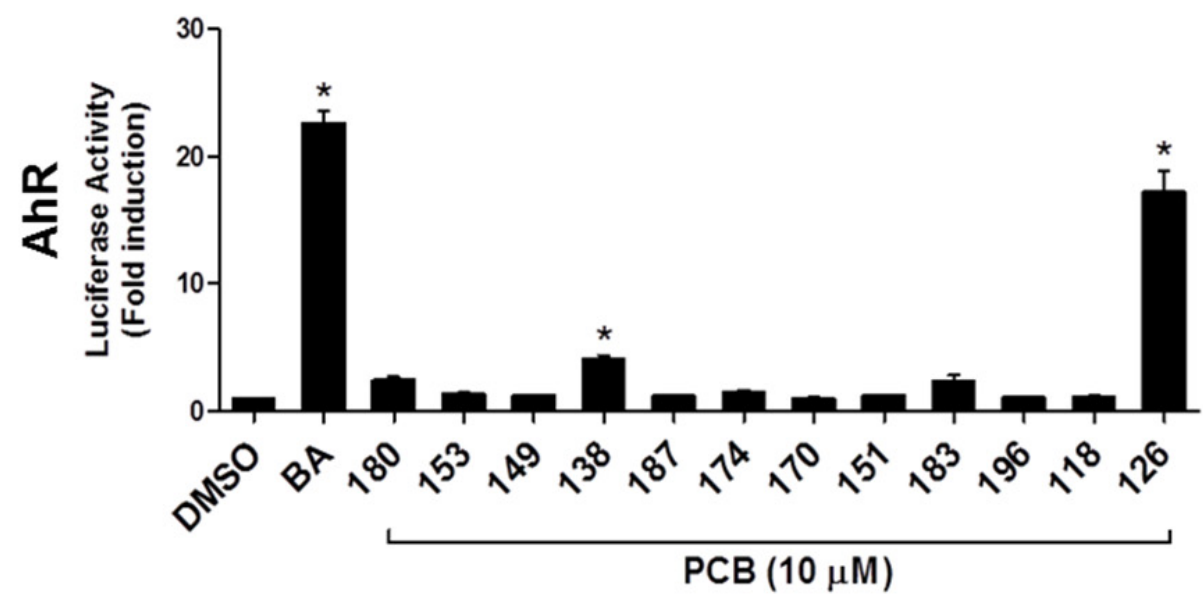


Figure 4.2. Aroclor 1260 activation of the human AhR.

HepG2 cells were transiently transfected with the reporter plasmid pXRESV40-Luc. Benz[a]anthracene (10 $\mu \mathrm{M}, \mathrm{BA})$ was used as a positive control. (A) Cells were exposed to Aroclor 1260 at $0,5,10,15$ and $20 \mu \mathrm{g} / \mathrm{mL}$ and luciferase induction was normalized and compared to DMSO-exposed cells $(0 \mu \mathrm{g} / \mathrm{mL}$ Aroclor 1260). (B) Cells were exposed to $10 \mu \mathrm{M}$ BA or BA plus Aroclor 1260 at 0 , $5,10,15$ and $20 \mu \mathrm{g} / \mathrm{mL}$. The luciferase induction was normalized to that of cells exposed only to DMSO solvent carrier (as in A, not shown). Luciferase activity in cells exposed to BA and Aroclor 1260 was compared to that of BA-exposed cells. (C) Cells were exposed to selected PCB congeners $(10 \mu \mathrm{M})$ present in Aroclor 1260 and the induction was compared to DMSO-exposed cells. Data were normalized to luciferase activity in cells exposed only to DMSO and are expressed as mean \pm SEM, $n=4,{ }^{*} p<0.05$. Figure adapted from Wahlang et al., Toxicol Sci, 140 (2), 2014. 


\section{Aroclor 1260 activation of the human pregnane xenobiotic receptor}

Non-coplanar PCBs, including PCBs 153 and 196, have been predicted to activate the nuclear receptors PXR and CAR, and these PCBs are often referred to as "phenobarbital-like". Apart from regulating xenobiotic metabolism, PXR activation is implicated in weight gain and obesity $(60,142)$. Moreover, since PXR and CAR do share similar ligands to an extent, it is therefore likely that Aroclor 1260, being largely composed of non-coplanar PCBs, will activate PXR.

HepG2 cells, co-transfected with pSG5-hPXR and pGL3-DR4-Luc were exposed to various concentrations of Aroclor 1260 for 24 h. RIF (10 $\mu \mathrm{M})$, a PXR ligand, was used as a positive control. RIF activated PXR-driven luciferase activity (2.6-fold, $p<0.0001)$ whereas Aroclor 1260 activated the receptor in a concentration-dependent manner with a $\mathrm{Km}$ value of $8.75 \mu \mathrm{g} / \mathrm{mL}$ (Fig. $4.3 \mathrm{~A}$ ). Cells exposed to Aroclor 1260 at $5 \mu \mathrm{g} / \mathrm{mL}$ showed no significant induction in luciferase activity as compared to cells exposed to DMSO only. However, the induction was significant at concentrations of $10(1.9$-fold, $p=0.0024), 15(2.1$ fold, $p=0.0001)$ and $20(2.7$-fold, $p<0.0001) \mu \mathrm{g} / \mathrm{mL}$. When transfected cells were exposed to both RIF and Aroclor 1260 ( $5 \mu \mathrm{g} / \mathrm{mL}$ and above) simultaneously, there was a slightly higher induction ( 18\%) compared to RIF-exposed cells alone (Fig. 4.3 B) but this was not significant. These data suggest that Aroclor 1260 activated the PXR reporter system at concentrations of $10 \mu \mathrm{g} / \mathrm{mL}$ and higher and that the mixture did not significantly potentiate or antagonize PXR activation by the receptor ligand, RIF. We then evaluated the ability of selected PCB congeners to activate PXR (Fig. 4.3 C). Of the congeners tested, non- 
coplanar PCBs that significantly increased luciferase activity at $10 \mu \mathrm{M}$ included PCBs 149 (2.1-fold, $p$ <0.0001), 138 (1.7-fold, $p=0.0006$ ), 187 (2.0-fold, $p$ <0.0001), 174 (2.3-fold, $p<0.0001$ ), 151 (1.6-fold, $p=0.0062$ ), 183 (1.6-fold, $p$ $=0.0067)$ and $196(1.6$-fold, $p=0.0036)$. Notably, these congeners in total represent more than $30 \%$ of the Aroclor 1260 mixture by mass. Interestingly, PCB 126, a well-known AhR ligand and a coplanar congener also activated human PXR (1.9-fold, $p<0.0001)$, indicating that a PCB's non-coplanar structure is not solely required for this receptor's activation. 

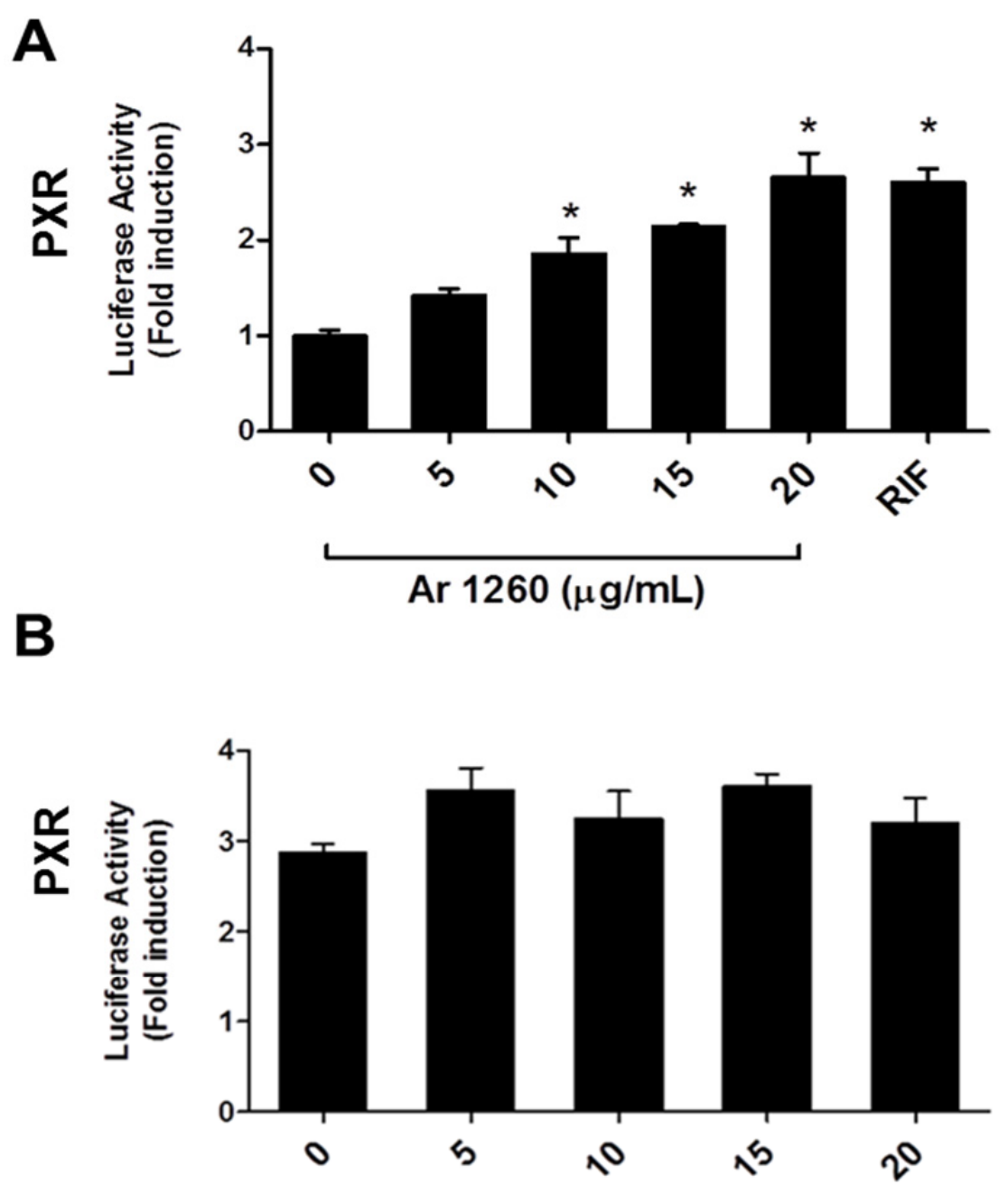

$$
\operatorname{RIF}+\operatorname{Ar} 1260(\mu \mathrm{g} / \mathrm{mL})
$$

C

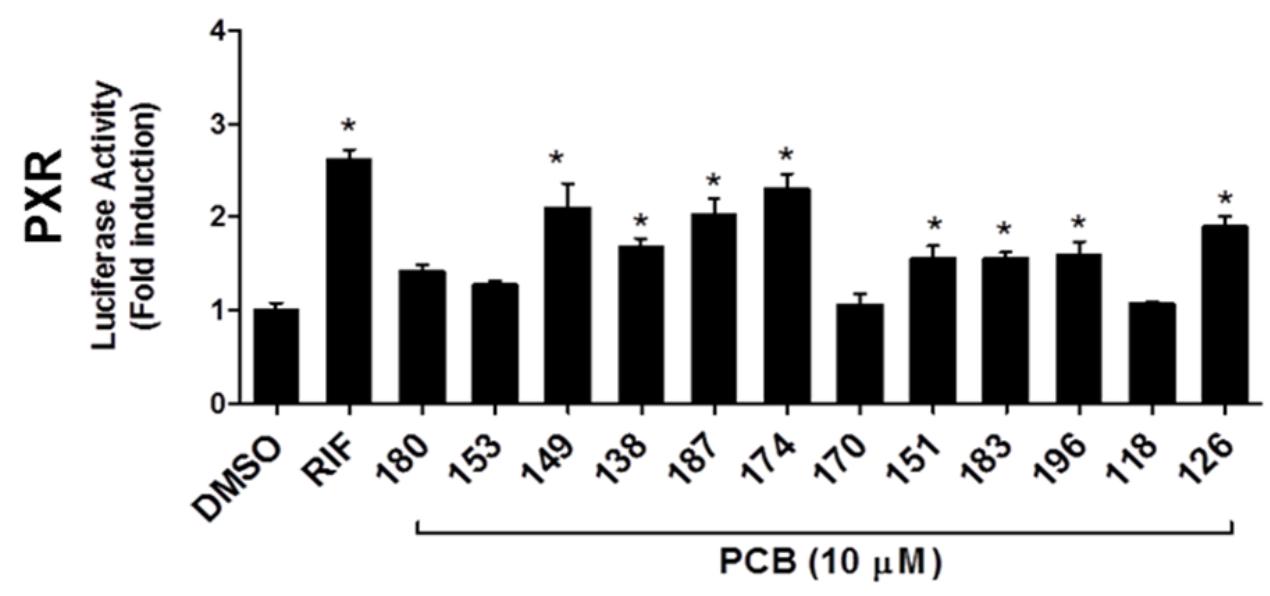




\section{Figure 4.3. Aroclor 1260 activation of the human PXR.}

HepG2 cells were transiently transfected with the expression plasmid pSG5-hPXR and reporter plasmid pGL3-DR4-Luc. Rifampicin (10 $\mu \mathrm{M}, \mathrm{RIF})$ was used as a positive control. (A) Cells were exposed to Aroclor 1260 at 0, 5, 10, 15 and $20 \mu \mathrm{g} / \mathrm{mL}$ and luciferase induction was normalized and compared to DMSOexposed cells $(0 \mu \mathrm{g} / \mathrm{mL}$ Aroclor 1260$)$. (B) Cells were exposed to $10 \mu \mathrm{M}$ RIF or RIF plus Aroclor 1260 at $0,5,10,15$ and $20 \mu \mathrm{g} / \mathrm{mL}$. The luciferase induction was normalized to that of cells exposed only to DMSO solvent carrier (as in A, not shown). Luciferase activity in cells exposed to RIF and Aroclor 1260 was compared to that of RIF-exposed cells. (C) Cells were exposed to selected PCB congeners $(10 \mu \mathrm{M})$ present in Aroclor 1260 and the induction was compared to DMSO-exposed cells. Data were normalized to luciferase activity in cells exposed only to DMSO and are expressed as mean \pm SEM, $n=4,{ }^{*} p<0.05$. Figure adapted from Wahlang et al., Toxicol Sci, 140 (2), 2014. 


\section{Aroclor 1260 activation of the human constitutive androstane receptor}

In these experiments, HepG2 cells were co-transfected with pCMV6hCAR (CAR2) and pGL3-DR4-Luc and the ability of Aroclor 1260 to transactivate CAR2 was evaluated. CAR 2 is a human splice variant of the CAR1 reference form of the receptor, but unlike the reference form, CAR2 is not constitutively active; rather, it is a ligand activated receptor (138). Although somewhat modest, the human CAR agonist CITCO and Aroclor $1260(10 \mu \mathrm{g} / \mathrm{mL})$ significantly activated CAR2 (2.8-fold, $p<0.0001$ and 1.6-fold, $p=0.0240$ respectively) (Fig. 4.4 A). However, increasing the concentration of Aroclor 1260 did not lead to further CAR2 activation (1.1-fold at $15 \mu \mathrm{g} / \mathrm{mL}$ and 1.4-fold at $20 \mu \mathrm{g} / \mathrm{mL}$ respectively). Rather, this PCB mixture appeared to antagonize CAR2 activation by CITCO (Fig. 4.4 B). Aroclor 1260 , at $20 \mu \mathrm{g} / \mathrm{mL}$, significantly reduced the induction produced by CITCO by $51 \%(p=0.0314)$. These results suggested that Aroclor 1260 may bind to CAR2 and either activate CAR2 or inhibit CAR2 activation by CITCO in a concentration-dependent manner. Interestingly, two of the selected congeners, PCBs $187(p=0.0042)$ and $126(p=0.0031)$, increased luciferase induction significantly at $10 \mu \mathrm{M}$ concentration (Fig. 4.4 C), indicating that some of the PCB congeners in Aroclor 1260 may be CAR2 agonists.

Given the apparent impact of these PCB congeners on human CAR, we designed a complimentary series of assays to further corroborate and better characterize these effects using the primate-derived cell line, COS-1. These results are presented in Figure 4.5. Due to the high constitutive activity of CAR1, any ligand interactions with the receptor tend to be masked. In these respects, it 
is notable that PCB 174 significantly inhibited the constitutive activity of CAR1 ( $p$ $<0.05)$, though not as remarkably as ANDR $(p<0.001)$, a known CAR inverse agonist (Fig. 4.5 A). Although the level of activation of CAR2 did not reach the level of the CITCO positive control (5.4-fold, $p<0.001)$, Aroclor 1260 as well as several other PCB congeners demonstrated significant CAR2 activation in the COS-1 cell assays (Fig. 4.5 B). Exhibiting a fold change of $2.4(p<0.001)$, PCB 126 elicited the greatest response - even greater than Aroclor $1260(1.7$-fold, $p$ $<0.05)$. Four additional congeners also activated CAR2: $180(1.7$-fold, $p<0.05)$, 149 (1.9-fold, $p<0.01$ ), 187 (2.0-fold, $p<0.001)$ and 196 (1.8-fold, $p<0.01$ ). CAR3, another ligand activated human splice variant, which likely maintains a conserved ligand binding pocket with CAR1, was also tested and exhibited significant activation effects with Aroclor 1260 (7.6-fold, $p<0.001)$ (Fig. 4.5 C). Several other PCB congeners also demonstrated significant CAR3 activation including PCB 187 (17.1-fold, $p<0.001$; comparable to CITCO at 18.5-fold, $p$ <0.001), PCB 153 (6.0-fold, $p$ <0.01), PCB 149 (10.6-fold, $p<0.001$ ), PCB 138 (8.3-fold, $p<0.001$ ), PCB 151 (5.5-fold, $p<0.01$ ), and PCB183 (15.1-fold, $p$ $<0.001)$. 


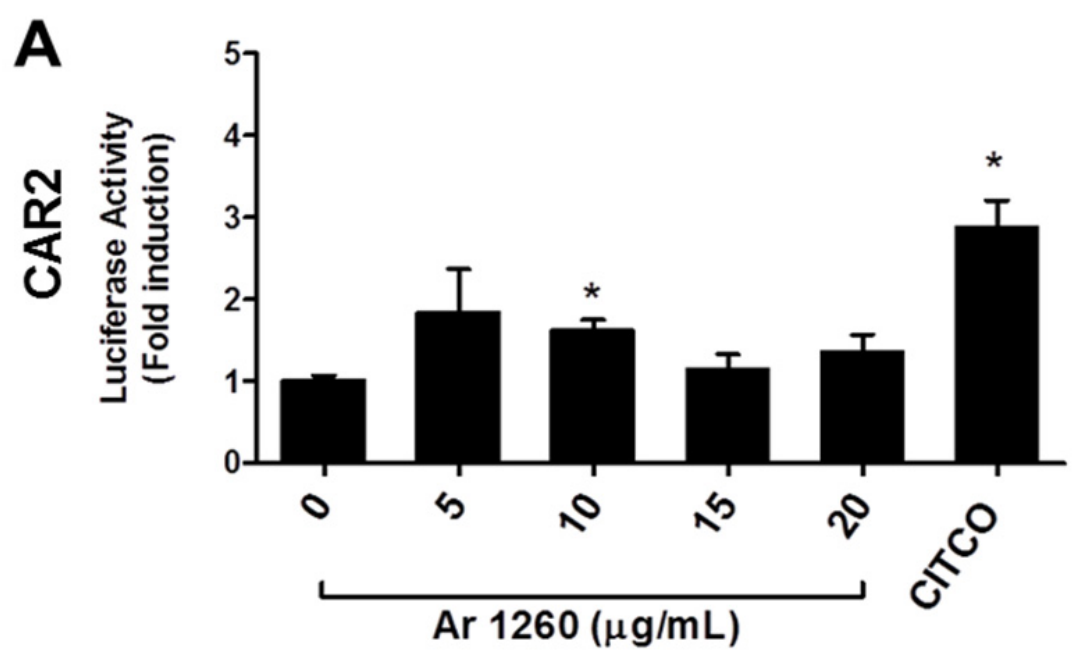

B

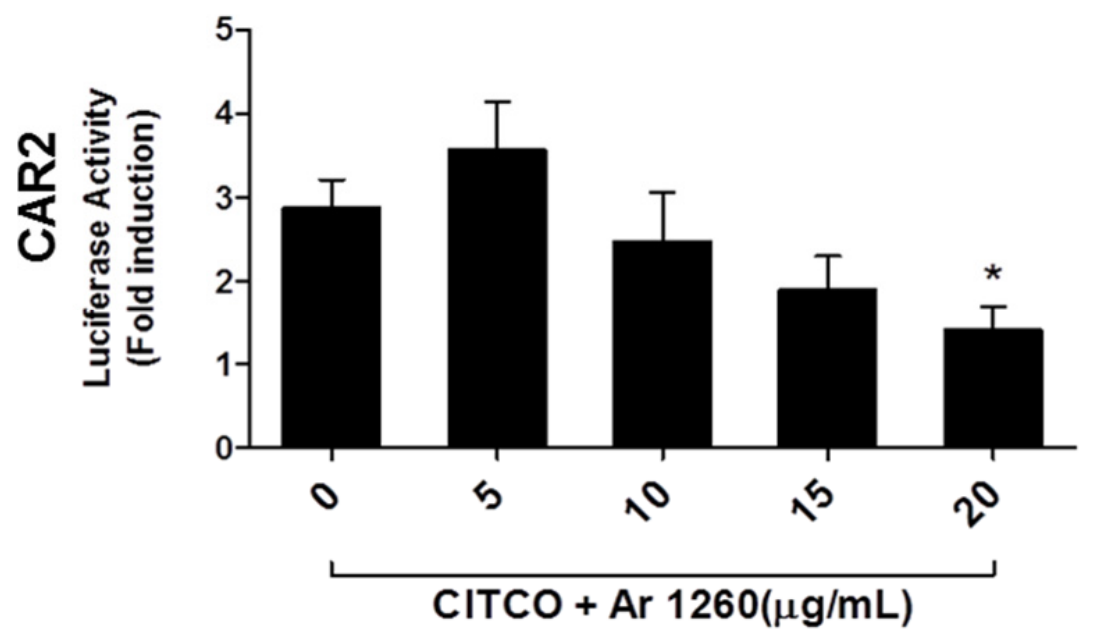

C

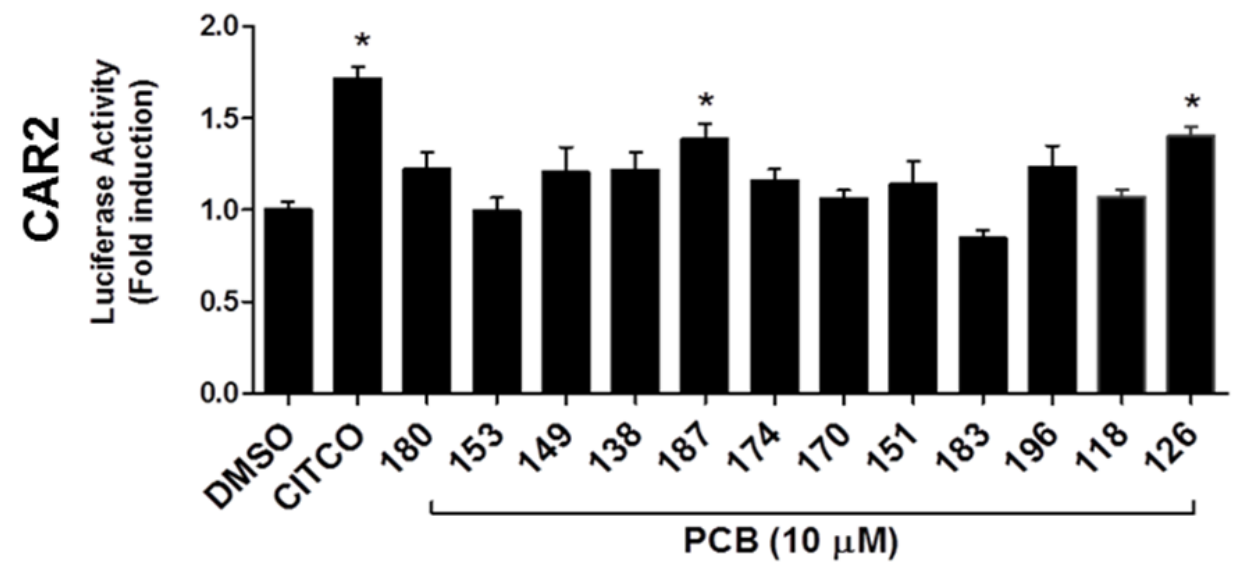




\section{Figure 4.4. Aroclor 1260 activation of the human CAR2 transcript.}

HepG2 cells were transiently transfected with the expression plasmid pCMV6-hCAR (CAR2) and reporter plasmid pGL3-DR4-Luc. CITCO $(10 \mu \mathrm{M})$ was used as a positive control. (A) Cells were exposed to Aroclor 1260 at 0, 5, 10, 15 and $20 \mu \mathrm{g} / \mathrm{mL}$ and luciferase induction was normalized and compared to DMSOexposed cells ( $0 \mu \mathrm{g} / \mathrm{mL}$ Aroclor 1260). (B) Cells were exposed to $10 \mu \mathrm{M}$ CITCO or CITCO plus Aroclor 1260 at 0, 5, 10, 15 and $20 \mu \mathrm{g} / \mathrm{mL}$. The luciferase induction was normalized to that of cells exposed only to DMSO solvent carrier (as in A, not shown). Luciferase activity in cells exposed to CITCO and Aroclor 1260 was compared to that of CITCO-exposed cells. (C) Cells were exposed to selected PCB congeners $(10 \mu \mathrm{M})$ present in Aroclor 1260 and the induction was compared to DMSO-exposed cells. Data were normalized to luciferase activity in cells exposed only to DMSO and are expressed as mean \pm SEM, $n=4,{ }^{*} p<0.05$. Figure adapted from Wahlang et al., Toxicol Sci, 140 (2), 2014. 

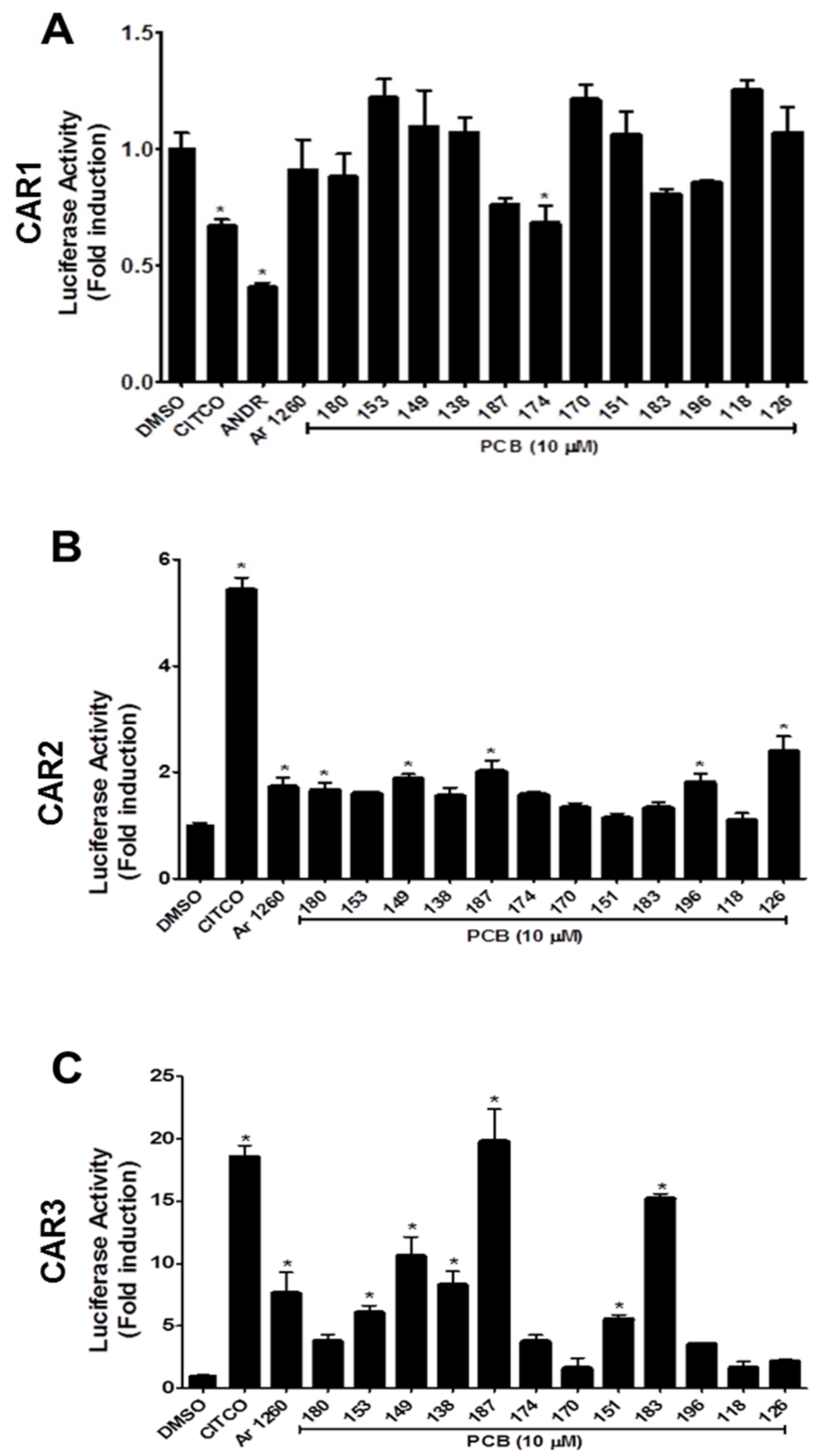
Figure 4.5. Aroclor 1260 activation of the human CAR variant transcripts in COS-1 cells.

COS-1 cells were transiently transfected with expression plasmid pTracerCMV2 containing either (A) CAR1, (B) CAR2 or (C) CAR3 transcripts, together with the reporter plasmid, pGL3-2B6XREM/PBREM-TKLuc. Cells were exposed to selected PCB congeners $(10 \mu \mathrm{M})$ present in Aroclor 1260 and the induction was compared to DMSO-exposed cells. CITCO $(5 \mu \mathrm{M})$ was used as a positive control. ANDR $(10 \mu \mathrm{M})$, a CAR inverse agonist, was used as a negative control for CAR1. Data were normalized to luciferase activity in cells exposed only to DMSO and are expressed as mean \pm SEM, $n=4,{ }^{*} p<0.05$. Figure adapted from Wahlang et al., Toxicol Sci, 140 (2), 2014. 


\section{Effect of Aroclor 1260 on the human liver-X-receptor alpha}

The human LXRa is a subtype of LXR that is expressed in the liver and its under- or over-activation eventually leads to steatosis (143). We therefore hypothesized that Aroclor 1260 may activate LXRa and this activation may subsequently promote hepatic steatosis and NAFLD. HepG2 cells, co-transfected with pSG5-hLXR $\alpha$ and pTK-LXRE-Luc, were exposed to various concentrations of Aroclor 1260 for $24 \mathrm{~h}$ and the normalized luciferase activity measured. T0901317, a synthetic LXRa ligand was used as a positive control. Compared to the fold induction by T0901317 (100 nM), Aroclor 1260 did not activate LXRa nor antagonize LXR $\alpha$ activation by T0901317 at any concentration tested (Fig. 4.6 $A \& B)$, thus indicating that this PCB mixture is neither an agonist nor antagonist of the nuclear receptor $L X R \alpha$. 


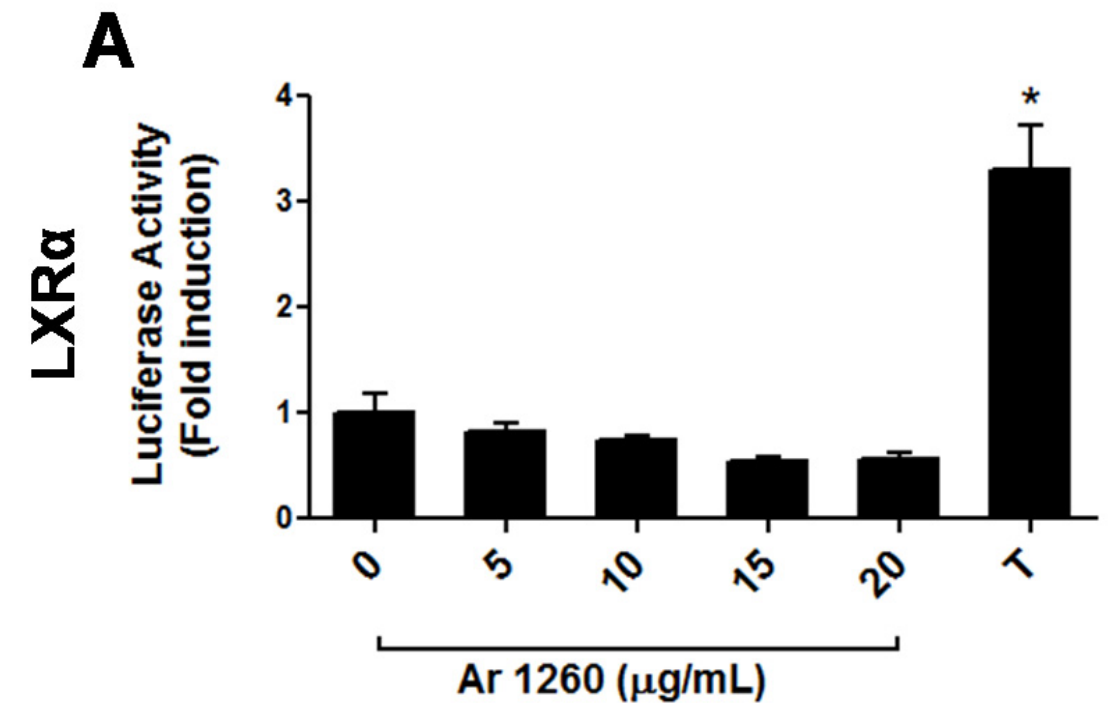

B

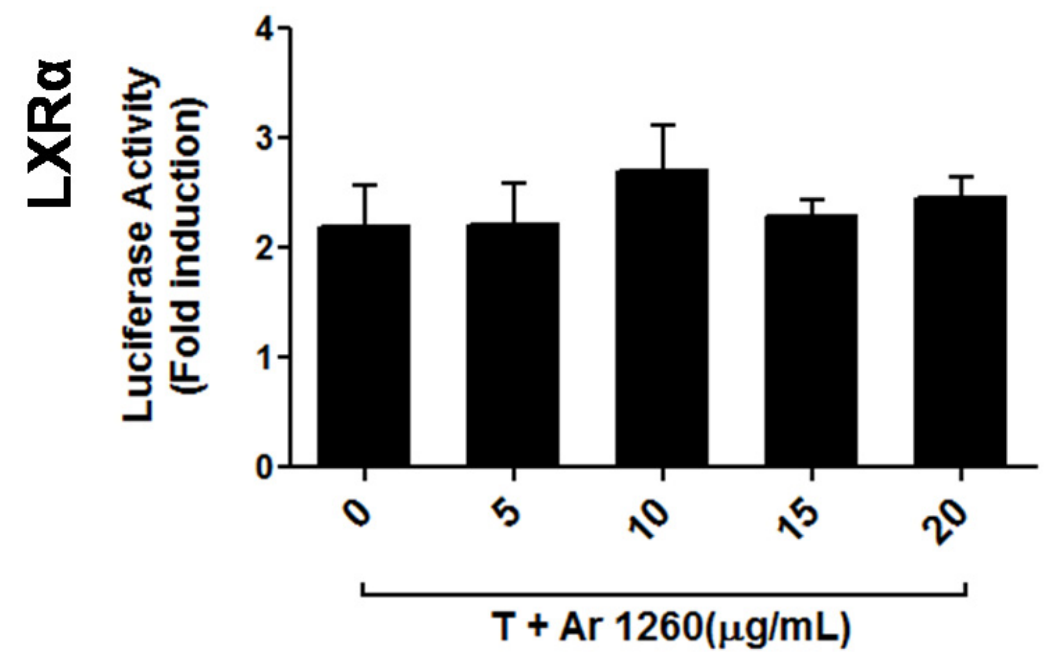


Figure 4.6. Aroclor 1260 activation of the human LXRa.

HepG2 cells were transiently transfected with the expression plasmid pSG5-hLXRa and reporter plasmid pTK-LXRE-Luc. T0901317 (100nM, T) was used as a positive control. (A) Cells were exposed to Aroclor 1260 at 0, 5, 10, 15 and $20 \mu \mathrm{g} / \mathrm{mL}$ and luciferase induction was normalized and compared to DMSOexposed cells $(0 \mu \mathrm{g} / \mathrm{mL}$ Aroclor 1260$)$. (B) Cells were exposed to $100 \mathrm{nM} \mathrm{T}$ or $\mathrm{T}$ plus Aroclor 1260 at $0,5,10,15$ and $20 \mu \mathrm{g} / \mathrm{mL}$. Luciferase activity in cells exposed to T and Aroclor 1260 was compared to that of T-exposed cells. Data were normalized to luciferase activity in cells exposed only to DMSO and are expressed as mean \pm SEM, $n=4,{ }^{*} p<0.05$. 


\section{Effect of Aroclor 1260 on the human farnesoid-X-receptor}

The ability of Aroclor 1260 to activate FXR, a key regulator of bile acid and energy metabolism was evaluated. HepG2 cells, co-transfected with pSG5-hFXR and pGL3-IR1-Luc were exposed to various concentrations of Aroclor 1260 for 24 h. GW4064 (0.5 $\mu \mathrm{M})$, a synthetic FXR agonist, was sed as a positive control. GW4064 activated FXR whereas Aroclor 1260 did not induce a response at any of the concentrations tested. In studies to test antagonism, all concentrations tested were without effect on the activation of human FXR (Fig. 4.7 A\&B). Thus, this PCB mixture is neither an agonist nor antagonist for the human FXR. 
A

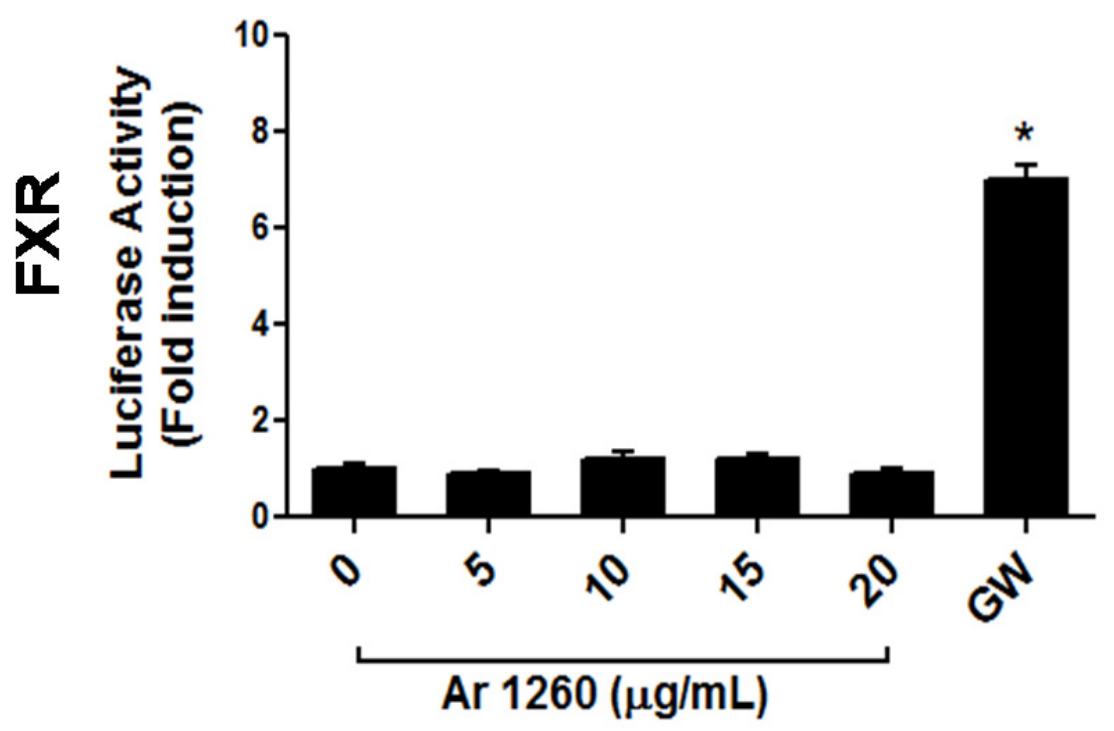

B

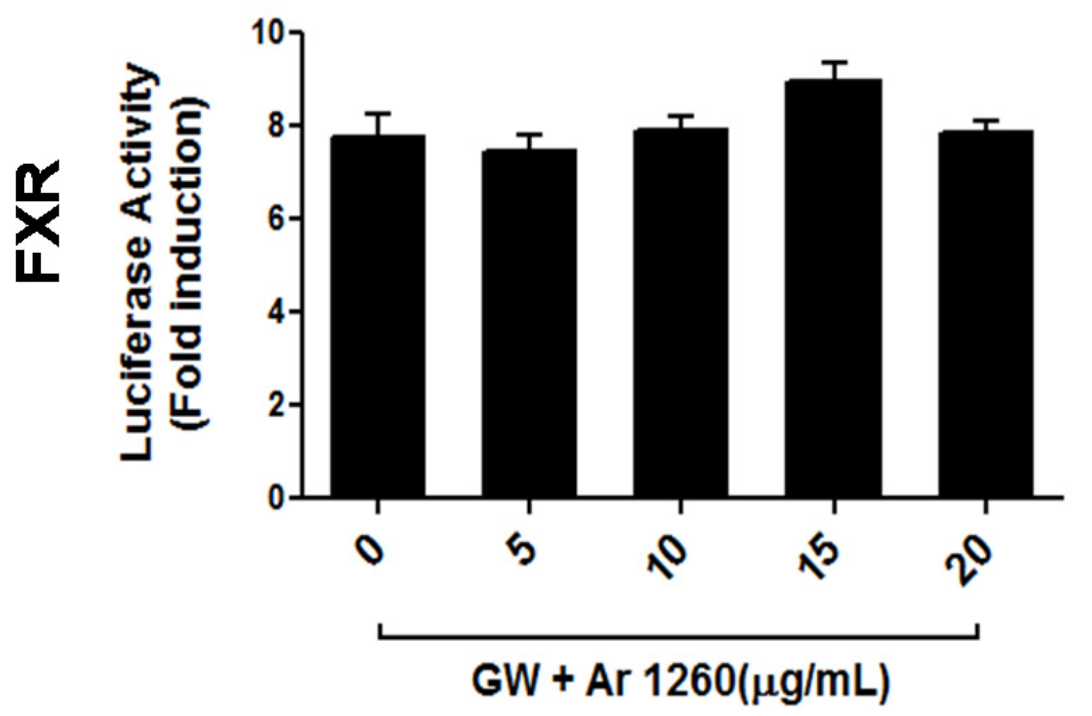


Figure 4.7. Aroclor 1260 activation of the human FXR.

HepG2 cells were transiently transfected with the expression plasmid pSG5-hFXR and reporter plasmid pGL3-IR1-Luc. GW4064 (0.5 $\mu \mathrm{M}, \mathrm{GW})$ was used as a positive control. (A) Cells were exposed to Aroclor 1260 at 0, 5, 10, 15 and $20 \mu \mathrm{g} / \mathrm{mL}$ and luciferase induction was normalized and compared to DMSOexposed cells ( $0 \mu \mathrm{g} / \mathrm{mL}$ Aroclor 1260). (B) Cells were exposed to $0.5 \mu \mathrm{M} \mathrm{GW}$ or GW plus Aroclor 1260 at $0,5,10,15$ and $20 \mu \mathrm{g} / \mathrm{mL}$. Luciferase activity in cells exposed to GW and Aroclor 1260 was compared to that of GW-exposed cells. Data were normalized to luciferase activity in cells exposed only to DMSO and are expressed as mean \pm SEM, $\mathrm{n}=4,{ }^{*} p<0.05$. 


\section{Effect of Aroclor 1260 on the human peroxisome-proliferator activated receptors}

PPARs are a group of nuclear receptors that mediate peroxisomal proliferation with subtype alpha $(\alpha)$ playing a distinct transcriptional role in lipid metabolism in the liver and the subtype gamma $(\mathrm{Y})$ in the adipose tissue. It was therefore important to evaluate the effect of Aroclor 1260 on PPARs $\alpha$ and $y$ activation. HepG2 cells were co-transfected with pCMV6-hPPARa and pGL3-

DR1-Luc. Nafenopin was used as a positive control. Aroclor 1260 did not activate PPARa at any of the concentrations (Fig. 4.8. A). In studies to test antagonism, Aroclor 1260 at the highest concentration $(20 \mu \mathrm{g} / \mathrm{mL})$ significantly antagonized PPAR $\alpha$ activation induced by nafenopin (27\%, reduction, $p=0.0222$, Fig. 4.8. B). Possible antagonism of PPARa by individual PCB congeners also was tested at $10 \mu \mathrm{M}$ concentration (Fig. 4.8. C). However, none of the selected congeners reduced the induction by nafenopin significantly, suggesting that a combination of the congeners is required to produce a marked effect or that other congeners present in this mixture may contribute to this effect.

For PPARY activation, HepG2 cells were co-transfected with pCMV6hPPARy and pGL3-DR1-Luc. Pioglitazone was used as a positive control. Aroclor 1260 did not activate PPARy nor antagonize PPARy activation by pioglitazone at any of the concentrations tested (Fig. 4.9. A\&B). 


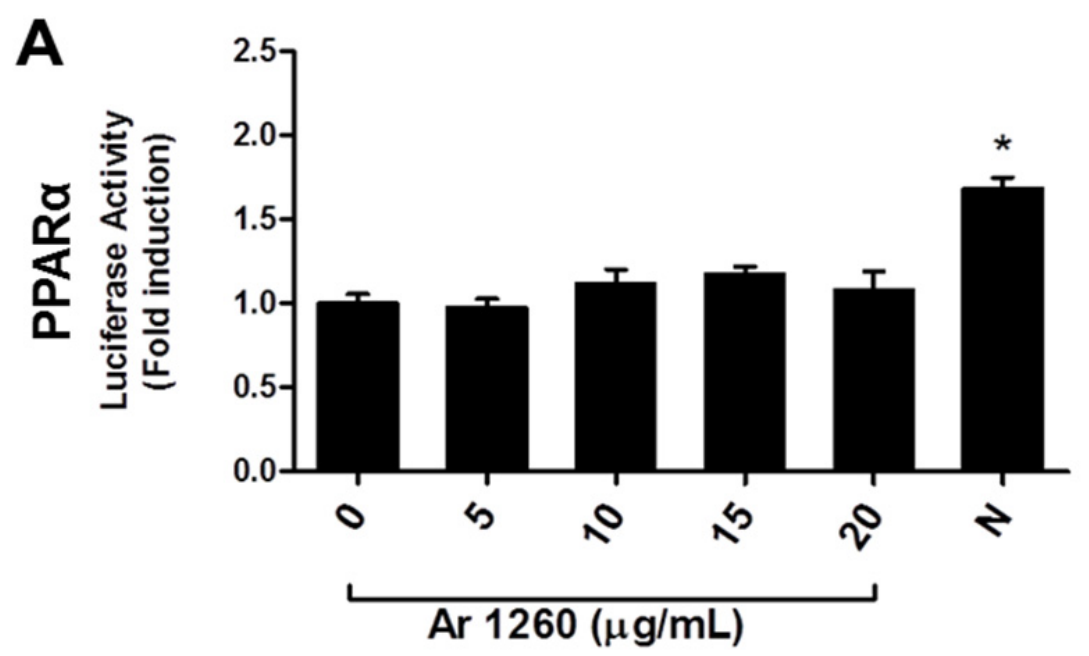

B
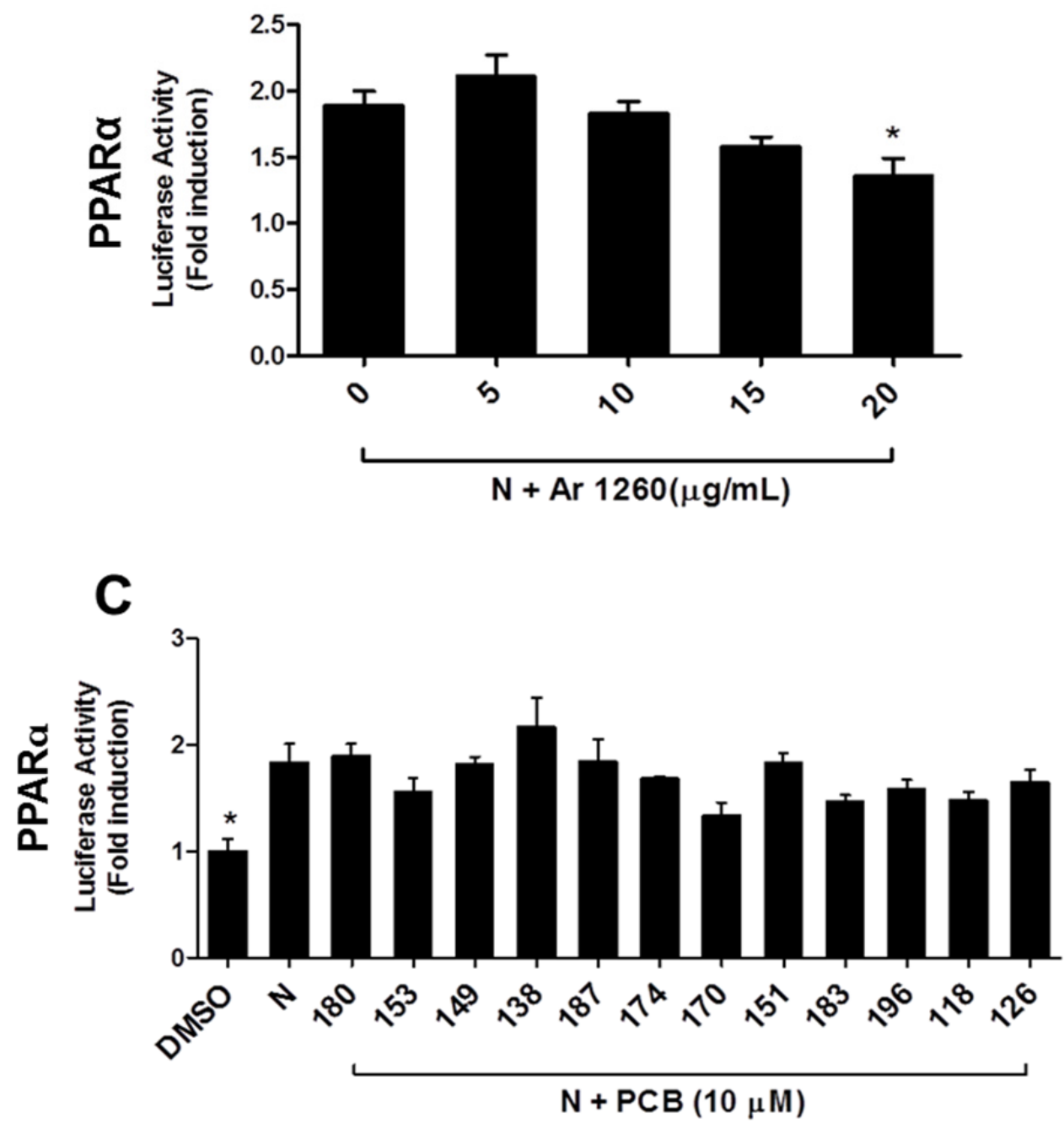


\section{Figure 4.8. Aroclor 1260 activation of the human PPARa.}

HepG2 cells were transiently transfected with the expression plasmid pCMV6-hPPARa and reporter plasmid pGL3-DR1-Luc. Nafenopin (50 $\mu \mathrm{M}, \mathrm{N})$ was used as a positive control. (A) Cells were exposed to Aroclor 1260 at 0, 5, 10,15 and $20 \mu \mathrm{g} / \mathrm{mL}$ and luciferase induction was normalized and compared to DMSO-exposed cells (0 $\mu \mathrm{g} / \mathrm{mL}$ Aroclor 1260). (B) Cells were exposed to $50 \mu \mathrm{M} \mathrm{N}$ or $\mathrm{N}$ plus Aroclor 1260 at $0,5,10,15$ and $20 \mu \mathrm{g} / \mathrm{mL}$. The luciferase induction was normalized to that of cells exposed only to DMSO solvent carrier (as in A, not shown). (C) Cells were exposed to selected PCB congeners $(10 \mu \mathrm{M})$ present in Aroclor 1260 and the induction was compared to DMSO-exposed cells. Luciferase activity in cells exposed to $\mathrm{N}$ and Aroclor 1260 was compared to that of $\mathrm{N}$-exposed cells. Data were normalized to luciferase activity in cells exposed only to DMSO and are expressed as mean \pm SEM, $n=4,{ }^{*} p<0.05$. Figure adapted from Wahlang et al., Toxicol Sci, 140 (2), 2014. 

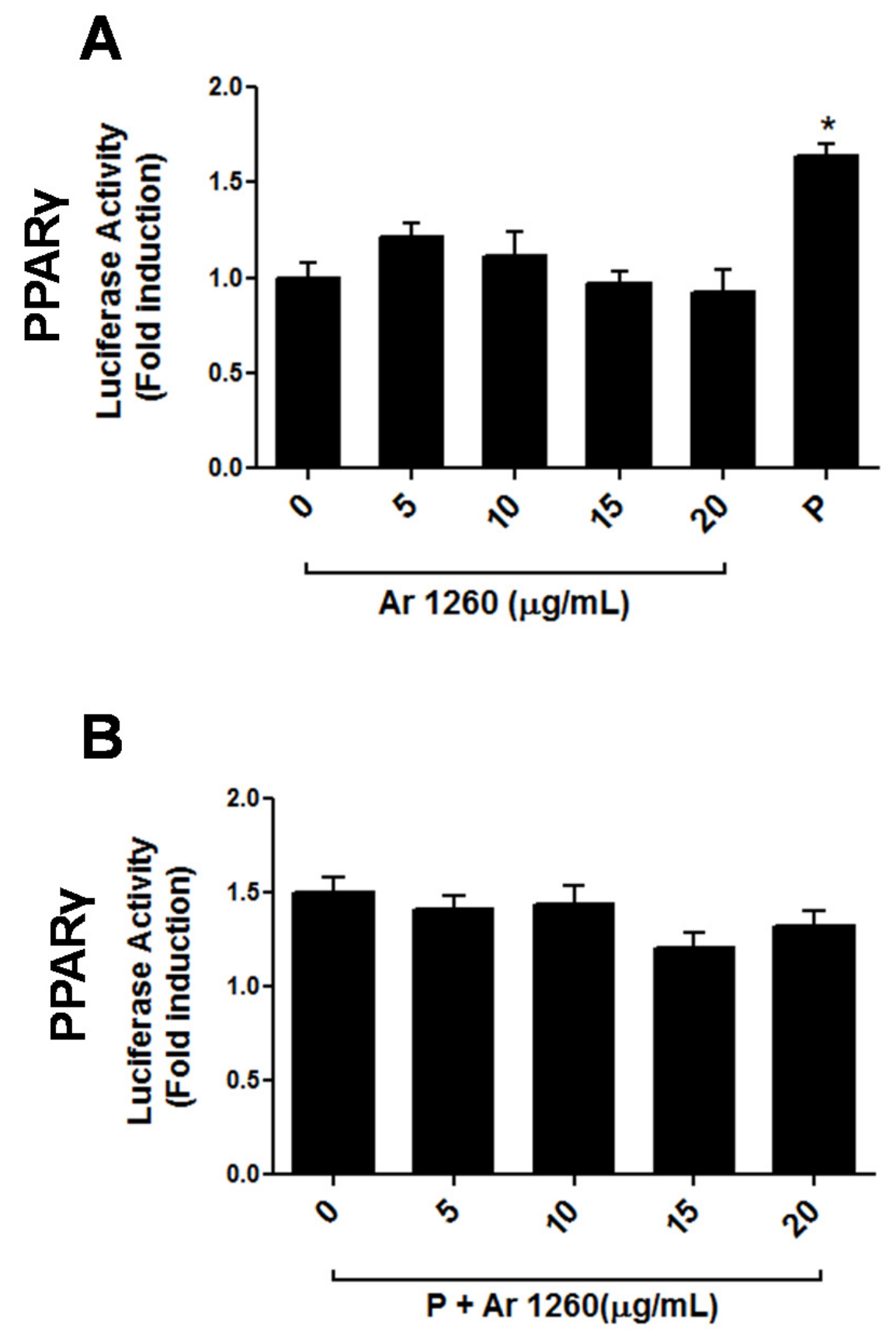
Figure 4.9. Aroclor 1260 activation of the human PPARY.

HepG2 cells were transiently transfected with the expression plasmid pCMV6-h PPARy and reporter plasmid pGL3-DR1-Luc. Pioglitazone $(10 \mu \mathrm{M}, \mathrm{P})$ was used as a positive control. (A) Cells were exposed to Aroclor 1260 at 0,5 , 10,15 and $20 \mu \mathrm{g} / \mathrm{mL}$ and luciferase induction was normalized and compared to DMSO-exposed cells (0 $\mu \mathrm{g} / \mathrm{mL}$ Aroclor 1260). (B) Cells were exposed to $10 \mu \mathrm{M} \mathrm{P}$ or $\mathrm{P}$ plus Aroclor 1260 at $0,5,10,15$ and $20 \mu \mathrm{g} / \mathrm{mL}$. Luciferase activity in cells exposed to P and Aroclor 1260 was compared to that of P-exposed cells. Data were normalized to luciferase activity in cells exposed only to DMSO and are expressed as mean \pm SEM, $n=4,{ }^{*} p<0.05$. 


\section{Aroclor 1260 exposure and gene expression in human hepatocytes}

To confirm our findings, we tested the effects of Aroclor 1260 exposure on target gene expression profiles in human hepatocytes. Primary hepatocytes were exposed to Aroclor 1260 at 5, 10, 15 and $20 \mu \mathrm{g} / \mathrm{mL}$ and gene expression was measured by RT-PCR. The mRNA expression of target genes namely CYP1A1, CD36, CYP3A4, CYP2B6, CPT1A, FAS and SHP were significantly induced by the respective receptor agonists in primary human hepatocytes (Fig. 4.10. A).

Expression levels of CYP1A1, an AhR target gene were significantly increased when hepatocytes were exposed to Aroclor $1260(15 \mu \mathrm{g} / \mathrm{mL}, p$ $=0.0018$, and $20 \mu \mathrm{g} / \mathrm{mL}, p=0.0066$, vs. unexposed cells) (Fig. 4.10. B). Contrary to CYP1A1, CD36, another AhR target gene was not induced by Aroclor 1260 exposure, suggesting that CD36 is not as inducible as CYP1A1 (Fig. 4.10. C). CD36 expression, however, is regulated by other transcription factors in addition to AhR (94). Furthermore, CYP3A4 (PXR target gene) mRNA expression was upregulated by Aroclor 1260 exposure at $15 \mu \mathrm{g} / \mathrm{mL}(p=0.0083)$ and $20 \mu \mathrm{g} / \mathrm{mL}(p$ $=0.0303)($ Fig. 4.10. D). Irrespective of the CAR2 antagonism observed in transfection assays, Aroclor 1260 induced CYP2B6 (CAR target gene) at 15 $\mu \mathrm{g} / \mathrm{mL}(p=0.0114)$ and $20 \mu \mathrm{g} / \mathrm{mL}(p=0.0036)$ (Fig. 4.10. E). Expression levels of other genes including FAS (LXR target gene), SHP (FXR target gene) and CPT1A (PPARa target gene) were not affected by Aroclor 1260 exposure (Fig. 4.10. F, G\&H respectively), thus confirming our results obtained from the HepG2 and COS-1 series of experiments. 
A B

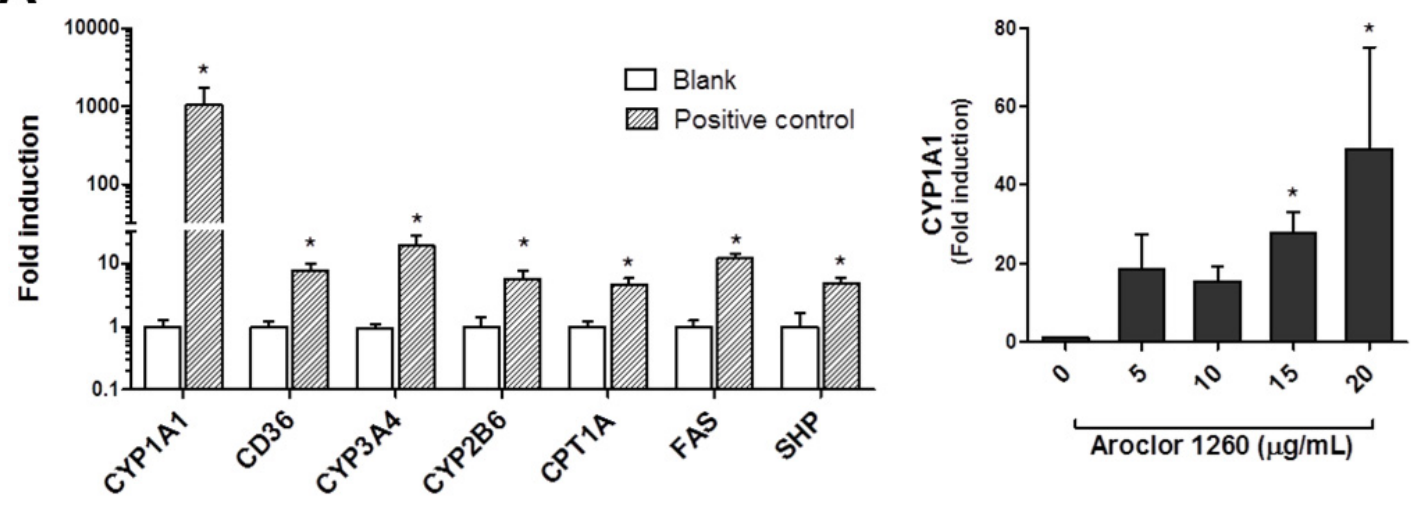

C

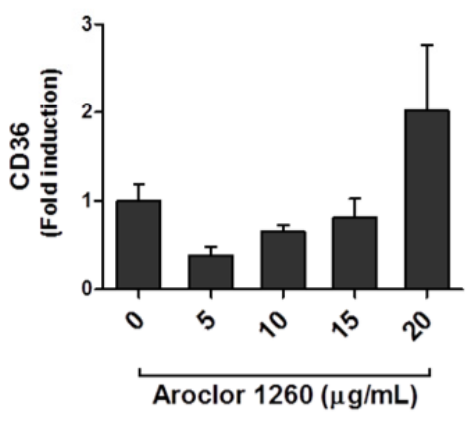

F

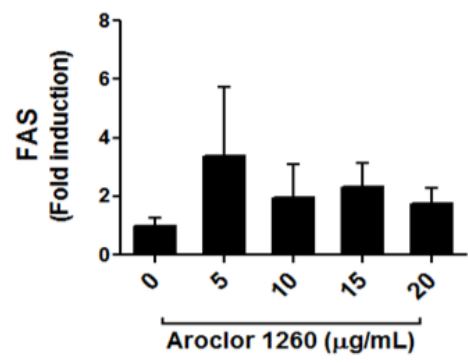

D

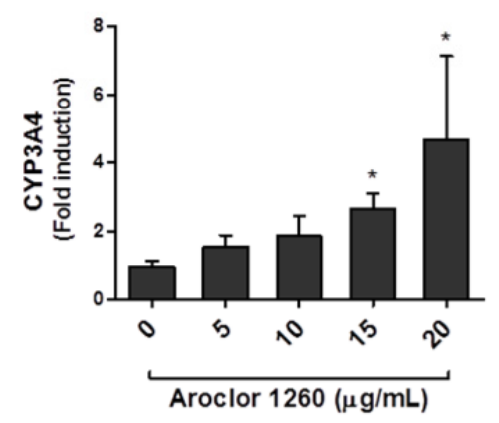

G

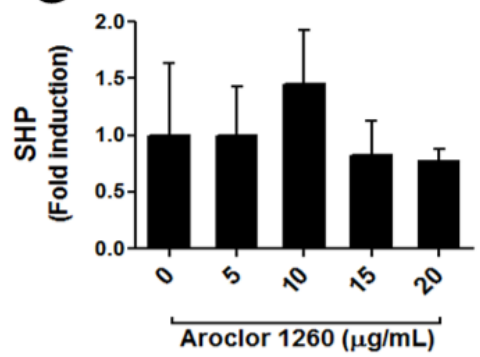

E
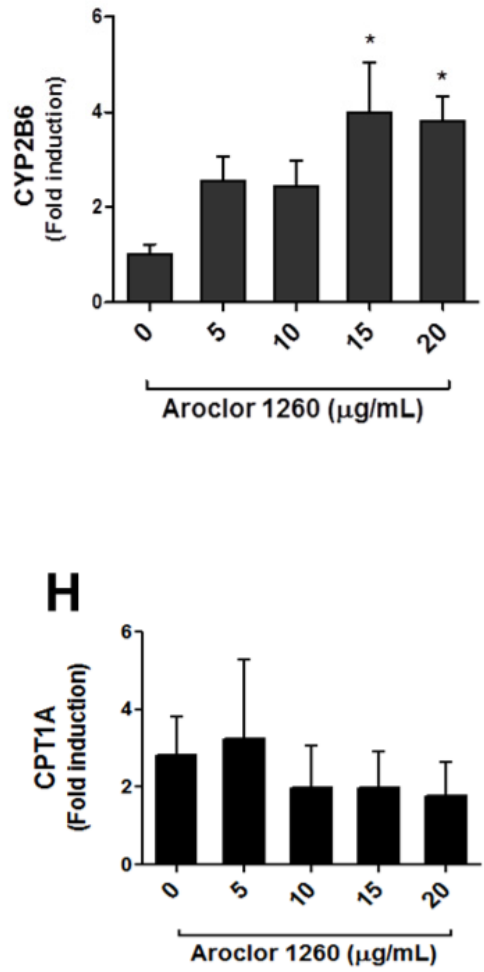
Figure 4.10. Effects of Aroclor 1260 on target genes in primary hepatocytes.

Primary human hepatocytes were exposed either to receptor ligands or different concentrations of Aroclor 1260. After a 24 hour incubation, RNA was isolated and RT-PCR was performed. (A) The mRNA levels of selected receptor target genes were upregulated by receptor ligands (positive control) namely BA $(10 \mu \mathrm{M})$ for CYP1A1 and CD36, RIF $(10 \mu \mathrm{M})$ for CYP3A4, CITCO $(10 \mu \mathrm{M})$ for CYP2B6, T0901317 (100 nM) for FAS, nafenopin (50 $\mu \mathrm{M})$ for CPT1A and GW4065 (0.5 $\mu \mathrm{M})$ for SHP. Relative mRNA levels of target genes were measured in Aroclor 1260-exposed cells namely (B) CYP1A1, (C) CD36, (D) CYP3A4, (E) CYP2B6, (F) FAS, (G) SHP and (H) CPT1A. Figure adapted from Wahlang et al., Toxicol Sci, 140 (2), 2014. 


\section{DISCUSSION}

Our laboratory recently identified suspected NAFLD and toxicantassociated steatohepatitis in NHANES participants with low-level environmental exposures to POPs, including 20 PCBs (18). Epidemiologic studies have shown a positive association between adipose tissue concentrations of PCBs and type 2 diabetes $(42,144)$. High serum PCB levels were also associated with elevated serum triglycerides and cholesterol which are major risk factors for cardiovascular diseases (145). Exposure to PCB mixtures has also been associated with elevated liver enzymes in plasma and hepatomegaly (53). Clearly, chronic exposures to these chlorinated compounds appear to disrupt both lipid and glucose homeostasis and consequently lead to diabetes and associated metabolic disorders such as NAFLD. Therefore, identifying mechanisms by which PCB exposure can lead to such deleterious effects is relevant to public health.

Environmental exposure to POPs such as PCBs is a major health concern, although subtly different from occupational exposure. The health effects from occupational exposures are more related to the smaller more metabolizable congeners and the subsequent formation of reactive potentially genotoxic metabolites. In contrast, the effects produced by the non-metabolizable PCBs in the body are expected to be chronic and life-long since they are not eliminated from the system. Aroclor 1260 production represented approximately $10.6 \%$ of total PCB production in the US between 1958-1977 which is modest compared to Aroclor 1242 (51.7\%) (146). Nonetheless, Aroclor 1260 was selected for this 
study because of the resemblance of congener composition pattern to that of human fat (36).

Lipid-adjusted serum PCB levels were measured in NHANES participants and the PCB exposed Anniston cohort and reported to range from 75-170 $\mathrm{ng} / \mathrm{g}$ $(0.1-0.5 \mu \mathrm{M})(18,46)$. Additionally, National Toxicology Program (NTP) studies reported the following PCB levels in a 2-year gavage study in rats: lipid-adjusted serum-4,650 ng/g; liver-64,593 ng/g; and adipose-2,495,994 ng/g (23). In these studies, PCB liver levels were at least 10-fold higher and PCB adipose levels were at least 200-fold higher than lipid-adjusted serum levels irrespective of the dose administered. In our experiments, we used Aroclor 1260 at concentrations ranging from $5-20 \mu \mathrm{g} / \mathrm{mL}(\sim 10-50 \mu \mathrm{M})$ and selected congeners at $10 \mu \mathrm{M}$. These concentrations should approximate the range seen in bio-accumulated organs/tissues such as the liver and adipose tissue.

Almost all PCB animal studies have been performed in rodents, where chronic toxicity is generally regarded as a function of the PCBs' ability to interact with $\mathrm{AhR}$ and nuclear receptors. There are clear differences in the ligand affinity of these receptors, in rodents and humans, toward model ligands and xenobiotic compounds, including PCBs. In order to understand more clearly the involvement of PCBs in human NAFLD as demonstrated in human epidemiological studies such as NHANES, it is important to understand specific interactions between PCBs and human receptors. The findings from the current study demonstrate that Aroclor 1260 activates PXR (Fig. 4.3) and to a lesser extent AhR (Fig. 4.2), suggesting that congener composition is critical in determining a mixture's 
mode(s) of action. The effect of Aroclor 1260 on the AhR is likely due to the presence of coplanar congeners such as PCB 126. However, because these coplanar compounds are only a small fraction of this mixture, a higher concentration of Aroclor 1260 would be required to observe the "dioxin-like" effects. PCB 138, a non-coplanar congener, may also be responsible for the Aroclor 1260 effect on the AhR. Parkinson et al demonstrated that some di-ortho substituted PCBs can induce hepatic microsomal benzo[a]pyrene hydroxylase, consistent with AhR activation. Thus, coplanarity does not necessarily define "dioxin like" PCBs from "non-dioxin like" ones (147).

The interaction between Aroclor 1260 and the AhR is further confirmed by the CYP1A1 induction at higher exposure levels in primary hepatocytes (Fig. 4.10). The CYP1A1 induction by Aroclor 1260 however was much less than the 1000-fold induction by the AhR ligand, BA. Furthermore, CD36, another AhR target gene was not induced by Aroclor 1260 vs. BA exposure in primary hepatocytes, further suggesting that a higher concentration of Aroclor 1260 may be required for "dioxin-like" effects. In contrast to AhR, Aroclor 1260 appears to be a relatively good human PXR agonist as observed through transient transfection assays and CYP3A4 induction in primary human hepatocytes. PXR is a transcription factor that plays a distinct role in drug metabolism and recent studies demonstrated its role in maintaining energy homeostasis in the body.

PXR activation is associated with decreased expression of genes involved in lipid catabolism namely carnitine palmitoyltransferase-1a and 3-hydroxy-3methylglutaryl-CoA-synthase and increased expression of the lipogenic gene 
stearoyl-CoA desaturase1 which could contribute to NAFLD (105). Exposure to PXR agonists also decreases blood glucose levels in fasting mice and this effect is related to forkhead box 01 (FoxO1) sequestration by PXR (74). A recent study by He et al. reported that ablation of this receptor in mice was protective against diet-induced and genetic obesity and improved insulin resistance (77). PXR activation clearly leads to disruption in the body's energy balance and this may be one of the mechanisms through which PCBs exert their effects on hepatic energy metabolism. Using transient transfection assays, Tabb et al. demonstrated that highly chlorinated PCBs including PCBs 184 and 197 inhibited human PXR (148). However, PCB 184 is not a component of Aroclor 1260 and PCB 197 accounts for only $0.07 \%$ of its total congener composition. This concentration is not likely sufficient to inhibit human PXR. In our study, the majority of PCBs in Aroclor 1260 appear to be PXR agonists.

As its name implies, wild type CAR is a constitutively active receptor due to a truncation that prevents internalization of the transactivation domain (AF2) and results in recruitment of co-activators. In vivo, CAR is regulated through its sequestration in the cytosol by chaperone proteins. Upon ligand binding, the receptor translocates to the nucleus and binds to its respective gene response elements. The cytosolic localization process is absent in immortalized cell lines. Consequently, performing transactivation studies in cell lines using in vitro reporter-based systems can be challenging due to the absence of ligand activation effect normally exhibited by CAR. However, in humans, the CAR transcript is spliced into several variants, in particular CAR2 and CAR3 that 
constitute approximately one third of the CAR mRNA pool and encode functional CAR proteins $(138,140)$. Interestingly, both CAR2 and CAR3 splice variants have additional amino acids within the receptor's ligand binding or heterodimerization domain that function to configure these variants as ligandactivated receptors, unlike the parent CAR. Aroclor 1260's interaction with human CAR is perhaps crucial, given the fact that non-coplanar PCBs, such as PCB 153, activate murine CAR target genes, including Cyp2b10. Of further interest, CAR has recently been identified as an anti-obesity nuclear receptor whose activation prevents obesity and improves type 2 diabetes through inhibition of lipogenesis and improved insulin sensitivity (72).

A recent study by Al-Salman et al. demonstrated that non-coplanar PCBs, namely PCBs 153, 180 and 194, are agonists for the human PXR and CAR at 10 $\mu \mathrm{M}$ (97). In that study, a receptor activation assay was performed in Huh7 cells using Checkmate mammalian two hybrid system where activated PXR/CAR bind to the DNA-binding domain of the NR Gal4 fusion plasmid. In the current investigation, we deployed a series of transactivation assays to evaluate the potential role of various PCB congeners to activate human CAR/PXR directly. In HepG2 cells, Aroclor 1260 activated CAR2 at $10 \mu \mathrm{g} / \mathrm{mL}$ but not at higher concentrations (Fig. 4.4). The mixed agonism/antagonism observed with CAR2 appeared concentration-dependent with antagonism seen at higher concentrations. In additional studies conducted with COS-1 cells, the results obtained with CAR3 were particularly intriguing, in that several PCB congeners were identified as human CAR activators (Fig. 4.5), in particular, PCBs 187, 183, 
149, 138, as well as Aroclor 1260 (ranked in decreasing order of potency). CAR3 is likely a highly sensitive and reasonable surrogate for CAR1, based on previous reports that include modeling of the receptors' ligand binding pockets $(140,149)$, whereas CAR2 appears to possess subtle alterations within its binding pocket that modify its ligand interaction profile compared with CAR1 (150).

In other experiments, Aroclor 1260 exposure induced the CAR target gene, CYP2B6 in human hepatocytes, indicating that this agent acts primarily as a CAR activator and not antagonist. This result is consistent with the activation profile exhibited by Aroclor 1260 on human CAR3 in the transactivation assay. However, it should be noted that ligand binding to CAR is not the sole mechanism by which CAR is activated. Recently, Mutoh et al. reported that phenobarbital, a well-known CAR agonist, indirectly activated CAR by inhibiting the epidermal growth factor receptor signaling which subsequently dephosphorylated CAR and mediated its translocation into the nucleus (151). We anticipate that some of the PCB congeners may not directly interact with CAR, but may act through the same mechanism as phenobarbital. Further studies will be required to test this possibility.

Previous studies showed that co-exposure to PCB 153 and high fat diet worsened NAFLD and obesity in male C57BI/6 mice through upregulation of fatty acid synthase, an LXRa target gene (48). However in the current study, Aroclor 1260 interacted with neither LXRa nor FXR. Although there was no direct effect by PCBs on either LXRa or FXR, we cannot rule out the possibility of an indirect interaction via cross-talk or alterations in nuclear receptor expression. However, 
nuclear receptor cross-talk is complex and not well established especially in relation to PCBs. Therefore more studies are required to elaborate on the possibility of cross-talk. Aroclor 1260 did not activate PPARa but it antagonized PPARa activation by nafenopin at higher concentrations (Fig. 4.8). However, none of the congeners tested at $10 \mu \mathrm{M}$ exhibited any antagonism. Robertson et al. reported the suppression of peroxisomal enzyme activities and CYP4A expression in male Sprague-Dawley rats treated with co-planar PCBs, including PCBs 77, 122, 126 and 169 (98). Considering the fact that Aroclor 1260 has a lesser percentage of coplanar PCBs than non-coplanar structural components, we may reconcile the possibility that a higher concentration of this PCB mixture may be required to antagonize PPAR $\alpha$ whereas PPARy remained unaffected.

In summary, Aroclor 1260 is an activator of human PXR and CAR 2/3 variants. It also appears to activate human $A h R$ and to antagonize human PPARa at higher concentrations. The current study is clinically relevant because human receptor activation by a PCB mixture that simulates PCB bioaccumulation in humans was examined at relevant concentrations. Furthermore, the effects of PCBs on human nuclear receptors such as LXR a and FXR and in human hepatocytes have never been assessed before. Additionally, this is the first study to evaluate PCB activation on the CAR variants. Because PCB exposures have been associated with obesity, NAFLD and metabolic syndrome and these disorders are intimately involved with nuclear receptor activation, the results clearly provide new insight into potential mode(s) of PCB action in human NAFLD. 
CHAPTER 5

OVERALL SUMMARY

\section{Overall goal and specific aims}

PCBs are persistent organic pollutants that dose-dependently increase the risk for liver injury as observed from epidemiologic studies. These polyhalogenated compounds persist in the ecosystem and the heavily chlorinated congeners bio-accumulate in living organisms. As stated previously, the commercial PCB mixture, Aroclor 1260 has a congener composition representative of bio-accumulated PCBs. Aroclor 1260 was therefore chosen for this dissertation project to simulate human PCB exposure patterns. Furthermore, PCBs are predicted to interact with most receptors previously implicated in xenobiotic/energy metabolism and NAFLD. These receptors include the AhR, PXR, CAR, PPARs, LXR $\alpha$ and FXR. To determine if PCB exposure play a causal role in the development of NAFLD and steatohepatitis, and to gain insight into the mechanisms involved, the in vivo studies were undertaken using Aroclor 1260 at doses that correlated to human PCB body burden. The overall objective of the dissertation project was to evaluate the role of PCB exposure, using Aroclor 1260, in fatty liver disease and to elucidate the potential PCB-receptor interactions that mediate fatty liver disease. To fulfill our objective, the following specific aims were proposed and carried out. 
1A. Develop a mouse model for PCB exposure that simulates human exposure paradigms.

1B. Evaluate the effects of the commercialized PCB mixture, Aroclor 1260, in obesity and NAFLD.

2. Determine the role of the nuclear receptors, CAR and PXR, in liver injury caused by Aroclor 1260 and high fat diet co-exposure.

3. Examine the interactions between Aroclor 1260/selected PCB congeners and human receptors in the liver

\section{Major findings of this dissertation}

The experiments described in Chapter 2, 3 and 4 addressed Specific Aim 1,2 and 3 respectively. The major findings from each Chapter are discussed in the following paragraphs.

\section{Evaluating the effects of Aroclor 1260 exposure in a diet-induced obesity mouse model}

Prior to initiating the animal studies, there were no documented studies on chronic HFD+PCB co-exposed animal models that simulate human exposure patterns. We therefore developed an animal model for studying the effects of PCB exposure at doses that were relevant to human exposure. Aroclor 1260 was administered to $\mathrm{C} 57 \mathrm{BI} / 6$ mice at 20 or $200 \mathrm{mg} / \mathrm{kg}$ via oral gavage in corn oil. The low dose $(20 \mathrm{mg} / \mathrm{kg})$ was designed to mimic the maximum human PCB exposures seen in the Anniston cohort and the high dose $(200 \mathrm{mg} / \mathrm{kg})$ was based on doses used in the NTP rodent carcinogenesis studies $(18,22)$. 
It was observed that relevant doses of Aroclor 1260 along with HFD coexposure caused toxicant-associated steatohepatitis in a mouse model of dietinduced obesity. In contrast to our previous study wherein a single congener (PCB 153) was used, this PCB mixture neither increased the body weight/visceral adiposity nor worsened insulin resistance/diet-induced obesity. On the contrary, Aroclor 1260 exposure was associated with decreased body fat in HFD-fed mice but had no effect on blood glucose/lipid levels. In fact, Aroclor 1260 at higher doses appeared to reduce diet-induced obesity by decreasing the $\%$ increase in bodyweight gain (Fig. 2.2). Paradoxically, Aroclor 1260+HFD coexposed mice demonstrated increased inflammatory foci at both doses while the degree of steatosis did not change. Serum cytokines (Fig. 2.5) and hepatic expression of IL-6 and TNFa (Fig. 2.9) were increased only at $20 \mathrm{mg} / \mathrm{kg}$, suggesting an inhibition of pro-inflammatory cytokine production at the $200 \mathrm{mg} / \mathrm{kg}$ exposure. Aroclor 1260 exposure induced hepatic expression of cytochrome P450s including Cyp3a11 (PXR target gene) and Cyp2b10 (CAR target gene) but Cyp2b10 inducibility was diminished with HFD-feeding (Fig. 2.10). Cyp1a2 (AhR target gene) was induced only at the higher dose $(200 \mathrm{mg} / \mathrm{kg})$. There was a significant difference between the low and high exposure doses in terms of hepatic/systemic inflammation, which could potentially be due to AhR activation. Aroclor 1260 activated CAR and PXR and to a lesser extent AhR, suggesting congener composition and exposure levels to be critical in determining a mixture's mode(s) of actions. 


\section{Evaluating the role of CAR and PXR in liver injury caused by Aroclor 1260 exposure in conjunction with HFD}

In Chapter 2, it was demonstrated that Aroclor 1260 at 20 and $200 \mathrm{mg} / \mathrm{kg}$ activated nuclear receptors CAR and PXR (152). In chapter 3, the role of CAR and PXR in Aroclor 1260+HFD-induced steatohepatitis was further evaluated. C57BI/6 (WT), PXR knockout and CAR knockout mice were exposed to Aroclor $1260(20 \mathrm{mg} / \mathrm{kg})$ and fed a high fat diet (42\% kCal fat) for 12 weeks. Consistent with our previous findings, Aroclor 1260 exposure resulted in the transition of diet-induced steatosis to steatohepatitis and this was independent of the mouse genotype (Fig. 3.5). Basal hepatic TNFa expression was increased in the knockout groups independent of exposure (Fig. 3.6), indicating an antiinflammatory role of these receptors in NASH. Moreover, the PXR knockout mice had increased \% body fat accompanied with decreased lean tissue mass and increased liver to body weight ratio, regardless of exposure (Fig. 3.1).

An intriguing finding from this study was that Aroclor 1260 exposure decreased serum insulin levels and HOMA-IR in all groups (Fig. 3.8). This finding correlated with findings from the Anniston Community Health Survey studies where serum insulin decreased with increasing PCB body burden (153). This was a paradoxical finding, because PCB exposure was associated with diabetes in this population and diabetes is normally associated with an increase in HOMA$I R$, hence increased insulin levels and insulin resistance. The results suggested that PCBs may have an effect on insulin secretion and pancreatic function. Interestingly, a recent chronic exposure study using Aroclor 1254 reported 
hyperinsulinemia in lean and diet-induced obese mice (49). However, the congener composition in Aroclor 1254 is strikingly different from that of Aroclor 1260 (146), with the former containing higher amounts of dioxin like PCBs. Therefore, congener composition as well as the dose used may play a role in the observed hyperinsulinemia.

The PXR knockout mice exposed to Aroclor 1260 showed a robust increase in hepatic gluconeogenic gene (PEPCK-1, G6Pase) expression (Fig. 3.9), suggesting that PXR may be a major repressor of the gluconeogenic transcription factor, FOXO1. In terms of lipogenic gene expression, Aroclor 1260 exposure decreased FAS and SCD1 mRNA levels in the WT group. However, the knockout groups had higher levels of basal FAS and SCD1 expression irrespective of Aroclor 1260 exposure (Fig. 3.11). This could be explained by the increase in basal mTOR1 activity in these groups as activated mTOR1 corresponds to an increase in lipogenesis through nuclear localization of the lipogenic transcription factor, SREBP1 (113). On the other hand, Aroclor 1260 exposure resulted in AMPKa activation which was consistent with an increase in hepatic expression of the glucose transporters (Fig. 3.13). AMPKa activation by Aroclor 1260 was manifested by the increase in RER in the knockout groups that were exposed to Aroclor 1260 (Fig. 3.3). Furthermore, both knockout groups demonstrated increased movement in the light cycle with Aroclor 1260 exposure, suggesting that this increase in physical activity may have driven AMPKa activation. However, the exact mechanism as to why Aroclor 1260 exposure caused hyperactivity in these animals remains to be investigated. 
In summary, CAR and PXR activation appeared to be protective with regard to inflammation, lipogenesis and gluconeogenesis. However, CAR and PXR activation was not sufficient to protect the animals from developing NASH. This was possibly due to mechanisms independent of CAR and PXR activation such as compromised insulin levels and no improvement in obesity inspite of the improved leptin to adiponectin ratio. Moreover, the NASH symptoms that were observed in these animals such as insulin resistance and steatosis were uncoupled as compared to typical NASH hallmarks. We conclude that although CAR /PXR activation played a protective role in NASH induced by PCB+HFD, there are other receptor-based mechanisms responsible for the development of NASH that need to be addressed in this PCB/HFD model.

\section{Human receptor activation by Aroclor 1260 and selected individual PCB congeners}

The studies in this Chapter evaluated Aroclor 1260 and selected congeners, as potential ligands for human receptors utilizing human hepatomaderived (HepG2) and primate-derived (COS-1) cell lines, and primary human hepatocytes. It is important to differentiate between rodent and human receptor activation because, although these receptors are highly conserved between species, some of these receptors such as the AhR vary in terms of ligand binding sites and target genes that they induce. The observed data suggested that Aroclor 1260 is a human AhR (Fig. 4.2), PXR (Fig. 4.3), a mixed agonist/antagonist for CAR2 (Fig. 4.4), a CAR3 agonist (Fig. 4.5) and an antagonist for human PPARa (Fig. 4.8). This study is clinically relevant because 
human receptor activation by a PCB mixture that simulates PCB bioaccumulation in humans was examined at relevant concentrations. Furthermore, the effects of PCBs on human nuclear receptors such as $L X R \alpha$ and FXR and in human hepatocytes have never been assessed before. Additionally, this is the first study to evaluate PCB activation on the human CAR variants. Because epidemiologic studies have reported associations between PCB exposure and NAFLD, and since these disorders are intimately involved with nuclear receptor activation, the results clearly provide new insight into potential mode(s) of PCB action in human NAFLD.

\section{Strengths of this dissertation}

There is much strength in this dissertation. First and foremost, an animal model that simulated human PCB exposure patterns was developed. Secondly, we have identified steatohepatitis caused by PCB exposure in a diet-induced obesity mouse model and validated that the effects of a PCB mixture (Aroclor 1260) vary from that of a single congener (PCB 153). The work has also provided insights in the underlying mechanism associated with PCB exposure such as dose- and diet-dependent activation of human and murine receptors including the AhR, CAR and PXR. Additionally, the results in this dissertation also answered key questions related to the role of CAR and PXR in hepatic energy metabolism and demonstrated the protective role of these receptors in PCB+HFD-induced liver injury.

Another major strength of this dissertation includes the identification of PCB-mediated NASH effects that are dissociated from typical NASH hallmarks 
such as the absence of insulin resistance. Furthermore, our observations of PCB-mediated NASH that resulted in a unique animal phenotype correlated with the observations seen in the PCB-exposed human population studies (153). Inspite of focusing on CAR and PXR, we have also distinguished other factors that play a role in PCB+HFD-induced liver injury such as the energy sensors, mTOR1 and AMPKa. The knockout studies demonstrated that chronic dysregulated receptor activation ensues in the PCB/HFD model that impact energy metabolism and liver injury.

\section{Limitations of this dissertation}

The primary limitation of this dissertation is that the hepatic and adipose concentrations of PCBs have not yet been quantified. This could potentially be done in the future using mass spectrophotometry. Quantifying the amount of PCBs in liver and fat will give us an idea of PCB compartmentalization in either a low fat diet or HFD setting. Another limitation in the animal studies is that the pancreatic tissues were not harvested for analysis. Therefore, we failed to look at the effects of Aroclor 1260 exposure on pancreatic function and subsequently, insulin secretion. An additional caveat in the experimental design of the in vivo studies is that the mice received Aroclor 1260 either at a single exposure (20 $\mathrm{mg} / \mathrm{kg}$ ) or four separate exposures $(50 \mathrm{mg} / \mathrm{kg})$ rather than the intermittent exposures that humans encounter from eating PCB- contaminated food. Furthermore, another drawback of this dissertation was using serum ALT/AST as a NASH biomarker based on low sensitivity, and evaluating other biomarkers is a possibility in future studies (50). In addition, for the knockout animal studies, a 
2x2 ANOVA using SigmaPlot was used for statistical analysis. However, a more rigorous approach would be using the SAS/SPSS Programs to perform contrast and multiple correction tests and this will be accomplished in the near future. Lastly, all our in vivo studies were performed using male mice; hence it is pertinent to note that the observed effects may vary with gender and species, especially since PCBs may also modulate the estrogen receptor.

\section{Future directions}

Although the studies described in the dissertation have answered numerous questions related to the effects of PCB exposure on NAFLD and obesity, the research studies have also raised a number of questions that need to be addressed further. Future research studies directed form this work are as follows:

\section{Evaluate the underlying mechanisms that resulted in decreased serum insulin levels with Aroclor 1260 exposure.}

The animal studies clearly pointed out that Aroclor 1260 exposure was associated with a decrease in serum insulin levels. However the exact mechanisms that led to decreased insulin levels were not evaluated in these studies. Insulin is synthesized within the $\beta$-cells of the Islets of Langerhans in the pancreas and its secretion is triggered by various stimuli including increased blood glucose levels (154). A 2-year study on a binary mixture of PCB 126 and PCB 153 by the NTP reported the development of pancreatic cancer in female SD rats (22). However, till date there are no studies reporting the effects of PCB exposure on pancreatic function, either in experimental animal models or in 
epidemiologic studies. Therefore, the first logical step toward understanding the mechanisms behind the PCB-mediated decreased serum insulin levels is to examine the effects of PCB exposure on pancreatic structure and function.

\section{Define the direct vs. indirect PCB activation of CAR.}

In Chapter 2 and 3, we demonstrated that Aroclor 1260 exposure activated CAR by inducing its target gene, Cyp 2b10. CAR activation can occur either by direct binding to the receptor itself or indirectly through epidermal growth factor receptor (EGFR) signaling. Phenobarbital, a prototypical CAR ligand activates CAR indirectly by inhibiting the EGFR signaling which subsequently dephosphorylated CAR and mediated its translocation into the nucleus (151). We anticipate that some of the PCB congeners may not directly interact with CAR, but may act through the same mechanism as phenobarbital. Further studies will be required to test this possibility. Interestingly, EGFR signaling promotes pancreatic $\beta$-cells expansion in response to a high nutrient state and in pregnancy $(155,156)$. It would be insightful to look at PCB-EGFR interactions since this may also answer questions related to the impact of PCB exposure on pancreatic function and insulin secretion.

\section{Investigate the effects of HFD feeding on CAR and PXR activation.}

Regardless of Aroclor 1260 exposure, it appeared that HFD consumption reduced the induction of CAR/PXR target genes as compared to CD consumption. HFD feeding reduced Cyp2b10 induction by approximately 235fold and 41 -fold $v s$. CD feeding at $20 \mathrm{mg} / \mathrm{kg}$ and $200 \mathrm{mg} / \mathrm{kg}$, respectively. These results appear consistent with previous studies $(100,101)$, and indicate that the 
ability of CAR to activate target genes is compromised when animals are fed with HFD. We speculate that dietary components may interfere with CAR/PXR activation and therefore, it would be interesting to look at the effects of other diets such as high fructose diet. Another possibility is that the diet-induced steatosis may alter PCB compartmentalization and therefore PCB concentrations in the liver may vary, leading to lowered CAR/PXR target gene induction.

\section{Study the neurological effects of PCB exposure pertaining to physical} activity.

Because metabolic chamber studies indicated that Aroclor 1260 exposure increased movement in the knockout mice, it is worthwhile to study the PCBmediated effects in the brain. Studies have shown different PCB congeners interacting with the ryanodine receptor $(157,158)$, as well as dopamine and GABA neurotransmission $(159,160)$, and this could potentially impact motor activity. Although looking at PCB-mediated neurological effects is beyond the scope of an environmental liver disease laboratory, we can however collaborate with other groups that study PCB-mediated effects on the central and peripheral nervous system.

\section{Elucidate the role of the AhR in PCB+HFD-induced liver disease.}

The role of the AhR in steatosis and NAFLD has been investigated with studies showing that AhR activation is consistent with upregulated CD36 and SCD1 (110, 161). Using an AhR knockout or an AhR/CAR/PXR triple knockout mouse model will provide insight into the role of the AhR in our PCB/HFD mouse model. Another approach in this context is to use a binary mixture of Aroclors 
such as Aroclor 1260 that has primarily non-dioxin like PCBs and Aroclor 1254 that has mostly dioxin like PCBs. This will result in a PCB mixture that has approximately 15 to 30 -fold more AhR activity than the one currently studied.

\section{Translate the animal findings to the PCB-exposed human population.}

The objective of this dissertation is to understand the role of PCB exposure in NAFLD, diabetes and obesity using animal models. We aim to translate our animal findings to the PCB-exposed human population so as to identify potential therapeutic targets that could be utilized to address the health disorders associated with this population. We will be using humanized AhR, CAR and PXR mice in our future studies to obtain data that is more relevant toward evaluating the PCB effects in humans.

\section{Conclusion}

Overall, Aroclor 1260 exposure resulted in the transition of steatosis to steatohepatitis in HFD-fed mice, thereby acting as a 'second hit' in diet-induced steatohepatitis. Aroclor 1260-induced NASH could involve activation of novel PCB receptors such as CAR and PXR or even non-receptor based modes of action. Both PXR and CAR are required to maximally attenuate Aroclor 1260induced changes in body composition, carbohydrate/lipid metabolism and inflammation in NASH. This is in stark contrast to hepatic steatosis induced by dioxin which is dependent on AhR activation (161). Also, CAR and PXR activation appeared to be protective in PCB-mediated toxicity but HFD consumption decreased the protective CAR/PXR activation, illustrating the importance of dietary co-exposures in PCB-related NASH (Fig. 5). Lastly, PCBs 
also interact directly with human receptors, implicating the potential role of these receptors as therapeutic targets for $\mathrm{NASH}$ and environmental liver disease. 


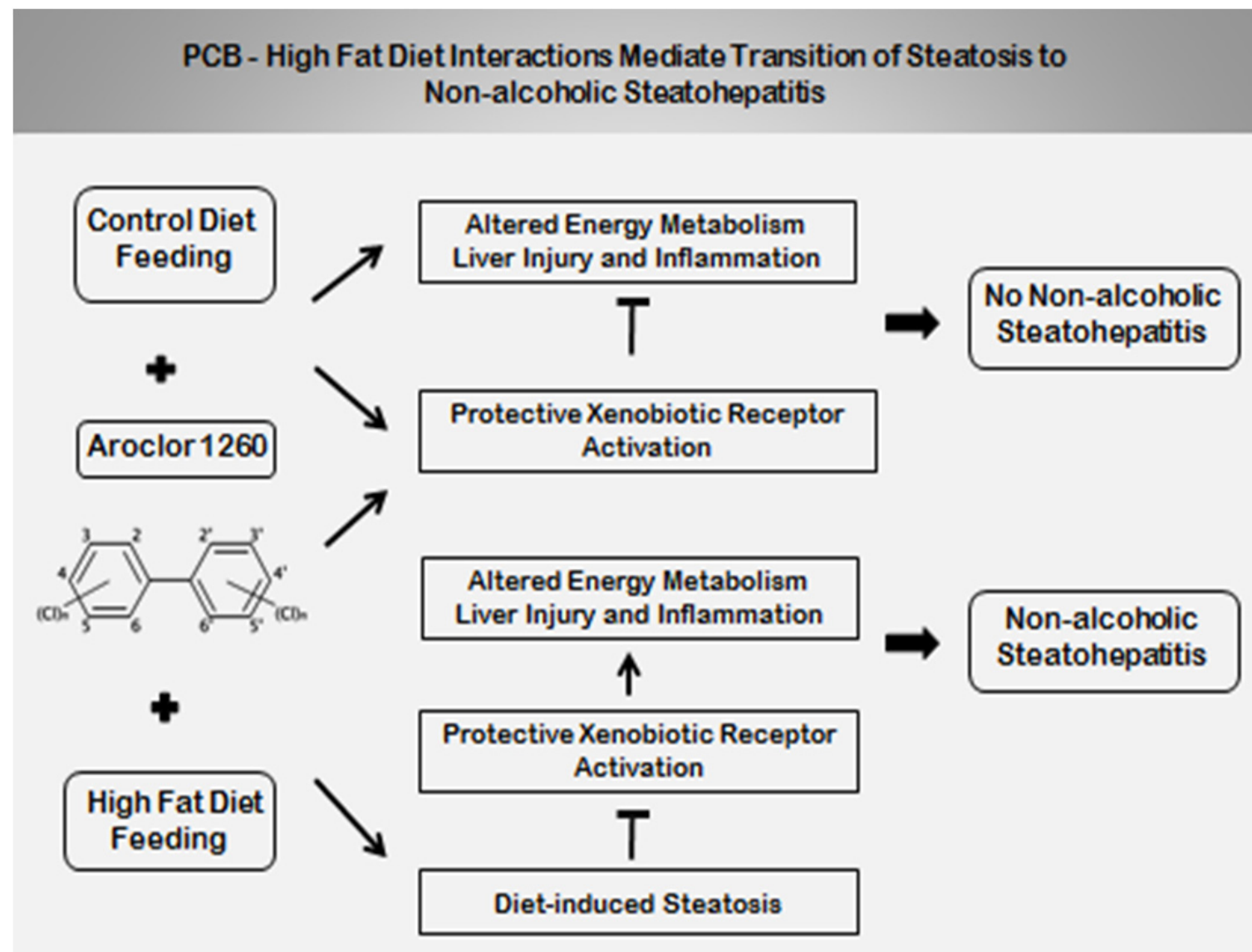

Figure 5.1. Environmentally-relevant PCB-diet interactions mediate the transition of steatosis to steatohepatitis. 


\section{REFERENCES}

1. Robertson LW, Hansen LG. PCBs: Recent Advances in Environmental Toxicology and Health Effects: University Press of Kentucky, 2001.

2. Giesy JP, Kannan K. Dioxin-like and non-dioxin-like toxic effects of polychlorinated biphenyls (PCBs): implications for risk assessment. Crit Rev Toxicol 1998;28:511-569.

3. Breivik K, Sweetman A, Pacyna JM, Jones KC. Towards a global historical emission inventory for selected PCB congeners--a mass balance approach. 1. Global production and consumption. Sci Total Environ 2002;290:181-198.

4. Porta M, Zumeta E. Implementing the Stockholm Treaty on Persistent Organic Pollutants. Occup Environ Med 2002;59:651-652.

5. Borja J, Taleon DM, Auresenia J, Gallardo S. Polychlorinated biphenyls and their biodegradation. Process Biochemistry 2005;40:1999-2013.

6. Rudel RA, Seryak LM, Brody JG. PCB-containing wood floor finish is a likely source of elevated PCBs in residents' blood, household air and dust: a case study of exposure. Environ Health 2008;7:2.

7. Perez-Maldonado IN, Salazar RC, llizaliturri-Hernandez CA, EspinosaReyes G, Perez-Vazquez FJ, Fernandez-Macias JC. Assessment of the polychlorinated biphenyls (PCBs) levels in soil samples near an electric 
capacitor manufacturing industry in Morelos, Mexico. J Environ Sci Health A Tox Hazard Subst Environ Eng 2014;49:1244-1250.

8. Marek RF, Martinez A, Hornbuckle KC. Discovery of hydroxylated polychlorinated biphenyls (OH-PCBs) in sediment from a lake Michigan waterway and original commercial aroclors. Environ Sci Technol 2013;47:8204-8210.

9. Tomza-Marciniak A, Marciniak A, Pilarczyk B, Drozd R, Ligocki M, Prokulewicz A. Wild boar (Sus scrofa) as a bioindicator of organochlorine compound contamination in terrestrial ecosystems of West Pomerania Province, NW Poland. Environ Monit Assess 2014;186:229-238.

10. Bodin N, Tapie N, Le Menach K, Chassot E, Elie P, Rochard E, Budzinski H. PCB contamination in fish community from the Gironde Estuary (France): blast from the past. Chemosphere 2014;98:66-72.

11. Covaci A, Voorspoels S, Roosens L, Jacobs W, Blust R, Neels H. Polybrominated diphenyl ethers (PBDEs) and polychlorinated biphenyls (PCBs) in human liver and adipose tissue samples from Belgium. Chemosphere 2008;73:170-175.

12. Polder A, Skaare JU, Skjerve E, Loken KB, Eggesbo M. Levels of chlorinated pesticides and polychlorinated biphenyls in Norwegian breast milk (2002-2006), and factors that may predict the level of contamination. Sci Total Environ 2009;407:4584-4590. 
13. Cimenci O, Vandevijvere S, Goscinny S, Van Den Bergh MA, Hanot V, Vinkx C, Bolle F, et al. Dietary exposure of the Belgian adult population to non-dioxin-like PCBs. Food Chem Toxicol 2013;59:670-679.

14. Malisch R, Kotz A. Dioxins and PCBs in feed and food - Review from European perspective. Sci Total Environ 2014;491-492C:2-10.

15. Tue NM, Takahashi S, Suzuki G, Isobe T, Viet PH, Kobara Y, Seike N, et al. Contamination of indoor dust and air by polychlorinated biphenyls and brominated flame retardants and relevance of non-dietary exposure in Vietnamese informal e-waste recycling sites. Environ Int 2013;51:160-167.

16. Hu X, Adamcakova-Dodd A, Lehmler HJ, Hu D, Hornbuckle K, Thorne PS. Subchronic inhalation exposure study of an airborne polychlorinated biphenyl mixture resembling the Chicago ambient air congener profile. Environ Sci Technol 2012;46:9653-9662.

17. Schecter A, Colacino J, Haffner D, Patel K, Opel M, Papke O, Birnbaum L. Perfluorinated compounds, polychlorinated biphenyls, and organochlorine pesticide contamination in composite food samples from Dallas, Texas, USA. Environ Health Perspect 2010;118:796-802.

18. Cave M, Appana S, Patel M, Falkner KC, McClain CJ, Brock G. Polychlorinated biphenyls, lead, and mercury are associated with liver disease in American adults: NHANES 2003-2004. Environ Health Perspect 2010;118:1735-1742.

19. ATSDR. (Agency for Toxic Substances and Disease Registry). Toxicological profile for polychlorinated biphenyls (update). U.S. 
Department of Health and Human Services, Public Health Service, Atlanta, GA, 2000.

20. Safe S, Bandiera S, Sawyer T, Robertson L, Safe L, Parkinson A, Thomas PE, et al. PCBs: structure-function relationships and mechanism of action. Environ Health Perspect 1985;60:47-56.

21. Safe S. Toxicology, structure-function relationship, and human and environmental health impacts of polychlorinated biphenyls: progress and problems. Environ Health Perspect 1993;100:259-268.

22. NTP. (National Toxicology Program). Toxicology and carcinogenesis studies of a binary mixture of $3,3^{\prime}, 4,4^{\prime}, 5$-pentachlorobiphenyl (PCB 126) (Cas No. 57465-28-8) and 2,2',4,4',5,5'-hexachlorobiphenyl (PCB 153) (CAS No. 35065-27-1) in female Harlan Sprague-Dawley rats (gavage studies). Natl Toxicol Program Tech Rep Ser 2006:1-258.

23. NTP. (National Toxicology Program). NTP technical report on the toxicology and carcinogenesis studies of $2,2^{\prime}, 4,4^{\prime}, 5,5^{\prime}$-hexachlorobiphenyl (PCB 153) (CAS No. 35065-27-1) in female Harlan Sprague-Dawley rats (Gavage studies). Natl Toxicol Program Tech Rep Ser 2006:4-168.

24. Kopec AK, Burgoon LD, Ibrahim-Aibo D, Mets BD, Tashiro C, Potter D, Sharratt B, et al. PCB153-elicited hepatic responses in the immature, ovariectomized C57BL/6 mice: comparative toxicogenomic effects of dioxin and non-dioxin-like ligands. Toxicol Appl Pharmacol 2010;243:359371. 
25. Luthe G, Jacobus JA, Robertson LW. Receptor interactions by polybrominated diphenyl ethers versus polychlorinated biphenyls: a theoretical Structure-activity assessment. Environ Toxicol Pharmacol 2008;25:202-210.

26. NTP. (National Toxicology Program). Toxicology and carcinogenesis studies of a binary mixture of $3,3^{\prime}, 4,4^{\prime}, 5$-pentachlorobiphenyl (PCB 126) (Cas No. 57465-28-8) and 2,3',4,4',5-pentachlorobiphenyl (PCB 118) (Cas No. 31508-00-6) in female Harlan Sprague-Dawley rats (gavage studies). Natl Toxicol Program Tech Rep Ser 2006:1-218.

27. Dean CE, Jr., Benjamin SA, Chubb LS, Tessari JD, Keefe TJ. Nonadditive hepatic tumor promoting effects by a mixture of two structurally different polychlorinated biphenyls in female rat livers. Toxicol Sci 2002;66:54-61.

28. Dere E, Lee AW, Burgoon LD, Zacharewski TR. Differences in TCDDelicited gene expression profiles in human HepG2, mouse Hepa1c1c7 and rat H4IIE hepatoma cells. BMC Genomics 2011;12:193.

29. Brown JF. Determination of PCB Metabolic, Excretion, and Accumulation Rates for Use as Indicators of Biological Response and Relative Risk. Environ Sci Technol 1994;28:2295-2305.

30. Aylward LL, Collins JJ, Bodner KM, Wilken M, Bodnar CM. "Intrinsic" elimination rate and dietary intake estimates for selected indicator PCBs: Toxicokinetic modeling using serial sampling data in US subjects, 20052010. Chemosphere 2014;110:48-52. 
31. Hennig B, Ettinger AS, Jandacek RJ, Koo S, McClain C, Seifried H, Silverstone A, et al. Using nutrition for intervention and prevention against environmental chemical toxicity and associated diseases. Environ Health Perspect 2007;115:493-495.

32. Cave M, Deaciuc I, Mendez C, Song Z, Joshi-Barve S, Barve S, McClain C. Nonalcoholic fatty liver disease: predisposing factors and the role of nutrition. J Nutr Biochem 2007;18:184-195.

33. Pavuk M, Olson JR, Sjodin A, Wolff P, Turner WE, Shelton C, Dutton ND, et al. Serum concentrations of polychlorinated biphenyls (PCBs) in participants of the Anniston Community Health Survey. Sci Total Environ 2014;473-474:286-297.

34. Aminov Z, Haase RF, Pavuk M, Carpenter DO. Analysis of the effects of exposure to polychlorinated biphenyls and chlorinated pesticides on serum lipid levels in residents of Anniston, Alabama. Environ Health 2013;12:108.

35. Beyer A, Biziuk M. Environmental fate and global distribution of polychlorinated biphenyls. Rev Environ Contam Toxicol 2009;201:137158.

36. McFarland VA, Clarke JU. Environmental occurrence, abundance, and potential toxicity of polychlorinated biphenyl congeners: considerations for a congener-specific analysis. Environ Health Perspect 1989;81:225-239.

37. Bertazzi PA, Riboldi L, Pesatori A, Radice L, Zocchetti C. Cancer mortality of capacitor manufacturing workers. Am J Ind Med 1987;11:165-176. 
38. Cogliano VJ. Assessing the cancer risk from environmental PCBs. Environ Health Perspect 1998;106:317-323.

39. Winneke G, Bucholski A, Heinzow B, Kramer U, Schmidt E, Walkowiak J, Wiener JA, et al. Developmental neurotoxicity of polychlorinated biphenyls (PCBS): cognitive and psychomotor functions in 7-month old children. Toxicol Lett 1998;102-103:423-428.

40. Aoki Y. Polychlorinated biphenyls, polychlorinated dibenzo-p-dioxins, and polychlorinated dibenzofurans as endocrine disrupters--what we have learned from Yusho disease. Environ Res 2001;86:2-11.

41. Ruzzin J, Petersen R, Meugnier E, Madsen L, Lock EJ, Lillefosse H, Ma T, et al. Persistent organic pollutant exposure leads to insulin resistance syndrome. Environ Health Perspect 2010;118:465-471.

42. Silverstone AE, Rosenbaum PF, Weinstock RS, Bartell SM, Foushee HR, Shelton C, Pavuk M. Polychlorinated biphenyl (PCB) exposure and diabetes: results from the Anniston Community Health Survey. Environ Health Perspect 2012;120:727-732.

43. Tang-Peronard JL, Heitmann BL, Andersen HR, Steuerwald U, Grandjean P, Weihe P, Jensen TK. Association between prenatal polychlorinated biphenyl exposure and obesity development at ages 5 and $7 \mathrm{y}$ : a prospective cohort study of 656 children from the Faroe Islands. Am J Clin Nutr 2014;99:5-13.

44. Lee DH, Lee IK, Porta M, Steffes M, Jacobs DR, Jr. Relationship between serum concentrations of persistent organic pollutants and the prevalence 
of metabolic syndrome among non-diabetic adults: results from the National Health and Nutrition Examination Survey 1999-2002.

Diabetologia 2007;50:1841-1851.

45. Goncharov A, Haase RF, Santiago-Rivera A, Morse G, McCaffrey RJ, Rej R, Carpenter DO. High serum PCBs are associated with elevation of serum lipids and cardiovascular disease in a Native American population. Environ Res 2008;106:226-239.

46. Goncharov A, Pavuk M, Foushee HR, Carpenter DO. Blood pressure in relation to concentrations of PCB congeners and chlorinated pesticides. Environ Health Perspect 2011;119:319-325.

47. Yu ML, Guo YL, Hsu CC, Rogan WJ. Increased mortality from chronic liver disease and cirrhosis 13 years after the Taiwan "yucheng" ("oil disease") incident. Am J Ind Med 1997;31:172-175.

48. Wahlang B, Falkner KC, Gregory B, Ansert D, Young D, Conklin DJ, Bhatnagar A, et al. Polychlorinated biphenyl 153 is a diet-dependent obesogen that worsens nonalcoholic fatty liver disease in male C57BL6/J mice. J Nutr Biochem 2013;24:1587-1595.

49. Gray SL, Shaw AC, Gagne AX, Chan HM. Chronic exposure to PCBs (Aroclor 1254) exacerbates obesity-induced insulin resistance and hyperinsulinemia in mice. J Toxicol Environ Health A 2013;76:701-715.

50. Cave M, Falkner KC, Ray M, Joshi-Barve S, Brock G, Khan R, Bon Homme M, et al. Toxicant-associated steatohepatitis in vinyl chloride workers. Hepatology 2010;51:474-481. 
51. Wahlang B, Beier JI, Clair HB, Bellis-Jones HJ, Falkner KC, McClain CJ, Cave MC. Toxicant-associated steatohepatitis. Toxicol Pathol 2013;41:343-360.

52. Kim KS, Lee YM, Kim SG, Lee IK, Lee HJ, Kim JH, Kim J, et al. Associations of organochlorine pesticides and polychlorinated biphenyls in visceral vs. subcutaneous adipose tissue with type 2 diabetes and insulin resistance. Chemosphere 2014;94:151-157.

53. Maroni M, Colombi A, Arbosti G, Cantoni S, Foa V. Occupational exposure to polychlorinated biphenyls in electrical workers. II. Health effects. Br J Ind Med 1981;38:55-60.

54. Abdelmalek MF, Suzuki A, Guy C, Unalp-Arida A, Colvin R, Johnson RJ, Diehl AM. Increased fructose consumption is associated with fibrosis severity in patients with nonalcoholic fatty liver disease. Hepatology 2010;51:1961-1971.

55. Seki E, Brenner DA. Toll-like receptors and adaptor molecules in liver disease: update. Hepatology 2008;48:322-335.

56. Browning JD, Horton JD. Molecular mediators of hepatic steatosis and liver injury. J Clin Invest 2004;114:147-152.

57. Chiang DJ, Pritchard MT, Nagy LE. Obesity, diabetes mellitus, and liver fibrosis. Am J Physiol Gastrointest Liver Physiol 2011;300:G697-702.

58. Mangelsdorf DJ, Thummel C, Beato M, Herrlich P, Schutz G, Umesono K, Blumberg B, et al. The nuclear receptor superfamily: the second decade. Cell 1995;83:835-839. 
59. Novac N, Heinzel T. Nuclear receptors: overview and classification. Curr Drug Targets Inflamm Allergy 2004;3:335-346.

60. Konno Y, Negishi M, Kodama S. The roles of nuclear receptors CAR and PXR in hepatic energy metabolism. Drug Metab Pharmacokinet 2008;23:8-13.

61. Merrell MD, Cherrington NJ. Drug metabolism alterations in nonalcoholic fatty liver disease. Drug Metab Rev 2011;43:317-334.

62. Moya M, Gomez-Lechon MJ, Castell JV, Jover R. Enhanced steatosis by nuclear receptor ligands: a study in cultured human hepatocytes and hepatoma cells with a characterized nuclear receptor expression profile. Chem Biol Interact 2010;184:376-387.

63. Ko HP, Okino ST, Ma Q, Whitlock JP, Jr. Dioxin-induced CYP1A1 transcription in vivo: the aromatic hydrocarbon receptor mediates transactivation, enhancer-promoter communication, and changes in chromatin structure. Mol Cell Biol 1996;16:430-436.

64. Nebert DW, Roe AL, Dieter MZ, Solis WA, Yang Y, Dalton TP. Role of the aromatic hydrocarbon receptor and [Ah] gene battery in the oxidative stress response, cell cycle control, and apoptosis. Biochem Pharmacol 2000;59:65-85.

65. Gasiewicz TA, Singh KP, Casado FL. The aryl hydrocarbon receptor has an important role in the regulation of hematopoiesis: implications for benzene-induced hematopoietic toxicity. Chem Biol Interact 2010;184:246-251. 
66. Li Y, Innocentin S, Withers DR, Roberts NA, Gallagher AR, Grigorieva EF, Wilhelm C, et al. Exogenous stimuli maintain intraepithelial lymphocytes via aryl hydrocarbon receptor activation. Cell 2011;147:629-640.

67. Hennig B, Reiterer G, Toborek M, Matveev SV, Daugherty A, Smart E, Robertson LW. Dietary fat interacts with PCBs to induce changes in lipid metabolism in mice deficient in low-density lipoprotein receptor. Environ Health Perspect 2005;113:83-87.

68. Lee JH, Wada T, Febbraio M, He J, Matsubara T, Lee MJ, Gonzalez FJ, et al. A novel role for the dioxin receptor in fatty acid metabolism and hepatic steatosis. Gastroenterology 2010;139:653-663.

69. Kliewer SA, Goodwin B, Willson TM. The nuclear pregnane X receptor: a key regulator of xenobiotic metabolism. Endocr Rev 2002;23:687-702.

70. Wada T, Gao J, Xie W. PXR and CAR in energy metabolism. Trends Endocrinol Metab 2009;20:273-279.

71. Dong B, Saha PK, Huang W, Chen W, Abu-Elheiga LA, Wakil SJ, Stevens $\mathrm{RD}$, et al. Activation of nuclear receptor CAR ameliorates diabetes and fatty liver disease. Proc Natl Acad Sci U S A 2009;106:18831-18836.

72. Gao J, He J, Zhai Y, Wada T, Xie W. The constitutive androstane receptor is an anti-obesity nuclear receptor that improves insulin sensitivity. J Biol Chem 2009;284:25984-25992.

73. Roth A, Looser R, Kaufmann M, Blattler SM, Rencurel F, Huang W, Moore DD, et al. Regulatory cross-talk between drug metabolism and lipid 
homeostasis: constitutive androstane receptor and pregnane $\mathrm{X}$ receptor increase Insig-1 expression. Mol Pharmacol 2008;73:1282-1289.

74. Kodama S, Koike C, Negishi M, Yamamoto Y. Nuclear receptors CAR and PXR cross talk with FOXO1 to regulate genes that encode drugmetabolizing and gluconeogenic enzymes. Mol Cell Biol 2004;24:79317940.

75. Ma Y, Liu D. Activation of pregnane $\mathrm{X}$ receptor by pregnenolone 16 alphacarbonitrile prevents high-fat diet-induced obesity in AKR/J mice. PLoS One 2012;7:e38734.

76. Spruiell K, Richardson RM, Cullen JM, Awumey EM, Gonzalez FJ, Gyamfi MA. Role of pregnane $\mathrm{X}$ receptor in obesity and glucose homeostasis in male mice. J Biol Chem 2014;289:3244-3261.

77. He J, Gao J, Xu M, Ren S, Stefanovic-Racic M, O'Doherty RM, Xie W. PXR ablation alleviates diet-induced and genetic obesity and insulin resistance in mice. Diabetes 2013;62:1876-1887.

78. Khorasanizadeh S, Rastinejad F. Nuclear-receptor interactions on DNAresponse elements. Trends Biochem Sci 2001;26:384-390.

79. Williams CD, Stengel J, Asike MI, Torres DM, Shaw J, Contreras M, Landt $\mathrm{CL}$, et al. Prevalence of nonalcoholic fatty liver disease and nonalcoholic steatohepatitis among a largely middle-aged population utilizing ultrasound and liver biopsy: a prospective study. Gastroenterology 2011;140:124-131. 
80. Lazo M, Hernaez R, Bonekamp S, Kamel IR, Brancati FL, Guallar E, Clark JM. Non-alcoholic fatty liver disease and mortality among US adults: prospective cohort study. BMJ 2011;343:d6891.

81. Vernon G, Baranova A, Younossi ZM. Systematic review: the epidemiology and natural history of non-alcoholic fatty liver disease and non-alcoholic steatohepatitis in adults. Aliment Pharmacol Ther $2011 ; 34: 274-285$.

82. Flegal KM, Carroll MD, Ogden CL, Johnson CL. Prevalence and trends in obesity among US adults, 1999-2000. JAMA 2002;288:1723-1727.

83. Finkelstein EA, Trogdon JG, Cohen JW, Dietz W. Annual medical spending attributable to obesity: payer-and service-specific estimates. Health Aff (Millwood) 2009;28:w822-831.

84. Ogden CL, Carroll MD, Kit BK, Flegal KM. Prevalence of childhood and adult obesity in the United States, 2011-2012. JAMA 2014;311:806-814.

85. Jeppesen J, Hansen TW, Rasmussen S, Ibsen H, Torp-Pedersen C, Madsbad S. Insulin resistance, the metabolic syndrome, and risk of incident cardiovascular disease: a population-based study. J Am Coll Cardiol 2007;49:2112-2119.

86. O'Neill S, O'Driscoll L. Metabolic syndrome: a closer look at the growing epidemic and its associated pathologies. Obes Rev 2014.

87. Nashar K, Egan BM. Relationship between chronic kidney disease and metabolic syndrome: current perspectives. Diabetes Metab Syndr Obes 2014;7:421-435. 
88. Dowman JK, Tomlinson JW, Newsome PN. Pathogenesis of non-alcoholic fatty liver disease. QJM 2010;103:71-83.

89. Seth RK, Kumar A, Das S, Kadiiska MB, Michelotti G, Diehl AM, Chatterjee S. Environmental toxin-linked nonalcoholic steatohepatitis and hepatic metabolic reprogramming in obese mice. Toxicol Sci 2013;134:291-303.

90. Al-Eryani L, Wahlang B, Falkner KC, Guardiola JJ, Clair HB, Prough RA, Cave M. Identification of Environmental Chemicals Associated with the Development of Toxicant-associated Fatty Liver Disease in Rodents. Toxicol Pathol 2014.

91. Bligh EG, Dyer WJ. A rapid method of total lipid extraction and purification. Can J Biochem Physiol 1959;37:911-917.

92. Livak KJ, Schmittgen TD. Analysis of relative gene expression data using real-time quantitative PCR and the 2(-Delta Delta $\mathrm{C}(\mathrm{T})$ ) Method. Methods 2001;25:402-408.

93. Lima-Cabello E, Garcia-Mediavilla MV, Miquilena-Colina ME, VargasCastrillon J, Lozano-Rodriguez T, Fernandez-Bermejo M, Olcoz JL, et al. Enhanced expression of pro-inflammatory mediators and liver X-receptorregulated lipogenic genes in non-alcoholic fatty liver disease and hepatitis C. Clin Sci (Lond) 2011;120:239-250.

94. Zhou J, Febbraio M, Wada T, Zhai Y, Kuruba R, He J, Lee JH, et al. Hepatic fatty acid transporter Cd36 is a common target of LXR, PXR, and PPARgamma in promoting steatosis. Gastroenterology 2008;134:556-567. 
95. Lu YC, Yeh WC, Ohashi PS. LPS/TLR4 signal transduction pathway. Cytokine 2008;42:145-151.

96. NTP. (National Toxicology Program). NTP toxicology and carcinogenesis studies of 3,3',4,4',5-pentachlorobiphenyl (PCB 126) (CAS No. 57465-288) in female Harlan Sprague-Dawley rats (Gavage Studies). Natl Toxicol Program Tech Rep Ser 2006:4-246.

97. Al-Salman F, Plant N. Non-coplanar polychlorinated biphenyls (PCBs) are direct agonists for the human pregnane- $\mathrm{X}$ receptor and constitutive androstane receptor, and activate target gene expression in a tissuespecific manner. Toxicol Appl Pharmacol 2012;263:7-13.

98. Robertson LW, Berberian I, Borges T, Chen LC, Chow CK, Glauert HP, Filser JG, et al. Suppression of peroxisomal enzyme activities and cytochrome P450 4A isozyme expression by congeneric polybrominated and polychlorinated biphenyls. PPAR Res 2007;2007:15481.

99. Wahlang B, Falkner KC, Clair HB, Al-Eryani L, Prough RA, States JC, Coslo DM, et al. Human Receptor Activation By Aroclor 1260, a Polychlorinated Biphenyl Mixture. Toxicol Sci 2014.

100. Sugatani J, Wada T, Osabe M, Yamakawa K, Yoshinari K, Miwa M. Dietary inulin alleviates hepatic steatosis and xenobiotics-induced liver injury in rats fed a high-fat and high-sucrose diet: association with the suppression of hepatic cytochrome P450 and hepatocyte nuclear factor 4alpha expression. Drug Metab Dispos 2006;34:1677-1687. 
101. Ghose R, Omoluabi O, Gandhi A, Shah P, Strohacker K, Carpenter KC, McFarlin B, et al. Role of high-fat diet in regulation of gene expression of drug metabolizing enzymes and transporters. Life Sci 2011;89:57-64.

102. Smith AB, Schloemer J, Lowry LK, Smallwood AW, Ligo RN, Tanaka S, Stringer W, et al. Metabolic and health consequences of occupational exposure to polychlorinated biphenyls. $\mathrm{Br} \mathrm{J}$ Ind Med 1982;39:361-369.

103. Chai X, Zeng S, Xie W. Nuclear receptors PXR and CAR: implications for drug metabolism regulation, pharmacogenomics and beyond. Expert Opin Drug Metab Toxicol 2013;9:253-266.

104. Gao J, Xie W. Pregnane X receptor and constitutive androstane receptor at the crossroads of drug metabolism and energy metabolism. Drug Metab Dispos 2010;38:2091-2095.

105. Nakamura K, Moore R, Negishi M, Sueyoshi T. Nuclear pregnane $\mathrm{X}$ receptor cross-talk with FoxA2 to mediate drug-induced regulation of lipid metabolism in fasting mouse liver. J Biol Chem 2007;282:9768-9776.

106. Deng X, Zhang W, I OS, Williams JB, Dong Q, Park EA, Raghow R, et al. FoxO1 inhibits sterol regulatory element-binding protein-1c (SREBP-1c) gene expression via transcription factors Sp1 and SREBP-1c. J Biol Chem 2012;287:20132-20143.

107. Pohjanvirta R, Tuomisto J. Short-term toxicity of 2,3,7,8tetrachlorodibenzo-p-dioxin in laboratory animals: effects, mechanisms, and animal models. Pharmacol Rev 1994;46:483-549. 
108. Kimura A, Naka T, Nakahama T, Chinen I, Masuda K, Nohara K, FujiiKuriyama Y, et al. Aryl hydrocarbon receptor in combination with Stat1 regulates LPS-induced inflammatory responses. J Exp Med 2009;206:2027-2035.

109. Menezes-Garcia Z, Oliveira MC, Lima RL, Soriani FM, Cisalpino D, Botion LM, Teixeira MM, et al. Lack of platelet-activating factor receptor protects mice against diet-induced adipose inflammation and insulin-resistance despite fat pad expansion. Obesity (Silver Spring) 2014;22:663-672.

110. Angrish MM, Mets BD, Jones AD, Zacharewski TR. Dietary fat is a lipid source in 2,3,7,8-tetrachlorodibenzo-rho-dioxin (TCDD)-elicited hepatic steatosis in C57BL/6 mice. Toxicol Sci 2012;128:377-386.

111. Weir JB. New methods for calculating metabolic rate with special reference to protein metabolism. J Physiol 1949;109:1-9.

112. Le Hellard S, Theisen FM, Haberhausen M, Raeder MB, Ferno J, Gebhardt S, Hinney A, et al. Association between the insulin-induced gene 2 (INSIG2) and weight gain in a German sample of antipsychotictreated schizophrenic patients: perturbation of SREBP-controlled lipogenesis in drug-related metabolic adverse effects? Mol Psychiatry 2009;14:308-317.

113. Peterson TR, Sengupta SS, Harris TE, Carmack AE, Kang SA, Balderas E, Guertin DA, et al. mTOR complex 1 regulates lipin 1 localization to control the SREBP pathway. Cell 2011;146:408-420. 
114. Ghosh S, Murinova L, Trnovec T, Loffredo CA, Washington K, Mitra PS, Dutta SK. Biomarkers Linking PCB Exposure and Obesity. Curr Pharm Biotechnol 2014.

115. Gerbal-Chaloin S, Iankova I, Maurel P, Daujat-Chavanieu M. Nuclear receptors in the cross-talk of drug metabolism and inflammation. Drug Metab Rev 2013;45:122-144.

116. Zhou C, Tabb MM, Nelson EL, Grun F, Verma S, Sadatrafiei A, Lin M, et al. Mutual repression between steroid and xenobiotic receptor and NFkappaB signaling pathways links xenobiotic metabolism and inflammation. J Clin Invest 2006;116:2280-2289.

117. Hu G, Xu C, Staudinger JL. Pregnane X receptor is SUMOylated to repress the inflammatory response. J Pharmacol Exp Ther 2010;335:342350.

118. Miao J, Fang S, Bae Y, Kemper JK. Functional inhibitory cross-talk between constitutive androstane receptor and hepatic nuclear factor-4 in hepatic lipid/glucose metabolism is mediated by competition for binding to the DR1 motif and to the common coactivators, GRIP-1 and PGC-1alpha. J Biol Chem 2006;281:14537-14546.

119. Bhalla S, Ozalp C, Fang S, Xiang L, Kemper JK. Ligand-activated pregnane $\mathrm{X}$ receptor interferes with HNF-4 signaling by targeting a common coactivator PGC-1alpha. Functional implications in hepatic cholesterol and glucose metabolism. J Biol Chem 2004;279:45139-45147. 
120. Oda N, Imamura S, Fujita T, Uchida Y, Inagaki K, Kakizawa H, Hayakawa $\mathrm{N}$, et al. The ratio of leptin to adiponectin can be used as an index of insulin resistance. Metabolism 2008;57:268-273.

121. Zhai Y, Wada T, Zhang B, Khadem S, Ren S, Kuruba R, Li S, et al. A functional cross-talk between liver $X$ receptor-alpha and constitutive androstane receptor links lipogenesis and xenobiotic responses. Mol Pharmacol 2010;78:666-674.

122. Gong Y, Lee JN, Lee PC, Goldstein JL, Brown MS, Ye J. Sterol-regulated ubiquitination and degradation of Insig-1 creates a convergent mechanism for feedback control of cholesterol synthesis and uptake. Cell Metab $2006 ; 3: 15-24$.

123. Yellaturu CR, Deng X, Park EA, Raghow R, Elam MB. Insulin enhances the biogenesis of nuclear sterol regulatory element-binding protein (SREBP)-1c by posttranscriptional down-regulation of Insig-2A and its dissociation from SREBP cleavage-activating protein (SCAP).SREBP-1C complex. J Biol Chem 2009;284:31726-31734.

124. Hardie DG, Ashford ML. AMPK: regulating energy balance at the cellular and whole body levels. Physiology (Bethesda) 2014;29:99-107.

125. Haissaguerre M, Saucisse N, Cota D. Influence of mTOR in energy and metabolic homeostasis. Mol Cell Endocrinol 2014.

126. Bakan I, Laplante M. Connecting mTORC1 signaling to SREBP-1 activation. Curr Opin Lipidol 2012;23:226-234. 
127. Yecies JL, Zhang HH, Menon S, Liu S, Yecies D, Lipovsky Al, Gorgun C, et al. Akt stimulates hepatic SREBP1c and lipogenesis through parallel mTORC1-dependent and independent pathways. Cell Metab 2011;14:2132.

128. Kimball SR. Interaction between the AMP-activated protein kinase and mTOR signaling pathways. Med Sci Sports Exerc 2006;38:1958-1964.

129. Yang H, Garzel B, Heyward S, Moeller T, Shapiro P, Wang H. Metformin represses drug-induced expression of CYP2B6 by modulating the constitutive androstane receptor signaling. Mol Pharmacol 2014;85:249260.

130. Zhou J, Zhai Y, Mu Y, Gong H, Uppal H, Toma D, Ren S, et al. A novel pregnane $\mathrm{X}$ receptor-mediated and sterol regulatory element-binding protein-independent lipogenic pathway. J Biol Chem 2006;281:1501315020.

131. Ong KT, Mashek MT, Bu SY, Greenberg AS, Mashek DG. Adipose triglyceride lipase is a major hepatic lipase that regulates triacylglycerol turnover and fatty acid signaling and partitioning. Hepatology 2011;53:116-126.

132. Chakrabarti $P$, Kandror KV. FoxO1 controls insulin-dependent adipose triglyceride lipase (ATGL) expression and lipolysis in adipocytes. J Biol Chem 2009;284:13296-13300. 
133. Chakrabarti P, English T, Karki S, Qiang L, Tao R, Kim J, Luo Z, et al. SIRT1 controls lipolysis in adipocytes via FOXO1-mediated expression of ATGL. J Lipid Res 2011;52:1693-1701.

134. Ueda A, Hamadeh HK, Webb HK, Yamamoto Y, Sueyoshi T, Afshari CA, Lehmann JM, et al. Diverse roles of the nuclear orphan receptor CAR in regulating hepatic genes in response to phenobarbital. Mol Pharmacol 2002;61:1-6.

135. Wolfrum C, Asilmaz E, Luca E, Friedman JM, Stoffel M. Foxa2 regulates lipid metabolism and ketogenesis in the liver during fasting and in diabetes. Nature 2004;432:1027-1032.

136. Louet JF, Hayhurst G, Gonzalez FJ, Girard J, Decaux JF. The coactivator PGC-1 is involved in the regulation of the liver carnitine palmitoyltransferase I gene expression by cAMP in combination with HNF4 alpha and cAMP-response element-binding protein (CREB). J Biol Chem 2002;277:37991-38000.

137. Wolfrum C, Besser D, Luca E, Stoffel M. Insulin regulates the activity of forkhead transcription factor Hnf-3beta/Foxa-2 by Akt-mediated phosphorylation and nuclear/cytosolic localization. Proc Natl Acad Sci U S A 2003;100:11624-11629.

138. Auerbach SS, Dekeyser JG, Stoner MA, Omiecinski CJ. CAR2 displays unique ligand binding and RXRalpha heterodimerization characteristics. Drug Metab Dispos 2007;35:428-439. 
139. Auerbach SS, Stoner MA, Su S, Omiecinski CJ. Retinoid X receptoralpha-dependent transactivation by a naturally occurring structural variant of human constitutive androstane receptor (NR1/3). Mol Pharmacol 2005;68:1239-1253.

140. Omiecinski CJ, Coslo DM, Chen T, Laurenzana EM, Peffer RC. Multispecies analyses of direct activators of the constitutive androstane receptor. Toxicol Sci 2011;123:550-562.

141. Rushneck DR, Beliveau A, Fowler B, Hamilton C, Hoover D, Kaye K, Berg $\mathrm{M}$, et al. Concentrations of dioxin-like PCB congeners in unweathered Aroclors by HRGC/HRMS using EPA Method 1668A. Chemosphere 2004;54:79-87.

142. He J, Gao J, Xu M, Ren S, Stefanovic-Racic M, O'Doherty RM, Xie W. PXR ablation alleviates diet-induced and genetic obesity and insulin resistance in mice. Diabetes 2013.

143. Ducheix S, Podechard N, Lasserre F, Polizzi A, Pommier A, Murzilli S, Di Lisio C, et al. A systems biology approach to the hepatic role of the oxysterol receptor LXR in the regulation of lipogenesis highlights a crosstalk with PPARalpha. Biochimie 2013;95:556-567.

144. Arrebola JP, Pumarega J, Gasull M, Fernandez MF, Martin-Olmedo P, Molina-Molina JM, Fernandez-Rodriguez M, et al. Adipose tissue concentrations of persistent organic pollutants and prevalence of type 2 diabetes in adults from Southern Spain. Environ Res 2013;122:31-37. 
145. Goncharov A, Haase RF, Santiago-Rivera A, Morse G, Akwesasne Task Force on the E, McCaffrey RJ, Rej R, et al. High serum PCBs are associated with elevation of serum lipids and cardiovascular disease in a Native American population. Environ Res 2008;106:226-239.

146. Mayes BA, McConnell EE, Neal BH, Brunner MJ, Hamilton SB, Sullivan TM, Peters AC, et al. Comparative carcinogenicity in Sprague-Dawley rats of the polychlorinated biphenyl mixtures Aroclors 1016, 1242, 1254, and 1260. Toxicol Sci 1998;41:62-76.

147. Parkinson A, Robertson LW, Safe L, Safe S. Polychlorinated biphenyls as inducers of hepatic microsomal enzymes: effects of di-ortho substitution. Chem Biol Interact 1981;35:1-12.

148. Tabb MM, Kholodovych V, Grun F, Zhou C, Welsh WJ, Blumberg B. Highly chlorinated PCBs inhibit the human xenobiotic response mediated by the steroid and xenobiotic receptor (SXR). Environ Health Perspect 2004;112:163-169.

149. Faucette SR, Zhang TC, Moore R, Sueyoshi T, Omiecinski CJ, LeCluyse EL, Negishi $M$, et al. Relative activation of human pregnane $\mathrm{X}$ receptor versus constitutive androstane receptor defines distinct classes of CYP2B6 and CYP3A4 inducers. J Pharmacol Exp Ther 2007;320:72-80.

150. DeKeyser JG, Laurenzana EM, Peterson EC, Chen T, Omiecinski CJ. Selective phthalate activation of naturally occurring human constitutive androstane receptor splice variants and the pregnane $\mathrm{X}$ receptor. Toxicol Sci 2011;120:381-391. 
151. Mutoh S, Sobhany M, Moore R, Perera L, Pedersen L, Sueyoshi T, Negishi M. Phenobarbital Indirectly Activates the Constitutive Active Androstane Receptor (CAR) by Inhibition of Epidermal Growth Factor Receptor Signaling. Sci Signal 2013;6:ra31.

152. Wahlang B, Song M, Beier Jl, Cameron Falkner K, Al-Eryani L, Clair HB, Prough RA, et al. Evaluation of Aroclor 1260 exposure in a mouse model of diet-induced obesity and non-alcoholic fatty liver disease. Toxicol Appl Pharmacol 2014;279:380-390.

153. Clair HB, Falkner KC, Wahlang B, Prough RA, Cave MC. Elevated biomarker-indicated liver disease and pro-inflammatory cytokines are associated with environmental PCB exposure in Anniston, Alabama. Hepatology Special Issue: The 65th Annual Meeting of the American Association for the Study of Liver Diseases: The Liver Meeting 2014;60: 92A-196A.

154. Henquin JC. Triggering and amplifying pathways of regulation of insulin secretion by glucose. Diabetes 2000;49:1751-1760.

155. Zarrouki B, Benterki I, Fontes G, Peyot ML, Seda O, Prentki M, Poitout V. Epidermal growth factor receptor signaling promotes pancreatic beta-cell proliferation in response to nutrient excess in rats through mTOR and FOXM1. Diabetes 2014;63:982-993.

156. Hakonen E, Ustinov J, Palgi J, Miettinen PJ, Otonkoski T. EGFR signaling promotes beta-cell proliferation and survivin expression during pregnancy. PLoS One 2014;9:e93651. 
157. Niknam Y, Feng W, Cherednichenko G, Dong Y, Joshi SN, Vyas SM, Lehmler $\mathrm{HJ}$, et al. Structure-activity relationship of selected meta- and para-hydroxylated non-dioxin like polychlorinated biphenyls: from single RyR1 channels to muscle dysfunction. Toxicol Sci 2013;136:500-513.

158. Fritsch EB, Pessah IN. Structure-activity relationship of non-coplanar polychlorinated biphenyls toward skeletal muscle ryanodine receptors in rainbow trout (Oncorhynchus mykiss). Aquat Toxicol 2013;140-141:204212.

159. Lesmana R, Shimokawa N, Takatsuru Y, Iwasaki T, Koibuchi N. Lactational exposure to hydroxylated polychlorinated biphenyl (OH-PCB 106) causes hyperactivity in male rat pups by aberrant increase in dopamine and its receptor. Environ Toxicol 2014;29:876-883.

160. Fernandes EC, Hendriks HS, van Kleef RG, Reniers A, Andersson PL, van den Berg M, Westerink RH. Activation and potentiation of human GABAA receptors by non-dioxin-like PCBs depends on chlorination pattern. Toxicol Sci 2010;118:183-190.

161. Angrish MM, Jones AD, Harkema JR, Zacharewski TR. Aryl hydrocarbon receptor-mediated induction of Stearoyl-CoA desaturase 1 alters hepatic fatty acid composition in TCDD-elicited steatosis. Toxicol Sci 2011;124:299-310. 


\section{APPENDIX}

\section{ELSEVIER LICENSE \\ TERMS AND CONDITIONS}

Oct 06, 2014

This is a License Agreement between Banrida Wahlang ("You") and Elsevier ("Elsevier") provided by Copyright Clearance Center ("CCC"). The license consists of your order details, the terms and conditions provided by Elsevier, and the payment terms and conditions.

\section{All payments must be made in full to CCC. For payment instructions,} please see information listed at the bottom of this form.

Supplier

Elsevier Limited

The Boulevard,Langford Lane

Kidlington,Oxford,OX5 1GB,UK

Registered Company Number

1982084

Customer name

Banrida Wahlang

Customer address

505 S Hancock Street CTRB 542C

LOUISVILLE, KY 40202

License number

3483230586886

License date

Oct 06, 2014

Licensed content publisher

Elsevier

Licensed content publication

Toxicology and Applied Pharmacology

Licensed content title

Evaluation of Aroclor 1260 exposure in a mouse model of dietinduced

obesity and non-alcoholic fatty liver disease

Licensed content author

Banrida Wahlang,Ming Song,Juliane I. Beier,K. Cameron Falkner, Laila Al

Eryani,Heather B. Clair,Russell A. Prough,Tanasa S. Osborne,David E. Malarkey,J. Christopher States,Matthew C. Cave

Licensed content date

15 September 2014 
Licensed content volume number

279

Licensed content issue number

3

Number of pages

11

Start Page

380

End Page

390

Type of Use

reuse in a thesis/dissertation

Intended publisher of new work

other

Portion

full article

Format

both print and electronic

Are you the author of this Elsevier article?

Yes

Will you be translating?

No

Order reference number

Dissertation2

Title of your thesis/dissertation

EVALUATING THE EFFECTS OF AROCLOR 1260 IN NON-ALCOHOLIC

FATTY LIVER DISEASE: ROLE OF XENOBIOTIC RECEPTORS

Expected completion date

Dec 2014

Estimated size (number of pages)

150

Elsevier VAT number

GB 494627212

Permissions price

0.00 USD

VAT/Local Sales Tax

0.00 USD / $0.00 \mathrm{GBP}$

Total

0.00 USD

Terms and Conditions

INTRODUCTION

1. The publisher for this copyrighted material is Elsevier. By clicking "accept" in connection with completing this licensing transaction, you agree that the following terms and conditions apply to this transaction (along with the Billing and Payment terms and conditions established by Copyright Clearance Center, Inc. ("CCC"), at 
the time that you opened your Rightslink account and that are available at any time at http://myaccount.copyright.com).

\section{GENERAL TERMS}

2. Elsevier hereby grants you permission to reproduce the aforementioned material subject to the terms and conditions indicated.

3. Acknowledgement: If any part of the material to be used (for example, figures) has appeared in our publication with credit or acknowledgement to another source, permission must also be sought from that source. If such permission is not obtained then that material may not be included in your publication/copies. Suitable acknowledgement to the source must be made, either as a footnote or in a reference list at the end of your publication, as follows:

"Reprinted from Publication title, Vol /edition number, Author(s), Title of article / title of chapter, Pages No., Copyright (Year), with permission from Elsevier [OR APPLICABLE SOCIETY COPYRIGHT OWNER]." Also Lancet special credit "Reprinted from The Lancet, Vol. number, Author(s), Title of article, Pages No., Copyright (Year), with permission from Elsevier."

4. Reproduction of this material is confined to the purpose and/or media for which permission is hereby given.

5. Altering/Modifying Material: Not Permitted. However figures and illustrations may be altered/adapted minimally to serve your work. Any other abbreviations, additions, deletions and/or any other alterations shall be made only with prior written authorization of Elsevier Ltd. (Please contact Elsevier at permissions@elsevier.com)

6. If the permission fee for the requested use of our material is waived in this instance, please be advised that your future requests for Elsevier materials may attract a fee.

7. Reservation of Rights: Publisher reserves all rights not specifically granted in the combination of (i) the license details provided by you and accepted in the course of this licensing transaction, (ii) these terms and conditions and (iii) CCC's Billing and Payment terms and conditions.

8. License Contingent Upon Payment: While you may exercise the rights licensed immediately upon issuance of the license at the end of the licensing process for the transaction, provided that you have disclosed complete and accurate details of your proposed use, no license is finally effective unless and until full payment is received from you (either by publisher or by CCC) as provided in CCC's Billing and Payment terms and conditions. If full payment is not received on a timely basis, then any license preliminarily granted shall be deemed automatically revoked and shall be void as if never granted. Further, in the event that you breach any of these terms and conditions or any of CCC's Billing and Payment terms and conditions, the license is automatically revoked and shall be void as if never granted. Use of materials as described in a revoked license, as well as any use of the materials beyond the scope of an unrevoked license, may constitute copyright infringement and publisher reserves the right to take any and all action to protect its copyright in the materials.

9. Warranties: Publisher makes no representations or warranties with respect to the licensed material. 
10. Indemnity: You hereby indemnify and agree to hold harmless publisher and $\mathrm{CCC}$, and their respective officers, directors, employees and agents, from and against any and all claims arising out of your use of the licensed material other than as specifically authorized pursuant to this license.

11. No Transfer of License: This license is personal to you and may not be sublicensed, assigned, or transferred by you to any other person without publisher's written permission.

12. No Amendment Except in Writing: This license may not be amended except in a writing signed by both parties (or, in the case of publisher, by CCC on publisher's behalf).

13. Objection to Contrary Terms: Publisher hereby objects to any terms contained in any purchase order, acknowledgment, check endorsement or other writing prepared by you, which terms are inconsistent with these terms and conditions or CCC's Billing and Payment terms and conditions. These terms and conditions, together with CCC's Billing and Payment terms and conditions (which are incorporated herein), comprise the entire agreement between you and publisher (and CCC) concerning this licensing transaction. In the event of any conflict between your obligations established by these terms and conditions and those established by CCC's Billing and Payment terms and conditions, these terms and conditions shall control.

14. Revocation: Elsevier or Copyright Clearance Center may deny the permissions described in this License at their sole discretion, for any reason or no reason, with a full refund payable to you. Notice of such denial will be made using the contact information provided by you.

Failure to receive such notice will not alter or invalidate the denial. In no event will Elsevier or Copyright Clearance Center be responsible or liable for any costs, expenses or damage incurred by you as a result of a denial of your permission request, other than a refund of the amount(s) paid by you to Elsevier and/or Copyright Clearance Center for denied permissions.

\section{LIMITED LICENSE}

The following terms and conditions apply only to specific license types:

15. Translation: This permission is granted for non-exclusive world English rights only unless your license was granted for translation rights. If you licensed translation rights you may only translate this content into the languages you requested. A professional translator must perform all translations and reproduce the content word for word preserving the integrity of the article. If this license is to re-use 1 or 2 figures then permission is granted for non-exclusive world rights in all languages.

16. Posting licensed content on any Website: The following terms and conditions apply as follows: Licensing material from an Elsevier journal: All content posted to the web site must maintain the copyright information line on the bottom of each image; A hyper-text must be included to the Homepage of the journal from which you are licensing at http://www.sciencedirect.com/science/journal/xxxxx or the Elsevier homepage for books at http://www.elsevier.com; Central Storage: This license does not include 
permission for a scanned version of the material to be stored in a central repository such as that provided by

Heron/XanEdu.

Licensing material from an Elsevier book: A hyper-text link must be included to the Elsevier homepage at http://www.elsevier.com . All content posted to the web site must maintain the copyright information line on the bottom of each image. Posting licensed content on Electronic reserve: In addition to the above the following clauses are applicable: The web site must be password-protected and made available only to bona fide students registered on a relevant course. This permission is granted for 1 year only. You may obtain a new license for future website posting.

For journal authors: the following clauses are applicable in addition to the above:

Permission granted is limited to the author accepted manuscript version* of your paper.

${ }^{*}$ Accepted Author Manuscript (AAM) Definition: An accepted author manuscript (AAM) is the author's version of the manuscript of an article that has been accepted for publication and which may include any author-incorporated changes suggested through the processes of submission processing, peer review, and editor-author communications. AAMs do not include other publisher value-added contributions such as copy-editing, formatting, technical enhancements and (if relevant) pagination. You are not allowed to download and post the published journal article (whether PDF or HTML, proof or final version), nor may you scan the printed edition to create an electronic version. A hyper-text must be included to the Homepage of the journal from which you are licensing at http://www.sciencedirect.com/science/journal/xxxxx. As part of our normal production process, you will receive an e-mail notice when your article appears on Elsevier's online service ScienceDirect (www.sciencedirect.com). That e-mail will include the article's Digital Object Identifier (DOI). This number provides the electronic link to the published article and should be included in the posting of your personal version. We ask that you wait until you receive this e-mail and have the DOI to do any posting.

Posting to a repository: Authors may post their AAM immediately to their employer's institutional repository for internal use only and may make their manuscript publically available after the journal-specific embargo period has ended. Please also refer to Elsevier's Article Posting Policy for further information.

18. For book authors the following clauses are applicable in addition to the above: Authors are permitted to place a brief summary of their work online only.. You are not allowed to download and post the published electronic version of your chapter, nor may you scan the printed edition to create an electronic version.

19. Posting to a repository: Authors are permitted to post a summary of their chapter only in their institution's repository.

20. Thesis/Dissertation: If your license is for use in a thesis/dissertation your thesis may be submitted to your institution in either print or electronic form. 
Should your thesis be published commercially, please reapply for permission. These requirements include permission for the Library and Archives of Canada to supply single copies, on demand, of the complete thesis and include permission for UMI to supply single copies, on demand, of the complete thesis. Should your thesis be published commercially, please reapply for permission.

\section{Elsevier Open Access Terms and Conditions}

Elsevier publishes Open Access articles in both its Open Access journals and via its Open Access articles option in subscription journals. Authors publishing in an Open Access journal or who choose to make their article Open Access in an Elsevier subscription journal select one of the following Creative Commons user licenses, which define how a reader may reuse their work: Creative Commons Attribution License (CC BY), Creative Commons Attribution - Non Commercial ShareAlike (CC BY NC SA) and Creative Commons Attribution - Non Commercial - No Derivatives (CC BY NC ND) Terms \& Conditions applicable to all Elsevier Open Access articles: Any reuse of the article must not represent the author as endorsing the adaptation of the article nor should the article be modified in such a way as to damage the author's honour or reputation. The author(s) must be appropriately credited. If any part of the material to be used (for example, figures) has appeared in our publication with credit or acknowledgement to another source it is the responsibility of the user to ensure their reuse complies with the terms and conditions determined by the rights holder.

\section{Additional Terms \& Conditions applicable to each Creative Commons user} license:

CC BY: You may distribute and copy the article, create extracts, abstracts, and other revised versions, adaptations or derivative works of or from an article (such as a translation), to include in a collective work (such as an anthology), to text or data mine the article, including for commercial purposes without permission from Elsevier

CC BY NC SA: For non-commercial purposes you may distribute and copy the article, create extracts, abstracts and other revised versions, adaptations or derivative works of or from an article (such as a translation), to include in a collective work (such as an anthology), to text and data mine the article and license new adaptations or creations under identical terms without permission from Elsevier

CC BY NC ND: For non-commercial purposes you may distribute and copy the article and include it in a collective work (such as an anthology), provided you do not alter or modify the article, without permission from Elsevier

Any commercial reuse of Open Access articles published with a CC BY NC SA or CC BY

NC ND license requires permission from Elsevier and will be subject to a fee. Commercial reuse includes:

Promotional purposes (advertising or marketing)

Commercial exploitation ( e.g. a product for sale or loan)

Systematic distribution (for a fee or free of charge)

Please refer to Elsevier's Open Access Policy for further information. 
21. Other Conditions: v1.6 Questions? customercare@copyright.com or +1855-239-3415 (toll free in the US) or +1-978-646-2777. Gratis licenses (referencing $\$ 0$ in the Total field) are free. Please retain this printable license for your reference. No payment is required.

\section{OXFORD UNIVERSITY PRESS LICENSE}

\section{TERMS AND CONDITIONS}

Oct 06, 2014

This is a License Agreement between Banrida Wahlang ("You") and Oxford University Press ("Oxford University Press") provided by Copyright Clearance Center ("CCC"). The license consists of your order details, the terms and conditions provided by Oxford University Press, and the payment terms and conditions.

All payments must be made in full to CCC. For payment instructions, please see information listed at the bottom of this form.

License Number

3483230135496

License date

Oct 06, 2014

Licensed content publisher

Oxford University Press

Licensed content publication

Toxicological Sciences

Licensed content title

Human Receptor Activation by Aroclor 1260, a Polychlorinated Biphenyl Mixture Licensed content author

Banrida Wahlang, K. Cameron Falkner, Heather B. Clair, Laila Al- Eryani, Russell

A. Prough, J. Christopher States, Denise M. Coslo, Curtis J. Omiecinski, Matthew

C. Cave

Licensed content date

August 2014

Type of Use

Thesis/Dissertation

Institution name

None

Title of your work

EVALUATING THE EFFECTS OF AROCLOR 1260 IN NON-ALCOHOLIC

FATTY LIVER DISEASE: ROLE OF XENOBIOTIC RECEPTORS

Publisher of your work

n/a

Expected publication date

Dec 2014

Permissions cost

0.00 USD

Value added tax 
0.00 USD

Total

0.00 USD

Total

0.00 USD

Terms and Conditions

\section{STANDARD TERMS AND CONDITIONS FOR REPRODUCTION OF} MATERIAL FROM AN OXFORD UNIVERSITY PRESS JOURNAL

1. Use of the material is restricted to the type of use specified in your order details.

2. This permission covers the use of the material in the English language in the following territory: world. If you have requested additional permission to translate this material, the terms and conditions of this reuse will be set out in clause 12 . 3. This permission is limited to the particular use authorized in (1) above and does not allow you to sanction its use elsewhere in any other format other than specified above, nor does it apply to quotations, images, artistic works etc that have been reproduced from other sources which may be part of the material to be used.

4. No alteration, omission or addition is made to the material without our written consent. Permission must be re-cleared with Oxford University Press if/when you decide to reprint.

5. The following credit line appears wherever the material is used: author, title, journal, year, volume, issue number, pagination, by permission of Oxford University Press or the sponsoring society if the journal is a society journal. Where a journal is being published on behalf of a learned society, the details of that society must be included in the credit line.

6 . For the reproduction of a full article from an Oxford University Press journal for whatever purpose, the corresponding author of the material concerned should be informed of the proposed use. Contact details for the corresponding authors of all Oxford University Press journal contact can be found alongside either the abstract or full text of the article concerned, accessible from www.oxfordjournals.org Should there be a problem clearing these rights, please contact journals.permissions@oup.com

7. If the credit line or acknowledgement in our publication indicates that any of the figures, images or photos was reproduced, drawn or modified from an earlier source it will be necessary for you to clear this permission with the original publisher as well. If this permission has not been obtained, please note that this material cannot be included in your publication/photocopies.

8. While you may exercise the rights licensed immediately upon issuance of the license at the end of the licensing process for the transaction, provided that you have disclosed complete and accurate details of your proposed use, no license is finally effective unless and until full payment is received from you (either by Oxford University Press or by Copyright Clearance Center (CCC)) as provided in CCC's Billing and Payment terms and conditions. If full payment is not received on a timely basis, then any license preliminarily granted shall be deemed automatically revoked and shall be void as if never granted. Further, in the event 
that you breach any of these terms and conditions or any of CCC's Billing and Payment terms and conditions, the license is automatically revoked and shall be void as if never granted. Use of materials as described in a revoked license, as well as any use of the materials beyond the scope of an unrevoked license, may constitute copyright infringement and Oxford University Press reserves the right to take any and all action to protect its copyright in the materials.

9. This license is personal to you and may not be sublicensed, assigned or transferred by you to any other person without Oxford University Press's written permission.

10. Oxford University Press reserves all rights not specifically granted in the combination of (i) the license details provided by you and accepted in the course of this licensing transaction, (ii) these terms and conditions and (iii) CCC's Billing and Payment terms and conditions.

11. You hereby indemnify and agree to hold harmless Oxford University Press and $\mathrm{CCC}$, and their respective officers, directors, employs and agents, from and against any and all claims arising out of your use of the licensed material other than as specifically authorized pursuant to this license.

12. Other Terms and Conditions: v1.4 Questions?

customercare@copyright.com or +1-855-239-3415 (toll free in the US) or +1-978-646-2777. Gratis licenses (referencing $\$ 0$ in the Total field) are free. Please retain this printable license for your reference. No payment is required. 


\section{CURRICULUM VITAE \\ BANRIDA WAHLANG}

\section{Email: b0wah101@louisville.edu}

Phone: 502-718-0357

\section{EDUCATION}

2012-present Ph.D. candidate (Pharmacology \& Toxicology), University of Louisville, Louisville, K.Y. U.S.A.

2012, May M.S. (Pharmacology \& Toxicology), University of Louisville, Louisville, K.Y. U.S.A. Thesis title: Polychlorinated biphenyl 153 exacerbates non-alcoholic fatty liver disease in C57/BI6 mice.

2010, June

M.S. (Pharmaceutics), National Institute of Pharmaceutical Education and Research (NIPER), S.A.S. Nagar, India.

Thesis title: Identification of permeability related hurdles in the oral delivery of curcumin, using Caco-2 cell model.

2008, June Bachelors (Pharmacy), University of Delhi, New Delhi, India. 


\section{PEER-REVIEWED PUBLICATIONS}

1. Evaluation of Aroclor 1260 exposure in a mouse model of diet-induced obesity and non-alcoholic fatty liver disease. Wahlang B, Song M, Beier JI, Cameron Falkner K, Al-Eryani L, Clair HB, Prough RA, Osborne TS, Malarkey DE, Christopher States J, Cave MC. Toxicol Appl Pharmacol. 2014, 279(3), 380-390.

2. Identification of environmental chemicals associated with the development of toxicant associated fatty liver disease in rodents. Al-Eryani L, Wahlang B, Falkner KC, Guardiola JJ, Clair HB, Prough RA, Cave MC. Toxicol Pathol. 2014. pii: 0192623314549960.

3. Human Receptor Activation by Aroclor 1260, a Polychlorinated Biphenyl Mixture. Wahlang B, Falkner KC, Clair HB, Al-Eryani L, Prough RA, States JC, Coslo DM, Omiecinski CJ, Cave MC. Tox Sci. 2014, 140(2), 283-97.

4. Polychlorinated biphenyl 153 is a diet-dependent obesogen that worsens nonalcoholic fatty liver disease in male C57BL6/J mice. Wahlang B, Falkner KC, Gregory B, Ansert D, Young D, Conklin DJ, Bhatnagar A, McClain CJ, Cave M. J Nutr Biochem. 2013, 24(9), 1587-95.

5. Toxicant-associated Steatohepatitis. Wahlang B, Beier JI, Clair HB, Bellis-Jones HJ, Falkner KC, McClain CJ, Cave MC. Toxicol Pathol. 2013, 41(2):343-60. 
6. Effect of Counterions on Physicochemical Properties of Prazosin Salts. Kumar L, Meena CL, Pawar YB, Wahlang B, Tikoo K, Jain R, Bansal AK. AAPS PharmSciTech. 2013, 14(1):141-50.

7. Metabolomic Analysis of the Effects of Polychlorinated Biphenyls in Nonalcoholic Fatty Liver Disease. Shi X, Wahlang B, Wei X, Yin X, Falkner KC, Prough RA, Kim SH, Mueller EG, McClain CJ, Cave M, Zhang X.J Proteome Res. 2012, 11(7):3805-15.

8. Mechanistic insights into PEPT1-mediated transport of a novel antiepileptic, NP-647. Khomane KS, Nandekar PP, Wahlang B, Bagul P, Shaikh N, Pawar YB, Meena CL, Sangamwar AT, Jain R, Tikoo K, Bansal AK. Mol Pharm. 2012, 9(9):2458-68.

9. Contribution of formulation and excipients towards enhanced permeation of curcumin. Wahlang B, Kabra D, Pawar YB, Tikoo K, Bansal AK. Arzneimittelforschung. 2012, 62(2):88-93.

10. Identification of permeability-related hurdles in oral delivery of curcumin using the Caco-2 cell model. Wahlang B, Pawar YB, Bansal AK. Eur J Pharm Biopharm. 2011, 77(2):275-82.

\section{PRESENTATIONS: NATIONAL/LOCAL MEETINGS}

2014 Poster presentation, American Association for the Study of Liver Diseases (AASLD), Boston, MA, U.S.A.

2014 Oral Presentation, Ohio Valley Society of Toxicology (OVSOT), Annual Meeting, Dayton, OH, U.S.A 
2014 Poster presentation, Research! Louisville, Louisville, Louisville, KY, U.S.A.

2014 Poster presentation, NIEHS Tamburro Symposium on Environmental Chemicals and Liver Disease, Louisville, KY, U.S.A.

2014 Poster presentation, Great Lakes Drug Metabolism and Disposition Meeting, Indianapolis, IN, U.S.A.

2014 Poster presentation, Society of Toxicology (SOT), Phoenix, AZ, U.S.A.

2013 Poster presentation, AASLD, Washington D.C., U.S.A.

2013 Poster presentation, Research! Louisville, Louisville, Louisville, KY, U.S.A.

2013 Oral Presentation, OVSOT, Annual Meeting, Louisville, KY. U.S.A.

2013 Poster Presentation, OVSOT, Annual Meeting, Louisville, K.Y. U.S.A.

2013 Oral Presentation, OVSOT Summer Student Meeting, Mason, $\mathrm{OH}$, U.S.A.

2013 Poster presentation, SOT, San Antonio, TX, U.S.A.

2012 Poster presentation, Research! Louisville, Louisville, Louisville, KY, U.S.A.

2011 Poster presentation, Research! Louisville, Louisville, Louisville, KY, U.S.A.

2011 Poster presentation, OVSOT, Dayton, OH, U.S.A.

2010 Poster presentation, Drug Metabolism and Pharmacokinetics 
(DMPK) Symposium, S.A.S. Nagar, Mohali, India.

2009 Poster presentation, Indian Pharmaceutical Congress 2009, Ahmedabad, India.

\section{ABSTRACTS}

1. Evaluating the role of nuclear receptors in environmental liver disease. B.

Wahlang, K.C. Falkner, M. Song, H. Clair, R. A. Prough, and M. Cave. NIEHS Tamburro Symposium on Environmental Chemicals and Liver Disease 2014 and Research! Louisville, 2014.

2. Do PCBs activate Constitutive Androstane Receptor through a non-ligand binding mechanism? A. T. Orr, R. A. Prough, K. C. Falkner, B. Wahlang, and M. Cave. Research! Louisville, 2014.

3. The protective role of CAR and PXR in Aroclor 1260-induced Steatohepatitis. B. Wahlang, K.C. Falkner, M. Song, H. Clair, R. A. Prough, and M. Cave. AASLD, 2014.

4. Elevated Biomarker-indicated Liver Disease and Pro-inflammatory Cytokines are Associated with Environmental PCB Exposure in Anniston, Alabama. H. Clair, K.C. Falkner, B. Wahlang, R. A. Prough and M. Cave. AASLD, 2014.

5. PCB Regulation of Hepatic Nuclear Receptors: Implications for Hepatic Steatosis. R.A. Prough, B. Wahlang, K.C. Falkner, H.B. Clair, L.A. AlEryani, J. C. States, C.J. Omiecinski, and M.C. Cave. Presentation S6, 5th 
Asia Pacific International Society for the Study of Xenobiotics. Tianjin, China, 2014.

6. The Role of Nuclear Receptors in Aroclor 1260-induced Steatohepatitis.

B. Wahlang, K.C. Falkner, M. Song, H. Clair, R. Prough, and M. Cave. Great Lakes Drug Metabolism and Disposition Meeting, 2014.

7. FXR Activation Is Suppressed By STARD5 in Hkc-8 Human Renal Proximal Tubule Cells. B.J. Clark, S.J. Khundmiri, E.D. Lederer, M. Cave and B. Wahlang. The Endocrine Society, 2014.

8. Aroclor 1260 Exposure Causes Steatohepatitis and Activates Hepatic Receptors in an Animal Model of Diet-Induced Obesity. B. Wahlang, M. Song, J. Beier, L. Al-Eryani, H.B. Clair, J.J. Guardiola, K.C. Falkner, R.A. Prough, J.C. States and M. Cave. SOT, 2014.

9. Identification of Environmental Chemicals which could contribute to Nonalcoholic Fatty Liver Disease by Nuclear Receptor Activation. L. AlEryani, B. Wahlang, K.C. Falkner, H.B. Clair, R.A. Prough, J.C. States and M. Cave. AASLD, 2013 and SOT, 2014.

10. Database Mining for Pregnane Xenobiotic Receptor (PXR) Ligands Using Toxcast Database and PXR Activation by Organochlorine Pesticides. L. Al-Eryani, B. Wahlang, H.B. Clair, J. J. Guardiola, K.C. Falkner, R.A. Prough and M. Cave. AASLD, 2013.

11. Aroclor 1260 exposure worsens hepatic and systemic inflammation in an animal model of diet-induced obesity and non-alcoholic fatty liver disease. B. Wahlang, K.C. Falkner, H.B. Clair, L. Al-Eryani, J. Christopher States, 
R.A. Prough, and M. Cave. OVSOT Annual Meeting, 2013 and AASLD, 2013.

12. Hepatic receptor activation by polychlorinated biphenyls - implications for xenobiotic/energy metabolism and nonalcoholic fatty liver disease. B.

Wahlang, K.C. Falkner, H.B. Clair, L. Al-Eryani, J. Christopher States, R.A. Prough, and M. Cave. OVSOT Summer Student Meeting, 2013 and AASLD 2013.

13. Human transcription factor activation by polychlorinated biphenyls and organochlorine pesticides. B. Wahlang, Laila Al-Eryani, K.C. Falkner, H.J. Bellis-Jones, H.B. Clair, R.A. Prough, and M. Cave. Society of Toxicology (SOT), 2013.

14. PCB 153 alters hepatic lipid metabolism and the hepatic metabolome in C57BL/6 mice fed a high fat diet: Nutrient-toxin interactions in NAFLD. B.

Wahlang, X. Shi, X. Zhang, K.C. Falkner, C.J. McClain, R. Prough, M. Cave. AASLD, 2012.

15. LXR and PXR crosstalk on DR4 response elements is sequence dependent. K.C. Falkner, B. Wahlang, H.J. Bellis-Jones, H.B. Clair, R.A. Prough, and M. Cave. International Society for the Study of Xenobiotics (ISSX), 2012.

16. Aroclor 1260 is a direct agonist of the human Pregnane and Xenobiotic Receptor. H.B. Clair, B. Wahlang, K.C. Falkner, R.A. Prough, and M. Cave. Research! Louisville, 2012. 
17. Aroclor 1260 does not appear to be a direct agonist or antagonist of the Liver X-Receptor $\alpha$. H.J. Bellis-Jones, B. Wahlang, K.C. Falkner, R.A. Prough, and M. Cave. Research! Louisville, 2012.

18. Liver X Receptor and Pregnane Xenobiotic Receptor crosstalk on Direct Repeat 4 (DR4) response elements is sequence dependent. B. Wahlang, K.C. Falkner, H.J. Bellis-Jones, H.B. Clair, R.A. Prough, and M. Cave. Research! Louisville, 2012.

19. The metabolic effects of PCB 153 depend on nutrient interactions in obesity/nonalcoholic fatty liver disease. B. Wahlang, X. Shi, X. Zhang, K.C. Falkner, C.J. McClain, R. Prough, M. Cave. Digestive Disease Week (DDW), 2012.

20. Polychlorinated Biphenyl 153 worsens hepatic steatosis in mice fed a high fat diet and differentially regulates 40 liver metabolites: preliminary results of a metabolomics analysis. B. Wahlang, X. Zhang, K. C. Falkner, C. J. McClain, M. Cave. American Association for the Study of Liver Diseases (AASLD), 2011.

21. A comparison of serum adipocytokines, cytokeratin 18, and antioxidants in human subjects with steatohepatitis due to alcohol (ASH), obesity (NASH), and industrial chemicals (TASH). H. Shah, B. Wheeler, Y. Mannery, K.C. Falkner, B. Wahlang, C.J. McClain, M. Cave. Research! Louisville, 2011. 
22. Polychlorinated Biphenyl 153 worsens non-alcoholic fatty liver disease in C57BL/6 mice. B. Wahlang, K.C. Falkner, D.J. Conklin, C.J. McClain, M. Cave. OVSOT, 2011 and SOT, 2012.

23. Intestinal transport mechanism of a novel thyrotropin releasing hormone. K Khomane, B. Wahlang, Y.B. Pawar, C.L. Meena, R. Jain, A.K. Bansal. DMPK, 2011.

24. Determination of curcumin's permeability using the Caco-2 cell model. B. Wahlang, Y.B. Pawar, A.K. Bansal. DMPK, 2011.

25. HPLC method for determination of curcumin for its application in Caco-2 permeability assays. B. Wahlang, Y.B. Pawar, A.K. Bansal. IPC, 2009.

26. The Caco-2 Cell Model: A Useful Tool in Drug Discovery and Development. B. Wahlang, Y.B. Pawar, S.R. Patil and A.K. Bansal. CRIPS 2009, 10(2), 29-34. (Apr-Jun).

\section{PROFESSIONAL MEMBERSHIPS}

2014-present Risk Assessment Specialty Section

2013-present American Society for Pharmacology \& Experimental

Therapeutics.

2013-present Molecular and Systems Biology Specialty Section

2013-present Ohio Valley SOT Regional Chapter

2013-present Occupational and Public Health Specialty Section

2013-present Society of Toxicology.

2012-present Kentucky Academy of Science. 
$2009-2010$ American Association of Pharmaceutical Scientists (AAPS) NIPER Student Chapter.

\section{PROFESSIONAL TRAINING AND DEVELOPMENT}

2014 Completed CEd-Tox course "Basic Principles in Human Risk Assessment"

2013 Grant Writing Academy, University of Louisville.

2009 Online general course on Intellectual Property Rights, WIPO, Switzerland.

\section{LEADERSHIP}

2014 Member, Communications Subcommittee, GSLC, SOT.

2014 Member, Graduate Student Leadership Committee, SOT.

2014 Student Representative, Ohio Valley SOT Student Chapter.

2012 Treasurer, American International Relations Club (AIRC), University of Louisville.

2011 Public Relations Officer, AIRC, University of Louisville.

2009 Secretary, Pharmaceutics Club, NIPER.

\section{AWARDS AND HONORS}

2014

Graduate Dean's Citation

2014

$2^{\text {nd }}$ Place, PhD Platform Presentation, OVSOT Annual

Meeting 
2014 \& 2012 Sponsored Research Tuition Award, School of

Interdisciplinary and Graduate Studies, University of

Louisville.

2014 \& 2013 Travel Award, Graduate School Council, University of

Louisville.

2013

Best PhD Platform Presentation, OVSOT Annual Meeting.

2013

Travel Award, School of Medicine Research Committee, University of Louisville.

2011 Finalist, Research! Louisville Graduate Poster Session.

2010-2012 Integrated Programs in Biomedical Sciences Fellowship

2009 Runner up, Business Plan, 81MG, NIPER.

2008 All India $119^{\text {th }}$ Rank, General Aptitude Test in

Engineering, IIS Bangalore, India.

\section{SERVICE: ACADEMIC AND COMMUNITY-RELATED}

2014

2014

2014

2014

2013-present
Volunteer/Attendee, Midwest Membrane Trafficking \& Signaling Symposium, Louisville.

Proctor, Patient SIMS on Autonomics, School of Medicine, University of Louisville.

Organizer, OVSOT Annual Summer Student Meeting Judge, Manual High School Science Fair. Member, Golden Key International Honor Society, 
University of Louisville.

2013-summer Volunteer, Children's Hospital Foundation, Kosair

Children's Hospital, Louisville, K.Y.

2012-present Member, University of Louisville Study Abroad.

2011-present Member, Indian Students Association, University of

Louisville.

2008-2006 Member, Indian Pharmaceutical Graduate Association (IPGA). 